\title{
IMISCOE
}

RESEARCH

\section{Framing Immigrant Integration}

Dutch Research-Policy Dialogues in Comparative Perspective

PETER SCHOLTEN

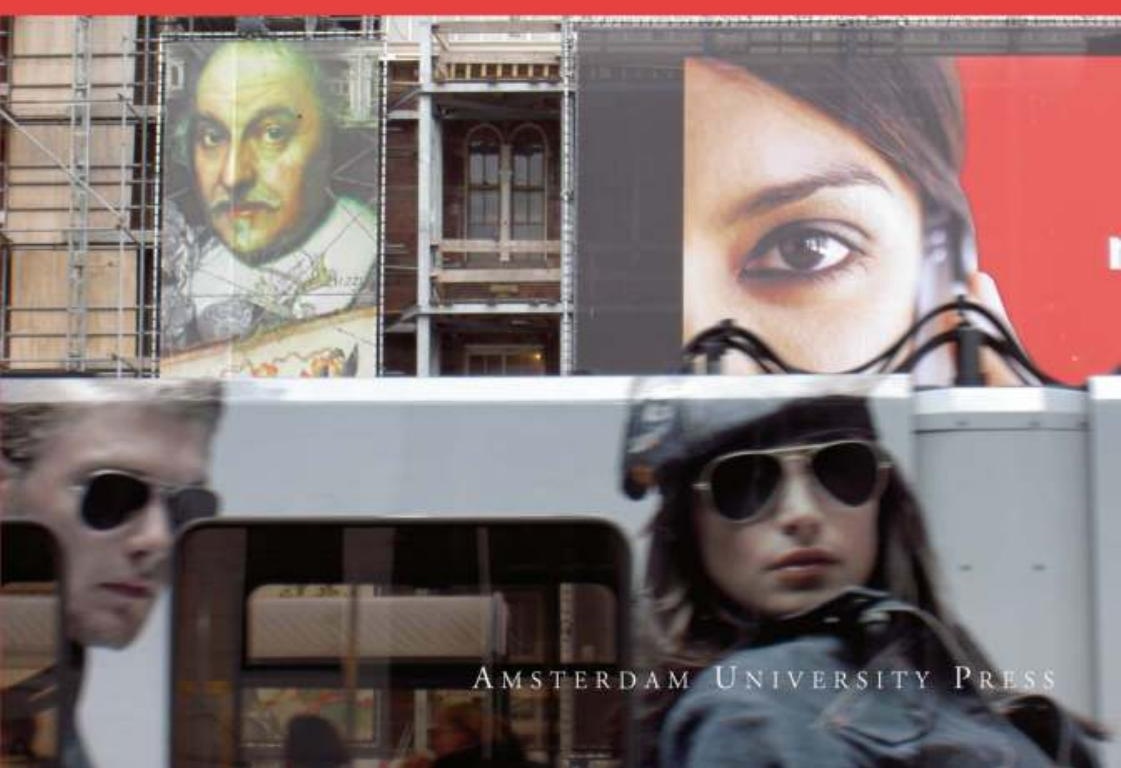


Framing Immigrant Integration 


\section{IMISCOE}

\section{International Migration, Integration and Social Cohesion in Europe}

The IMISCOE Research Network unites researchers from, at present, 28 institutes specialising in studies of international migration, integration and social cohesion in Europe. What began in 2004 as a Network of Excellence sponsored by the Sixth Framework Programme of the European Commission has become, as of April 2009, an independent self-funding endeavour open to qualified researchers and research institutes worldwide. From the start, IMISCOE has promoted integrated, multidisciplinary and globally comparative research led by scholars from various branches of the economic and social sciences, the humanities and law. The Network furthers existing studies and pioneers new scholarship on migration and migrant integration. Encouraging innovative lines of inquiry key to European policymaking and governance is also a priority.

The IMISCOE-Amsterdam University Press Series makes the Network's findings and results available to researchers, policymakers and practitioners, the media and other interested stakeholders. High-quality manuscripts authored by Network members and cooperating partners are evaluated by external peer reviews and the IMISCOE Editorial Committee. The Committee comprises the following members:

Tiziana Caponio, Department of Political Studies, University of Turin / Forum for International and European Research on Immigration (FIERI), Turin, Italy

Michael Collyer, Sussex Centre for Migration Research (SCMR), University of Sussex, United Kingdom

Rosita Fibbi, Swiss Forum for Migration and Population Studies (SFM), University of Neuchâtel / Institute of Social Sciences, University of Lausanne, Switzerland

Agata Górny, Centre of Migration Research (CMR) / Faculty of Economic Sciences, University of Warsaw, Poland

Albert Kraler, International Centre for Migration Policy Development (ICMPD), Vienna, Austria

Leo Lucassen, Institute of History, Leiden University, The Netherlands

Jorge Malheiros, Centre of Geographical Studies (CEG), University of Lisbon, Portugal

Marco Martiniello, National Fund for Scientific Research (FNRS), Brussels / Center for Ethnic and Migration Studies (CEDEM), University of Liège, Belgium

Patrick Simon, National Demographic Institute (INED), Paris, France

Miri Song, School of Social Policy and Sociology, University of Kent, United Kingdom

More information and how to join the Network can be found at www.imiscoe.org. 


\title{
Framing Immigrant Integration
}

\author{
Dutch Research-Policy Dialogues in \\ Comparative Perspective
}

\author{
Peter Scholten
}

IMISCOE Research

AMSTERdaM UNIVERSity PRESS 


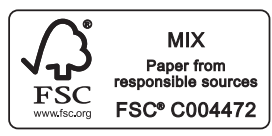

Cover design: Studio Jan de Boer BNO, Amsterdam Cover photo: Jeroen Doomernik (www.jeroendoomernik.net) Layout: The DocWorkers, Almere

$\begin{array}{ll}\text { ISBN } & 9789089642844 \\ \text { e-ISBN } & 9789048513604 \\ \text { NUR } & 741 / 763\end{array}$

(C) Peter Scholten / Amsterdam University Press, Amsterdam 2011

All rights reserved. Without limiting the rights under copyright reserved above, no part of this book may be reproduced, stored in or introduced into a retrieval system, or transmitted, in any form or by any means (electronic, mechanical, photocopying, recording or otherwise) without the written permission of both the copyright owner and the author of the book. 


\section{Contents}

$\begin{array}{ll}\text { Preface } & 7\end{array}$

$\begin{array}{ll}\text { Abbreviations } & 11\end{array}$

1 Introduction $\quad 13$

1.1 Immigrant integration: An intractable social problem 16

1.2 The co-evolution of immigrant integration research and policies in the Netherlands 19

1.3 Research-policy dialogues on immigrant integration 23

1.4 Dutch exceptionalism? 25

2 Research-policy dialogues and the framing

of immigrant integration $\quad 29$

2.1 Structuralist-constructivism: Beyond 'models thinking' and radical constructivism $\quad 30$

2.2 Framing immigrant integration $\quad 35$

2.3 The research-policy nexus $\quad 42$

2.4 Research-policy dialogues and critical frame reflection 51

2.5 Research design $\quad 58$

2.6 Conclusion $\quad 65$

3 Frames and frameshifts in Dutch immigrant integration policy $\begin{array}{ll}\text { and research } & 67\end{array}$

3.1 The Dutch multicultural model 68

3.2 The rise and fall of policy frames 69

3.3 Frames in immigrant integration research 79

3.4 Conclusion $\quad 85$

4 Technocracy and the construction of the Dutch multicultural model (1978-1983) 87

4.1 The rise of a multiculturalist model 87

4.2 The construction of a technocratic nexus 101

4.3 Technocracy and the rise of the multicultural model 121

4.4 Conclusion 132 
$5 \quad$ Enlightenment and the rise of universalism (1989-1994) 135

5.1 The turn towards universalism 136

5.2 The construction of enlightenment 153

$\begin{array}{lll}5.3 & \text { Enlightenment and frameshifts } & 173\end{array}$

$\begin{array}{ll}5.4 \text { Conclusions } & 180\end{array}$

6 The engineering of the assimilationist turn (2000-2004) 183

6.1 The turn towards assimilationism and transnationalism 184

6.2 The research-policy nexus on the line 198

6.3 Engineering and the selective co-production of research and policy 214

$\begin{array}{ll}6.4 \text { Conclusion } & 225\end{array}$

7 Dutch exceptionalism? Immigrant integration research and policies in France, Germany and the United Kingdom 229

$\begin{array}{lll}7.1 \text { France } & 230\end{array}$

$\begin{array}{lll}7.2 & \text { Germany } & 246\end{array}$

$\begin{array}{lll}7.3 & \text { The UK } & 259\end{array}$

$\begin{array}{ll}7.4 \text { Conclusions: Dutch exceptionalism? } & 271\end{array}$

8 Conclusion: Towards reflective $\begin{array}{ll}\text { research-policy dialogues? } & 277\end{array}$

8.1 Beyond the Dutch multicultural model 278

8.2 The Dutch research-policy nexus on the line 281

8.3 Dutch exceptionalism? 284

8.4 Towards reflexive research-policy dialogues? 287

$\begin{array}{ll}\text { Notes } & 289\end{array}$

$\begin{array}{ll}\text { References } & 301\end{array}$ 


\section{Preface}

This book is the outcome of the many years that I have journeyed in the field of migration research and policymaking. Being a policy scientist, I entered this field as a relative outsider. The thorny issue of immigrant integration challenged many of the lessons I had been taught as a student of public policy. It defied much of what I had learnt about rationally defining social problems, organising processes of policymaking and evaluating policy programmes. Somehow, much of the traditional policy scientist's toolkit did not make sense when confronted with the 'wicked policy problem'. This became even more evident when, just after the turn of the millennium in the midst of my schooling in public policy and public administration, immigrant integration emerged forcefully onto the Dutch public and political agenda. In spite of decades of policy, the Dutch approach was now being declared a 'failure'. Furthermore, the credibility of those experts who had been closely involved in the making of these policies was now publicly on the line. Clearly, the role of scientific experts in the making of Dutch immigrant integration policies was changing.

This is precisely what motivated me to undertake research on the relations between migration research and policymaking in the Netherlands. Culminating in my dissertation entitled 'Constructing immigrant policies: Research-policy relations and immigrant integration policymaking in the Netherlands', I completed my PhD in January 2008 at the University of Twente in Enschede, the Netherlands. The entire process was an adventure into the very lively and dynamic field of Dutch migration research. After immersing myself in migration literature and policy documents, I talked to many key scholars and policymakers and visited the most relevant institutes in the field. This lifted the lid on a complex, dynamic and, at times, contested area. Making sense of this controversial domain that I had ventured into required me to review the many traditional ideas I held about the role of research in policymaking. As a policy scientist, this has shaped my understanding of how policymaking works in practice and how the division of labour between research and policy is actually produced and reproduced.

Since obtaining my $\mathrm{PhD}$, I have continued working on the reconceptualisation of research-policy relations in this specific policy domain. This led to an international conference in May 2008 entitled Research-Policy 
Dialogues on Migration and Integration in Europe, which I organised together with Rinus Penninx, who has been involved in such research-policy dialogues for decades, both in the Netherlands and at a wider European level. The gathering was graciously hosted by the University of Twente's Department of Social Risks and Safety Studies, where I held an assistant professorship. It was supported by the IMISCOE Research Network concerned with, as its acronym suggests, international migration, integration and social cohesion in Europe and by the Institute for Governance Studies (IGS). The conference created an opportunity for policymakers and migration scholars to engage in dialogue on their mutual relations. It also allowed me to broaden the scope of my interest in research-policy dialogues beyond the Netherlands, posing questions such as whether or not it is an exceptional case, or if there is something more general that we can learn from the Dutch experience?

This book is the product of my broader reflections on the dialogue between migration and migration policymaking. With it, I intend to contribute to a better empirical and theoretical understanding of research-policy dialogues on intractable policy controversies such as immigrant integration. In this respect, I consider immigrant integration a revelatory case as it represents a policy domain in which traditional ideas about policymaking and the role of social research within it have been seriously challenged. Furthermore, I intend to contribute to shaping more reflective research-policy dialogues in this field in the future. Clearly, both governments and researchers are struggling to come to terms with the 'wicked' problem, yet there appears to be little progress in terms of taming the ongoing controversies on immigrant integration. This study may not necessarily provide a resolution for the problem of immigrant integration, but it does bring about more reflexivity in the related dialogues.

This study would not have been possible without the attention and help of various people, whom I cannot thank enough for getting me acquainted with the world of migration research. Rinus Penninx has not only been an invaluable source of information, but also a great motivator throughout my research; without him there would have been no Research-Policy Dialogues conference and no book about it either. The Department of Social Risks and Safety Studies honed my skills as a researcher and, in particular, my promoters Bert de Vroom and Romke van der Veen dealt patiently with my uncertainties and shortcomings as I developed as a social scientist. Han Entzinger, one of the supervisors of my Master's thesis, was a tremendous inspiration and instructor from early on, and he has continued to be there for me, meticulously reviewing various drafts of this book. Christina Boswell and Virginie Guiraudon were of enormous assistance in broadening the scope of my research interest beyond the Dutch case and reviewing parts of the international comparative chapter. Rob Hoppe has been my guide in the world of science studies and helped me develop my 
PhD dissertation into this book. Let me thank Leo Lucassen from Leiden University and Elke Winter from the University of Ottawa for their rigorous reviews and constructive comments on its latest versions. I also wish to thank Anna Yeadell and Karina Hof for their unwearied reviewing of the final text.

Finally, much gratitude is due to my young family, who had to live with this evolving manuscript for at least four summers. My wife Amal and our children Safae and Ilyas have been a huge support, remaining endlessly patient and indulgent. It is to them, therefore, that I dedicate this book.

Rotterdam, April 2011 



\section{Abbreviations}

\begin{tabular}{|c|c|c|}
\hline Abbreviation & Name in full & English name \\
\hline ACOM & $\begin{array}{l}\text { Adviescommissie Onderzoek } \\
\text { Minderheden }\end{array}$ & $\begin{array}{l}\text { Advisory Committee on Minorities } \\
\text { Research }\end{array}$ \\
\hline BAMF & $\begin{array}{l}\text { Bundesamtes für Migration und } \\
\text { Flüchtlinge }\end{array}$ & $\begin{array}{l}\text { German Federal Office for Migration } \\
\text { and Refugees }\end{array}$ \\
\hline CBS & Centraal Bureau voor Statistiek & National Statistics Office \\
\hline CAZ & Commissariat van Ambonezenzorg & $\begin{array}{l}\text { Commissioner's Office for Welfare of } \\
\text { Moluccans }\end{array}$ \\
\hline CDA & Christen-Democratisch Appel & Christian Democratic Appeal Party \\
\hline CRE & & Commission for Racial Equality \\
\hline CRER & & $\begin{array}{l}\text { Centre for Race and Equality } \\
\text { Research }\end{array}$ \\
\hline D66 & Democraten 1966 & Liberal Democratic Party \\
\hline DCIM & $\begin{array}{l}\text { Directie Coördinatie Integratie } \\
\text { Minderheden }\end{array}$ & $\begin{array}{l}\text { Directorate for the Coordination of } \\
\text { the Minorities }\end{array}$ \\
\hline EBB & Enquête Beroepsbevolking & Labour Force Surveys \\
\hline FAS & $\begin{array}{l}\text { Fond d'Action Sociale pour les } \\
\text { Travailleurs Immigrés et Leur } \\
\text { Familles }\end{array}$ & $\begin{array}{l}\text { Social Work Fund for Labor } \\
\text { Migrants and Their Families }\end{array}$ \\
\hline GELD & $\begin{array}{l}\text { Group d'Études et de Lutte contre } \\
\text { les Discriminations }\end{array}$ & $\begin{array}{l}\text { Group for Studying and Combating } \\
\text { Discrimination }\end{array}$ \\
\hline HALDE & $\begin{array}{l}\text { Haute Autorité de Lutte contre les } \\
\text { Discriminations et pour l'Egalité }\end{array}$ & $\begin{array}{l}\text { The French Equal Opportunities and } \\
\text { Anti-Discrimination Commission }\end{array}$ \\
\hline ICM & & $\begin{array}{l}\text { Independent Commission on } \\
\text { Migration to Germany }\end{array}$ \\
\hline INED & $\begin{array}{l}\text { Institut National des Études } \\
\text { Démographiques }\end{array}$ & $\begin{array}{l}\text { National Institute for Demographic } \\
\text { Studies }\end{array}$ \\
\hline INSEE & $\begin{array}{l}\text { Institut National de la Statistique et } \\
\text { des Études Économiques }\end{array}$ & French Statistics Office \\
\hline IRR & & $\begin{array}{l}\text { Institute for Race Relations } \\
\text { Research }\end{array}$ \\
\hline ISEO & $\begin{array}{l}\text { Instituut voor Sociologisch- } \\
\text { Economisch Onderzoek }\end{array}$ & $\begin{array}{l}\text { Institute for Social and Economic } \\
\text { Research }\end{array}$ \\
\hline IWM & Inspraakorgaan Welzijn Molukkers & $\begin{array}{l}\text { Consultation Body for the Welfare of } \\
\text { Moluccans }\end{array}$ \\
\hline LAO & $\begin{array}{l}\text { Landelijk Advies en Overleg Orgaan } \\
\text { voor Minderheden }\end{array}$ & $\begin{array}{l}\text { National Advisory and Consultation } \\
\text { Structure for Minorities }\end{array}$ \\
\hline LISW & $\begin{array}{l}\text { Landelijk Inspraakorgaan van } \\
\text { Surinaamse Welzijnsinstellingen }\end{array}$ & $\begin{array}{l}\text { Foundation of Surinamese Welfare } \\
\text { Organisations }\end{array}$ \\
\hline LPF & Lijst Pim Fortuyn & Pim Fortuyn Party \\
\hline
\end{tabular}




\begin{tabular}{lll}
\hline Abbreviation & Name in full & English name \\
\hline Ministry of CRM & Ministerie van Cultuur, Recreatie en & Ministry of Culture, Recreation and \\
& Maatschappelijk Werk & Social Work \\
NCB & Nederlands Centrum Buitenlanders & Dutch Centre for Foreigners \\
OVB & Onderzoek en Verificatie Bureau & Research and Verification Office \\
PSI & & Policy Science Institute \\
PvdA & Partij van de Arbeid & Social Democrat Party \\
REMPLOD & Project voor de Reintegratie van & Reintegration of Emigrant Manpower \\
& Migranten en Bevordering van & and Promotion of Local \\
& Locale Kansen en Ontwikkeling & Opportunities and Development \\
& & Project \\
RMO & Raad voor Maatschappelijke & Council for Social Development \\
& Ontwikkeling & \\
ROB & Raad voor het Openbaar Bestuur & Council for Public Government \\
SCP & Sociaal en Cultureel Planbureau & Social and Cultural Planning Office \\
& & (also Institute for Social Sciences) \\
TWCM & Tijdelijke Wetenschappelijke & Temporary Scientific Committee for \\
VVD & Commissie Minderhedenbeleid & Minorities Policy \\
VWJ & Volkspartij voor Vrijheid en & Liberal Party \\
WODC & Democratie & \\
& Verwey-Jonker Instituut & Verwey-Jonker Institute \\
WRR & Wetenschappelijk Onderzoek-en & Scientific Research and \\
& Documentatiecentrum & Documentation Centre of the \\
& & Department of Justice \\
& Regeringsbeleid & Scientific Council for Government \\
& & Policy \\
\hline
\end{tabular}




\section{Introduction}

Dutch society has long been held up as an exemplary case of successful multiculturalism. Yet, the 'multicultural model' is now widely - and sometimes wildly - rejected by large parts of the population. The controversies about this model have become a symbol of how contemporary governments struggle to tame complex, heated issues such as immigrant integration and, more generally, how they cope with rapid societal transformation as the result of seemingly unstoppable phenomena like globalisation, migration and cultural diversification. The incorporation of migrants has evolved into a major social and political concern for contemporary Dutch society. Now that the modernist belief in rational societal steering has significantly decayed, how can governments respond to the challenges of our time?

Since the late 1970s, when governments first developed policy efforts aimed at immigrant integration, there has been an explosion of social science research in this area. Initially, a relatively small network of researchers - mainly anthropologists and sociologists - focused on the social and cultural position of migrants or 'ethnic minorities' in Dutch society. In particular, in the late 1970s and early 1980s, the Advisory Committee on Minorities Research (ACOM) played a key role in stimulating and coordinating research on this issue. At that time, ACOM held a rather exclusive position in this field. However, since the late 1980s, the research network has broadened extensively, with a variety of social science disciplines becoming involved, as well as an increasing institutional fragmentation of the research field. Today, immigrant integration research takes place at almost all universities in the Netherlands, and a variety of specialised institutes has evolved, for instance, in the production of quantitative data on the integration process, studying integration processes at the local level and providing science-based policy advice.

Social science research has, at various stages, played a major part in the development of Dutch immigrant integration policy. Particularly in its infancy, ACOM's research had a direct relation with the science-based policy recommendations from the Scientific Council for Government Policy (Wetenschappelijke Raad voor het Regeringsbeleid, WRR) and development of the Dutch ethnic minorities policy of the 1980s and the integration policy of the 1990s. Social researchers in this period played a central role 
in the production of the multicultural model for which the Netherlands has become so well known.

However, the participation of social researchers and research institutes in policy development has become fiercely contested. Whereas the researchpolicy nexus had been one of the key axes for policy development in the 1980s, during the 1990s the nexus was gradually dismantled and by 1992 ACOM was discontinued. WRR, whose reports were the main precursors of policy change from the late 1970 s to the 1980 s, issued two reports in 2001 and 2007 that remained largely ignored. Furthermore, the credibility of social researchers was publicly put on the line. The association between researchers and the Dutch multicultural model became particularly controversial once this model was publicly rejected. At the same time, political developments after the turn of the millennium, including the rise of Pim Fortuyn and other populist politicians, such as Geert Wilders, contributed to immigrant integration becoming highly politicised. Rather than grounding their policies in scientific recommendations, the politicians' objective was to show that they had a distinct political vision that took the concerns of 'ordinary people' very seriously. Finally, the emerging political cynicism towards scientific expertise seems to have been fuelled further by manifesting conflicts of knowledge among researchers. The consensus that once underpinned the multicultural model now made way for several competing discourses, which contributed to growing uncertainty about which knowledge claims to select.

A recent episode vividly illustrates how research-policy relations in this domain became contested. In 2003, following what was dubbed the 'long year of 2002' in Dutch politics - characterised by the rise and subsequent assassination of Fortuyn - Dutch Parliament established an investigative committee to enquire why the country's integration policies showed little signs of success. This committee commissioned an extensive study by a well-known research institute to evaluate the effects of past policies. However, the study concluded that the integration process had been relatively successful in some aspects. In the fields of education and labour participation, the committee recorded significant progress - this was seen as indication of the successful integration of immigrants into Dutch society.

Researchers apparently understood integration in terms of the participation of immigrants in these domains. Yet, this definition of integration was not broadly shared in government or politics. Disagreement emerged over what immigrant integration actually meant. The researchers and the parliamentary committee were highly criticised in public and political debates. Leading politicians discarded the conclusions of the researchers as naïve and biased, and held on to their original conclusion that the policy was a failure. Government referred to crucial areas such as social cohesion, religion and criminality, which the investigative committee had ignored. The government could agree that the policy was partially successful, but also 
insisted that it was unsuccessful in the aforementioned areas. Clearly, instead of providing a new impetus for immigrant integration policy, this research and the parliamentary investigative committee added yet another episode to the ongoing controversies surrounding the issue.

This episode illustrates the difficulties that researchers and policymakers face when the very definition of an underlying problem is contested. Researchers, politicians and policymakers involved in this episode focused on different facets of immigrant integration, and had differing ideas about how the integration process should be evaluated. Their various understandings led them to select different truth claims and interpret available evidence differently so as to come to an evaluation of either policy success or failure.

Furthermore, the dialogues put the division of labour between research and policy at stake. The credibility of the concerned researchers and research institutes was called into question because of their alleged multicultural bias and their involvement in developing the policies that they were now supposed to evaluate. In addition, the committee's decision to ask researchers to evaluate the policy received scathing criticism, as it was considered the task of government to provide a new policy approach, not that of researchers. The year 2002 was a vexing one in Dutch politics. It led to wide rejection of what was considered an elitist way of policymaking, reflecting a perceived interference by scientific expertise and systematic politicisation. The disagreement in this episode was not only about the definition and understanding of immigrant integration, it was also about how research-policy dialogues should be organised in this social process of problem definition.

This book aims to unravel how and why changes in the research-policy nexus were connected to changing definitions of immigrant integration in policy and research. It does not seek to explain how and why these changes in definitions took place, but rather to discuss the role the research-policy nexus has played in these changes. From a sociological and policy science perspective, it aims to explain that nexus by analysing its changing make-up over the past decades, as well as by analysing how and why its consequently varying shapes influenced the definition of immigrant integration in policy and research. Explored, too, will be the extent to which the patterns of research-policy relations in the Dutch case are unique, or whether they represent a broader prototype that can also be found in other European countries - namely, France, Germany and the United Kingdom. In other words, is there a Dutch exceptionalism? Or are there more general patterns in how the research-policy nexus contributes to the rise or fall of specific problem definitions?

This book also aims to contribute to a better empirical and theoretical understanding of research-policy relations in the field of immigrant integration. Although it is commonly recognised that research is a driving factor 
in how immigrant integration policies are instituted across Europe, the research-policy nexus has been - surprisingly - overlooked as an object of empirical examination. There are a few notable exceptions, such as Boswell (2009) and Favell (2001) who emphasise the importance of the research-policy nexus for the development of immigrant integration research. Favell even speaks of a strong correlation between the development of integration policies and what he describes as an 'integration paradigm' in immigrant integration research. To develop a better understanding of the past development of immigrant integration research and to further develop this subject as an autonomous research field, it is crucial to understand how their nexus affects both policies and research.

In theoretical terms, the book borrows insights from policy sciences, sociology and science studies. A typology of different forms of researchpolicy nexus (enlightenment, engineering, bureaucracy, technocracy) will be applied to interpret and compare research-policy relations in different periods and across countries. The endeavour here is to contribute to a better understanding of how research-policy dialogues can be organised in such a way that 'dialogues of the deaf' (Van Eeten 1999) - where frame differences inhibit constructive dialogues, as in the immigrant integration scenario described above - can be averted. Further, under what conditions can the research-policy relations contribute to critical dialogues between research and policy at the level of problem definition?

\subsection{Immigrant integration: An intractable social problem}

The Netherlands has become known worldwide for its multicultural approach to immigrant integration. In both national and international literature, there is a prevailing description of the Dutch approach in terms of a national 'multicultural model'. This model is characterised by a tendency to institutionalise cultural pluralism in the belief that the cultural emancipation of immigrant minorities is the key to their integration into Dutch society. It is frequently connected to the Netherlands' history of pillarisation, which yielded an institutional differentiation of large sections of society into different national minorities (Catholics, Protestants, socialists, liberals).

Upon closer inspection, it becomes apparent that several discourses or 'models' of integration have coexisted and competed in the Netherlands over the past decades. Alongside multiculturalism, there has also been a more social-economic discourse, which stresses participation in areas such as education and labour, as well as a more cultural assimilationist discourse, which emphasises the importance of national identity, norms and values and social cohesion in relation to immigrant integration (Entzinger 2005). In fact, the idea of cultural assimilation has become more prominent over the past decade, as Dutch policy experienced the same 'assimilationist 
turn' observed in many other European countries in this period (Joppke \& Morawska 2003).

It seems, then, that rather than one dominant multicultural model's presence, it is a persistent uncertainty regarding how to model the approach to immigrant integration that characterises Dutch policymaking in recent decades. Indeed, a multiplicity of models marks the struggle to come to terms with this intractable social problem. Dutch society has struggled with various facets of immigrant integration for some time. This includes the arrival and position of migrants in society, as well as the wider effects on society itself. When migrants started to arrive in the Netherlands following World War II, the Dutch had a tradition of spreading themselves across the globe rather than being faced with migration at home. There were relatively early experiences with immigration, such as the influx of Protestants (Huguenots) from France. However, from the second half of the twentieth century onwards - roughly parallel to decolonisation - the Netherlands met a growing scale of migration.

Various categories of migrants can be traced. Firstly, colonial migrants arriving from Surinam, the Dutch Antilles and the Moluccas. This group also included so-called repatriates from the former Dutch East Indies (Schuster 1999). Secondly, in the 1960s, labour migrants began arriving from, in particular, the Mediterranean countries (Spain, Italy, Yugoslavia, Greece, Morocco, Tunisia and Turkey). Thirdly, family migrants could be distinguished, incorporating both the reunion and formation of families by migrants who had already settled in the Netherlands. Finally, especially from the 1990s onwards, refugee migrants have come to the Netherlands from Africa, the Middle East, Eastern Europe and the Far East.

At the beginning of the new millennium, migration's consequences were becoming increasingly obvious in Dutch society. In 2005, the Netherlands was home to 3.1 million immigrants (defined as people born outside the Netherlands, or those with at least one parent born outside the country). This amounted to 19.2 per cent of the Dutch population. ${ }^{1}$ For the same year in the major cities of Amsterdam and Rotterdam, immigrants comprised as much as 34.2 per cent and 35.1 per cent, respectively, of the municipal population. ${ }^{2}$ The largest immigrant groups in the Netherlands, as defined by national origin, are Turkish $(320,000)$, Surinamese $(309,000)$ and Moroccans $(272,800) .^{3}$ In addition to the country's traditional migrant groups - including Moluccans, Southern Europeans, Chinese, Antilleans and Arubans - new migrant groups have arrived, including Iraqis, Iranians, Pakistanis, Afghans and Syrians. An indication of the Netherlands' flourishing cultural and religious diversity is found in the numbers - in 2004, Muslims in Dutch society reached a total of 944,000 , or 5.8 per cent of the Dutch population. ${ }^{4}$ Only recently did this immigration trend break, notably due to the rise in emigration figures. ${ }^{5}$ 
In spite of this migration history, it has often proven difficult to define the consequences of migration for Dutch society and, in turn, to develop appropriate strategies for coping with these consequences. Although immigrant integration is commonly defined as a social problem, its meaning has remained unclear, uncertain and even contested. Some commentators speak of emancipation or 'integration with retention of identity', while others refer to adaptation, participation or segregation. In fact, the notion of integration has been subject to controversy in academic literature as well as in political debates because of its presumed normative bias. In addition, policy approaches to immigrant integration by various countries have diverged strongly over the past decades. Whereas the French have adopted an assimilative approach, the Germans have stressed social-economic participation and the British have followed their own national form of multiculturalism.

It can appear that the only given in immigrant integration is the migrants themselves. However, the definition of what a migrant is has also proven to be a complex and, at times, controversial issue. Migrants can be divided into various categories, as mentioned above, and also into national or ethnic groups or communities (e.g. Turkish, Surinamese, Moroccan). They can also be placed into one broad category of individuals (non-natives or, as called in Dutch, 'allochtonen' ${ }^{, 6}$. Any method for defining 'migrants' leads to questions about why some groups or categories are included while others are not. For instance, Chinese migrants and migrants from Western European countries who are resident in the Netherlands are not defined as minorities that need to be 'integrated'. Furthermore, a distinction is often made between first-, second- and even third-generation migrants, depending on whether an individual or one parent or grandparent is born outside the Netherlands. Moreover, there has been controversy over whether migrants must be defined at all. More and more migrants are becoming naturalised as Dutch national citizens, while sometimes also maintaining their original nationality. Attempts to specify those migrants who need to be integrated (versus those who do not) has incited criticism about the labelling effect this process has on them and its adverse effect on their integration (Rath 1991).

Even if migrants are defined in general terms, there is no general theory of how immigrant integration is to be achieved. The position of migrants is multifaceted. A distinction is often made in the literature between the social-economic, social-cultural and political-legal position of migrants (Fermin 1997: 19). This concerns social-economic issues such as educational achievements, labour market participation and housing; social-cultural issues such as cultural organisations, discrimination, racism and social cohesion; and political-legal issues such as naturalisation regulations, dual nationality, equal treatment regulations and voting rights. As the investigative committee from 2003 illustrated, different actors will deem differing facets of the position of migrants as being most central to integration. For 
instance, in spite of the progress observed in social-economic domains such as education and labour, other members of the committee held on to the conclusion that integration had failed because of insufficient progress in, primarily, the social-cultural domain.

Finally, how immigrant integration is defined draws on many broader societal values. Immigrant integration is a value-laden notion that has often been connected to specific normative conceptions of the nation-state. In fact, it is the nation-state that defines international migration and that defines immigrant integration as a social issue. For many countries, the definition of a migrant and consequent approaches to immigrant integration are correlated with nation-state conceptions (such as foreigners in the exclusionary ethnic German state, racial minorities in multiracial British society and mere immigrants in the inclusive French Republic). In the Netherlands, too, immigrant integration has become associated with nationbuilding legacies such as the history of pillarism and tolerance for religious and cultural differences. Moreover, at the turn of the millennium, immigrant integration became an important issue for the Netherlands' revision of its national imagined community in the context of globalisation as an ongoing social process.

Thus, immigrant integration is a far from self-evident notion. Although 'integration' has become broadly accepted in academic and policy discourse in the Netherlands, as in many other European countries (Favell 2001: 3), its meaning has been weakly articulated. Rather, as the Dutch case will reveal, there is a multiplicity of models - or 'frames', as I will describe them - that provide a specific meaning to integration. This multiplicity marks immigrant integration as a so-called 'wicked problem' (Rittel \& Webber 1973) or an 'intractable controversy' (Rein \& Schön 1994). These are problems that seemingly defy definition and resolution and which involve a multiplicity of problem definitions, also referred to as 'frames'. Moreover, they are seemingly resistant to resolution through studying the underlying facts, as the facts are themselves often selected and interpreted very differently.

\subsection{The co-evolution of immigrant integration research and policies in the Netherlands}

The multiplicity of problem frames that characterises these intractable controversies is reflected in immigrant integration policy and research in the Netherlands. Both have struggled over the past decades to come to terms with this complex social problem. Rather than there being one dominant national model of integration, as is often suggested in national and international migration literature, previous decades have witnessed the rise and fall of several models of integration. 
In fact, neither research nor policy spoke of immigrant 'integration' until the 1990s. Before then, terminology referred to emancipation, the eventual return of temporary migrants or 'international commuters'. Since the 1990 s, the meaning of integration has remained contested, as the example of the investigative committee discussed above illustrates. In addition, migrants have been defined inconsistently over the years - as guest labourers, as ethnic or cultural minorities, as allochthonous or as newcomers and 'oldcomers'. Immigrant integration has also endured various explanations, for instance, in terms of structural impediments to the emancipation of particular groups or citizenship on the part of migrants themselves. It has been categorised in different normative perspectives, such as cultural equality in a multicultural society, social-economic equity in a viable welfare state and national social-cultural cohesion in an age of globalisation.

These diverging interpretations have contributed to a series of shifts in Dutch immigrant integration policies in recent decades (Entzinger 2005). The development of this policy area has followed a rifted pattern at times (Scholten \& Timmermans 2004). Until about the 1970s, only ad hoc welfare measures existed for temporary migrants. In the 1980 s there was a minorities policy, the 1990s saw an integration policy and, since 2003, there has been a shift towards an integration policy 'new style'. Throughout these policy episodes, immigrant integration was defined in different and sometimes conflicting ways (Snel \& Scholten 2005; VWJ 2004). For instance, policy in the 1970 s was aimed at preventing integration so as to facilitate return migration. This contrasts with later policies endeavouring to promote integration. Furthermore, the minorities policy of the 1980s provided various facilities to groups, as opposed to the subsequent integration policy, which instead focused on individual migrants.

Changes in terms of how immigrant integration has been defined have also occurred in immigrant integration research. In the 1970s and especially the 1980s, there was a dominant minorities paradigm (Bovenkerk 1984; Rath 1991). This paradigm has since been challenged by other ways of understanding immigrant integration that have evolved since the 1990s. Later research invoked the citizenship or integration paradigm, as well as perspectives seeing immigrant integration as linked to processes of internationalisation and globalisation (Entzinger 2002; WRR 2001b; Van Amersfoort 2001) or to rising concerns about national identity and social cohesion (Koopmans 2003; SCP 2003). The disagreements on how to define and understand immigrant integration show that research on the issue has been a far from coherent enterprise. Rather, it has been subject to controversies about what integration means, how it should be studied and what the role of research about integration should be.

The aim of this book is to analyse empirically the dialogues between research and policy in the construction of these frames of immigrant integration, as well as to contribute to theory-building on how these mutual 
dialogues affect developments in both policy and immigrant integration research. There are important indications that the research-policy nexus has been pivotal in shaping policies and research in the Netherlands over the past decades. The literature on immigrant integration policymaking contains many references to the prominent role that research institutes, advisory bodies and particular experts have played in this domain (Entzinger 1984, 2003; Penninx 1988b, 2005). For instance, several reports by the Scientific Council for Government Policy (WRR) have had a major impact on policy turning points in recent years (De Jong 2002; VWJ 2004). Various other institutes on the research-policy nexus, including ACOM and the Social and Cultural Planning Office (SCP) have also had an important influence on policy developments. The status of the research-policy nexus as a venue for policy development has also invoked harsh criticism. It has been argued, for instance, that scientific expertise has interfered with ethnic expertise (Penninx 1988b: 27; Van Putten 1990: 361). Scientific expertise has also been said to have facilitated the depoliticisation of this issue, offering an alternative venue for policymaking that allowed avoiding open political debates (De Beus 1998; Rath 2001; Van Amersfoort 1984).

Furthermore, the research-policy nexus influenced the development of specific problem definitions in scientific research. For instance, government research programming and the establishment of ACOM for the coordination of research contributed to the development of a minorities paradigm that defined immigrants as ethnic minorities characterised by socialeconomic deprivation and social-cultural deviance (Rath 1991). In addition, government-associated institutes, such as SCP, coordinated their selection and acquisition of scientific data on the position of migrants according to government demands for information. After the turn of the millennium, public and political discourse put more emphasis on social-cultural issues. In response, SCP (2002: 13) started to pay more attention to social-cultural integration. Researchers and research institutes were often strongly oriented towards, or associated with, national government institutes (Favell 2001: 10). Critics have argued that the policy involvement of research in this area contributed to the rise of specific problem definitions and the exclusion of alternatives (Rath 2001: 140). Moreover, the alleged 'symbiosis' (Van Amersfoort 1984) between research and national government institutes contributed to a highly national orientation in terms of research on immigrant integration. Only during the last ten years, as a result of the researchpolicy nexus rising on local and European levels (Geddes 2005), has the national orientation been challenged by more international or post-national perspectives.

The research-policy nexus's indelible contribution to shaping problem understandings in research and policy is indicated by a number of obvious parallels during the periods in which each domain underwent change. At the end of the 1970s and in the early 1980s, both research and policy came 
to view immigrant integration in terms of social-cultural emancipation and social-cultural participation of ethnic minorities (the aforementioned minorities paradigm and minorities policy). Later, at the end of the 1980s and especially in the early 1990s, the problem framing in both fields changed towards a more individualist orientation regarding citizenship and socialeconomic participation (i.e. the citizenship paradigm and integration policy). Finally, after the turn of the millennium, policy and research went through another period of significant change, though this time not entirely in the direction of a shared understanding on immigrant integration (i.e. transnationalism, assimilationism and the integration policy 'new style'). This suggests that immigrant integration research and policy have, at least to some extent, co-evolved in terms of the ways in which they define and understand immigrant integration (Timmermans \& Scholten 2006).

There was, however, no single given or fixed research-policy nexus. Different actors participated during different periods, including ACOM, WRR, SCP, the Department of Culture, Recreation and Social Work, the Department of Home Affairs and various others. Whereas the nexus was distinctly institutionalised in the 1980 s, later it became more institutionally fragmented (Penninx 2005). Different scientific disciplines, such as anthropology, sociology, economics and political science, were involved at various times. An array of expertise was provided, such as conceptual policy recommendations by WRR and ACOM, but also more quantitative data by SCP. That policymakers generally believed in the contribution social sciences could make to the rational feasibility of social problems also played an important role (Blume, Hagendijk \& Prins 1991). This belief has made room for a more sceptical attitude towards scientific expertise in recent decades, as illustrated by controversies surrounding the parliamentary investigative committee on integration policy. There has also been a growing number of disagreements about what constitutes proper scientific research. Examples include the struggles between ACOM and WRR in the early 1990s concerning proper research methods and proper relations with policymakers; the commotion surrounding international comparative research's methodological premises following an article by the researcher Koopmans (2003), which compared Dutch integration policy with German policies; and furore about the alleged multiculturalist bias of VerweyJonker Institute (VWJ) researchers who carried out a policy evaluation study for the parliamentary investigative committee on integration policy.

There has been significant variation in the shape of the research-policy nexus in this field over the past decades. The nexus did not adhere to one of the often-formulated clichés of the research-policy nexus such as 'science speaking truth to power' or 'politics on top, science on tap'. In fact, the shape of the research-policy nexus seems to have been subject to plenty of uncertainty and controversy, just like the problem definition of immigrant integration. The nebulous problem definition combined with 
institutional incertitude about how research and policy could tame this complex social issue makes immigrant integration an intractable controversy.

This book seeks to unravel the connection between the research-policy nexus's reshaping over time and the changing manner of defining immigrant integration as a social problem. It reaches beyond a mere suggestion that the research-policy nexus played an important role in policy and research developments by analysing how and why it was structured in specific ways over the past decades and how and why it has affected the definitions of immigrant integration in research and policy. Rather, the endeavour is to explore the extent to which the research-policy nexus's varying shapes have structured how immigrant integration has been interpreted in both research and policy.

\subsection{Research-policy dialogues on immigrant integration}

Apart from the recognition that research-policy dialogues were important for research and policy developments, another issue concerns the role these dialogues had in resolving the intractable social problem of immigrant integration. The persistent controversies associated with a multiplicity of integration models in policy and research suggest that it is far from resolved. Furthermore, disagreements about the shaping of the research-policy nexus indicate how difficult it is to conduct fruitful dialogue between research and policy in the context of developing a fundamental understanding of immigrant integration - not least, who is involved, how it should be approached and why it would be a problem in the first place. In fact, policymakers have been criticised for being overly selective, simply cherrypicking from those strands of expertise that fit their problem definitions (Penninx 2005). Moreover, researchers have been challenged for being unable to reflect critically on their own problem definitions due to their entwinement with policy (Rath 2001).

This book pursues a better understanding of how the research-policy nexus could contribute to critical reflection concerning how to define immigrant integration. In this case, reflection means taking the models of integration as objects of analysis rather than as a starting point for research. Through an empirical analysis of research-policy relations and their effects on policy and research, it hopes to overcome a dreaded dialogue of the deaf at the level of problem definition and to generate insights about how to organise critical exchange between research and policy on how to define immigrant integration. This book will not resolve the ongoing debates by providing a new and superior 'model of integration.' Rather, it takes a step back to focus instead on the structure of the research-policy nexus. It will analyse how and why the research-policy nexus was structured in specific ways and how and why its different structures had certain effects on 
problem understandings in policy and research. Furthermore, this study will not determine what has or has not constituted proper scientific research. It will make no claims about the scientific character of institutes or researchers. Rather, it will take a more empirical approach to studying a myriad of research-policy nexus structures and to determining the extent to which they contributed to or inhibited critical reflection.

To encourage reflection, it is necessary to step beyond objectivist and relativist perspectives on the research-policy nexus. Objectivist perspectives further a belief that scientific research that follows proper scientific methods and norms can tame intractable controversies by producing objective knowledge about the nature of a particular social problem and countervail the irrationality of politics. This provides the foundation of the normative model of the research-policy nexus as 'science speaking truth to power' (Wildavsky 1979), which has been very influential in the social sciences overall and the policy sciences, in particular (Radin 2000). It has, however, been harshly criticised for its idealised image of science as a producer of objective knowledge claims, as well as for ignoring the many contingencies among scientific practices and policymaking (Ezrahi 1990; Hoppe 2005; Latour 1993; Mulkay 1984; Nelkin 1979). Objectivist methods ignore, for example, that running parallel to the scientification of politics is a politicisation of science (Weingart 1999). Conversely, relativism requires a more cynical take on the role of scientific research in intractable controversies. In this perspective, the contingency of scientific practices and the inherently normative character of scientific knowledge are stressed to such an extent that the role of scientific research in resolving controversies is considered negligible (Knorr-Cetina 1995; Latour 1993). Relativism often stresses the role of political ideas or the institutional interests of scientists, and argues how the production of scientific authority would be primarily a matter of discourse (Gieryn 1999).

This book adopts an empiricist approach to understanding social relations between policymakers, researchers, policy and research institutes. It also evaluates how such exchanges have promoted critical reflection on defining immigrant integration. Thus, 'research-policy dialogues' are spoken of as a way to incorporate the diverse methods for organising researchpolicy relations. Instead of adopting an ex ante model of the researchpolicy nexus, a way is sought out to empirically reconstruct the framework of the research-policy nexus during the periods that the research and policy perspectives on immigrant integration changed. Based on an empirical reconstruction of the research-policy nexus's role in these changes, an analysis is made as to how and why this nexus did or did not contribute to reflection at the level of problem definition. Moreover, it focuses on the correspondence between the research-policy nexus's structure and the definition - the framing - of immigrant integration. The attempt is to unravel to what extent the nexus was structured to contribute to critical reflection, 
or whether it played a different role in the changing problem definitions in policy and research.

Through empirical analysis of the research-policy nexus's role in policy and research developments, this book will generate insights about how the nexus can be structured so as to promote critical dialogues between research and policy at the level of problem definition. Alas, it will not resolve the controversies over immigrant integration by developing a new definition of integration or creating a normative model of the research-policy nexus. Rather, it aims to contribute to the 'situated' resolution of such controversies by actors within the structural settings of research and policy. This will be done by offering insights into how actors might organise the research-policy nexus in a way that is characterised neither by objectivism nor relativism, but rather by an effort to engage in a critical dialogue on how to define immigrant integration.

\subsection{Dutch exceptionalism?}

Although it seems plausible that social researchers played a central role in the construction of the Netherlands' models of immigrant integration, the Dutch case says little about how research can contribute to the rise or fall of specific models. In other words, is there a Dutch exceptionalism in what seems to be the co-evolution of immigrant integration research and policy over the past decades? Or, does the Dutch case reveal patterns in researchpolicy dialogues that can be found in other countries as well? In addition, what lessons can be drawn from the organisation of research-policy dialogues in other countries? Inevitably, any international comparison will run into problems, for the simple fact that integration policy and research in various countries have developed in such divergent social and political contexts and in the face of frequently differing patterns of immigration. Consequently, it is difficult to isolate factors that may be common to different nations. For instance, Germany's immigrant integration issue has been connected to its own specific twentieth-century history, which witnessed the nation's splitting into two separate states. In the UK, however, it has been connected to a national history of world-dominating colonialism (Joppke 1999b). That said, there are some commonalities that justify international comparison and may provide applicable insights for the Dutch case.

One commonality is that immigrant integration has become an issue of high politics in many Western European countries over the past decade (Geddes 2003). Just as in the Netherlands, immigrant integration is an intractable policy controversy in Germany, the UK and France. All these countries have recently experienced contention about their 'national models of integration'. Germany, after finally being recognised as a country of 
immigration, is gradually replacing its differentialist approach with a more citizenship-oriented approach à la française (Joppke 1999b). The UK, after persistently holding on to a typical British multiculturalism-on-one-island, is now gradually moving towards a more assimilative approach (Joppke 2003). France, with its clearly articulated republican model and long being considered the cradle of assimilationism, has been gradually introducing more multiculturalist elements in its policy approach. This is, in part, a result of the growing problems in the banlieues of its major cities (Favell 1998b; Schain 1995). Despite a slow but gradual process of Europeanisation, particularly in the fields of migration and anti-discrimination policies, there are persistent national differences in the approach to immigrant integration (Favell 2001). Though integration is inherently connected to issues of globalisation and cultural diversity, most policy responses seem to be driven by specific 'national models of integration'.

Moreover, in many of these countries, social research also seems to have been integral to the construction of these national models of integration. There has been, as Favell (2001) described, an undeniable association between national policy models and national research paradigms. For instance, in the UK, researchers played a crucial role in the so-called 'race relations industry' closely tied into the British colour-oriented approach to immigrant integration. Mirroring the situation in the Netherlands, various research institutes in the UK have had significant influence on policy developments across the country, including the Centre for Race and Equality Research (CRER), the Institute for Race Relations Research (IRR) and the Policy Science Institute (PSI). In line with the differentialist approach, researchers in Germany have been key to the development of a denizenship status for migrants, which means that while migrants are not recognised as full citizens, they do enjoy most social rights. In France, despite sharp politicisation of the public debate on immigrant integration, the republican model has been sustained by several 'public intellectuals'. However, this republican model has become increasingly contested in recent times, as has the nexus between intellectuals and politicians. This is most evident in typical rivalries about whether social researchers in France should be allowed to gather statistics about ethnic categories. This controversy about statistiques ethniques seems to be at the heart of the controversy surrounding the French Republican model, as well as the French way of organising research-policy dialogues in this domain.

Much debate in migration literature concerns an 'assimilationist turn' that has been taking place throughout Europe since the turn of the millennium (Joppke 2003; Joppke \& Morawska 2003). Rather than convergence by Europeanisation, the turn is compelled by internal policy dynamics in the countries themselves and, in particular, by the politicisation of immigrant integration. At the same time, there are indications of dissatisfaction with research-policy dialogues on immigrant integration throughout 
Europe. In addition to the Dutch example of questioning the credibility of immigrant integration researchers, the policy orientation of public intellectuals in France has been contested and, in the UK, the race relations industry is being replaced by a more centralised policy regime. There seems to be a correlation between the ongoing politicisation of immigrant integration and a growing disenchantment with the policy role being played by social researchers. One of the aims of this book's international comparison thus is to identify ways to organise research-policy dialogues in a more fruitful manner. How can we remedy disenchantment with and in these dialogues? And, furthermore, how can we create a more reflective dialogue on how to shape migration policies and immigrant integration research for the future? 



\section{Research-policy dialogues and the framing of immigrant integration}

The focus of this book is on how research-policy dialogues have contributed to the rise and fall of specific frames of immigrant integration. It renounces the historical-institutionalist tradition of 'models thinking'. Instead of studying the genesis and persistence of national models of integration per se, this study focuses on the much more dynamic ways researchers and policymakers frame immigrant integration in a setting characterised by a multiplicity of models of integration. Furthermore, this book focuses on the diverse ways in which research-policy dialogues can be organised within the dynamic process of problem framing. Although it is widely recognised that the research-policy nexus has been an important vector for both policy development and the development of immigrant integration research, there has been surprisingly little empirical research into how and why the nexus has played such a role. The aim here is to contribute to theory-building on how research-policy dialogues influence the process of problem framing. 'Research-policy dialogues' are consciously spoken of in order to capture the often dynamic ways in which research-policy relations can be configured.

The theoretical framework on which this study rests will incorporate insights from various disciplines - namely, sociology, science studies and policy sciences. This melding will be based on the theoretical groundwork of Bourdieu's structuralist-constructivist perspective. Incorporating insights from the more generic disciplines in the study of immigrant integration policies is a deliberate choice; it minimises chances for contamination between the theoretical perspective of this study and the various frames and research paradigms present in the field of immigrant integration research. Furthermore, it contributes to opening up the thriving yet, at times, closed field of immigrant integration research by connecting it to the more generic theories developed in the academic disciplines of sociology, science studies and policy sciences. 


\subsection{Structuralist-constructivism: Beyond 'models thinking' and radical constructivism}

Before elaborating on the conceptual framework of the structuralist-constructivist perspective, it will first be positioned within broader contemporary literature from migration studies.

\subsubsection{Beyond 'models thinking' in immigrant integration research}

The structuralist-constructivist perspective parts with more historical-institutionalist ones that have acquired great resonance in contemporary migration literature. This has manifested itself most obviously in the so-called 'models' literature, which discusses the genesis and persistence of, mostly notably, national models of integration. A classic reference in models thinking is Brubaker's 1992 book Citizenship and nationhood in France and Germany. Juxtaposed here are the models of citizenship that provided the foundations for the integration policies in these countries: an assimilationist approach in France and a differentialist approach in Germany. Whereas the Germans stressed exclusive membership of the German community based on ethnic ties (ius sanguinis), the French adopted a more inclusive model oriented towards full citizenship for everyone born on French soil (ius soli). As a true historical-institutionalist, Brubaker shows how the historical conditions in both countries led to the construction of these national models: a well developed cultural and apolitical sense of national belonging in Germany versus the state-centric tradition of nationbuilding in France.

Models thinking has resonated widely in migration studies. Take, for instance, the work of Joppke (1995) who has used the national model as a starting point for comparative studies of immigrant integration, although his more recent work has become more explicit about how countries are increasingly deviating from their traditional models. Or Ireland (1994) who, in a comparative study of France and Switzerland, posits that national institutional conditions provide the best explanation for the type of policies developed. In his Dutch-German comparison, Koopmans (2003) takes the differences in national models as a point of departure for evaluating the effectiveness of the Dutch and German approaches. The notion that Germany denounces the Dutch multicultural model as a failure can also be found in the work of Sniderman and Hagendoorn (2005).

One of the reasons models have gained such wide acceptance in migration studies (and in other sectors) is that they reduce complexity. Models can simplify the otherwise highly complex and contested matter of immigrant integration. They can also help construct international comparative studies to assess processes of convergence or divergence between various European countries. Furthermore, by comparing ideal-typical models with 
specific periods, modelling can provide insights into a country's history. In this context, Castles and Miller (2003) and, in their footsteps, Koopmans and Statham (2005) have extended Brubaker's dichotomy into a fourfold typology of integration models: civic-assimilationism, cultural pluralism, ethnic-differentialism and civic-republicanism. An important distinction in Brubaker's historical-institutionalist modelling is that it concerns idealtypes that can be used for studying country cases, rather than models to be seen as representative of national approaches per se.

That said, the danger of models is that they are not just taken as tools for international comparison or for understanding historical periods. When a model begins to shape our understanding and beliefs about policies it becomes more than just a model - it becomes a historical reconstruction of policy rather than a model of it. Models then take the place of adequate historical analysis. In social science literature, this has often led to instances where a model is 'blamed' for the success or failure of a particular policy approach. For example, various authors have blamed the Dutch multicultural model for the alleged failure of immigrant integration in the Netherlands (Koopmans 2003; Sniderman \& Hagendoorn 2007).

In addition, models tend to oversimplify policies and overemphasise the alleged coherency and consistency of these policies (Bertossi \& Duyvendak 2009). Policy practices tend to be far more resilient and diverse than most policy models would suggest. For instance, in Dutch as well as in French literature, there have been many references to differences between how policies are formulated at the national level and how they are put into practice at the local level; some even talk of the decoupling of national and local policies in this respect (Favell 1998; De Zwart 2007; Poppelaars \& Scholten 2008). In fact, even when policymakers claim to operate according to a specific policy model, their reasons for doing so may be more pragmatic and flexible than the policy model itself in its ideal-typical form. For instance, the reason some politicians in the 1980s framed immigrant integration in terms of the multicultural model may have more to do with their fear of anti-immigrant parties playing the race card than with their multicultural policy beliefs (Penninx 1988; Scholten 2007).

A structuralist-constructivist perspective gives way to a much more empirical and dynamic approach to immigrant integration. Here, the so-called 'models of integration' are the object of empirical analysis, rather than simply a starting point for analysis. In fact, the association between researchers and certain models of integration is one of the central issues that will be problematised in this book. Thus, models are taken as specific frames that may emerge in policy and research, and the primary objective of this study is to analyse how and why these frames rise and fall, rather than to establish whether a particular frame is true or false. In short, this book appreciates the dynamics behind the models more than the theoretical value of the models themselves. 
This does not, however, imply a radical-constructivist approach to immigrant integration. Such would deny the possibility of defining immigrant integration in any meaningful way and deny any empirical relevance of models of integration. However, it cannot be ignored that international migration and growing cultural diversity have been affecting contemporary societies in the Netherlands and other European countries in very real ways, or that governments have made very real efforts to develop policies for coping with these social effects. Indeed, immigration has had an impact on key social institutions, such as the welfare state, and has been challenging social cohesion and traditional ideas about national identity. In some cases, it has led to an ethnicisation of the underclasses. In fact, immigrant integration has now become an urgent policy concern for most European countries. In this sense, the structuralist-constructivist perspective adopted in this book is not so much meant to deconstruct immigrant integration policies as mere discourse but rather, to develop better understanding of how and why specific discourses emerge and change over time.

\subsubsection{Outline of a structuralist-constructivist perspective}

Structuralist-constructivism goes a step beyond models thinking and relativism in the study of social structures and social problems. It combines a constructivist view on social structures, such as science and policy, with a structuralist perspective on the construction of problems like immigrant integration (Bourdieu \& Wacquant 1992: 11). It adopts an empirical position when examining how scientific research and policymaking are constructed in actual social relations and the practices of actors in these fields. It also takes an empirical approach to how these structured fields influence the way actors socially construct the world around them, for instance, how they define social problems like immigrant integration, or how they conceptualise the research-policy nexus.

Structuralist-constructivism is based on specific ontological and methodological premises. In terms of ontology, it sees the research-policy nexus and problem frames as products of structured social relations. This means that the distribution of power and the rules of the game manifested in dealings between actors in a certain domain are considered explanations for how and why these actors construct the research-policy nexus and frame problems. In terms of methodology, structuralist-constructivism promotes an empirical approach to the study of the research-policy nexus and problem framing. Only by studying the social practices of actors and the social relations between then can we begin to understand how and why the research-policy nexus is shaped the way it is and how and why resulting problems are framed as they are. In short, structuralist-constructivism neither nullifies nor reifies the research-policy nexus or problem framing; rather, it 
defines both as 'relational', being products of actual social practices and relations (Bourdieu 1975; Bourdieu \& Wacquant 1992).

Two literatures will be combined to study the research-policy nexus in terms of problem definition. The first was developed by Rein and Schön to study how actors frame issues in inherently selective and normative ways. This perspective recognises that social problems are not merely 'out there' and dictated by facts but rather, that they are socially defined or 'made real'. Problem situations - especially those that are complex, multifaceted and deal with various normative issues - are often characterised by a multiplicity of possible realities or 'frames' (Goffman 1974; Rein \& Schön 1994). A problem frame will require naming specific facets from problem situations and an inherently normative way of framing them into cognitive stories about what is going on, who is involved, why it is going on and what could or should be done to provide a solution. For instance, different frames of immigrant integration can focus on different problem facets (e.g. social-economic or social-cultural issues) and different groups or categories (e.g. foreigners or minorities); they can tell different stories to explain what is going on (e.g. discrimination or inadequate citizenship); and they can make different normative leaps from 'is' to 'ought' (e.g. preserving social cohesion or facilitating cultural diversity).

Rein and Schön's approach to problem framing takes a relational perspective, which recognises the influence of the structural setting in which framing takes place. They refer to frames as being connected to particular institutional forums that induce actors to name and frame a problem situation in a specific way (Rein 1986: 12). Their discussion of frame reflection raises a crucial question: when do the structures of such forums and the connections between them influence actors to reflect on the frames or on possible alternatives? Rein and Schön argue that such structures must generate an openness towards alternatives, empathy towards other frames, a critical attitude of one's own frame and a willingness to adapt when necessary, as well as a certain degree of trust among the actors involved in critical frame reflection (Rein \& Schön 1994: 37).

Framing, however, does not provide a relational perspective on the institutional forums themselves to explain how and why they are structured in specific ways. In fact, the notion of institutional forums is used rather loosely in the works of Rein and Schön. Moreover, it does not hone in on research and policy as institutional forums. Consequently, the frame perspective applied in this book will be combined with a more developed relational perspective on research and policy and on the structure of their mutual relations. This book adopts Bourdieu's interpretation of science and policy as 'fields' of structured social relations that are constantly at stake not only within the fields themselves, but also in between them. This notion of field has been developed more fully in the institutional sociology of science (Hess 1997: 52). Here, literature has evolved on 'boundary work', 
which examines how actors within certain positions demarcate a field and coordinate its social relations with other fields. Fields and boundary work are closely related in a structuralist-constructivist perspective: what is at stake at the boundaries of a field will also impact the field itself. For instance, structural changes within a field can be affected by changes along the boundaries with other fields and vice versa, such as when an institute obtains authority or funding for doing research, which will, in turn, reinforce its position within the field.

Boundary work and ongoing processes of redefining field structures in research and policy can combine in various ways, producing different structural nexuses between both fields. These 'boundary configurations' are a structural product of boundary work and structural developments within fields. They can also be a source of change within both fields, as specific relations are established between them and they generate different degrees of autonomy. Various theoretical models of boundary configurations are distinguished, such as enlightenment, bureaucratic, engineering and technocratic configurations (Hoppe 2005; Wittrock 1991). In this respect, the literature clearly departs from objectivist approaches that stick to one universal standard model of research-policy relations, as well as from relativist approaches denouncing the idea that we can speak of boundary configurations, field autonomy and structural relations among the fields in any meaningful terms.

The literature about fields and boundary configurations will be connected to Rein and Schön's ideas about problem framing and frame reflection. As the authors have argued, boundary configurations can be a consequence, as well as a source, of changes in field structures. They can, for instance, either challenge or reinforce the structures or institutional forums in which a particular frame is embedded. They can generate either positive feedback towards change or negative feedback to induce stability (Baumgartner \& Jones 1993). As such, boundary configurations can offer strategic opportunities for change in research as well as policy, as suggested in Fischer's (1993) analysis of the politics of expertise, showing how research-institutes or think tanks play a role in the war of ideas among different political groups. Furthermore, the structural effects of boundary configurations can generate structural conditions that challenge susceptibility to a particular frame. For instance, Guiraudon (1997) has shown how keeping policymaking behind closed doors - in research institutes - has contributed to a policy framing that allowed for the extension of migrant rights in various countries.

Frame reflection requires boundary configurations to be structured in specific ways. This involves what Rein and Schön (1994: 165) describe as 'design rationality', which can be productively applied to the structure of research-policy relations. In order to meet the demands of design rationality, boundary configurations have to generate openness, empathy towards 
alternative frames, critical reflection on these frames, a willingness to adapt frames if necessary and a certain amount of trust in order to engage in critical debates. If these criteria are met, boundary configurations can lead to critical frame reflection at the level of problem framing. If not, they can still influence frameshifts, though in a way other than frame reflection. Through the concept of framing, however, such non-reflective interaction between research and policy is perceived as a dialogue of the deaf.

Finally, problem framing and the construction of boundary configurations in the domain of immigrant integration cannot be divorced from the broader social context. From a structuralist-constructivist perspective, context is not merely an external constraint on problem framing and boundary work. The selective and normative manner in which actors frame an issue also involves selective and normative ways of perceiving the social context. For instance, developments in the problem situation do not simply determine problem framing, but will be mediated through the problem frames of actors, who then select and interpret the developments in certain ways. Furthermore, the boundary work practices of actors can be affected by structural developments beyond the scope of immigrant integration research and policy. For instance, macro-institutional developments in the structure and culture of politics and policymaking can affect the boundary work of actors within the domain of immigrant integration, though they can also be mediated by changes in their positions within the research and policy fields.

\subsection{Framing immigrant integration}

As controversies in the Netherlands over immigrant integration demonstrate, there is persistent disagreement about what immigrant integration is, why it is important, who is involved, who is to blame and what is to be done about it. In contrast to the traditional belief in objective and rational problem definitions, this issue appears to be characterised by a multiplicity of problem frames. How, then, can we grasp those situations, theoretically and conceptually, when the problem framing itself is contested?

\subsubsection{The sociology of social problems}

The frame concept has its theoretical roots in the sociology of social problems. Goffman (1974) was one of the first to develop this idea and did so within a more symbolic interactionist strand of theory. He studied how actors construct answers to the question 'What is going on here?' within actual social practices, such as everyday interactions with other actors. He coined the term 'frame' to describe how actors attribute meaning to reality and how they position themselves in it. Through frames, actors create a 
subjective order out of an ambiguous and complex reality. Frames also allow actors to understand what their position in this reality is and how their actions in response to it should be guided.

Rein and Schön (1994) elaborate the frame concept by putting it into a more cognitive and structuralist perspective. They position frames on a cognitive level in terms of how actors have learned to define and understand a situation. They do, however, acknowledge that human cognition is inherently entwined with more subjective images and normative appreciations. Rein and Schön thus define frames as 'underlying structures of belief, perception and appreciation' (ibid.: 23). Frames are generally 'tacit' or unknown to actors themselves (ibid.: 34). However, they do play an important role in actual social practices: to provide a 'way of selecting, organising, interpreting, and making sense of a complex reality to provide guideposts for knowing, analysing, persuading and acting' (ibid.: 32).

Furthermore, Rein and Schön (ibid.) argue that 'framing [...] always takes place within a nested context'. This nestedness involves various structural or institutional forums, each with their own rules of the game and a distribution of social roles (Rein 1986: 13). In fact, they argue that this affects how actors will frame an issue; 'the institutional context may carry its own characteristic perspectives and ways of framing issues [...] or it may offer particular roles, channels and norms for discussion and debate' (ibid.: 12). Varying institutional forums elicit different 'criteria by which judgments are made about the legitimacy of participants and their standing as participants in the policy conversation' (ibid.: 13). This approach resembles Gusfield's (1980) sociology of social problems, which focused both on what he described as 'the culture of public problems' and 'the structure of public problems'. For example, in his research on car accidents, Gusfield (ibid.) demonstrated how the structure of this problem - involving a dominant National Safety Council, insurance companies and industries influenced the culture of this problem - focusing on unsafe drivers ('the drinking driver') rather than on unsafe cars or roads. This is reminiscent of Schattschneider's (1960) dictum that every structural organisation involves a selective mobilisation of bias.

\subsubsection{Naming and framing reality}

So, 'What is going on here?' The literature on the sociology of social problems is tasked with distinguishing several facets of how problem situations are made sense of in problem framing. This involves the use of specific discourse or language for naming the problem situation, defining the groups or categories that are involved, a causal story to explain the problem and a normative perspective for suggesting what could and should be done about it. 
First, frames name an issue in terms of concepts and metaphors. Naming - wording - reality is the first step to framing reality. Language is more than a neutral description; it not only describes but also makes reality. According to Edelman (1988: 9), 'the language that interprets objects and actions also constitutes the subject'. Concepts can give social meaning to particular situations, especially when their historical usage has allowed them to develop a 'loaded' meaning that is widely recognised. They can make subjects 'tangible' or 'real' by referring to meanings that are more commonly used (Parsons 1995: 180). Concepts can therefore become central carriers or devices in the 'dynamics of knowledge', transferring meaning among various issue domains (Maasen \& Weingart 2000). They can become 'generative' metaphors (Rein \& Schön 1994; Stone 2002 [1988]) as they project a particular historically developed meaning onto a new situation. Furthermore, specific concepts and metaphors can play a central role in the framing of policy problems. In fact, as Edelman $(1977,1988)$ has argued, the construction of symbolic meaning can become the centre of the 'political spectacle' rather than actual problem solving. For instance, as politicians try to convey positive images about particular policy frames, and despite opponents highlighting the deficiencies of such policies, it is quite possible that words will succeed where policies actually fail (Edelman 1988).

Secondly, naming issues involves the social classification of relevant groups or categories (Yanow 2000). Defining whose problem it is, and why it is a problem, is an important aspect of issue framing. Social classification involves defining groups using a particular group structure or categories that share a set of characteristics. For instance, it can make a great difference whether integration concerns specific ethnic or cultural groups (e.g. Turks or Muslims) or specific categories (e.g. guest workers, foreigners or aliens). As Schneider and Ingram $(1993,1997)$ have shown, the classification of groups or categories is a far from unambiguous or neutral process. They draw attention to how social classification conveys public images and perceptions of power positions of specific groups or categories. For instance, it can pose both significant political risks or opportunities in terms of burdening the advantaged and independent or, in constrast, providing benefits to contenders and deviants.

Thirdly, the framing of a problem situation requires not only that relevant facets and groups and categories be named, but also that the names be inserted into an intelligible, convincing story of how an issue can be explained. According to Rein and Schön (1994: 26), stories 'construct a problem out of the vague and indeterminate reality'. Stone (1989) has elaborated this, explaining the concept of causal story as a narrative about what causes a problem (responsibility) and the extent to which a problem can be tamed (control). In this respect - and debates about whether reality is causally determined aside - it is important that these stories socially construct 
causality in a way that should be comprehensible and convincing rather than empirically accurate. The stories arise from social struggles over causality.

Finally, frames are not only about what 'is' but also about what 'ought to be'. Rein and Schön (1993: 148) label this the 'normative leap from is to ought'. Frames, then, influence not only knowledge and understanding, but also social action. The normative leap will often involve core values that resonate broadly in society and that can be 'communicated directly and simply through image and rhetoric'. Core values include equality, equity, liberty, progress, solidarity and patriotism (Baumgartner \& Jones 1993: 7). Fischer and Forester (1993) posit frames not only as constructing a reality, but also a call for action upon this reality. This has been referred to as the performative function of frames. For instance, framing society as a multicultural society will also appeal to values such as cultural equality and equity and elicit a call for action to effectuate such values. In contrast, framing society with values like patriotism compels a vastly different normative leap from what is to what ought to be.

\subsubsection{Frames of immigrant integration}

As argued above, there has been a preponderance of studies of 'national models of integration' in contemporary migration literature. In this book, these models will be conceptualised as ideal-typical frames rather than as supposedly empirically valid depictions of actual policies. For the empirical analysis of the attributes of frames of immigrant integration (terminology, social classification, causal theories and normative perspective), the models employed are those developed by Castles and Miller and elaborated further by Koopmans and Statham. Castles and Miller (1993) distinguish three models: differentialist, assimilationist and multiculturalist models. Koopmans and Statham (2000) added a universalist model to this framework. Furthermore, in a recent publication, Castles and Miller (2003) also distinguish transnationalist and post-nationalist models, which reach beyond the scope of the nation-state.

\section{Assimilationism}

Assimilationism, together with multiculturalism, is one of the most written about models of immigrant integration. It has its roots classical sociology and, in particular, in Park's Chicago School of Sociology and his race relations cycle, which distinguishes between several 'stages' of assimilation (Park 1928).

Assimilationism names and frames immigrant integration mainly through how migrants adopt the culture of native society (Gans 1997; Park \& Burgess 1921). It phrases the problem situation primarily in terms of the 'social-cultural adaptation' of immigrants and the preservation of 'social 
cohesion'. Classifying the groups or categories involved is an inherent dilemma in assimilationism. On the one hand, it tends to name groups in culturalist or ethno-cultural terms, as is the case for the German definition of the national Volksstaat (Brubaker 1992). On the other hand, the identification of culturally deviant groups may lead to the reification, rather than bridging, of cultural differences. This reification can be avoided if immigrants are defined using social categories (for instance, as newcomers) rather than groups. Conversely, public discourse is more likely to emphasise ethno-cultural groups. In causal terms, immigrant integration is framed as a process in which social-cultural adaptation is a condition for preserving national social cohesion, as well as for the amelioration of the social position of migrants in their new societies. Therefore, migrants should be willing to adopt national norms and values and national institutions should be effective in terms of including migrants. Concerning its normative perspective, assimilationism frames immigrant integration in terms of national identity and national social cohesion in relation to the viability of the national community.

\section{Multiculturalism}

Multiculturalism is generally posited as the opposite of assimilationism, as it stresses cultural pluralism and a more culturally neutral, open form of citizenship (Koopmans \& Statham 2000). However, an important point of convergence between assimilationism and multiculturalism lies in their focus on the nation-state. In multiculturalist theory, the nation-state is redefined in terms of the recognition of being a multiculturalist state (Vertovec 2001). This is best illustrated by the British form of 'multiculturalism-onone-island' (Joppke 1999).

Multiculturalism names immigrant integration in terms of cultural diversity and the need for emancipation of groups of varying cultural backgrounds. Whereas adaptation involves finding commonalities between individuals in society, multiculturalism searches for compatibilities between groups and for tolerance of those facets of social life that groups do not have in common. Groups are socially constructed based on their cultural, ethnic, religious or racial traits, to name a few. Political theorists Kymlicka (1995) and Parekh (2000) have argued that accommodation of cultural differences between groups may even require the diversification of social and political rights for distinct groups. The causal theory that underlies most multiculturalist thinking is that the only way to accommodate cultural pluralism is to recognise cultural diversity and to differentiate policies for particular cultural groups (Taylor 1992). As an example, group-specific policies have to be developed in various spheres, including general policy spheres such as education and labour. Finally, multiculturalism contains a normative perspective that cultural diversity is a value in itself - a facet of the ongoing process of modernisation - and that government interference 
should be limited (i.e. states should be tolerant) as the cultures themselves will determine the identities of cultural group members.

\section{Differentialism}

The third model, differentialism, is also known as 'ethnic segregationism' (Castles \& Miller 1993) and it involves the institutionalisation of differences - in a way, 'living apart together'. Cultural diversity is institutionalised in the form of parallel societies, similar to the South African apartheid regime, the Indian caste structure or the Dutch history of pillarisation (Lijphart 1968). Aside from these radical variants of differentialism, the model has been applied in more subtle forms, as in the accommodation of temporary foreign labourers in many Western European countries.

In this model, immigrant integration is named primarily in terms of accommodating differences between groups that are, as much as possible, autonomous or sovereign within their own community. In fact, the term 'integration' is unlikely to be used in this model, as integration is to be achieved only in those domains where coordination between groups is necessary. This model classifies migrants in ways that stress their status as distinct national, ethnic, cultural or religious groups. In Germany, for example, migrants have long been labelled 'Ausländer', thus being defined by national origin so as to emphasise their non-German status. The causal story that underlies differentialism stresses either the absence of a need to integrate (e.g. because migration is considered temporary) or the unfeasibility of integration (e.g. because of essential differences between migrants and natives). In respect to the latter, France has witnessed zealous discussions on the 'unassimilabilty' of migrants - Muslim migrants, in particular - thus advocating differentialist policies similar to those in Germany. Finally, values and norms found in the differentialist model are not unlike those of assimilationism. Both favour the idea of organic communities and stress bonding with the community. In the assimilationist model, this occurs in the national community; with differentialism, bonding occurs within the separate community.

\section{Universalism}

Koopmans and Statham (2000) distinguish a 'universalist' or 'civic-republican' model that adopts a more liberal egalitarian view on immigrant integration. It differs from both multiculturalism and assimilationism (especially in its meaning of acculturation) in that it is not culturalist; the focus is not on either commonalities or compatibilities between groups in cultural pluralist societies. In fact, it is adverse to the institutionalisation of both majority and minority cultures. Civic republicanism is more oriented towards individual citizenship in a (culturally neutral) society.

In this model, immigrant integration is named in colour-blind and individualist terms, such as 'citizenship' or 'participation'. Furthermore, 
universalism focuses primarily on the social-economic and political-legal spheres of integration rather than the social-cultural ones. Culture and religion are considered issues belonging to the private realm. In the public realm, stress is put on individual participation in spheres such as labour, education, housing, health and other colour-blind sectors. Migrants are defined as categories of individuals, for instance, immigrés in France or allochtonen in the Netherlands, thereby letting the culture of these groups recede into the background. The causal theory underlying universalism underscores the need for migrants to be able to stand on their own feet as citizens of society, especially in terms of social-economic participation. In this respect, combating discrimination and the effectiveness of institutions, such as education and labour, at including migrants are important issues in universalism. In normative terms, universalism contains a liberal egalitarian perspective on society, whose core values are good citizenship and equality.

\section{Transnationalism and post-nationalism}

In contrast to the models described thus far, transnationalism and post-nationalism do not focus primarily on the nation-state. Nonetheless, these models can be used by actors to frame how migrants are to be incorporated into society. Transnationalism links migration and integration to the process of internationalisation, studying, for instance, the formation of transnational migration 'bridges' and the formation of transnational migrant communities (Faist 2000; Kivisto 2001). This is manifested in areas like the common European migration policy that has been formulated over the past decades. Post-nationalism represents a more cosmopolitan school of thought, linking migration and integration to globalisation, including discussions of the transformative effects on nation-states (Jacobson 1996; Sassen 1998; Soysal 1994).

These models often name and frame integration in non-national terms, such as transnational or post-national citizenship. Moreover, they often stress transnational developments such as links between countries of origin and destination and the development of a universal human rights discourse. Migrants are defined in terms of groups extending beyond the scope of one national state, such as transnational communities that straddle two worlds, or as universal categories, such as universal personhood. Types of causal theories developed in these models include ideas that migration is posing a challenge to the nation-state and that new (non-national) modes of integration are being invented. Through these perspectives, immigrant integration generally also involves the adaptation of national society and its central institutions to the emergent realities of cultural diversity and migration. For example, the models challenge dominant ideas about national citizenship, questioning the issue of exclusive loyalty to one nation. Finally, the normative perspective of transnationalism and post-nationalism 
is oriented towards internationalisation and globalisation as facets of the normative process of the modernisation of societies. Concerns about migration and integration are simply manifestations of the process.

\subsection{The research-policy nexus}

The social construction of problems is considered inherently related to the situational setting in which framing takes place. This book focuses precisely on the nexus between immigrant integration research and policy as part of that structural setting. Required thus is a placement of problem framing in the context of the structural setting of social relations within and between research and policy. Or, to use Gusfield's (1980) terms, we have to pay attention to the culture and structure of public problems - in this case, the structure of research, policy and their relations.

\subsubsection{Social research and policymaking as fields}

From a structuralist-constructivist perspective, both social-scientific research and policymaking are defined as fields of structured social relations (Bourdieu 1975, 2004; Bourdieu \& Wacquant 1992). The actors' positions and the rules of the game within a field involve distributions of power 'capital' - among actors. This can involve economic capital (resources), as well as social capital (networks), cultural capital (knowledge) and symbolic capital (authority, legitimacy) (Bourdieu \& Wacquant 1992: 17). According to Bourdieu, the distribution of capital defines a field's structure, as it determines the distribution of positions among actors and determines the rules of the game. From this point of view, a field is not a level playing field: it contains a specific distribution of capital. For instance, in his study of intellectuals, Bourdieu (1988) shows how actors are driven by the determination to distinguish their position as intellectuals from other actors (non-intellectuals) and define the rules of being a good intellectual in such a way that it provides them with symbolic capital (authority) within the structure of the intellectual field.

Field structures are inherently dynamic. They provide spaces of conflict and competition that advocate change as well as stability. Some actors will be driven to change a field structure, while others will be more inclined to reproduce it, depending on the distribution of capital within. Some actors will be driven to construct a field structure so that it includes and excludes specific actors. Thus, the boundaries between one field structure and that of other fields are constantly at stake within the field itself (Bourdieu \& Wacquant 1992: 97-104).

It is important to note that, from a structuralist-constructivist point of view, field structures do not exist beyond the actors in a field. Rather, 'it is 
in the relationship between the various agents that the field and the relations of force that characterise it, are generated' (Bourdieu 2004: 33). This means that a field structure is not seen as an exogenous constraint on actor relations, but as their endogenous product. Field structures constrain and enable social relations through what Bourdieu (1988) describes as relatively enduring dispositions - the 'habitus' of actors. Habitus does not involve a form of conscious rule-following but rather, a sort of 'feeling' or 'sense for the game'. It is in the regularities of the actors' habitus that field structures exist. Furthermore, the habitus reflects the capital an actor possesses. For instance, becoming an 'intellectual' would require an intensive conversion of economic capital (resources, time) into social, cultural and symbolic capital in order to obtain a position within the intellectual field and to get a sense of the game. Once such a position within the field is obtained, the habitus of the actor will constrain inclinations to transform the field structure in a way that would produce negative outcomes in terms of the distribution of capital.

In this way, the field perspective shifts attention from how actors within these fields frame issues, such as immigrant integration, to their habitus, their structural positions and their affinity to the rules of doing research and making policies. The habitus and accompanying field structures can only be established through empirical analysis of the actual social relations or practices of the actors within both fields. They cannot be assumed as is the case in objectivist analyses, or ignored as occurs in relativist analyses. The focus is on scientific research and policymaking as 'crafts' or 'arts' (ibid.: 38).

The pure universe of even the purest science is a social field like any other, with its distribution of power and its monopolies, its struggles and strategies, interests and profits, but it is a field in which all these invariants take on specific forms. [...] As a system of objective relations between positions already won (in previous struggles), the scientific field is the locus of competitive struggle, in which the specific issue at stake is the monopoly of scientific authority, defined inseparably as technical capacity and social power, or, to put in another way, the monopoly of scientific competence, in the sense of a particular agent's socially recognized capacity to speak and act legitimately (i.e. in an authorized an authoritative way) in scientific matters. (Bourdieu 1975: 19)

The policy and research fields are characterised by their own distinct forms of capital, often described as political capital and scientific capital (ibid.: 34). Both are forms of symbolic capital that require recognising the authority and legitimacy of actors to make policies or to be recognised as 'scientific'. As forms of symbolic capital, they are 'based on knowledge and 
recognition [...] which functions as a form of credit, presupposing the trust or belief of those who undergo it because they are disposed to give credit, belief' (ibid.). Depending on a field structure, some actors will be more effective in terms of defining scientific and political capital in a way that works in their favour. Or, there will be those "who manage to impose the definition of science that says what the most accomplished realization of science consists in having, being and doing what they have, are and do' (ibid.: 63). As such, policy and research can be perceived as a 'structured field of forces', characterised by 'struggles to conserve or transform this field of forces' (Bourdieu 2004: 33). In other words, 'the definition of what is at stake in the scientific struggle is one of the things at stake in the scientific struggle' ibid.: 63).

This structuralist-constructivist perspective on research and policy also involves a structuralist-constructivist perspective on how problems are framed or how 'knowledge' and 'truth' are produced within these fields. Truth is defined in relational terms, which means that what passes as objective knowledge is what gets defined as such in the context of the structure and the distribution of power in a field. 'The objective truth of the product even in the case of that very particular product, scientific truth - lies in a particular type of social conditions of production, or, more precisely, in a determinate state of the structure and functioning of the scientific field' (Bourdieu 1975: 19). The epistemological and social characteristics of knowledge production are considered inherently entwined. 'Epistemological rules are nothing other than the social rules and regularities inscribed in structures and/or in habitus, particular as regards the way of conducting a discussion [...] and settling conflict' (Bourdieu 2004: 71). Thus, struggles over the structure of field structures are considered inherently related to struggles over what is framed as 'the truth' within these fields.

\subsubsection{The co-evolution of research and policy}

Fields are inherently dynamic, as the positions in a field and its rules of the game are constantly at stake. Moreover, the dynamics in field structures are related to the dynamics in other fields. Bourdieu argues that changes in a field structure may be difficult to achieve from within because the positions and rules that define it carry a particular distribution of capital that tends to resist change. Interaction with other fields therefore constitutes an important driver of changes in a field structure. There are, however, no 'transhistoric laws of relations between fields' (Bourdieu \& Wacquant 1992: 109-110). The connections between fields must be studied empirically by analysing the actual social relations among the actors from the fields in question.

The theoretical notion of 'boundary work' was coined by the institutional sociology of sciences to refer to social practices occurring on the 
boundaries of fields. Stressed is the fact that these boundaries are not a given. Examined here is how actors create social boundaries in their social practices to distinguish one field (its structural positions, rules of the game and species of capital) from others. Gieryn (1983: 782) defined boundary work as the attribution of 'selected characteristics to the institution of science (i.e. to its practitioners, methods, stock of knowledge, values and work organisation) for purposes of constructing a social boundary that distinguishes some intellectual activities as "non-science". This notion allows for an empiricist study of relations between fields, studying actual boundary work practices instead of doing boundary work with ex ante models or 'laws' governing relations between fields such as science and policy.

However, boundary work is more than a recognition of fields through a distinguishing of field structures. It does not simply mean dividing up fields like science and politics to different degrees, as the somewhat onedimensional term 'boundary' may suggest. Shapin (1992) and Halffman (2003) have drawn attention to the dual nature of boundary work as something that requires not only delineating fields, but also coordinating their mutual relationship - a coordination that will necessarily result in the demarcation of roles and tasks. Elaborating on a definition from Shapin (1992: 335), Halffman (2003: 241) defines boundary work as follows:

Boundary work defines a practice in contrast with other practices, protects it from unwanted participants and interference, while attempting to prescribe proper ways of behaviour for participants and non-participants (demarcation); simultaneously, boundary work defines proper ways for interaction between these practices and makes such interaction possible and conceivable (coordination).

Whereas Shapin and Halffman refer to boundary work as the demarcation and coordination of practices, this book slightly adapts the definition to apply to the demarcation and coordination of fields. The structuralist-constructivist perspective adopted here focuses more on the structural properties of fields and their relations (such as cause and effect) to how boundaries with other fields are constructed. Shapin and Halffman have a more symbolic-interactionist conception of boundary work, although they do (more so than Gieryn) extend their analysis of boundary work beyond the realm of discourse.

Boundary work reveals an important facet of the transformation of field structures precisely by demarcating and coordinating relations with other fields. In this context, much has been written about the co-production or co-evolution of science and politics (Ezrahi 1990; Jasanoff 2004; Nowotny, Scott \& Gibbons 2001; Shapin \& Schaffer 1985). Nowotny et al. (2001: 245) referenced the increasing transgression of science-politics boundaries and the contextualisation of science, which means that science 
not only speaks to society but society also speaks back to science. According to Shapin and Shaffer (1985: 332), there is a 'conditional relationship between the nature of the polity occupied by scientific intellectuals and the nature of the wider polity'. Ezrahi (1990) has described the rise of modern science in relation to modern societies' growing demands as an instrumental means to sustain administrative control; in this regard, science is an important political resource for depersonalising and depoliticising ideological state control, thereby legitimising modern liberal democratic politics.

In this context, Bourdieu points to the conversion of scientific capital into other sorts of capital in the relations between the fields of research and policy. This concerns a conversion into economic, social or cultural capital (Bourdieu 1975: 25), as well as other sorts of symbolic capital (Bourdieu 2004: 55). Apart from 'strictly scientific authority' (Bourdieu 1975: 57), there would also be scientific capital that is more profoundly related to other sorts of capital, or capital that involves 'power over the scientific world which can be accumulated through channels that are not purely scientific [...] and which is the bureaucratic principle of temporal powers over the scientific field such as those of ministers and ministries, deans and vice chancellors or scientific administrators' (ibid.). This can refer to, for example, the accumulation of scientific capital through the acquisition of research funding or a scientific expert exercising policy influence rather than performing scientific research.

Organisations that have found a niche in the interaction between the fields of scientific research and policy often play a central role in the boundary work and co-evolution of these fields. These 'boundary organisations' (Guston 2000; Miller 2001) can come in many shapes and sizes, including think tanks (Stone 1998) and so-called 'universities without students' (Weaver 1989), as well as certain foundations with combined social and scientific purposes, private consultancy firms or government contractors, government research bureaus (Stone \& Garnett 1998) and advocacy think tanks (Abelson 2002). Though these organisations are often portrayed as 'bridges' or 'transmission belts' between research and policy, they generally take a more active role (and interest) in boundary work. They are generally hybrids of the structures of both fields, combining elements of both science and politics (Miller 2001). However, they derive much of their credibility from clearly demarcating science and politics and by positioning themselves somewhere in between.

Their credibility is grounded in the 'two worlds' metaphor. [...] [I]t is in the interest of think-tanks in general to maintain the myth of the distinction between knowledge and scholarship on the one hand, and politics, policy and interests on the other. If policy research institutes are 'above' politics they are not a threat to democracy. 
Portrayed passively as a bridge or a transmission belt from the scholarly domain, the metaphor of two worlds gives them a safe distance from politics and protects their credibility and charitable status. (Stone 1998: 121)

Boundary organisations are able to occupy this niche because they have a capacity to convert scientific and political capital. They often hold positions within both fields and also must find ways to functionally blur the rules of the game within both fields in a way that allows for interaction between them. For instance, a scientific advisory body has to possess authority in both politics and science in order to be able to provide counsel that is considered simultaneously useful in politics and credible in science. Internally, boundary work also involves a degree of balancing, in an effort to maintain authority within both fields. In relation to the broader fields of research and policy, boundary organisations can constitute an important part of the institutionalisation of the research-policy nexus. Every boundary organisation will adopt a particular method for demarcating and coordinating research and policy that, once the boundary organisation is established, tends to institutionalise. Clearly, in this process of institutionalisation, boundary organisations not only play a central role in research-policy relations, but also in organising them.

\subsubsection{Boundary configurations of the research-policy nexus}

Similar to the structures of fields, the research-policy nexus - or 'boundary configuration' - will have its own structural distribution of positions and roles on both sides of the boundary, as well as its own structural rules of the game concerning relations across the boundary. As such, a boundary configuration structures the interaction between fields in a highly specific manner, giving primacy to actors on either side of the boundary and dividing their roles in various ways.

Structural patterns organise research-policy relations in a way that puts primacy on the side of either research or policy. An array of models has been described in the literature in which either science or politics has relative primacy. For example, Weber (Weber, Owen, Strong \& Livingstone 2004) was very clear on the political primacy in science-policy relations. The task of the expert - generating knowledge - is distinctly separated from the task of the politician - deciding what to do with knowledge. Political decision-making involves choices in terms of values, goals and needs, which cannot be left to the rationality of the expert. Rather, science is drawn into the political administration dichotomy as a service of the rational development of policies based on politically set goals and values; it is politics 'on top' and science 'on tap'. This model has been described as the decisionistic model, as science helps politics make decisions. 
Habermas (in Outhwaite 1996) has a very different conception of the relationship between science and policy, which is closer to the traditional model of 'science speaking truth to power'. He claims that, in modern society, this relationship is often structured in such a way that the rationality of the expert dominates political decision-making and reduces value choices and goal-setting to technical and rational issues. This has been described as the technocratic model. In a technocracy, the politician 'becomes the mere agent of a scientific intelligentsia, which [...] elaborates the objective implications and requirements of available techniques and resources as well as of optimal strategies and rules of control,' leaving the politician 'with nothing but a fictitious decision-making power' (ibid.). According to Habermas, science has thus taken over relative primacy from politics.

Besides distributing relative primacy through their structure, boundary configurations have intrigue a number of sociologists, scientists and policy scientists for another reason. The structure of research-policy boundaries also involves specific rules concerning mutual relations - 'conditions of exchange' (Halffman 2003: 64). These conditions are not separate from the distribution of primacy, though they can be analytically distinguished. According to Wittrock (1991: 336), they are inherently connected to how research and policy are demarcated in patterns of boundary work: 'Any conceptualisation of [the research-policy] relationship ultimately rests on an assumption about the analogy between the operational modes of the realms of research and of policy'. Thus, depending on their demarcation, boundary configurations can involve rules of the game that establish a sharp division of labour between research and policy, or rules of the game that bring about more convergence in the roles of research and policy. Therefore, the rules structure either a divergence or convergence between the roles.

This focus on how the demarcation of research-policy relations are institutionalised in the rules of the game extends to the models of boundary configurations developed by Weber and Habermas. Weber's decisionistic or 'bureaucratic' model not only assumes political primacy, but also assumes clear-cut rules concerning the division of labour between research and policy. In this model, research would be drawn into the fact-value dichotomy that is also applied in the relationship between administration and politics, with science in the role of producing the facts and politics in the role of determining political values. In Habermas' technocratic model, the operational modes of science and politics are assumed to be more analogous or diffusely demarcated; science not only deals with the facts but also with values and, as such, it determines the political decision-making process. This means that in their theoretical conceptualisation of boundary configurations, the bureaucratic and technocratic models seem to be opposites in terms of the distribution of relative primacy, as well as the structural conditions of exchange. 
In addition to Habermas' technocratic model (scientific primacy, convergence) and Weber's bureaucratic model (political primacy, divergence), two other models of boundary configurations can be noted as possible theoretical combinations of primacy distribution and conditions of exchange. Wittrock (1991) and Hoppe (2005) have further distinguished an enlightenment model (scientific primacy, divergence) and an engineering model (political primacy, convergence) to offer other logical possibilities, which are depicted in figure 1.

Figure 1 Theoretical models of boundary configurations, based on Wittrock (1991) and Hoppe (2005)

\begin{tabular}{llll}
\hline & \multicolumn{2}{c}{ Coordination of field relations } \\
\cline { 3 - 4 } & & Scientific primacy & Political primacy \\
\hline $\begin{array}{l}\text { Demarcation } \\
\text { offield } \\
\text { structures }\end{array}$ & Divergence & Enlightenment model & Bureaucratic model \\
\hline
\end{tabular}

\section{Enlightenment}

In the enlightenment model, the research-policy nexus is structured in a way that establishes scientific primacy and sees divergence between the roles of scientific research and policy. It closely resembles the standard model of science, in which science is considered 'exceptional' because of its objective norms and methods. It contains a modernist hope that science will 'enlighten' supposedly irrational politics and policies. Science is to be as autonomous as possible, and policy development should be rationalised through its reliance on scientific evidence instead of political argument.

In this model, the sharp demarcation of science and policy also implies an absence of any pronounced institutional relationships. This means, on the one hand, an absence of policy interference in the field of research; the boundary between research and policy is heavily protected so as to maintain scientific autonomy. On the other hand, research is not directly involved in the field of policymaking; rather, the pervasive rationalisation of science will be largely indirect, through what Weiss $(1977,1991)$ has called a gradual 'knowledge creep'. That is, scientific knowledge finding its way into all facets of society. Scientific advisors, in this respect, are meant to speed up the diffusion process in the direction of government and politics.

These conditions of exchange in favour of protecting scientific autonomy are strongly connected to the distribution of primacy. The focus on autonomy reveals a value orientation that contains a fervent belief in scientific rationality and progress. Science is expected to deliver the conceptual and analytical tools as well as the technologies that steer policy and politics so as to, in turn, 'tame' the irrationality of politics. Thus, the rules 
concerning a sharp division of labour are clearly related to the idea of an enlightenment based on scientific research that is credible and independent.

\section{Technocracy}

As with the enlightenment model, science also enjoys relative primacy in the technocratic model, which posits that government and politics should be rationalised by science. However, the conditions of exchange between research and policy are more lenient in the technocratic model, creating a setting of convergence between the two domains. Technocracy involves scientists playing a direct role in policy development by virtually (possibly even literally) taking over the tasks of policymakers and politicians. In this respect, the technocratic model not only asserts that science 'speaks the truth', mirroring the enlightenment model, but also that science actually 'speaks truth to power' (Wildavsky 1979). For instance, scientific policy advice becomes more than a mechanism to speed up 'enlightenment'; it is also a mechanism for scientists to be more directly involved in policy design.

In this model, the research-policy nexus not only protects scientific autonomy (for speaking the truth), but also encourages involvement of science in the practice of policymaking and political decision-making. Rather than remaining in its ivory tower, science is meant to come down and translate its knowledge into policy practice. The development of a markedly institutionalised research-policy nexus can be an important manifestation of such structural convergence. Furthermore, this structural convergence is aimed at establishing scientific primacy, as technocracy contains a strong belief in the development of rational policies based on scientific rather than political involvement.

\section{Bureaucracy}

In the bureaucratic model, policy - or rather politics - retains its primacy. It is politics that decides what values and goals are set and how they are to be pursued in government policies. The bureaucratic model consists of clear-cut rules concerning the division of labour between scientific research and policy, defining the role of research as the production of facts and data as input for the political decision-making process. This way of creating divergence between the roles of research and policy is also meant to safeguard against a technocratic reduction of value choices to mere technicalscientific resolutions; politics should have primacy in dealing with normative issues.

A strong emphasis on political primacy in the bureaucratic model is compelled by the fact-value dichotomy that rules the exchange between research and policy. Another structural condition for exchange between research and policy in this model is that scientific research will often be drawn into the government administrative apparatus. On the one hand, this 
means that a firm institutional nexus between research and policy is created, for instance, in the form of a system of advisory bodies and planning offices closely associated with, or even part of, government. On the other hand, the fact-value dichotomy will remain an important condition for exchange between research and policy; instrumental research in this model is necessarily drawn into the politics-administration dichotomy. As with public administration, scientific research has to be responsive to the primacy of politics and refrain from normative involvement in policymaking, which is primarily the responsibility of politics.

\section{Engineering}

Finally, the engineering model also involves political primacy, allthough it defines the roles of research and policy in a more convergent way. As in the bureaucratic model, here it is 'politics on top and science on tap'. However, in the engineering model the fact-value dichotomy does not offer the possibility for exchange between research and policy. Rather, scientific research can be involved in the rational design of policies and the rational resolution of policy problems in a way that will also involve inherently normative facets. 'Engineering' is an apt name for the model because science becomes more closely involved in the rational engineering of society based on political priorities (in contrast to the technocratic model, where society is structured according to scientific beliefs).

Whereas the conditions for exchange in the engineering model involve more convergence between the roles of research and policy than in the bureaucratic model, the nexus between research and policy will be less institutionalised. In this case, the institutional relations between government or politics and scientific recommendations can be described as a principleagent relationship, in which the principle decides and the agent is deployed in service of the principle. Salter and Levy (1998) refer to this type of scientific research, which is produced in this model of boundary configuration, as 'mandated science'. Here, politics selects or commissions those models of scientific expertise that are considered useful. In the engineering model, research often acquires a prominent role in social engineering; however, the relations between research and policy tend to be provisional. Depending on the prevailing political values and goals, different sources of expertise can be mobilised; therefore politics as a principle can, at any time, change its dealings with specific agents.

\subsection{Research-policy dialogues and critical frame reflection}

Boundary configurations have various effects on the framing of issues like immigrant integration. They can either sustain or challenge the structural monopolies or 'iron triangles' within both fields (Heclo 1978), for instance, 
perpetuating a specific policy or research paradigm. They can also help create the structural conditions within research and policy that allow particular frames to emerge. However, research-policy dialogues do not necessarily involve the critical frame reflection that, according to Rein and Schön (1994), is required for resolving intractable controversies such as immigrant integration. In fact, the literature is rather sceptical about possibilities for reflection on frames in general, particularly about frame reflection in dialogues between research and policy. This book has thus chosen to examine, from a structuralist-constructivist perspective, how structures of the research-policy nexus have influenced frameshifts and problem framing in research and policy, and to what extent this involved frame reflection.

\subsubsection{Intractable controversies, frameshifts and frame reflection}

Rein and Schön (1994: 4, 1996: 240) refer to 'intractable controversies' as being those situations that are characterised by a multiplicity of frames or 'multiple social realities'. Intractable controversies involve 'frame conflicts', or 'struggles over the naming and framing of a policy situation [...] symbolic contests over the social meaning of an issue domain, where meaning implies not only what is at issue but what is to be done' (Rein \& Schön 1994: 29). Such frame conflicts differ fundamentally from disagreements about more structured problems, or problems that are characterised by a general agreement about 'problem framing' (Hisschemöller \& Hoppe 1995). They defy resolution by merely studying 'the facts', because actors with their own unique frames tend to select different sorts of factual evidence and, even if they agree on a selection, tend to interpret it differently. For instance, the relevance of factual evidence on the educational achievements of migrants depends on the relevance of education as a sphere of integration in a specific frame. In addition, evidence about educational achievements can still be interpreted in many ways, for example, as an indication of migrants' progress over time or as an indication of persisting relative deprivation in comparison to other social categories.

Frameshifts, then, involve fundamental changes in how actors define and understand problem situations. They constitute 'reality shifts' (Fischer 2003: 155), rather than merely different ways of perceiving a problem. Social science literature is brimming with references to the difficulties of achieving such frameshifts. For instance, both Hall and Sabatier question the capacity of individuals to become aware, reflect on or even alter their most fundamental beliefs, as this would be similar to religious conversion. They believe that 'learning' on a cognitive level could only lead to relatively minor adjustments of frames (Hall 1993; Sabatier 1987; Sabatier \& Jenkins-Smith 1999: 123). Frameshifts, rather, would be the consequence of changes in the institutional context or, as Sabatier describes them, 
'external perturbations' (Sabatier \& Jenkins-Smith 1999: 118), involving broader cultural changes ('mood swings'), social-economic developments, political shifts and constitutional changes. These institutional changes would lead to frameshifts by changing the power relations (distribution of resources, capital) between actors with different frames, rather than bringing about mere awareness of different frames. Agenda-setting theory is also rather sceptical of opportunities for actors to become aware of their tacit frames. It hypothesises frameshifts as the consequence of agenda-setting, something which, according to Baumgartner and Jones (2002: 15-23), is achieved primarily by shifting attention to other problem facets, rather than by reflecting on a frame (attention shifts or non-contradictory argumentation) or through the social process of mimicking (unreflectively adopting the ideas of others). Agenda-setting theory also refers to the relevance of the aforementioned 'external perturbations', such as the occurrence of focus events (Kingdon 1995: 94).

In contrast, Rein and Schön (1994: 37) believe that actors can become aware of their own frames and critically reflect on them. This calls for critical reflection on a frame's internal consistence and coherence, as well as on its relationship to developments in a problem situation and to society at large. Actors therefore would be able to reflect critically on the extent to which their frame offers a convincing story about a problem situation, whether it fits the evidence and whether it gels with the broader normative perspective.

Starting with the analysis of controversy as frame conflict, we propose that human beings can reflect on and learn about the game of policymaking even as they play it, and, more specifically, that they are capable of reflecting in action on the frame conflicts that underlie controversies and account for their intractability. In our view, human beings are capable of exploring how their own actions may exacerbate contention, contribute to stalemate, and trigger extreme pendulum swings, or, on the contrary, how their actions might help to resolve the frame conflicts that underlie stubborn policy disputes. We believe that hope for human reason in the chaotic, conflictual world of policy-making lies in a view of policy rationality that gives a central place to this human capability for reflection 'within the game'. (ibid.: 37-38)

Frame reflection is, according to Rein and Schön, always 'situated'. This means that there is no universal law for how frame reflection can be achieved. How and to what extent it can be achieved will depend on the institutional setting (situational context). Thus, frame reflection could lead to the 'situated resolution of frame controversies' (ibid.: 176). Such a belief marks the difference between relativist approaches that discard the 
possibility of frame reflection and objectivist approaches that see framing as a consequence of structural developments rather than reflection. It also deviates from Habermas' (in Outhwaite 1996) perspective on how to achieve reflection beyond relativism and objectivism by establishing a socalled ideal-free speech situation in which a power-free social context is created in order to reflect on frames. Indeed, Rein and Schön believe that frame reflection must be achieved within the structural setting of a problem situation, rather than by creating a setting that is removed from the actual structural setting of the actors involved.

\subsubsection{Boundary configurations and frameshifts}

Boundary configurations can contribute to frameshifts through their effect on the field structures of social science research and policymaking. These field structures provide the structural setting in which problems are framed. Boundary configurations can contribute to change by reinforcing either the pressure to enforce stability or the pressure to enforce change. Baumgartner and Jones (1993: 19) have referred to situations in which field structures manage to reproduce themselves as 'structure-induced equilibria'. They emphasise how these equilibria are 'structure-induced' in that they are consequences of historically developed structures that resist change. This can involve lock-in effects of past developments or path dependency (Pierson 1994), as well as the structural distribution of specific species capital that generates interests for at least some of the actors in preserving the prevailing structure (Baumgartner \& Jones 1993: 19; Bourdieu \& Wacquant 1992: 105). For instance, once a particular boundary configuration is institutionalised and has created subsequent boundary organisations, the organisations will have an interest in preserving this configuration in order to maintain their structural position.

By reinforcing such structure-induced equilibria, boundary configurations can generate 'negative feedback' in relation to the ongoing dynamics in field structures (Baumgartner \& Jones 1993: 16). This means that the ongoing dynamics is affected in a way that inhibits change and induces stability. For example, this may occur when research-policy relations give legitimacy to established policy institutes or when policy institutes fund established research institutes. However, boundary configurations can also influence the dynamics in a field by mobilising 'positive feedback' (ibid.). Positive feedback means that ongoing dynamics are affected in a way that promotes change and challenges the status quo. There are no universal laws for how and when the research-policy nexus could or should contribute to negative or positive feedback. Its role in field dynamics is highly dependent on the structural setting of ongoing dynamics in the fields and, of course, on the structure of the research-policy nexus. 
In structuralist-constructivist as well as institutionalist literature, it has been observed that structural patterns of negative feedback in support of structure-induced equilibria tend to be occasionally interrupted by positive feedback and dramatic breakthroughs. Institutionalists have argued that once negative feedback mechanisms are disrupted, change will occur in a 'ruptured manner' that is 'episodic and dramatic, responding to institutional change at the macro level, rather than incremental and smooth' (Powell \& DiMaggio 1991: 10-11). This pattern of punctuated equilibrium with episodes of relative stability as well as ruptures of dramatic change has been described in terms of 'punctuations' (Baumgartner \& Jones 1993) or 'paradigm shifts' (Hall 1993). Although such a pattern has been observed primarily in studies of policy developments, it can reasonably be extended to the study of research developments, at least from a structuralist-constructivist perspective that defines both policy and research as fields of structured social relations.

By affecting the field structures of research and policy, boundary configurations will affect the structural setting in which problem framing takes place. As such, they not only contribute to the structural conditions for frameshifts, but also to the structural conditions for the emergence of specific problem frames. As Schattschneider (1960: 71-73) has observed, every structure or every form of organisation necessitates a 'mobilisation of bias'. Thus, any way of changing a field's structure also involves a change in this selective mobilisation of bias.

Baumgartner and Jones further developed this idea of selective mobilisation of bias in their conception of how problem images interact with particular institutional venues. Their central premise is that some structural settings are more susceptible to some frames than others. As such, actors will seek those structural settings that are most receptive to their frames. This has been described in the literature as 'venue shopping' (Baumgartner, Green-Pedersen \& Jones 2006; Baumgartner \& Jones 1993; Guiraudon 2000a; Pralle 2003). Thus, venue shopping can motivate actors in either research or policy to seek access to the other field when the field structure is considered more receptive to a specific problem frame. For instance, researchers who are relatively marginalised in the scientific field can seek support from policymakers if they think their ideas match those of particular actors in the policy field. Moreover, actors can try to change the structure of their fields so that it becomes more receptive to their frames. However, as observed earlier, such structural changes are also often achieved through interaction with other fields, rather than as a consequence of internal changes. In both respects, boundary configurations can play an important role in searching for structural settings that are receptive to specific frames.

Although, again, there is no universal law for how and why structural settings promote problem framing, there have been numerous studies about 
how different structural boundary configurations can affect problem framing. Nelkin (1979) observed how structural settings with deep policy involvement by researchers, or even those that limit participation to a network of researchers, tend to create technical-scientific problem frames. This would have occurred notably in the domain of environmental policies, where technical matters often dominate regulatory policies, thus obscuring ethical aspects of the environment.

Regarding migration and immigrant integration, Guiraudon (1997) has drawn attention to a correlation between the scale of the debate on these issues and the extent to which frames were adopted that supported the extension of migrant rights. Experts and research institutes often limited the scale of debate, which - thanks to the lack of much mass media or political attention - facilitated the extension of migrant rights. The same would apply for administrative bodies and legal venues that limited participation. Guiraudon (2000a) also showed how national governments 'shopped' for intergovernmental venues on a European level, seeking to get their ideas on restrictive immigration policies accepted beyond the influence of national venues that might have opposed such policies.

\subsubsection{Frame reflection}

Boundary configurations may contribute to frameshifts and problem framing, but this does not necessarily involve frame reflection. Rein and Schön (1994: 37) believe that actors are able to reflect on their usually tacit frames in actual social practices. They believe that 'hope for human reason in the chaotic, conflicting world of policy-making lies in a view of policy rationality that gives a central place to this human capability to reflection "within the game" (ibid.: 338). While they focus primarily on reflection in policy practices, Bourdieu (2004) looks more to reflexivity in the scientific enterprise. This refers to 'the systematic exploration of the unthought categories of thought which delimit the thinkable and predetermine the thought as well as guide the practically carrying out of the social inquiry' (Bourdieu \& Wacquant 1992: 40). Along with Rein and Schön, Bourdieu refers to reflecting on structures or institutions as well as reflecting on the framing of reality, or as Bourdieu and Wacquant (ibid.) describe it in the context of the scientific field: 'the collective scientific unconsciousness embedded in theories, problems and (especially national) categories of scholarly judgement'.

From a structuralist-constructivist perspective, these scholars have discussed the structural conditions for achieving reflectivity. In scientific studies, Bourdieu has pointed to how structural dependencies between the scientific field (or the academic/intellectual field) and developments in other fields have obstructed reflexivity or the progress of scientific reason. Throughout his work, there has been expression of a particular concern 
regarding the autonomy of the scientific field. Within well-articulated borders, emphasising control over entry and the logic of the scientific field, Bourdieu (2004: 54) argues as follows:

The fact that producers tend to have as their clients only their most rigorous and vigorous competitors, the most competent and the most critical, those therefore inclined and most able to give their critique full force, is for me the Archimedean point on which one can stand to give a scientific account of scientific reason, to rescue scientific reason from relativist reduction and explain how science can constantly progress towards more rationality without having to appeal to some kind of founding miracle.

Rein and Schön (1994: 176) have given further thought to how to achieve such critical reflection. They argue that frame reflection could lead to what they call the 'situated resolution of frame controversies. Frame reflection would thus most likely be achieved within a situational setting characterised by 'design rationality' (Rein \& Schön 1994: 166-187). Several structural conditions can be derived from their discussion of design rationality, that would lead to critical frame reflection.

Firstly, Rein and Schön (ibid.: 182) posit a communicative imperative. This means that actors involved in a disagreement must be willing and able to communicate openly with one another, and should not exclude specific actors from the frame conversation. Frame reflection must therefore be an open social process. Secondly, actors must be able to identify alternative frames and try to understand how actors within such frames make sense of problem situations (ibid.: 176). This means that they must be capable of empathy. Thirdly, actors must become aware of their own frames, possibly in interaction with the identification of alternative frames (ibid.: 174). This creates the possibility to reflect critically on one's own frame and to search for possible design flaws, such as internal inconsistencies or incoherencies within that frame. The same applies to incompatibilities with new information or knowledge of problem developments that may contradict a frame. Fourthly, actors must not only reflect on their own frames and alternative frames, but also be willing and able to act when design flaws are traced or when the confrontation with alternative frames produces undesirable results (ibid.: 186). This requires a certain pragmatism - not in terms of the framing itself but of an unbiased willingness to adapt one's frame if necessary. Finally, frame reflection usually requires mutual trust (ibid.: 179). Without trust, necessary capacities such as communication, empathy, being able to correct one's own frames and pragmatism are unlikely to thrive.

In sum, boundary configurations can contribute to frame reflection by satisfying these five structural conditions - openness, empathy to alternatives, critical reflection, pragmatism and trust. This means that to achieve 
critical research-policy dialogues, research must extend its role beyond that of the traditional model of 'speaking truth to power', in which researchers dictate how problems are framed. In addition, politics must move beyond the positivist belief in scientific truth as well as the relativist predicament that science is nothing but politics by other means. Rather, to achieve frame reflection, scientific research should help politics make sense of the issues (Hoppe 1999; Rein \& Schön 1996). Instead of producing knowledge that is robust in its scientific methodological foundation, research has to produce 'socially robust knowledge' that is founded on critical debates about problem framing (Nowotny et al. 2001: 166).

\subsection{Research design}

As already stated, this book takes an empiricist approach to research-policy relations and problem framing. It empirically studies boundary work and boundary configurations rather than doing boundary work and constructing boundary configurations. This epistemology emanates from the structuralist-constructivist perspective, which focuses on how actors make sense of research-policy relations in actual social practices and how they make sense of problem situations within specific structural settings of social relations.

This book avoids an ex ante theoretical position concerning the framing of immigrant integration. It takes a step back from the ongoing controversies over the framing of this issue to analyse instead controversies over problem framing. It is not the framing of immigrant integration that is the subject at hand but rather, the structural settings in which this framing process takes place. Avoided here as well is an ex ante theoretical position concerning the configuration of research-policy relations. Instead, this book will provide an empirical reconstruction of how actors produce and reproduce boundary configurations in their actual social practices. Boundary work is studied empirically by analysing that which is carried out by 'realworld' actors (Gieryn 1995: 394). Philosophical arguments will not be given in favour of one model of research-policy relations, but provided instead are empirical arguments on how and why actors came to relate in specific ways and what empirical effects this has had in terms of problem framing.

This study thus seeks to contribute to reflection on the part of those actors actually involved in research-policy relations and problem framing in this domain. With an empirical analysis of boundary work and problem framing in actual social practices, it aims to raise awareness about these social processes among actors in the domain. Through such awareness, an empirical reconstruction could contribute to learning how to structure 
science-policy relations in order to promote critical dialogues between research and policy at the level of problem framing.

\section{Research questions}

This book's fundamental question is thus: what part did the research-policy nexus have in research and policy frameshifts concerning immigrant integration in the Netherlands over the past decades? Logically following, how can this be explained? And to what extent did the research-policy nexus contribute to critical frame reflection? This formulation positions this study as an enquiry into the role of the research-policy nexus in problem framing, rather than a study of immigrant integration research, immigrant integration policy or, for that matter, a study of immigrant integration per se.

The research question will be further elaborated based on the structuralistconstructivist perspective and theoretical concepts discussed in this chapter. Chapter 3 focuses on what frames have emerged and what frameshifts have taken place in Dutch immigrant integration research and policy over the past decades. This research builds on other studies that have shown how the framing of immigrant integration has changed significantly over the past decades (Entzinger 2005; Snel \& Scholten 2005). Such frameshifts are generally understood to be an indication of the intractability of a topic. Thus, the intractability of immigrant integration is considered a starting point (rather than an empirical outcome) and, as will be discussed later, one of the core reasons for formulating this research question and selecting this case study. This chapter will provide a reconstruction of the intractability of immigrant integration by identifying the frameshifts that have taken place over the past decades. It is of great importance for the study, as it selects the research-policy frameshifts that will be analysed in more detail.

Subsequently, in chapters 4, 5 and 6, a number of questions will be raised further concerning the relations between research and policy in the three main frameshifts to have taken place in Dutch immigrant integration policies over the past decades. This requires, firstly, an analysis of what kind of research and which policy actors were involved, what were their frames and what positions did they hold in the fields of research or policy. The emphasis here is on those actors involved in the frameshifts to be identified in response to the first question. It involves an analysis of the context in which this frameshift took place so that relevant research and policy actors can be identified. A reconstruction of the actors' frames will consequently follow. Discussions concerning actors and context will be entwined because, from a structuralist-constructivist perspective, the role of context is considered to be mediated through actor frames and the positions that actors hold within their research or policy fields. For every period that a frameshift is identified, this second question will depict the actor setting and the contextual setting. Within this playing field, more questions will 
focus in greater detail on the research-policy nexus and its role in these frameshifts.

Secondly, an analysis will be made of how these actors define the relationship between research and policy and how this can be explained. This question is likely to produce different answers depending on the periods in which the frameshifts took place. It draws attention away from problem framing and towards how the actors defined the relationship between research and policy, thus clearly adopting an empiricist approach to the analysis. Furthermore, informed by the structuralist-constructivist perspective, it asks how actors' positions within the field structures of immigrant integration research and policy may provide an explanation for how they defined research-policy relations.

Thirdly, this book will solicit what structural research-policy configurations can be identified. This will also produce answers that vary according to the different frameshifts. Here, attention is transferred from actors to the structural setting of research-policy relations. There is also an attempt to identify the more structural boundary configuration that was produced and reproduced by actors from both fields.

Finally, an analysis is made of the role these boundary configurations have had in frameshifts in research and policy. Specially, what has been their role in problem framing and/or reframing, and to what extent did their role involve critical frame reflection? These enquiries call attention to the effect of boundary configurations on the structural settings of immigrant integration research and policy and their role in promoting either change or stability. Furthermore, they raise the issue of how the structural effects of boundary configurations may have contributed to the rise or fall of specific problem frames. The two sub-questions address, from a structuralist-constructivist perspective, what has been called both the structure and the culture of public problems. The final element of this question involves the extent to which this effect on problem framing involved frame reflection, or whether reframing was (as predicted in the literature) a consequence of factors other than reflection.

Following these case chapters, in chapter 7 a comparison will be made of findings from the Dutch case and those from Germany, France and the UK. The aim of this comparison is to find out whether there are more general patterns in research-policy dialogues or whether the Dutch case is, in fact, characterised by exceptionalism.

\section{Case study design}

This book adopts an 'embedded, single-case study design' (Yin 1994: 42). It is a single-case study because it analyses the role of the research-policy nexus in problem framing in one case: the issue of immigrant integration in the Netherlands over the past four decades. It is 'embedded' because, although it involves one object of analysis - actors involved in research- 
policy relations in the domain of immigrant integration - it involves two embedded units of analysis: research-policy relations and immigrant integration policy frames.

A strength of this design, as it is applied here, is that it allows for an indepth look at the role of the research-policy nexus in problem framing with full appreciation of the situational setting where this nexus is constructed and problem framing takes place. An empirical epistemology requires that boundary work and problem framing be studied within their 'real-life context' (ibid.: 14). Many variables will result and the requirement of gathering as much data as possible on as few variables as possible (King, Keohane \& Verba 1994) cannot be met. The research-policy nexus and problem framing cannot be defined beforehand in a way that will create clear-cut 'boundaries between phenomenon and context' (Yin 1994: 14). This is because, from the perspectives of framing and boundary work, the construction of what passes as context is considered endogenous to these social practices (ibid.). According to Yin (ibid.), this is one situation in which research would best be served by adopting a case study design. Therefore, an in-depth, single-case study is the most suitable for gathering sufficient data (for instance, for triangulation) within the scope of this research and in order to be able to say anything meaningful about boundary work in research-policy relations and its correlation to problem framing.

It is important to observe that single-case studies do have limitations in terms of opportunities for generalisation. They provide in-depth knowledge of the specifics of one case, without much analytical leverage in terms of explaining other cases; their external validity is inherently limited. While there is potential for analytical generalisation and theory-building, a singlecase study does not lend itself to empirical generalisation in the context of research-policy dialogues on migration and integration in other countries, or in other issue domains. As such, this research design contains a comparative analysis in which findings from the Dutch single-case study are compared with available evidence on research-policy dialogues on migration in other European countries. This comparison involves so-called pattern matching: examining whether the configurations of research-policy dialogues that have been uncovered in the Netherlands can also be found in the other countries. For instance, this study finds how a technocratic research-policy configuration contributed to the rise of multiculturalism in the Netherlands in the early 1980s. Through comparison, one aim would be to discover whether such relations in other countries have also been configured in a technocratic manner and, if so, whether this similarly contributed to the rise of multiculturalism. Although a full comparison of research-policy dialogues between all countries is not made, this study does support extending empirical generalisation from the Dutch case to the others. 
The countries for international comparison were selected according to renowned differences in research traditions in the field of immigrant integration. In sharp contrast to the Netherlands, France has been known for its sensitivities surrounding social-scientific research on immigrant integration (the taboo on statistiques ethniques), as well as for the deep political engagement of researchers with, often, a political or philosophical background. In Germany, despite its persistent denial of being a country of immigration, social researchers have concentrated primarily on social-economic issues like education and labour market participation, rather than the more political concerns with cultural assimilation dominant in France. The UK has been characterised by a much more institutionalised research-policy nexus in which sociologists and anthropologists were heavily involved in a more colour-oriented British approach. This book seeks to discover whether these unique ways of organising research-policy dialogues have also led to the construction of different frames.

The combination of an in-depth study of the Dutch case and pattern matches between several other cases provides a sound basis for analytical generalisation. Analytical generalisation concerns generalisation of theoretical propositions, using techniques for developing 'grounded theory' (Glaser 1992) - which need not be exclusive to the field of immigrant integration. More generally, this book will offer insights into how research-policy relations are configured and how research can contribute to critical frame reflection on intractable policy controversies. In this respect, immigrant integration represents a 'showcase' or 'revelatory case study' (ibid.: 40); the framing of immigrant integration as well as the structure of research-policy relations in this domain have become fiercely contested in the Netherlands in recent decades. As such, this book strategically provides a window through which to study the role of the research-policy nexus in problem framing.

\section{Building a valid and reliable chain of evidence}

Central to a sound research design is constructing a valid and reliable chain of evidence involving the key concepts and theoretical relationships. The first step in this chain of questions in chapter 3 is to uncover the extent to which immigrant integration has been an intractable topic in research and policy. This means looking for changes in problem framing in research and policy. In order to ensure validity, the frame concept has been operationalised into four attributes: terminology, social classification, causal theories and normative perspectives. The search for indicators of frameshifts involves an analysis of policy documents and secondary literature on immigrant integration research and policy developments in the Netherlands over the past decades. For the policy field, policy memoranda constituted a valid and reliable source of problem framing. Changes in policy contours are the main reason why a new policy memorandum is issued in the first place. 
Such key texts are not available for the scientific research field. Abundant secondary literature about changing perspectives on immigrant integration was therefore examined for this study, in order to create a reconstruction of changes in terminology, social classification, causal theories and normative perspectives, so as to ensure the validity of the use of the frame concept in this respect.

The second step in building a chain of evidence is to zoom in on the objects of analysis - the actors involved in the research-policy nexus. Actors who played an important role in research and policy developments (e.g. experts, research institutes, advisory bodies, policy departments, political institutes) naturally turn up in the literature describing the above-mentioned first step. Emphasis thus is on the frames of the identified actors and their reconstructions of the contextual setting of problem framing. The frames will be studied by scrutinising the documents they produced (e.g. research reports, advisory reports, government documents). Furthermore, the contextual setting in which these frames emerged will be analysed by reconstructing their positions within the research or policy fields, specifically by looking for background knowledge on their positions (formal positions and roles, resources and informal relations and networks) and the rules of the game within both fields (social norms of doing science or making policies, methodological paradigms, etc.). Furthermore, these developments are placed in the context of macro-institutional developments in scientific research and policymaking.

The third step focuses not on these actors' frames, but on their social practices and positions within the broader fields of research and policy. Analysed here is the boundary work of these actors by studying their 'literary, social and material technologies' (Shapin \& Schaffer 1985), also known as 'boundary discourse', 'boundary relations' and 'boundary objects' (Halffman 2003: 63). Interviewing constituted the primary method in this regard. The interviews were semi-structured, following a list of topics that was deduced from the central research questions. Triangulation was used as a methodological strategy for enhancing reliability, involving data triangulation (using multiple sources of data) as well as methodological triangulation (using multiple research methods) (Yin 1994: 90). Interviewing was combined with analysis of primary documents, such as minutes, records and notes that were kept by actors themselves (e.g. from the Scientific Council for Government Policy and the Department of Home Affairs, as well as records of parliamentary hearings) and secondary sources like media records and scientific literature.

The fourth step in building a chain of evidence shifts attention from the actors to the structural setting of research-policy relations or boundary configurations. Indicators of such configurations can be found in the patterns of interaction between research and policy actors. Indicators of how relative primacy was configured can be found in instances where either the 
research actors determined policy developments or policy actors determined research developments. Indicators of convergence or divergence can be found in the extent to which research and policy interacted either directly (close mutual relations) or more indirectly (at a distance). These indicators will be studied using the same methods and sources employed for the analysis of boundary work practices in the third step.

The fifth step of this research design connects evidence from the structural setting of research-policy relations to that in which the frameshifts took place. Firstly, this requires an examination of how the research-policy nexus strengthened the position (in terms of legitimacy, authority and resources - or 'capital' in general) of specific research and policy actors who advocated either change or stability in terms of problem framing. Indications of such effects can be found in references to how the researchpolicy nexus provided capital to specific actors, both within interviews with these actors and within secondary literature about frameshifts. Secondly, the step involves an analysis of how boundary configurations played a role in the rise or fall of specific problem frames. An indication of such influence would be the exclusion of actors within specific frames from the field structures. This requires an examination not only of how the positions of actors are altered, but also how these changes affected actors within different frames. Finally, this step involves an analysis of the role of boundary configurations in frame reflection. In this regard, the data that was gathered for the role of boundary configurations in frameshifts and framing is discussed in relation to the aforementioned attributes of frame reflection - openness, empathy, critical reflection, pragmatism and trust.

Finally, the sixth step (in chapter 7) involves an international comparison, or more precisely, a pattern-matching between findings from the Dutch case and the available evidence on research-policy dialogues in Germany, France and the UK. This section is based primarily on an extensive review of the international literature on immigrant integration policy, research and their mutual relations in these countries. Although few studies have made the research-policy nexus in this domain the object of their analysis, there are a number of excellent studies that, to some extent, discuss either the development of immigrant integration research or the role of research in policymaking (Guiraudon 1997; Boswell 2009; Bommes \& Morawska 2004; Vasta \& Vaddamalay 2007; Favell 2001). The comparative analysis in this book has been shaped by a series of informal interviews with researchers and policymakers from the respective countries over the past years. These interviews helped provide access to relevant information on the countries and to find pertinent sources. An important occasion for this was the international gathering mentioned in the introduction, the Research-Policy Dialogues on Migration and Integration in Europe conference held in 2008 in Enschede, the Netherlands, which brought together policymakers and researchers from various countries. The 
sessions held during this special conference provided material that has been used throughout this book. Furthermore, the comparative chapter was distributed to, and discussed with, various country experts.

\subsection{Conclusion}

This book attempts to capture the dynamics of how immigrant integration has been framed and reframed in recent decades. It develops a structuralistconstructivist perspective to get beyond the predominant focus on 'national models of integration', thus honing in on the dynamic ways in which immigrant integration is framed. Each frame involves a different terminology, a different way of classifying involved groups or categories, different causal stories as well as a different normative perspective.

Revealed, too, are the structural settings in which problems are socially constructed or framed, specifically the configuration of the research-policy nexus. Both immigrant integration research and policy are analysed as distinct fields of structured social relations, each with unique positions, rules of the game and a distribution of capital. Problems are framed against the background of these structural settings. Field structures are produced and reproduced, using different ways of demarcating themselves from other fields and coordinating relationships with other fields. This boundary work affects the shaping of field structures and relations. As such, boundary work can also play a role in producing specific structural settings in which problems are framed in research and policy.

As this book shows, institutionalised patterns of boundary work can create structural configurations of research-policy relations. Boundary organisations have a structure of their own, coordinating relations between both fields in specific ways and also demarcating the roles of both fields with specific rules of the game. To reiterate, four theoretic models of boundary configurations are distinguished: enlightenment, technocracy, engineering and bureaucracy.

Boundary configurations form part of the structural settings in which problems are framed. They can either reinforce the structural dynamics towards change (positive feedback) or reinforce prevailing structure-induced equilibria (negative feedback) within the fields of scientific research and policymaking. As such, they can stimulate and inhibit frameshifts. In addition, boundary configurations may contribute more specifically to the rise or fall of particular frames. Various studies have revealed a correlation between the involved venues in problem framing and the type of frames that emerge. However, the role of boundary configurations in problem framing does not necessarily involve frame reflection. According to extant literature, frame reflection will only occur when the structural setting promotes openness, empathy, critical reflection, pragmatism and trust. Boundary 
configurations only contribute to the situational resolution of intractable controversies through frame reflection when they contribute to these specific structural conditions.

This book adopts an empiricist methodological approach to answer its central question: what has been the role of the research-policy nexus in the frameshifts in immigrant integration in research and policy in the Netherlands over the past decades? Moreover, how can this role be explained and to what extent did the research-policy nexus contribute to critical frame reflection? This approach is founded on an empirical epistemology that studies the research-policy nexus and frames of immigrant integration by examining how actors undertake boundary work and frame problems in actual social practices. Rather than carrying out boundary work, the boundary work is studied; and rather than carrying out problem framing, problem framing is the object of study.

Though a single-case study, this book attempts to achieve analytical generalisation. The bottom line is that the research-policy nexus does play a role in problem framing. This study aims to provide theoretical insights into how the research-policy nexus can be structured to promote critical frame reflection for the situated resolution of such controversies. Furthermore, the international comparison provided aims to strengthen the external validity of this research by matching the patterns of researchpolicy relations found in the Dutch case to those in others. In this way, we can answer a burning question: is the Dutch case is 'exceptional'? Or, can more general hypotheses be developed on the relation between specific types of the research-policy nexus and the rise and fall of specific frames of immigrant integration. 


\section{Frames and frameshifts in Dutch immigrant integration policy and research}

The Netherlands is often seen as being representative of the so-called multicultural model of integration. Historically, the Dutch have had a tendency to recognise cultural groups in society and to emancipate them through structures that reflect the specifically Dutch history of 'pillarisation'. In fact, it is because of the alleged success of this multicultural model in accommodating cultural differences in society that scholars and policymakers from Europe have shown great interest in the Dutch case.

However, there has been surprisingly little empirical research into how this model of integration evolved or whether there are alternative models that may apply to the Netherlands. This creates great difficulties when it comes to debates about the alleged success or failure of this multicultural model. After the tumultuous year of 2002 in which Fortuyn placed immigrant integration at the top of the political agenda, the Dutch multicultural model was declared a failure. At the same time, there was sharp controversy about whether the Dutch case should be described as multicultural at all, especially as the 1990s had witnessed the rise of a more socialeconomic approach. Furthermore, others, including the Parliamentary Investigative Committee on the Integration Policy established in 2004, concluded that the emergent Dutch model had been rather successful, especially in terms of the progress made in social-economic areas such as education and labour. To complicate the situation further, the infamous 'Dutch multicultural model' appeared to have become a counter-discourse in public and political debate, one used to support an assimilationist turn in Dutch policies. In contrast to social-economic participation or social-cultural emancipation, social-cultural adaptation was now increasingly considered to be the core of a new 'Dutch approach'.

Clearly signified here is the multiplicity of frames that characterises immigrant integration in the Netherlands. This chapter will analyse that multiplicity, according to an empirical study of the development of immigrant integration policy and research in the Netherlands. It will reconstruct the problem frames that have emerged in research and policy and identify frameshifts by examining scientific literature and policy documents and by looking for changes in the different attributes and indicators of problem framing - terminology, social classification, causal stories and normative perspectives. 


\subsection{The Dutch multicultural model}

A key trait of the so-called Dutch multicultural model is its tendency to institutionalise cultural pluralism. This is based on the belief that the cultural emancipation of immigrant minorities is the key to their integration into Dutch society (see e.g. Duyvendak \& Scholten 2009). This also reflects a rather uncontested acceptance of the transformation of Dutch society into a multicultural society. In fact, a connection is often drawn to the peculiar Dutch history of pillarisation, referring to the period from the 1920s to the 1960s when most of society was structured according to specific pillars religious (Protestant, Catholic) and socio-cultural (socialist, liberal) (Lijphart 1968).

Sniderman and Hagendoorn's (2007) book When ways of life collide: Multiculturalism and its discontents in the Netherlands understands the Dutch approach in terms of a multiculturalist model. The authors claim that the labelling of collective identities has inadvertently deepened socialcultural cleavages in society, rather than bridging differences. They take the Netherlands as a token case to underpin their claims, rooting the Dutch approach in the history of pillarisation: 'The Netherlands has always been a country of minorities thanks to the power of religion to divide as well as unite' (Sniderman \& Hagendoorn 2007: 13). In addition, the 'collective trauma of World War II where the Dutch failed to resist the massive deportation of Jews' contributed to the fact that 'immigrant minorities have been seen in the light of the Holocaust [...] or that critical views of immigrants are labelled racist and xenophobic' (Sniderman \& Hagendoorn 2007: 15). Given these historical circumstances, a multiculturalist model would logically take root in the Netherlands.

The German sociologist Joppke also considers the Dutch the most radical exponents of the multiculturalist model. He describes how the Netherlands recently changed its policies as a consequence of (allegedly) multicultural policies being a failure.

Civic integration is a response to the obvious failure of one of Europe's most pronounced policies of multiculturalism to further the socioeconomic integration of immigrants and their offspring. [...] In a counterpoint to multiculturalism's tendency to lock migrant ethnics into their separate worlds, the goal of civic integration is migrants' participation in mainstream institutions. (Joppke 2007: 249)

The Dutch multicultural model has also found resonance among Dutch scholars. Koopmans $(2002,2003)$ roots the Dutch approach to immigrant integration clearly in the history of pillarisation, when ethno-cultural cleavages were stressed in a similar way in multicultural policies. He claims that the application of this model to new immigrant groups has had major 
adverse effects, as multiculturalism 'offers new ethnic and religious groups a formal and symbolic form of equality, which in practice reinforces ethnic cleavages and reproduces segregation on a distinctly unequal basis' (Koopmans 2002: 92). Koopmans points, in particular, to 'path dependency' in terms of policy practices. Although formal policy discourse and public discourse seem to have changed, the Dutch have remained accommodating in the way they deal with ethno-cultural diversity.

The Netherlands is still an extreme representative of a 'multicultural' vision of integration. [...] Outside the limited world of op-eds in high-brow newspapers, the relation between Dutch society and its immigrants is still firmly rooted in its tradition of pillarisation [...] [O]rganisations and activities based on ethnic grounds are still generously supported - directly and indirectly - by the government. Whether people want it or not, ethnicity still plays an important role in public institutions and discourse. (Koopmans 2002: 91; author's translation)

Rather than examining the Dutch multicultural model, per se, or evaluating its alleged success or failure, the objective of this book is to study the construction of this type of model. In other words, it is not about the model itself, it is about the model as a discourse. By asking why we have come to talk about the Dutch approach in terms of the multicultural model in the first place, this study takes a step back from current debates to critically understand the Dutch multicultural model.

\subsection{The rise and fall of policy frames}

Empirical analysis of the development of Dutch immigrant integration policies teaches us that the Dutch case should be described as a succession of frames, rather than something defined by a single dominant model, as is often suggested in national and international literature. Immigrant integration policy appears to have been marked by episodes of stability as well as periods of dramatic change (Entzinger 2005; Scholten \& Timmermans 2004). Various policy frames have come and gone over the past decades. This makes it difficult to speak of one immigrant integration policy. Rather, it seems that there have been several immigrant integration policies over time. An analysis of official policy documents shows that at least four policy episodes can be distinguished, each characterised by a particular dominant policy frame and separated by frameshifts: the lack of immigrant integration policy until about 1978, followed by a minorities policy until the early 1990s, an integration policy until the turn of the millennium and, more recently, the integration policy 'new style'. 


\subsubsection{The lack of immigrant integration policy}

Until the 1970s, no immigrant integration policy existed in the Netherlands. It was considered unnecessary, as immigrants were expected to eventually return to their home countries. The Moluccans, for example, were to one day return to either an independent Moluccan republic or to Indonesia itself, and foreign workers were seen as temporary 'guest workers', who would go back to their countries of origin when there was no longer a demand for supplementary labour in the Dutch economy. Two other major immigrant groups from former Dutch colonies - the Surinamese and the Antilleans - were not considered permanent immigrants either, because they were seen as fellow citizens of the Dutch Kingdom who could enter and leave the Netherlands as they pleased. In addition, there was no common framework for policies related to the country's various immigrant groups. Policies on foreign workers fell under the responsibility of the Department of Social Affairs; asylum migration was coordinated by the Department of Foreign Affairs; and policies regarding the Surinamese and the Moluccans came under the remit of the Ministry of Culture, Recreation and Social Work (from herein referred to as CRM).

On the whole, the policies that were developed for these migrant groups appear to correspond to the differentialist model. In fact, the so-called 'two-tracks' policies (Choenni 2000) developed in this period implied that, though migrants were to be activated in the social-economic sphere, in other respects they were differentiated from Dutch society. This differentialist frame is primarily manifested in policy and political discourse, under the slogan 'Integration with retention of identity'. Initially, this did not carry the permanent connotation that it would acquire later, but rather referred to the social and economic integration of migrants during their stay in the Netherlands. This meant that the social and economic well-being of migrants had to be assured for the length of their stay, which had an additional purpose of maximising their economic participation. Policy towards foreign workers was:

aimed at, given the existing and expected needs of our economy to deploy foreign labour, balancing as much as possible the number of foreigners coming to the Netherlands with the demands of the labour market, given the available or yet-to-become available facilities for housing, reception and training. ${ }^{1}$

Moreover, the categorisation of migrant groups also reflected differentialism. Migrant groups were not named and framed as one category, but defined according to their foreign origins - Surinamese, Antillean, Moluccan, foreign workers - with the emphasis on the fact that they were not from the Netherlands. This was also reflected in the fragmentation across various 
departments of policy responsibilities for these groups and the fact that policy memoranda issued until the late 1970s always concerned one specific group. ${ }^{2}$ The categorisation of migrant groups as such maintained links with the countries of origin and also stressed their different migration backgrounds (colonial migration, labour migration, family migration, asylum migration). It highlighted the fact that there was no common framework for formulating a general immigrant integration policy.

The causal story underlying the policies for the separate groups was based on the idea that policies aimed at permanent integration could hamper return to the home countries. Although it became clear early in the 1970 s that the presence of foreign workers would be permanent, it was still believed that foreign workers would not become permanent minorities.

The phenomenon of foreign workers in the Netherlands has been recognised to be permanent [...] but the change among them is significant $[\ldots]$ as most of them return to their home countries after a short or a longer time. ${ }^{3}$

The presence of temporary migrants was framed as a product of post-war economic reconstruction and decolonisation. To facilitate return migration, migrants would have to be able to preserve, as best as possible, their cultural identities and internal group structures. Whereas integration was pursued in social-economic domains such as labour and income, in the socialcultural domain, migrant groups were encouraged to keep themselves apart from Dutch society. For instance, differentiated housing facilities were created (e.g. the Moluccan camps and quarters and guest worker barracks) and education was facilitated through immigrant minority language and culture classes. In a memorandum on foreign workers, the focus on retention of identity was phrased as follows:

More than on promoting integration as such, policy must be directed at providing a group with the chance to retain its identity. This means that the group is encouraged to develop its own activities. A group-focused approach is essential to this aim. [...] For foreign workers, who will generally remain in our country for only a short time, the emphasis will be mainly on retention of identity. Reintegration after return to the home country will then be less problematic. $^{4}$

Finally, policies towards migrants were framed in the context of powerful values and norms establishing that the Netherlands 'was not and should not be a country of immigration'. 5 An important argument raised in this context was that the country's demographic situation, described in terms of 'overpopulation', would make it undesirable for permanent immigration. ${ }^{6}$ 
The migration that had taken place in the 1960s and the 1970s was seen as an inadvertent consequence of economic and political developments. This norm of not being a country of immigration also provided an argument for not developing a policy for immigrant integration, as the integration of migrants could otherwise be interpreted as positive appraisal of the idea of being a country of immigration.

\subsubsection{The minorities policy}

The first official immigrant integration policy in the Netherlands was developed in the early 1980 s, initially as a draft minorities memorandum in 1981 and finally in an official minorities memorandum in 1983. Then, for the first time, the presence of specific immigrant groups was recognised as being permanent. "The new policy is based on the assumption that ethnic minorities will remain permanently in the Netherlands [...] thereby distancing itself from the idea that their presence would have been of a temporary order'. ${ }^{7}$ Migrants were also named and framed as permanent settlers. Once guest workers or colonial migrants, they were now categorised as permanent 'cultural minorities' or 'ethnic minorities', emphasising their permanent position within Dutch society. However, immigration as such was still not seen as a permanent phenomenon, and the Netherlands was still not considered a country of immigration, since the immigration of these specific target groups was framed as a historically unique event.

Assimilationism and differentialism were explicitly rejected. ${ }^{8}$ Assimilationism was at odds with the freedom of minorities to experience their own cultures. Differentialism had served too long as an excuse for the government not to create a policy on integration. The frame underlying the minorities policy had characteristics of both a multiculturalist and a universalist frame. On the one hand, policy discourse stressed 'mutual adaptation' in the context of the Netherlands as a 'multi-ethnic' or 'multicultural society'. ${ }^{9}$ On the other hand, this mutual adaptation involved not only the social-cultural emancipation of minorities and combating discrimination, but it also enhanced the social-economic participation of members of minorities. ${ }^{10}$ The merging of multiculturalist and universalist elements is also reflected in the combination of a group focus with an individualistic focus. As seen in the official policy, the aim was: 'to achieve a society in which the members of minority groups that reside in the Netherlands can, individually as well as group-wise, enjoy an equal position and full opportunities for development'. 11

The major focus of all policy documents since 1979 on the social category of 'ethnic minorities' signals the more multiculturalist aspect of the minorities policy. Migrant groups were no longer categorised according to foreign origin, but rather as permanent populations within Dutch society. Introduced thus was one common frame of reference for migrant groups as 
a whole, who up until then had been treated separately. However, the government did not provide a definition of 'ethnic minorities'. Rather, it selected a number of 'minorities' who formed the target groups of the minorities policy: Moluccans, Surinamese, Antilleans, foreign workers, gypsies, caravan dwellers and refugees. ${ }^{12}$ This list included the main groups that had emerged from labour, family and asylum migration up until that period. The selection was legitimised by the argument that the government was responsible for these minorities because 'their arrival and settlement in the Netherlands has been so entwined with the history and economic functioning of Dutch society'. ${ }^{13}$ Some migrant groups in Dutch society, such as the Chinese and Pakistanis, were left out.

In terms of the causal theory underlying the minorities policy, an important premise was that the social-cultural emancipation of minority groups would also favour the social-economic participation of their individual members. This also reflects the combined multiculturalist and universalist thinking behind the minorities policy. Multiculturalism clearly prevailed in the policy's orientation towards specific target groups. Although the slogan 'General when possible, specific when necessary' was introduced - referring to decisions on whether a minority group's position would be best ameliorated by means of general policies or targeted group-specific (categorical) measures only when necessary - the minorities policy was still mainly directed at specific groups. ${ }^{14}$ For instance, it was believed that the social-cultural emancipation of these groups could be furthered by maintaining group-specific facilities for immigrant minority language and culture classes. This, in turn, would eventually benefit individual socialeconomic participation. ${ }^{15}$ It was also believed that the democratic voice of migrants would have to be supported by developing an advisory and consultation structure between national government and immigrant organisations.

However, universalism prevailed as far as an emphasis on the accessibility of societal institutions and on proportionality in terms of socialeconomic participation. This meant that:

regulations for all inhabitants $[. .$.$] are not just formally open to inha-$ bitants from minority groups, but that they also effectively benefit minorities, which would have to be established by examining to what extent members of minority groups make proportional use of these regulations. ${ }^{16}$

Together with combating discrimination, enhancing the accessibility of institutions would constitute a means for enhancing social-economic participation.

Finally, the minorities policy was framed in terms of a multi-ethnic, multicultural society being something positive. ${ }^{17}$ Immigration was not framed as a permanent phenomenon, though the presence of ethnic minorities was 
considered permanent. However, this orientation towards multiculturalist values did not involve much by way of cultural relativism. The slogan 'Integration with retention of identity' was now abandoned, at least in official policy discourse, in favour of a more dynamic conception of immigrant cultures. This was also manifest in the emphasis on mutual adaptation. As a result of the asymmetrical relationship between minorities and the majority, the integration of minorities would inevitably require their adaptation to Dutch society to some degree. As the minorities memorandum stated, 'When values and norms of minorities from their original culture clash with those of the established norms of our pluriform society and when these are considered as fundamental for Dutch society'. ${ }^{18}$

\subsubsection{The integration policy}

The assumptions of the minorities policy stayed relatively stable throughout the 1980s. Economic depression and rising unemployment levels among minorities forced an incremental priority shift in the direction of social-economic participation. Attempts to increase the accessibility and proportionality of minorities' representation in state regulations were especially stepped up in this respect. A large-scale project to enhance the number of minority members in government service (the so-called 'EMO plan') and a project to identify and eliminate instances in which the legal position of minorities was inferior to those of natives (Beune \& Hessels 1983 ) illustrate this emphasis on accessibility and proportionality.

By the end of the 1980 s, the government started to raise doubts about whether the current approach of the minorities policy should be continued as, especially in material domains (housing, education and labour), the results proved disappointing. ${ }^{19}$ Although there does not seem to have been a radical break in many concrete policy programmes, the early 1990s saw a significant change in the discourse, categories, causal stories and values concerning immigrant integration. ${ }^{20}$ An important shift took place in a reply memorandum to a report from the Scientific Council for Government Policy (WRR), offering recognition that immigration would form a permanent phenomenon in Dutch society. ${ }^{21}$ Although the government still firmly held onto other elements of the minorities policy - including the norm that the Netherlands should not be a country of immigration and that specific minorities for whom government had a special responsibility would be the targets of the minorities policy - this reframing of the nature of immigration would have significant consequences. For instance, it raised the question of how policy could accommodate a constant influx of new migrants, beyond those minority groups that were considered the targets of policy. Moreover, rising doubts about policy effectiveness in material areas led to a shift in prioritisation from the social-cultural to the social-economic domain of integration. As an example, new plans were initiated in the 
domains of education and labour, including plans for an Employment Equity Act and educational trajectories for newcomers as a first step towards integration. ${ }^{22}$

During the debates on the 'Annual report of the minorities policy' in $1993,{ }^{23}$ a parliamentary motion was adopted that asked for a formal recalibration of government policies. ${ }^{24}$ It was argued that the notion of the minorities policy no longer covered the revised policy ideas from previous years (Koolen \& Tempelman 2003: 100). ${ }^{25}$ This set in motion a process that would lead to a more universalist type of policy framing. In response to a parliamentary motion, the government issued the 'Contours memorandum on the integration of ethnic minorities ${ }^{, 26}$ in 1994 . This would be succeeded several years later by the memorandum 'Providing opportunities, seizing opportunities' (1998). ${ }^{27}$ An important change in discourse involved the switch from the minorities policy to integration policy and emergence of the 'citizenship' concept. The focus on integration instead of emancipation (Fermin 1997: 211) located immigrant integration more from the perspective of participation in central societal institutions (education, labour, the welfare state, politics). Instead of group emancipation, individual immigrants would now have to become the unit of integration into Dutch society.

The Cabinet chooses for citizenship and thereby stresses the integration of members of minority groups into Dutch society. Hence, we will no longer speak of a minorities policy but instead of an 'integration policy' for minorities. For some, the past years have given the impression that policy would only be directed at the recognised policy target groups. Society on the whole as a target group would have remained too much out of the picture, at least in their perception. This is not beneficial for Dutch society. The term 'integration policy' better underlines how the social integration of minority groups and persons belonging to these groups is a mutual process of acceptation. ${ }^{28}$

The universalist character of the integration policy is illustrated by the social categorisation of migrants as 'citizens'. Depicting migrants as citizens testifies to the more individualistic way of problem framing. This frame's 'primary goal' was formulated as 'realising active citizenship of persons from ethnic minorities'. ${ }^{29}$ The 1998 memorandum consistently refers to citizenship as 'active citizenship'. Now reframed as citizens, minority members saw their rights, as well as duties, became more central.

On all members of ethnic minorities that stay permanently in the Netherlands [...] lies the individual obligation to participate in 
education and labour market and, also, the obligation to make efforts to learn the Dutch language and to acquire basic knowledge of Dutch society. ${ }^{30}$

Reducing policy target groups to a limited number of minority groups was not yet officially abandoned, as the targets now became individual members of those groups. It was indicated that, from time to time, the selection of target groups would be reconsidered, because of 'the differentiation within and between the target groups' and the 'significant progress' in the position of specific groups, such as foreign workers of Southern European origin. ${ }^{31}$ However, the 1994 contours memorandum announced the development of elaborate civic integration programmes for newcomers, as immigration was now considered a permanent phenomenon. This meant that the target population of this aspect of the integration policy was no longer restricted to the selected minority groups.

The priority shift from the social-cultural to the social-economic dimension of integration reveals a more significant change in the underlying causal policy theory. First, integration problems were no longer primarily perceived in terms of accessibility of societal institutions, but now also in terms of the individual rights and duties of migrants as citizens. As a result, the slogan 'Providing opportunities, seizing opportunities' was introduced. Second, the theory that social-cultural emancipation would eventually also benefit social-economic participation was reversed. Socialeconomic participation was now considered an important condition for social-cultural emancipation. For example, immigrant minority language classes were now legitimised by the argument that mastering one's own mother tongue would facilitate the apprehension of a second language. Third, integration policy was increasingly framed as an intensification of general policy in specific domains, rather than a specific policy for specific groups. Specific policies would only be conducted temporarily in specific domains, such as the employment equity law to promote proportional labour participation of migrants. For the most part, however, integration would have to be an intensification of general social affairs, labour, education, housing and health care policies. In particular, integration policy was considered to be related to the policy of social renewal, which involved a clearly decentralised approach to a variety of urban social problems. As the policy of social renewal shifted to the background in the late 1990s, integration policy became more closely related to urban policy.

Finally, policy documents show evidence that value of the Netherlands as a multi-ethnic or multicultural society receded to the background in the 1990s. Although the government still recognised the de facto multicultural status of Dutch society, it no longer considered the active promotion of such a society a facet of government policy. This is put in the perspective of 'the changing role of the government' and recognition that 'more parties 
than just government are responsible for the dilemmas of the multicultural society, ${ }^{32}$ Rather, government policy was to be restricted to the sphere of social-economic participation, not least because of rising concerns about the viability of the welfare state in relation to immigration. A deteriorated economic climate, the permanent immigration of new immigrants and too little attention for the problems of socio-economically deprived native citizens has made mutual adaptation and the support for an integration policy less obvious. $^{33}$

\subsubsection{The integration policy 'new style'}

The universalism of the integration policy allowed for a more assimilationist type of policy framing after the turn of the millennium. Once again, a significant change took place in the discourse, categories, theories and values used for depicting the issue of immigrant integration. In the memorandum 'Integration in the perspective of immigration', the government indicated that integration policy would have to be recalibrated in the context of recent events (such as the 9/11 terrorist attacks and a broad national debate on immigrant integration triggered in the media by a newspaper article by Paul Scheffer), which had fuelled concerns about public support for the integration policy. ${ }^{34}$ As a first step to policy reframing, this memorandum established a more systematic connection between immigration and integration policy. Immigration would have to be adjusted as far as the extent to which immigrants would be effectively integrated in Dutch society, with civic integration courses acting as a crucial link between immigration and integration.

A memorandum in response to a report from the Social and Cultural Planning Office (SCP) in 2003 marked a significant frameshift. ${ }^{35}$ In this memorandum, the minister of immigration and integration described the contours of a so-called integration policy 'new style', which involved a turn from universalism towards assimilationism. The philosophy of this policy would be elaborated further in a reply memorandum to an advisory report from a temporary parliamentary research committee on the integration policy. ${ }^{36}$ Whereas integration policy had focused primarily on socialeconomic participation, the focus now shifted towards social and cultural distance between migrants and Dutch society. ${ }^{37}$ This complicated issues because 'when groups are put up against each other - as societal institutions are not sufficiently effective for ethnic groups and as large parts of the minority population do not actively participate in the economy - the continuity of society is at stake'. ${ }^{38}$ In order to support 'the continuity of society', focus must be on the bridging of differences rather than on 'the cultivation of one's own cultural identities'. 
The integration policy has always put great emphasis on the acceptance of differences between minorities and the native population. There is nothing wrong with that, but it has often been interpreted as if the presence of allochthonous minority groups in itself would have been valuable, an enrichment tout court. One disregards that not everything that is different is also valuable. With the cultivation of one's own cultural identities it is not possible to bridge differences. The unity of our society must be found in what the members have in common. That is, [...] that they are citizens of one society. Common citizenship for allochthonous and autochthonous residents is the goal of the integration policy. [...] Common citizenship means that people speak Dutch, and that one abides to basic Dutch norms. $^{39}$

In terms of social classification, categorisation of a limited number of minorities was now abandoned. All newcomers as well as long-term resident migrants, so-called 'oldcomers', were to be target groups of the integration policy, regardless of ethnic or cultural origin. All newcomers were obliged to follow 'civic integration programmes' after their arrival in the Netherlands. The tone regarding immigrants, however, had become increasingly negative, as demonstrated by the statement that 'not everything that is different is also valuable' (see quotation above). In other words, the social construction of migrants a as target group became more and more negative. Citizenship remained the primary means for categorising minorities. However, the focus shifted from 'active citizenship', with a strong universalist implication, to 'common' or 'shared citizenship', with a more assimilationist meaning. Common citizenship involves a form of citizenship based on common values and norms; it involves 'speaking Dutch and complying with basic Dutch norms, [such as] doing your best to provide for your own life support and observing laws and regulations'. It brings with it a willingness for 'taking care of the social environment, respecting physical integrity of others, also within marriage, accepting the right of anyone to express one's opinion, accepting the sexual preferences of others and equality of man and woman'. ${ }^{40}$ Also, it maintains some of its universalist traits, i.e. that citizens are individually responsible for their participation in society.

Rather than social-cultural emancipation being a condition for socialeconomic participation (as assumed by the minorities policy) or socialeconomic participation being a condition for social-cultural emancipation (as assumed by the integration policy), the new causal story stated that social-cultural differences could form an obstacle to social-economic participation. Diminishing social and cultural distances between migrants and natives would support the participation of migrants in society and would eliminate problems such as criminality and rising social tensions in 
neighbourhoods with high concentrations of immigrants. Just as with the integration policy, the individual migrant remained the main unit of analysis. 'A lot would depend on the own efforts' made by the immigrants. ${ }^{41}$ What was to be avoided was immigrants becoming a 'welfare category'.

Integration policy runs the risk of treating minorities as a population category that is more or less in need of help, as a welfare category. That can happen when the emphasis is too much on providing facilities and offering regulations and arrangements. The Cabinet is determined to make important changes on this point. A new division of responsibilities has been made between national government, local government, civil society and individual native, allochthonous citizens. $^{42}$

No mention is made of the notion of the Netherlands as a multicultural society. Instead of interpreting growing cultural diversity as a sign of a multicultural society, cultural differences are now framed as problematic cultural distances. ${ }^{43}$ It is argued that 'a too large proportion of minority groups live at too great a distance from Dutch society'. In this context, the goal is to 'diminish the distance between minorities and the native population in social, cultural as well as economic respects'. ${ }^{44}$ Immigrant integration was now not merely about ameliorating the position of migrants within society, but also about the consequences of migration and integration for 'the continuity of society'. In other words, the normative perspective underpinning integration policy 'new style' had more to do with concerns about national social cohesion and national identity than earlier policies.

\subsection{Frames in immigrant integration research}

Research on immigrant integration in the Netherlands has followed a development path that is, on many counts, strikingly parallel to that of policies. Since the 1970s, a thriving field of research - a 'research industry' has evolved, situated not just in various centres or institutes, but also in numerous government-oriented advisory bodies. In this regard, several models or problem frames can be discerned, rather than there being just one dominant model. However, an important difference between policy discourse and research is that the latter seems characterised by a growing fragmentation between frames of immigrant integration, rather than by a succession of models. 


\subsubsection{The birth of immigrant integration research}

Until about the 1960s, the presence of immigrants attracted little attention from researchers (Penninx 1988b: 255; Rath 1991: 274). Like policymakers, researchers named and framed immigrants as temporary migrants. Lucassen and Köbben (1992: 84), two pioneers in this field, would later observe how this showed that 'policy makers as well as researchers are part of the same society and are both subject to the same general if not ideologically influenced ideas'. For instance, in a study of foreign workers, Wentholt (1967) depicted migrants as 'international commuters'. Or, in a study commissioned by CRM on migrant groups in the Netherlands, the term 'immigrant' was avoided and deleted from the study's working title to prevent perception that these migrants would be permanent (Van Amersfoort 1984: 148). Instead, the study employed 'allochthonous'(Verwey-Jonker 1971), a concept to later acquire a somewhat different meaning.

Initially, the causal story regarding the position of migrants in the Netherlands also reflected the idea of temporary residence. For example, one of the largest government projects, involving many researchers, was aimed at examining the possibilities for linking return migration to the development of the countries of origin (the Reintegration of Emigrant Manpower and Promotion of Local Opportunities and Development Project, REMPLOD). Many researchers who played a major role in the development of the immigrant integration research field were initially involved in this project (including Penninx and Bovenkerk). Another example concerns a study by the Dutch Statistics Foundation (Statistiek 1971) that failed to calculate the economic effects of permanent immigrant settlement, thus leading to positive recommendations about the economic effects of labour migration (Tinnemans 1994: 104). Also, studies of the position of migrants during their stay in the Netherlands often stressed the psychological and social difficulties they experienced when arriving in the Netherlands from a very different social-cultural context, calling for forms of social assistance to help migrants 'acclimatise' to the Netherlands (Van der Velden 1962 in Rath 1991: 152).

Finally, an important characteristic of the values held by researchers involved in the initial development of this field was the strong sense of engagement with the position of minorities. For instance, Köbben and Penninx, who would later become key figures in immigration policy, were engaged in organisations that aimed to alleviate and ameliorate the position of minorities. Köbben and Mantouw led a committee to discuss the position of Moluccan migrants in the late 1970s, while Penninx held action groups for foreign workers. ${ }^{45}$ Another prominent researcher, Bovenkerk carried out extensive work to uncover patterns of discrimination in Dutch society (Bovenkerk 1978). 


\subsubsection{The minorities paradigm}

In the 1970s especially, immigrant integration began attracting attention from researchers in a variety of disciplines, such as anthropology, sociology and social geography. A landmark study for the development of this domain was published by Van Amersfoort in 1974. Entitled 'Immigration and minority formation', it defined immigrants for the first time as ethnic or cultural 'minorities', reflecting American sociological literature on minorities. Van Amersfoort referred to ethnic minorities as social groups or 'collectivities' who had strong internal bonds, problems with political participation and weak social positions. In this respect, he called for government intervention to prevent what he dubbed as 'minority formation'. This meant that specific policies would be needed for specific collectivities so as to circumvent their forming a minority. Defining minorities and minority formation in this way provided the basis for research on minorities in the 1970s. The framework was as follows:

1. A minority forms a continuous collective within society. The continuity of a minority has two aspects: (a) the minority encompasses multiple generations; (b) belonging to a minority has priority over other social bondings.

2. The numerical position of a minority hampers effective participation in political decision-making.

3. The minority takes in an objectively low social position. (Van Amersfoort 1974: 37)

In the mid-1970s, more studies and articles emerged to raise doubts about the differentialist frame that had prevailed thus far, calling instead for a different frame and approach to immigrants. In addition to Van Amersfoort's (1974) study on minority formation, an article by Entzinger (1975) on norms versus facts of being an immigration country and a study by Bovenkerk (1974a), raised doubts about the feasibility of return migration. Köbben (1986: 157) observed that the 'minorities' concept had become the common denominator for the various minority groups from the second half of the 1970s onwards. Thus, Van Amersfoort's study provided the fundamentals for a 'minorities paradigm' (Penninx 1988a: 23; Rath 1991: 173) that was elaborated on by researchers, particularly those with an anthropological or sociological background, such as Penninx, Bovenkerk, Köbben and Entzinger. Rival paradigms, such as nationalist and Marxist perspectives on immigrant integration, had become marginal by the end of the 1970s (Bovenkerk 1984; Rath 1991). The establishment of the government-associated Advisory Committee on Minorities Research (ACOM), which united most of the researchers in the then still relatively small field, 
would play an important role in research coordination and distribution of research funding (Penninx 1988a; Rath 1991; Van Putten 1990).

The naming and framing of ethnic minorities was closely related to an underlying causal theory of minority formation. Elaborating on Van Amersfoort's pioneering work, Penninx (1988a: 55) defines minority formation as a process involving 'position attribution' by structural factors in society and 'position acquisition' by migrants. Position attribution involves issues such as discrimination and accessibility of institutions, whereas position acquisition involves educational and labour market qualifications and cultural orientations of migrants. The central explanation for minority formation in this model is that the social and cultural 'otherness' of minority groups can negatively impact position acquisition and attribution for minorities. For instance, a study by Veenman (2001) observes that the weak social position of Moluccans tends to be reproduced by weak educational achievements. Or, the cultural 'otherness' of foreign workers would trigger systemic discrimination that would lead to negative position attribution. A central characteristic of the minorities paradigm is that position acquisition and attribution are examined primarily on the level of migrant groups; the social-economic and social-cultural characteristics of minorities are believed to determine the position of minority group members.

Finally, mirroring the rise of the immigrant integration field, research in this period generally carried a value orientation that closely engaged with the position of minorities. For instance, in the late 1970s, researchers played a leading role in calling for a minorities policy that would recognise the permanent status of minorities and ameliorate their social position in Dutch society (Entzinger 1975; Scientific Council for Government Policy 1979). The very notion of 'minorities' emphasised migrant groups' permanent position in Dutch society, rather than being commuters or guest workers. Also, researchers generally accepted, with little debate, that the Netherlands had become a de facto multicultural or multi-ethnic society. Their goal was to promote the cultural emancipation of minorities within this multicultural society by describing and analysing the process of minority formation and drawing attention to the relation between group characteristics and processes of position acquisition and attribution (Rath 1991: 36).

\subsubsection{The integration paradigm}

Although the minorities paradigm did not disappear, it was challenged by rival paradigms in the late 1980 s and early 1990 s. Its dominant position was undermined by 1992's discontinuation of ACOM, which had a central role in the field structure. The most significant rival paradigm has been described as the 'citizenship paradigm' or 'integration paradigm' (Favell 2005: 46). Once again, we see how this paradigm named and framed the 
integration of migrants as 'citizens' in key societal institutions, such as education and the labour market. To some extent, this line of research had already been present for a considerable time, though had remained quantitatively less important than the more cultural-anthropological line of research (Choenni 1987).

The integration paradigm first gained prominence in a WRR report. Entitled 'Allochtonenbeleid' (1989), it suggested replacing the concept of 'minorities' with 'allochthonous', so as to avoid putting too much emphasis on the group dimension of integration. The report also called for more proactive social-economic participation by immigrants in order to prevent them from becoming welfare categories. Migrants would have to be able to stand on their own feet instead of being dependent upon government facilities (WRR 1989: 17). Although the notion of 'citizenship' was not yet employed in this report, it would become the central concept in later studies from a similar perspective (Engbersen \& Gabriëls 1995b; Van der Zwan \& Entzinger 1994). In the early 1990s, the Social and Cultural Planning Office (SCP) also attributed more systematic attention to the socialeconomic position of migrants. In this period, SCP measured the socialeconomic position of migrants using generic data and also determined the proportionality of migrant participation in direct comparison with data about social-economic participation in society at large. This differed from research undertaken by the Institute for Social and Economic Research (ISEO), which gathered specific data about migrant groups by using certain quantitative methods that SCP would go on to adopt at a later date. ${ }^{46}$

The focus on citizenship and social-economic participation also revealed the changing underlying causal story. Not being able to be self-sufficient was now considered the main explanation for integration problems, and the welfare sensibility of the minorities policy was blamed for creating this relationship of dependency. This can be interpreted as a shift away from the group to the individual level, as well as a shift from structural factors in society affecting the position of migrants to the individual qualities of migrants (i.e. from position attribution to position acquisition). In the literature, this new paradigm has been described as a form of 'new realism', because of its realist tone on issues of integration (Prins 1997). The minorities paradigm addresses issues of immigrant integration head-on, with immigrants being called to live up to their civic responsibilities.

The call for new realism in the Netherlands can be seen as a response to, on the one hand, a Dutch governmental policy of 'care', motivated and initiated since the 1970 s by Christian and social-democratic governments, and, on the other hand, the demand for politically correct representations of social reality from the radical left. The advocates of new realism think these standpoints are often too soft on members of minority groups, and that they put an unjustified 
taboo on critically questioning their different habits, cultures and beliefs. (ibid.: 118)

New realism also points to an important normative element of the citizenship paradigm. It calls for full and equal citizenship of migrants, without treating migrants too much as 'dependents' and without taboos that would complicate the study of immigrant integration. Prins (ibid.: 117-142) lists several characteristics of new realism, including the assertion that immigrant integration should be treated with courage instead of care. This new realism claims to represent the voice of the common people that, thus far, had been left unheard. It claims that by engaging in serious debates about integration and cultures, immigrants are taken more seriously. As such, this perspective sought to eradicate alleged taboos surrounding the debate on social-economic participation of minorities and on the role of their socialcultural backgrounds.

\subsubsection{Transnational frames versus national frames}

The immigrant integration research field was becoming increasingly fragmented around the turn of the millennium. Alternative frames emerged to breach the 'academic provincialism' (Rath 1991; Van Amersfoort 1984) that had characterised research to this point, by bringing the state 'back in' and stretching the perspective beyond the borders of the nation-state. More and more, research in the Netherlands, as well as in many other countries, acquired a pronounced European dimension (Geddes 2005). A criticism of the integration (and minorities) paradigm concerned its confinement to the context of the nation-state without questioning the nation-state - that research had focused primarily on the integration of immigrants as citizens within nation-states (Favell 2005). In this respect, national research paradigms were often associated with nation-building legacies (Lavenex 2005).

By the end of the 1990s, the national dimension was increasingly questioned in a growing body of literature that adopted transnationalist or postnationalist frames (Council for Public Government (ROB) 2001; Council for Social Development (RMO) 2005; Entzinger 2002; Entzinger \& Van der Meer 2004; Scientific Council for Government Policy 2001b; Snel \& Engbersen 2002; Van Amersfoort 2001). For instance, WRR adopted a transnationalist frame by naming Dutch society an 'immigration society'. It drew attention to the formation of transnational communities and causally linked migration and diversity to the transformation of society. Furthermore, the council adopted a normative perspective that migration and diversity were inescapable facets of the ongoing process of modernisation that should benefit the receiving countries (WRR 2001b). Entzinger (2002) also drew attention to the formation of these transnational communities and to the emerging reality of dual identities among migrants. Snel 
and Engbersen (2002) describe transnational citizenship as a new form of citizenship that bonds migrants to both their countries of origin and their destination. Finally, Entzinger and Van der Meer (2004) draw attention to how immigrant integration involves an adaptation of the host society's institutions, for example, the adaptation of welfare state arrangements. Transnationalist problem framing can be clearly observed in this literature, which stresses the formation of transnational ties (migration links, dual nationalities, dual identities), defining migrants as transnational citizens, explaining immigrant integration in terms of both participation and the transformation of national institutions in response to migration and, finally, linking immigrant integration to normative processes of internationalisation and globalisation.

The turn of the millennium also saw a rise in studies with a more assimilationist problem framing. This included SCP studies that gradually shifted from universalism to assimilationism. At this time, SCP simultaneously pointed to the progress that would have been made in the social-economic domain and drew increasing attention to the lack of progress in what it described as 'social-cultural integration' (SCP 2003). Moreover, it rejected the claim that the Netherlands would have become a multicultural society and started to look at more social-cultural explanations for immigrant integration, such as criminality, gender equality, residential segregation, social contacts and language proficiency (SCP 1998, 2002). Along with SCP, other researchers, such as Koopmans (2003; Koopmans et al. 2005), were at odds with the emerging transnationalist perspectives. According to Koopmans, immigrant integration remained primarily a national process, as there was little evidence of transnational claims being made by migrants themselves. He also believed that the absence of an effort to achieve social-cultural integration - for instance, because of resilient pillarist tendencies to accommodate cultural differences - would serve as one of the explanations for stagnating integration. In contrast to the transnationalist literature, these studies name and frame immigrant integration in terms of social-cultural integration and social cultural issues. They define migrants as transnational citizens; they focus on the social-cultural adaptation of migrants instead of the adaptation of national institutions and, finally, they place integration within a normative perspective of preserving national social cohesion and national identity in an era of globalisation and internationalisation.

\subsection{Conclusion}

This chapter has shown how immigrant integration developed into an intractable controversy in research and policy over the past decades. Both immigrant integration research and policy were increasingly marked by 
uncertainty in terms of problem framing. In immigrant integration policy, the problem framing changed about once every decade: from a differentialist frame until the $1970 \mathrm{~s}$, to a multiculturalist frame with universalist traits in the 1980s, a universalist frame in the 1990s and, finally, a more assimilationist frame after the turn of the millennium. This illustrates the inconsistency of policy in this domain. Furthermore, the policy frames often differed and even conflicted in various ways. For instance, the differentialist policies of the 1970 s were clearly at odds with the more integration-oriented policies of later periods. Or, the policy directed at emancipation of ethnic minorities was at odds with the more universalist approach of the 1990 s, as well as with the assimilationist approach from after the turn of the millennium.

No doubt there has been a growing fragmentation in problem framing in immigrant integration research. Although the scarce research on this topic up until the 1960s regularly followed a differentialist frame, in the 1970s a dominant minorities paradigm was established that contained a multiculturalist problem framing with some traits of universalism. In the late 1980s and early 1990s, a rival perspective emerged (the integration or citizenship paradigm) that contained a more universalist problem framing. Finally, in the late 1990s and after the turn of the millennium, transnationalist and post-nationalist frames exacerbated the fragmentation. At the beginning of the twenty-first century, no dominant research frame existed, thus strengthening the observation that, in the research field as well, immigrant integration had become an intractable controversy.

Furthermore, the frameshifts in research and policy seem to have overlapped. In both research and policy, a multiculturalist problem framing became dominant from the late 1970 s to the early 1980 s. This period would have been notably affected by the minorities memorandum (1983) and ACOM and WRR reports. Later, more universalist frames emerged in both research and policy during the same period, between the end of the 1980s and the early 1990s. In policy, a new memorandum marked this shift in 1994; in research, this period involved various reports, including a second report by WRR in 1989. Finally, the rise of transnationalist and assimilationist frames in research and the rise of assimilationism in policy also seem to have been more or less parallel developments, primarily taking place around the turn of the millennium and immediately after. In this period, two government memorandums on the integration policy 'new style', a report from a parliamentary investigative committee, several reports by SCP and a third report by WRR appear to have played important roles.

The following chapters will zoom in on each of the periods during which frameshifts took place and new frames emerged in immigrant integration research and policy. They will analyse empirically how and why these frameshifts occurred in research and policy, particularly looking to explain the role that the research-policy nexus served in these shifts. 


\section{Technocracy and the construction of the Dutch multicultural model (1978-1983)}

The preceding chapter illustrated how a multiculturalist problem frame emerged in both policy and research during the end of the 1970s and the early 1980s. The approach was to become internationally renowned termed the 'Dutch multicultural model'. This chapter will venture into the strategic and symbiotic co-evolution of this frame in policy and research. More precisely, it examines the minorities policy and the minorities research paradigm.

As will be seen, the configuration of research-policy relations in this period played a major role in keeping the Dutch debate on immigrant integration 'behind closed doors' (see e.g. Guiraudon 1997). Indeed, as the literature reveals, during this period the configuration of research-policy relations was highly technocratic. What has yet to be analysed is how and why this technocratic research-policy nexus evolved at this time and how it contributed to the construction of the Dutch multicultural model. This technocratic nexus is often associated with a tendency for depoliticisation. The situation, however, is more nuanced, as shown by the strong convergence between researchers and policymakers at this time in terms of their belief in rational societal steering. Furthermore, the nexus created a very specific logic of problem framing that provided the structural basis for a multicultural model.

\subsection{The rise of a multiculturalist model}

Primary questions to be addressed at this point are: how did the shift from differentialism to multiculturalism take place? Which research and policy actors were involved in it? And what were the positions and frames of those actors? This analysis will reveal how even at the point in time when the now famous Dutch multicultural model emerged, a multiplicity of frames existed. Being advocated by varying actors at the time, the many frames complicated the debate on immigrant integration. Moreover, in the context of research-policy dialogues, the selection and interpretation of relevant research findings differed greatly among the actors. In fact, the rise of the Dutch multicultural model was far less self-evident than has been suggested. As will be discussed, a very particular configuration of the 
research-policy nexus was to play a central role in taming the ongoing frame controversies and construction of the first dominant policy and research paradigms in this field.

\subsubsection{From differentialism to multiculturalism}

Until well into the 1970 s, a differentialist framing of migration accounted for both the absence of a minorities policy and of more significant research interest in this issue. There was a belief in both the policy and research domains that an immigrant integration policy was superfluous, as (most) immigrants would return to their home countries. Researchers showed little interest in immigrant integration (Penninx 1988b: 18-20). Early 1970s memoranda originating from those policy divisions responsible for specific groups of 'temporary' migrants - such as the Department of Social Affairs (guest labourers), Department of Culture, Recreation and Social Work (Surinamese, Moluccans) and Department of Foreign Affairs (asylum applicants) - state clearly that the Netherlands was not and should not be a country of immigration.

However, a series of developments took place in the 1970s that would prompt a number of actors to question this differentialist frame. Firstly, even after the active recruitment of foreign labour had been halted following the oil crisis of 1973, immigration continued. The oil crisis marked the beginning of an economic reconstruction that would significantly affect labour intensive industries (textiles, mining) in which many immigrants were employed (De Beer 1998: 242). However, many foreign labourers did not return to their home countries and, instead, brought their families over to the Netherlands. Notably between 1977 and 1981, family migration led to a steep growth of specific migrant groups, including Turks and Moroccans (Koolen \& Tempelman 2003: 26). On top of this, the independence of Surinam brought two more waves of immigration to the Netherlands: one in 1975 at the time of the country's formal independence and one in 1980 when Surinamese were given a final opportunity to qualify for Dutch nationality. The end of the 1970 s consequently marked a period with unprecedented immigration levels.

Secondly, the growing presence of migrants in Dutch society contributed to a series of events that would bring attention to the issue of immigrant integration. For the first time since those that took place in the region of Twente in the 1960s (Groenendijk 1990b), ethnic riots occurred in 1972 and 1976 in the cities of Rotterdam and Schiedam (Donselaar \& Wolff 1996). The simultaneous emergence of several anti-immigrant and extreme-right parties in the early 1970s caused considerable public arousal. Among those was the Dutch National People's Union, which put candidates up for the municipal elections in The Hague in 1982. Another extreme-right organisation called the Centre Party caused much consternation 
by gaining wide support in Almere, not only in the neighbourhoods primarily populated by labourers where extreme-right parties traditionally find approval, but also in the city's middle-class areas.

Furthermore, a series of terrorist acts was committed by Moluccan migrants in the 1970s. At that time, the Moluccan community had already been living - 'temporarily' - in the Netherlands for over a quarter century, without any clear prospect for either return or integration. Since the 1950s, their position had deteriorated from one of stagnation, to one of marginalisation and radicalisation (Smeets \& Veenman 2000). The terrorist acts involved two train hijackings in 1955 and 1957 and, in the same year, the hostage-taking of an Indonesian consulate and an attempt to kidnap the Dutch queen. In 1958, Drente's provincial offices were also taken hostage, though the most notorious action may have been that year's hostage-taking of a class at a primary school, which received widespread attention and resulted in a great deal of public and political debate (Bootsma 2000). In response, the government decided to review its policy towards Moluccans (Koolen \& Tempelman 2003: 12). A memorandum issued in 1978 illustrates the government's efforts to integrate Moluccans. ${ }^{1}$ The impact of the events included raising a general awareness of how various immigrant minorities were positioned in Dutch society (Lucassen \& Köbben 1992: 84-85). In addition to a number of negative effects, such as declining levels of tolerance in society and waning support for the political aims of the Moluccans, the terrorist acts had a series of positive effects, such as increasing awareness about the deplorable position of minorities, the multiethnic character of Dutch society and the demonstrative effect that a new approach towards the Moluccan community could have on other minority groups (Köbben 1979).

Subsequently, various actors cited a growing 'tension between norm and fact' regarding whether or not the Netherlands was a country of immigration (Entzinger 1975). In addition, researchers began to pay systematically more attention to minorities in Dutch society. In 1978, ACOM was established to advise on research programming and to coordinate research on minorities. In the late 1970 s and into the 1980 s, the committee provided an important stimulus to the development of research in this domain (Entzinger 1981; Penninx 1988b; Van Putten 1990). ACOM was affiliated to CRM and, despite reluctance on the part of other government departments, CRM was leading the way in advocating a changed perspective on immigrant integration. Welfare and migrant organisations also played an important role as representatives of the involved minority groups in the late 1970s. These organisations also called for more systematic attention for minorities, although they less emphasised the commonalities of various migrant groups' social positions. Furthermore, the events and developments of the 1970s placed immigrant integration firmly on the political agenda. In 1978, a parliamentary motion was issued calling for a general policy on 
the integration of ethnic minorities. In response, the government established a directorate, led by Henk Molleman, for the coordination of a minorities policy at the Department of Home Affairs. According to Köbben (1979), the Moluccan terrorist acts had a direct influence on the establishment of this directorate.

A report by WRR, entitled 'Ethnic minorities', was a direct stimulus to the development of a minorities policy by Molleman's directorate (1979). The contours of the minorities policy were specified for the first time in the 1980 reply memorandum to this report, which was elaborated into a draft minorities memorandum a year later. Ultimately, after consultation with welfare and migrant organisations, this became the final minorities memorandum in 1983. These memoranda developed a multiculturalist perspective on immigrant integration, in contrast to the differentialist approach of the 1970s. At the same time, in the field of research, ACOM and WRR were advocating a more multiculturalist approach.

This brief reconstruction indicates the involvement of several research and policy actors in this period. Firstly, in the field of research, ACOM and WRR seem to have played an important role. Both published reports (ACOM 1979; WRR 1979) that are often seen as turning points in this domain (Entzinger 1984: 95; Penninx 1988b: 22). Secondly, in the field of policymaking, there were various policy departments engaged with this issue, including CRM and the Departments of Social Affairs, Foreign Affairs and Home Affairs (Entzinger 1984: 107). Welfare organisations can also be distinguished as a group of actors who were involved in policymaking. This calls first for an analysis of these actors' positions within their fields and their problem frames of immigrant integration and, subsequently, an evaluation of their boundary work.

\subsubsection{The Advisory Committee on Minorities Research (ACOM)}

$\mathrm{ACOM}^{2}$ was formally established in 1978 to advise the Dutch government on research coordination and funding. Established by CRM, it was a direct product of government efforts in the 1970s and 1980s to strengthen its role in research programming, which was also manifest in other domains (Van Hoesel 1984). ACOM consisted, primarily, of 'independent experts'. This came in contrast to other research committees that often had a 'tripartite' character, composed of researchers and the representatives of involved groups and government departments (Entzinger 1981). ACOM had a fulltime secretary, whose position was financed by CRM. In addition, a civil servant from CRM acted as an observer at ACOM meetings and functioned as a trait-d'-union to the department. By nature, ACOM was a 'technicalscientific committee' (Penninx 1988b: 22). Its role was to advise CRM on research programming, on the scientific quality of research proposals, to coordinate research relations between research projects, to monitor the 
progress of these projects and to provide policy advice based on research (Entzinger 1981: 108).

ACOM enjoyed a rather exclusive position within the field, as it brought together almost all those researchers involved in the immigration domain. It consisted of nine experts from different disciplines. Köbben was one of the experts to enhance the authority of ACOM. Taking a central role in the committee's establishment and serving as its chair (Van Putten 1990), Köbben enjoyed respect among policymakers and researchers alike. This was borne from his prior involvement in a special commission set up in the 1970s to discuss the Moluccans in the Netherlands - the KöbbenMantouw Commission - as well as his pioneering work in immigrant integration research.

In its first report, 'Minorities research advice' (1979), ACOM provided both government research programming and more general policy advice (Entzinger 1984; Penninx 1988b). The report was a departure from the prevailing differentialist problem framing of government policy. It named the Netherlands a 'de facto country of immigration' and a 'multi-ethnic society', and it adopted the social classification of immigrants as 'cultural minorities' or 'ethnic minorities', a classification developed by Van Amersfoort (1974) several years before. This document presented a causal theory: if the Dutch government were to take no specific measures to promote the emancipation and social-economic participation of cultural minorities, then minority formation - or the development of an ethnic underclass - would prove inevitable. From a normative perspective, the leading principle should be that each individual and every group have equal opportunities for participation within the context of the law and the correct application of existing (and, if necessary, new) rules (Van Amersfoort 1974: 7).

ACOM developed this frame in the context of what it saw as a growing tension between norm and fact concerning the status of immigrant settlement. In contrast to the norm that immigration should be temporary, it saw migrants settling permanently as fact. Whereas from a differentialist perspective, the growing presence of migrants was seen as a temporary phenomenon (as in the case of guest labourers), from a multiculturalist perspective, the migrants were framed as permanent ethnic or cultural minorities within society. Entzinger, who was then secretary of ACOM and one of the authors of the 1978 report, had drawn attention to this tension in an influential article several years before during his role as an administrator for CRM. The article claimed that:

the Netherlands, partly due to government intervention, has become a country of immigration, while that very same government believes it should not be, [presumably out of] fear of overpopulation and fear of minority problems on a scale comparable to some European states and the US. (Entzinger 1975: 327) 


\subsubsection{The Scientific Council for Government Policy (WRR)}

Another actor that played an important role during this period was WRR. The council was established in 1972 with the ambitious task of designing 'a future vision for society for long-term policy development [...] identify problem areas [...] that necessitate prioritisation [and] coordinate government scientific institutes [...] so as to avoid overlap and lacunae' (Van Veen in Hirsch-Ballin 1979: 13). ${ }^{3}$ WRR was to provide scientific advice on a variety of policy topics from a multidisciplinary perspective and with a long-term time frame. Initially, WRR was created on a temporary basis; however, in 1976 it was formally established through legislation.

WRR's position - straddling scientific research and policy - was a reflection of more general cultural and structural developments within Dutch social sciences and politics in the 1960s and early 1970s. On one hand, the Dutch social sciences were fairly attuned to the needs of policymaking and to the country's political culture (see e.g. Blume et al. 1991; Gastelaars 1985). There was a concerted effort among social scientists to 'become more closely involved in government policy', in a way that would not only provide scientific information, but also address fundamental policy questions (Adriaansens 1997: 23-26). For instance, the Royal Dutch Academy of Sciences was majorly involved in the institutionalisation of the social sciences in policy formulation.

Yet, on the demand side of policymaking, there were structural and cultural factors favouring scientific involvement in policymaking. In structural terms, the fragile coalitional character of Dutch politics and its structural imperative of consensus-seeking contributed to scientific research being an impartial and objective source of expertise in policymaking (Den Hoed 1995). This structure of consensus-seeking did not, for example, allow for the establishment of a WRR that would be too closely associated to a particular government department, as this would contribute to asymmetry among various political leaders. What it did allow for, however, was establishment of an independent and 'scientific' WRR, which, relying on an external source of expertise, was more distanced from government and could support consensus-seeking. Using social science for consensus-seeking has often gone hand in hand with Dutch pillarism; scientific expertise was regularly applied to depoliticise issues that could threaten inter-pillar stability (Lijphart 1968). In cultural terms, there was a strong belief in the policy relevance of social sciences. This was illustrated by the so-called 'committee on the preparation of research for the future structure of society' (also known as the De Wolff Committee), which, in 1970, advised establishing a planning council - the future WRR - for 'the scientific preparation of policies aimed at the establishment of systematic and consistent policies'. This would 'contribute to the rationalisation of the debate about policy problems' (De Wolff in Hirsch-Ballin 1979: 10-12). 
By the time WRR decided to take up the issue of 'ethnic minorities' in a report to the government, its task had been somewhat modified in response to Parliament's criticism of its allegedly impure, technocratic role in Dutch politics (Hirsch-Ballin 1979: 140). As a result, WRR would no longer provide 'advice' but only 'scientifically sound information on developments that affect society on the long term'. ${ }^{4}$ It was considered 'the task of the Cabinet to prepare a consistent policy [...] not a task of the WRR'. ${ }^{5}$ WRR would, however, provide information 'to the aid of government policy', opening up possibilities for relations with Parliament (Hirsch-Ballin 1979: 168; Scientific Council for Government Policy 1977: 14). Finally, a regulation was passed that required the government to respond to WRR studies within a maximum of three months, so as to enhance the transparency of how policy utilised these reports and increase the possibilities for parliamentary control on government application of WRR reports (Hirsch-Ballin 1979: 177). ${ }^{6}$ As research has shown, WRR adopted a rather moderate role in the 1970s. On the whole, it accepted and followed the general contours of policy and provided scientific information within them (Hirsch-Ballin 1979).

As a boundary organisation, WRR's delicate relation to broader developments in research and policy is also reflected in its internal organisation (Adriaansens 1997: 39). For instance, council members are selected based on disciplinary background as well as political affinity. In this way, the council reflects the spectrum of political parties and of various scientific disciplines. While appointment of the council chairman is a political choice, it is based on scientific authority. ${ }^{7}$ The decision to replace the entire council every five years encompasses an element of proximity as well as distance to politics and science. It creates a certain discord within the four-year cycle of Dutch politics, allowing members to maintain closeness to the scientific field, but also adapt to the changing social and political environment. Furthermore, whereas WRR's research agenda is formally decided upon by the council itself, in practice, it is discussed with the Prime Minister. Research topics are selected according to scientific and political criteria, such as whether an issue lends itself to political intervention (WRR 1988: 5-6). The government can also issue advisory requests to WRR, though WRR is not obliged to indulge them. Moreover, there are internal structures for resolving disagreements and conflicts that could threaten unanimity of support for a report in the council. Council members can choose to take a minority position, which can also be a way of putting pressure on the council to adopt specific changes. There is a hierarchical relationship between the council and WRR staff and, finally, the chairmen and secretaries of specific projects often play an important role in ensuring that agreements be reached.

In 1978, just after establishment of the second council of this then still relatively young organisation, WRR decided to take up the issue of 
immigrant integration. Like ACOM, WRR reframed immigrant integration in multiculturalist terms in its report 'Ethnic minorities', published in 1979. This report stated that migrants had become permanent minorities becoming what was now an 'open, multi-ethnic society' (ibid.: XX). It argued that:

the assumption that minorities would remain only temporarily in our country has proven to be wrong [...] [and] policy should take into account the possibility of permanent residence in the Netherlands, $[\ldots]$ accepting the fact that in Dutch society ethnic and racial diversity have increased permanently. (ibid.: XXXIX)

Like ACOM, WRR would play an important role in the social construction of minorities and advocacy for a general minorities policy. It stressed the 'common and shared nature' of the problems that ethnic minorities faced, especially 'social deprivation, maintaining their own cultural identity and contact with a different kind of society' (ibid.: VIII). A specific policy for minorities would be required as 'the distinctive nature of [their] problems lies in the fact that many people belonging to ethnic minorities experience them cumulatively; such an accumulation does not apply in the case of members of other economically weak groups in society' (ibid.: VIII). Unlike ACOM, WRR did not elaborate a definition of 'ethnic minorities'. It followed a more pragmatic approach, focusing only on those immigrant groups that had already been the object of government intervention (ibid.: 7). This included the Moluccans, Surinamese, Antilleans and Arubans, as well as foreign workers.

The policy slogan 'Integration with retention of cultural identity' was explicitly denounced by WRR, which believed it would divert attention away from the integration of immigrants in society. The goal instead would be to achieve 'cultural equality' within a multi-ethnic society (ibid.: XX). Without resorting to cultural relativism, WRR adopted a more dynamic or interactionist view of immigrant cultural identities, stressing the need for mutual adaptation.

The question of separate identity needs to be considered in the light of a plural society, in which the majority and minorities are receptive towards one another's views and take account of the essential cultural values of each other's cultures, while at the same time acknowledging that active participation in society by minorities will require a change in attitudes on both sides. (ibid.: XXXVI)

In this context, WRR also clearly posits boundaries to its multiculturalist perspective. Immigrants would have to respect the rule of law and, in cases of cultural confrontations where no compromise seemed possible, the 
council saw no option other than to protect 'the achievements of our culture' (ibid.: XXII).

Besides mutual adaptation in terms of identity development, WRR emphasised the importance of social-economic participation as a condition for the emancipation of members from minority groups. It elaborated a causal theory, which stressed that 'the positive enjoyment and development of a separate culture in freedom would only become possible if a number of basic living requirements are adequately met' (ibid.: XXVI-XIX). Priority should therefore be given to combating social-economic deprivation. 'An active policy to combat social deprivation should [...] be seen as a necessary condition both for the minorities' desire to preserve their own culture in an atmosphere of freedom and for the majority's idea of equality in a multicultural society' (ibid.: XX). An amelioration of the social position of minorities would contribute to 'tolerance of minority cultures by the cultural majority, as the image formed by the majority of a minority and its culture proves to be related to the social status of the bearers of that culture' (ibid.: XX).

\subsubsection{Government departments}

Various government departments were involved in the domain of immigrant integration during this period. However, they framed immigrant integration in different ways. Firstly, various departments were still involved in the differentialist policies targeted at specific migrant groups. This institutional fragmentation reflected the differentialist problem framing that these departments carried until well into the 1970s. It stressed the specificity of the problems experienced by each group, rather than some common denominator to be found across these groups. In accordance with the differentialist perspective, there was no general immigrant integration policy up until this point. Groups were also not bundled under a single generic category ('minorities') but rather, listed specifically by their national origin (Turks, Moroccans, Surinamese, Antilleans). The underlying theory here was that each minority group had a unique position, thus requiring a group-specific approach. Concrete elaboration of this purpose would depend on the group's particular circumstances and characteristics. Foreign workers thus fell under the responsibility of the department that coordinated labour affairs (the Department of Social Affairs), Surinamese and Antilleans fell under the responsibility of the department that coordinated the organisation of welfare to cultural groups (CRM) and refugees were covered by the department that coordinated international affairs (the Department of Foreign Affairs).

The normative premise of this differentialist frame seems to have been especially pronounced: that the Netherlands was not and should not be a country of immigration. This was sustained by economic arguments 
(migrants as a temporary labour reservoir), concerns about the international division of labour (brain drain in countries of origin) and demographic arguments (the Netherlands as an overpopulated country). ${ }^{8}$ The foreign workers memorandum of 1970, developed by the Departments of Social Affairs and CRM, stated clearly that:

It must not be forgotten that the demographic situation in the Netherlands is not such that there would be any reason to promote immigration [...] The Netherlands is definitely not a country of immigration. With every understanding for its human aspects, one cannot conclude otherwise than that our country needs labour power from other countries nor new families from other countries. ${ }^{9}$

Actors framed the problem of immigrant integration in a way that stressed the allegedly temporary character of migration and immigrant settlement. Although the term 'integration' was used in this period in the policy slogan 'Integration with retention of identity', it did not imply permanent integration in Dutch society but rather, social-economic integration as one facet of the so-called 'two-tracks policies' in operation at this time. The other 'track' involved preservation of group structures and cultural identities to help facilitate return migration. Rather than interpreting prolonged immigrant residence as an indication of the settlement of minorities, it was seen as an indication of the need to step up efforts to stimulate return migration. In fact, there were fierce political debates in the 1970s about financial incentives for return migrants, which were cynically labelled 'get-lost bonuses' (Entzinger 1984: 89). Projects were also elaborated with the Department of Development Aid to stimulate return migration with a view to furthering growth in countries of origin through the REMPLOD endeavour.

However, not all government departments supported this problem framing in the second half of the 1970s. CRM, for example, seems to have adopted a somewhat different frame whereby the presence of migrants was seen through the lens of their social position in Dutch society. Prior, in the late 1960s, this department had begun paying more attention to foreign workers as a permanent phenomenon in Dutch society, in response to, among other events, the Twente riots between foreign workers and natives (Groenendijk 1990a: 48; Lucassen \& Köbben 1992). However, this involved recognising the permanency of temporary labour migration rather than the permanent status of migrants themselves. This perspective appears to have changed in the second half of the 1970s, as indicated by CRM's renaming and upgrading of its department on migrant groups to a higher level directorate on cultural minorities (Penninx 1988a: 20). Not only did upgrading to a directorate suggest the issue had gained greater impetus, but the new nomenclature also defined migrants' presence as permanent, 
unlike previous forms of social classification that stressed their foreign or temporary status.

Furthermore, terrorist acts by Moluccan migrants in the 1970s triggered a change in the institutional embedding of policies towards this group. In 1976, the coordination of policies relating to Moluccans shifted to the Department of Justice; in doing so, the matter acquired higher political status, as the Minister of Justice was also Deputy Prime Minister. However, it also implied a 'law-and-order' attitude towards the matter at hand (Entzinger 1984: 107). In 1977, policy coordination shifted towards the Department of Home Affairs, as the Minister of Home Affairs became Deputy Prime Minister in a new government coalition. This switch signalled the rise of a governance approach to Moluccans, which would provide the basis for more extensive change in government policies towards minorities.

\subsubsection{A political entrepreneur}

One political entrepreneur in particular, Molleman, would play a central role in the field of policymaking during this period. Political parties only began to formulate their positions on an immigrant integration policy in the early 1980s (Fermin 1997: 77). The Liberal Party and the Christian Democratic Party made no mention of 'minorities' in their election manifestos until 1981 (ibid.: 84, 121). Only the Social Democrats, one of the three largest parties in Dutch politics, had paid any attention to this issue. This was seen in a special committee on cultural minorities (ibid.: 101) established in 1977 and led by Molleman, a young parliamentarian who became a spokesperson for cultural minorities.

In the same year, during parliamentary debates on the latest instances of Moluccan terrorism, Molleman had called for a more all-encompassing approach to cultural minorities (Molleman 1978; Van Kuik 1986). An interdepartmental committee formed to revise Moluccans policy advised the Minister of Home Affairs (then responsible for Moluccans) that measures taken with regard to this specific group within various government departments should also apply to other minority groups (Van Kuik 1986: 118). However, the minister declined on the grounds of limited administrative capacity. In 1978, Parliament discussed a new government memorandum that revised government policies towards Moluccans, ${ }^{10}$ and Molleman issued a parliamentary motion in which he called for a revision of government policy towards all 'ethnic minorities'. ${ }^{11} \mathrm{He}$ called for a 'coherent set of measures concerning education, housing, employment, health and social-cultural welfare, not just for Moluccans, but for all ethnic and cultural minorities' (Koolen \& Tempelman 2003: 14). Although Molleman was forced to withdraw his motion because of formal objections from his Social Democratic Party, the Deputy Prime Minister/Minister of Home 
Affairs, who was responsible for coordinating Moluccan policy, decided to adopt the motion. Subsequently, Molleman was invited by the minister to take charge of the development of the Minorities Policy Directorate within the Department of Home Affairs. ${ }^{12}$

An article by Molleman (1978) provides important indications of how he framed immigrant integration. ${ }^{13} \mathrm{He}$ rejects the policy slogan 'Integration with retention of identity' on the grounds that the image it portrays of cultures is too static and that it ignores the dilemmas that can be associated with retaining one's identity when migrants prove to be permanent settlers (ibid.: 33). Rather, he calls the Netherlands a 'multi-ethnic society', and adopts an 'interactionist' perspective on immigrant integration, which involves a 'process of ongoing interaction in a network of [...] social relations between groups in a receiving country' (ibid.: 334). He defines migrants as cultural groups, or 'cultural minorities'. This added a crucial argument to the extension of the new approach towards not only the Moluccan group in Dutch society, but also other minority groups, such as Surinamese, Antilleans and foreign workers. ${ }^{14}$

Molleman further argues that, in addition to general measures that would affect minorities, a specific approach to minority groups was required. He refers to policy measures to ameliorate the position of these specific groups as well as the need to promote the 'emancipation' of these groups. In the aforementioned parliamentary motion, called for is a structure of representation and consultation with migrant groups themselves. Finally, being a Social Democrat, Molleman (1978: 328) attributes the most importance to the social-economic deprivation of migrants, and takes a normative position on the transformation of Dutch society into a multi-ethnic society. According to Molleman (1978: 335): 'We must learn to share our historical claims to living on our territory with groups whose cultures deviate sharply from ours [...] We must learn to accept them, as they are, as people with their own cultures'.

This focus on cultural minorities, the call for specific group measures and a normative perspective on a multi-ethnic society indicate that Molleman's was a multiculturalist frame. However, an emphasis on the interaction between groups and prioritising an amelioration of the position of minorities in social-economic domains reveals that his frame also contained some universalist traits. This frame was further developed in the reply memorandum to the 1979 WRR report, the draft minorities memorandum and the final minorities memorandum, all of which were developed under Molleman's directorship of the Minorities Policy Directorate. The reply memorandum explicitly stated that the Netherlands had become a 'multi-ethnic and multicultural society'. ${ }^{15}$ Assimilationism as well as segregationism (or differentialism) were explicitly discarded as models for a minorities policy. 
The acceptance of ethnic minorities as equals excludes the possibility that they are forced to adopt Dutch culture [...]. [O]n the other side, if the term 'society' is to have any meaning, being separated from each other [segregationism] must also be denounced. ${ }^{16}$

Instead, the policy goal was formulated as 'mutual adaptation in a multicultural society with equal opportunities for autochthonous [natives] and allochthonous'. ${ }^{17}$ Also, the reply memorandum concurred with WRR, stressing the need to ameliorate the social position of minorities as the primary condition for integration. ${ }^{18}$ A cultural relativist position is avoided by acknowledging that in some cases 'minorities will not escape adaptation to Dutch society', especially in situations where the freedom of individuals is involved. $^{19}$

These contours would remain largely intact in the 1981 draft minorities memorandum and 1983's final minorities memorandum, despite elaborate and lengthy consultations with various organisations involved in this issue domain (Urbanus 1983). The aim of this minorities policy was to 'achieve a society in which the members of minority groups that reside in the Netherlands can each, individually as well as group-wise, enjoy an equal position and full opportunities for development' (Urbanus 1983: 12). A shift in focus, towards a more group-specific approach, did occur in the draft minorities memorandum, demonstrating a move from universalism to multiculturalism. More so than in the reply, the draft minorities memorandum stressed that group identities and structures can help migrants acquire a stronger position in Dutch society.

It must be seen in a positive light how a migrant in a situation that is both new and uncertain will be committed to maintaining his norms, values and certainties and will search for support primarily from companions. ${ }^{20}$

However, in the final minorities memorandum, the government seems to have stepped back from this more multiculturalist stance (Entzinger 1984: 133), warning against group-specific measures that 'failed to represent minorities in general policies on an equal footing with all other inhabitants'. 21

\subsubsection{A political entrepreneur}

Finally, attention must be given to those actors involved in the various welfare and migrant organisations set up to represent the interests of minority groups. On the whole, members of these organisations were not representatives from minority groups, rather they were led by natives acting on behalf of migrants - the so-called fiduciaries or zaakwaarnemers (Köbben 
1983; Tinnemans 1994). These organisations included the Dutch Centre for Foreigners (Nederlands Centrum Buitenlanders, NCB), the Foundation of Surinamese Welfare Organisations (Landelijk Inspraakorgaan van Surinaamse Welzijnsinstellingen, LISW) and the Consultation Body for Welfare of Moluccans (Inspraakorgaan Welzijn Molukkers, IWM). Only the IWM had significant representation from the Moluccan community itself. In addition, the interests of migrants were often represented by general organisations from civil society that showed a special concern for immigrants, such as church foundations. These organisations and their staff also took on the role of fiduciaries. Often they received support in the form of funding from the Department of Culture.

Despite differences between the various welfare organisations established for different groups, they framed immigrant integration in similar terms. Fermin (1997: 168), in an elaborate analysis of documents relating to these welfare organisations from the early 1980s, has shown that these organisations framed immigrant integration in terms of a collective emancipation process that was to take place from within the various groups. In this respect, their framing was distinctly multiculturalist, as they named immigrant integration in terms of collective emancipation of migrant groups defined as cultural minorities. In causal terms, the underlying theory was that social-cultural emancipation is a condition for integration also, for example, in the social-economic sphere. In this theory, group structures (including group organisations) and group identities would fulfil a central role in the integration process. In some respects, this multiculturalist framing also contained some differentialist traits. The collective emancipation strategy emphasised autonomy within a group's own community by reinforcing the unique cultural community and cultural identity (ibid.: 171). Whenever possible, the organisations resisted what they saw as paternalistic government interference in the cultural sphere (ibid.: 175), arguing instead for a more prominent role of group organisations and rejecting the idea of 'integration' per se, as it would mean 'mutual adaptation' between migrant groups and native society (Urbanus 1983: 13). Most organisations held on to the idea of retaining their own cultural identity and some even cherished the prospect of return migration, still very vivid among migrants at this time (ibid.).

These welfare organisations selected and interpreted the problem context somewhat differently than other actors. For instance, they did not take the Moluccan terrorist acts as 'evidence' that a general immigrant integration policy was needed as a way of preventing migrants from committing such acts but rather, the acts were seen as an indication that migrants and their representatives were not being taken seriously. More generally, these organisations did not interpret evidence of ongoing immigration and immigrant settlement as indications that the government should develop an immigrant 
integration policy; instead, they saw the Netherlands becoming a nation of communities that should be given a say in various domains of government.

In sum, research and policymaking in the late 1970s and early 1980s was, at least initially, characterised by a multiplicity of frames. Some actors, including the Department of Social Affairs, most political parties until the early 1980 s and, to some extent also, the welfare organisations framed immigrant integration in a differentialist way. This meant that there should be no integration policy and that differences between cultural groups were to be institutionalised and, according to some, that return migration had to be facilitated. Adopting a more multiculturalist frame were other actors, including CRM in the second half of the 1970s, ACOM, WRR, policy entrepreneur Molleman and, to some extent, welfare organisations. This meant that immigrants were defined as 'ethnic minorities' or 'cultural minorities', whose integration would be achieved via a combination of general and specific measures for certain groups to promote social-cultural emancipation and social-economic participation. It also placed integration in the normative perspective of the transformation of Dutch society into a multi-ethnic or multicultural society.

These frames also involved different ways of selecting and interpreting evidence about the problem context. In a multiculturalist frame, ongoing immigration and absence of return migration on any significant scale were interpreted as evidence that migrants had become permanent minorities. Along these lines, furthermore, the Moluccan terrorist acts became foci of the deplorable social position of minorities and a significant argument for developing a general immigrant integration policy, so as to prevent such events from recurring. In a differentialist frame, however, select evidence was based on demographic and economic arguments and ideas about international relations (e.g. brain drain). Signs of immigrant settlement and ongoing immigration were interpreted as indications that efforts to stimulate return migration had to be stepped up.

\subsection{The construction of a technocratic nexus}

The rise of the Dutch multicultural model of integration appears to have occurred within a setting initially characterised by a multiplicity of frames. How and why did this multiculturalist model become dominant between the period from 1978 to 1983 ? What was the role of research-policy dialogues in this frameshift? And how were these dialogues organised in this defining period for Dutch immigrant integration policy and research? 


\subsubsection{The field of scientific research}

In the field of scientific research, several actors played a key role in the frameshift from differentialism to multiculturalism. Among the most remarkable were ACOM and WRR.

\section{Boundary work and the 'holy fire' of ACOM}

ACOM consisted of a number of researchers that represented almost the entire immigrant integration research domain in this period. ${ }^{22}$ Although attention for immigrant integration was rapidly growing in the 1970s, not least due to increased research funding by CRM (Penninx 1988b: 21), the number of researchers involved in this field remained limited. Because of its central position, ACOM was able to play a major role in the demarcation of the structure of this evolving field while at the same time obtaining a focal position in terms of research-policy relations and particularly with CRM, with which it was associated.

In terms of demarcation, several leading members of ACOM had distinct ideas about what was considered proper 'minorities research'. This demarcation involved a choice for specific research methodologies and, related to this, also a specific ethos. There was a preference for field research methods, inspired by cultural anthropology, in which the researcher became closely involved with immigrants as research objects in order to uncover their inner world. ${ }^{23}$ According to Bovenkerk and Bunk (1983: 67), this involved choosing specific 'methods and techniques for gathering first-hand information, and to the effects of these methods on relations between the researcher and the people who are part of the processes, situations and events being studied'. Standard techniques of sociological research would be inadequate since 'one is confronted with groups of people and social issues and phenomena that cannot be studied with any degree of verisimilitude by means of standardised questionnaires, official documents and reports or by controlled laboratory experiments' (ibid.). Bovenkerk argues that researchers should choose methods in which they 'spend a long period of time with a group of people in everyday situations [although this is] often not the path of least resistance' (ibid.). The approach adopted by Bovenkerk (1978) and others was revealed in a series of studies that tried to retrieve patterns of discrimination by actually putting the researcher in the shoes of immigrants. Van den Berg-Eldering (1978), for example, studied Moroccans by travelling to Morocco to work with families, learning their language and staying in their homes. Penninx adopted a similar approach doing fieldwork in Turkey and North Africa, for which he learnt Turkish and Arabic. ${ }^{24}$

Köbben, a leading anthropologist at this time, describes this as the 'holy fire' that researchers must possess in order to unravel the living world of immigrants, despite possible resistance from the involved groups or from 
government institutes (Köbben 1980). He contrasts this with research that has been conducted 'in haste [...] from a desk [and] with a preference for numbers and tables, $[\ldots]$ the research tradition of a broad group of sociologists' (ibid.: 10). The 'holy fire' approach allows researchers to better perform their societal function of deciphering the world of immigrants and communicating the results to a broader public. It also involves drawing attention to processes of 'minority formation', for instance, due to discrimination.

This methodological preference was related to an ethos that required researchers to identify themselves with immigrants, who were often defined as the 'underdog'. ${ }^{25}$ For instance, the researcher's ethic would dictate uncovering patterns of racism or discrimination, regardless of opposition from involved parties or criticism from other researchers. ${ }^{26}$ This was manifest in the strong anti-racist and anti-discrimination norm characterising scientific research in this period. ${ }^{27}$ In fact, many studies were oriented towards uncovering patterns of racism. A consequence of such focus, however, was that research in this domain showed signs of being 'fixated' on cultural differences. $^{28}$

This demarcation of immigrant integration research was related to the prominent role of anthropologists in this field (Entzinger 1981: 106). Anthropologists such as Köbben, Bovenkerk and Penninx were among the first to engage in research in this domain and later obtained central positions in organisations such as ACOM. Previously involved in the study of cultures abroad, in places such as the former Dutch East Indies, anthropologists now turned their attention to immigrant minority cultures in the Netherlands (Bovenkerk \& Brunt 1983: 67). This explains why some of their methods (field research) and their ethos (engaging in 'alien' cultures) inspired minorities research in this early period and, according to some, why there was such a strong fixation on the cultural factors of immigrant integration (Choenni 1987).

In terms of coordination, some of ACOM's leading experts advocated that researchers deeply engage with ongoing policy developments. Penninx, one of the researchers closely involved in developing the minorities policy, had a background in social activism, including participation in the Action Group for Foreign Workers, which at this time still advocated a critical, Marxist perspective on immigration. ${ }^{29}$ Penninx was part of a group who believed that scientific research should have a societal function. Anthropology, they said, was useful for the transformation of society. ${ }^{30}$ Their activism concentrated mainly on the housing of foreign workers and language courses. According to Penninx (in De Hart \& Prins 2005: 183), this type of activism was typical for the social sciences in the 1970s. Köbben revealed his social engagement by participating in government commissions on the status of the Dutch Antilles and Aruba and the position of Moluccans in the Netherlands. In this context, he also reflected on 
the difficulties of such political engagement, recognising that politicians would be unwilling to accept unwelcome messages (Bovenkerk, Buijs \& Tromp 1990; Köbben \& Tromp 1999).

This policy orientation was also manifest in how ACOM interpreted its formal role as an advisory body on research programming and policy development for CRM. Already in the form of the 1979 report 'Minorities research advice', ACOM had clearly extended its advisory role into CRM's domain. ACOM (1979: 3) believed that 'in anticipation of developments in the direction of a more integral approach', it should draw in 'many other policy areas where specific mechanisms currently affect the position of [minorities]'. In this period, ACOM openly assumed an advocacy role for the development of a general minorities policy. Furthermore, it established criteria for research priorities that would ensure that the supported research would provide relevant insights into policy development. ACOM tried to ensure policy relevance by establishing criteria for research prioritisation in its publication. These criteria indicated that research should be of 'social relevance', which meant that researchers had to concentrate on the most important questions concerning the position of minorities that were on the agenda (ACOM 1979: 70-72). In addition, research should be 'practically feasible', with a preference for 'large numbers of small studies that do not take relatively long periods of time, rather than a limited number of long research projects' (ibid). Finally, research should focus primarily on those themes that, up until that point, had received little attention.

Thus, the boundary work of ACOM appears to have involved a habitus of engagement. It was twofold: being committed to social issues in order to better the position of minorities (guided by an anthropological ethos and methods) and staying engaged with ongoing policy developments.

\section{Between advising and informing: WRR's boundary work}

Although WRR is not devoted to the field of immigrant integration, per se, it did play a role in the relationship between research and policy in this period. Its first report on immigrant integration in 1979 would mark the beginning of a 'tradition of involvement' in this domain (Meurs \& Broeders 2001) that would award it great authority in this research field in particular.

WRR's boundary work was closely related to that of ACOM. WRR coordinated the development of its report with ACOM in various ways. It consulted ACOM from an early stage in the process of discussing whether or not to formulate a report on immigrant integration for the government. ${ }^{31}$ Subsequently, the project began by commissioning a preparatory study on the state of the art in immigrant integration literature, with which ACOM was again closely involved. ${ }^{32}$ For the study, WRR consulted with Köbben and Van Amersfoort, the two prominent ACOM members. ${ }^{33}$ They, in turn, recommended Penninx, who had also been a member of ACOM since its 
inception. Penninx (1979: 8) based his research primarily on prior studies and interviews with researchers who were involved with ACOM. Furthermore, ACOM members were invited on several occasions to attend council meetings and discuss draft texts of the WRR report. ${ }^{34}$ These points of contact also served to avoid overlap between ACOM and WRR, under the agreement that ACOM would concentrate on coordinating research on the position of minorities and WRR would concentrate on the relations between minorities and Dutch society at large in the context of developing the minorities policy. ${ }^{35}$ The final WRR report stated that 'close contact was maintained with the Advisory Committee in the preparation of this report'. Referring to ACOM's advisory report, which was published in the same week as the WRR report, ${ }^{36}$ WRR stated it is reasonable to expect that the orientation of this report and the research programme will dovetail with one another' (ibid.).

In this way, WRR incorporated the demarcation of proper minorities research by ACOM, including its habitus of engagement. Moreover, two WRR staff members who played an important role in this WRR initiative had been previously engaged in this issue domain. Both had been involved with immigrant groups in their former careers as civil servants. ${ }^{37}$ One of them had been the head of a directorate for foreign workers at the Department of Social Affairs. The fact that this person was now working on a report that would provide the foundation for a general minorities policy, something that had been heavily opposed by the Department of Social Affairs, stresses the frameshift that had taken place within WRR. Both staff members had maintained an interest in this issue and both believed that the events that had taken place in the late 1970s signalled a breakthrough. Moreover, they believed that WRR could have an input in these matters. ${ }^{38}$

In terms of coordinating science-policy relations, WRR exhibited a style of policy engagement similar to that adopted by ACOM. Although it stressed its independence and scientific authority, it also attempted to stay 'close' to the administration so as to be relevant to the ongoing policy developments - keeping the administration close but out. In fact, it was ACOM that encouraged WRR to take up this issue in an effort to reinforce its claims for a general minorities policy. WRR's scientific and political capital - 'its central position and expertise' - would make the argument for a minorities policy more convincing. ${ }^{39}$ Although the WRR council did indeed decide to take up this issue in a report to the government, ${ }^{40}$ some council members were hesitant about the need for the frameshift being advocated by ACOM.

Chairman of ACOM: [...] [I]t is very necessary that in addition to ACOM, another institute engage itself with the minorities issue. The departmental research organisations are less suitable to this aim. WRR could be the proper institute for this, because of its 
integrative and long-term tasks. In addition, WRR is centrally located and enjoys a certain prestige. ${ }^{41}$

The Secretary of ACOM 'refers in this respect to the poor coordination on the part of the government'.

WRR Council member: '[I] am still not convinced that in Dutch government policy a framework would have to be developed for a minorities policy.'

Chairman of ACOM: '[I] consider this necessary, although I could make comments on the feasibility of this problem.'

Penninx: '[I] think that a policy must be developed for all ethnic minorities, so as to guarantee that all groups be offered equal opportunities.' 42

WRR's engagement with the development of a minorities policy was also manifest in the timing of the report in relation to ongoing policy developments. WRR had taken up this issue at a time when the government had already accepted the need for a minorities policy and had set in motion the process of developing such a policy by establishing a Minorities Policy Directorate with Molleman at the helm. To be of relevance to these ongoing developments, WRR tried to advise more rapidly than usual, in just over one year. It also maintained contacts with Molleman, who received a draft text of the WRR report before it was published. ${ }^{43}$ In fact, WRR explicitly oriented itself around these ongoing policy developments at an early stage of developing its report, indicating that its goal was to reflect on policy aims in the context of ongoing changes in the perception of the position of minorities.

The desirability of a general policy for ethnic minorities has already been recognised by many. [...] Also in policy, a - modest - shift in focus towards an integration aim can be discerned. This new realisation demands reflection on the official aims of policy [...] in the context of permanent residence in our society. ${ }^{44}$

Furthermore, WRR's coordination of relations with policy was characterised by a strong belief that by providing policy-relevant knowledge and expertise, it could contribute to the rational resolution of this social problem. ${ }^{45}$ There was a belief within WRR that the social problem of immigrant integration could be effectively resolved if the government, informed by its advice, could bring immigration to a halt and adopt a variety of social measures to promote social-economic participation and social-cultural 
emancipation. This positivist belief in the feasibility of the social problem was also manifest in WRR reports on other contemporaneous social issues. According to one of the staff members involved in WRR's 'Ethnic minorities' report from 1979:

This report fits very well in its epoch. [...] There was still the idea that, in terms of social positions and social deprivation, a lot could be done. That optimism of WRR can be found in many reports from this era. [...] We had the feeling that it was a very clever report, stopping immigration, and step-by-step integrating minorities in Dutch society, and then [resulting in] their social position improving, and the problem being resolved. ${ }^{46}$

The boundary work of engaging with ongoing policy developments was related to concerns about the positioning of WRR. At this time, WRR was still a relatively young organisation (the legislation on WRR was passed in 1976). The second council, inaugurated in 1978, would prove to be especially ambitious in terms of establishing WRR's position. ${ }^{47}$ In this context, it wanted to take hold of the issue, showing how WRR could really 'matter' as an institute that could deal with complex social issues and provide useful policy advice to government. ${ }^{48}$

Efforts to position WRR not just in the field of research, but also in the field of politics are also revealed in a boundary struggle that emerged during creation of WRR's 1979 report. The struggle concerned the issue of whether the role of WRR was to provide 'scientifically sound information' or to provide 'scientific policy advice'. ${ }^{49}$ Fears of an overly technocratic WRR had formally restricted the organisation's role in the 1970s to providing 'scientifically sound information' only. This raised the question of whether or not WRR should add its own analysis to the preparatory study by Penninx. The preparatory study was considered to be of such 'quality' and 'significance' that the council decided to publish this study together with the WRR's report to the government (WRR 1979: VII). This exceptional formula had never been used before.

On one side, there were proponents for providing an advisory report to the government in which the council could formulate its own position based on Penninx's preparatory study and put forward specific policy recommendations. Arguments were made stating 'it is the task of WRR to provide advice, to deliver publications that are of use for policy organisations', 'it is important that the council test its own competencies' and 'a problem-exploration would risk running into the same water as ACOM'. 50 On the other side, there were proponents for a more explorative 'problem orientation', in which WRR would only publish Penninx's preparatory study or expand it with only a brief informative WRR report. This was supported by arguments that providing advice would be 'too risky and 
suggestive [...] as it is no longer entirely based on available information [that] WRR has an informative role and that its advisory task is still contested', and that publication of the preparatory study 'is perhaps not of too much use for government, but it can be of significant importance for society, which is also a task of WRR'. ${ }^{51}$

The outcome of this struggle was decided in favour of the advisory alternative exhibiting a deep policy engagement. WRR wanted to show that it could really matter by exploring the limits of its advisory role and 'testing [its] working methods, and [its] abilities to advise on the short term'. ${ }^{52}$ In addition, the council considered itself obligated to add its own position to the preparatory study 'because of the nature of the study - a policy description and evaluation' (WRR 1989: VII). The expectation was that most people would actually read the WRR report and then selectively read parts of the preparatory study à la carte. ${ }^{53}$ The choice for the advisory alternative was also made in the hope that WRR would exploit the policy window of opportunity that had opened up in this period, with policy in a deadlock after having conceded that a minorities policy was needed in 1978 following the Molleman motion.

It seems possible to develop a proper recommendation within a reasonably short period. The first variant [...] will be taken as a point of departure for this recommendation. It is, however, still possible that in doing so, WRR will trigger irritation from the policy departments. We are, however, faced with a situation where policy is currently in a deadlock. As a result of the public debate that will follow our study [...], there is a possibility that policy priorities can be changed. $^{54}$

The project on ethnic minorities marked a broader change in WRR's role. Whereas in the 1970s, WRR consented to a model of 'moderate decisionism', one in which it provided scientific information as input to political decision-making (Hirsch-Ballin 1979), the project on minorities appears to have been a precursor for a change in WRR's role to provide more normative policy advice. ${ }^{55}$ In this context, WRR's approach shifted from 'a broad approach in which many different topics of government policy for the long-term were dealt with [...] towards an in-depth approach in which only several, essential aspects of government policy are examined [...]' (WRR 1992: 140; author's emphasis).

In sum, ACOM and WRR demarcated and coordinated research-policy relations in rather similar ways. The demarcation of minorities research involved a habitus of engagement with minorities as ethnic or cultural groups who experienced specific problems and, to some extent, a certain cultural fixation. This was influenced by the prominent role of anthropologists in the evolution of this research field, who now held central positions within 
the research field - ACOM, in particular - and had distinct preferences in terms of research methods and ethos. The coordination of relations with policy also revealed a habitus of engagement in both ACOM and WRR. Both were strongly oriented towards ongoing developments concerning government plans for a minorities policy, and they also coordinated their efforts to influence these developments. However, this policy engagement had different structural origins for ACOM and WRR. For the former, this way of coordinating policy relations was related to how it demarcated minorities research in terms of engagement with cultural minorities, something which motivated researchers to engage with policy developments that would affect this position. For WRR, it was rather related to its positioning in the broader context of research and politics in the Netherlands. In fact, producing the 1979 report was part of a repositioning of WRR towards a more policy-oriented advisory role, beyond its formal role of merely providing scientifically sound information.

\subsubsection{The field of policymaking}

The preceding section's analysis revealed the variety of actors involved in research-policy relations during this formative period of the Dutch multicultural model. This section looks into what role the actors played in constructing a research-policy nexus.

\section{The boundary work of no immigrant integration research: Government departments and welfare organisations until the 1970 s}

The relatively late development of immigrant integration research was not only due to an absence of interest in this issue on the part of researchers, but also to the lack of government demand and funding for such research (Penninx 1988b). Although the Department of Social Affairs was responsible for foreign workers, being one of the largest categories of migrants, it was hardly involved in encouraging research into the position of these groups. This lack of interest stemmed from the department's position, which took a labour market perspective on immigration. From this position, it framed immigrant integration in differentialist terms, focusing on the buffer function of foreign workers as a reserve in times of labour shortages. This voided the need for systematic research into the social position of migrants in Dutch society, as these migrants were expected eventually to return to their home countries. In fact, one of the few research projects in which it was involved was REMPLOD (together with several other departments), which explored the prospects of engaging migrants in development of their home countries.

Most research funding in the late 1960s and 1970s came from CRM, which was responsible for providing facilities to migrants during their residence in the Netherlands, for example, in the spheres of housing and 
welfare (ibid.: 19). Although this department did not engage in systematic research programming at that time, it did finance various studies on the social position of migrants (ibid.: 18). For instance, it commissioned a 1971 study on the position of migrant groups by the late Hilda Verwey-Jonker the inspiration for the eponymous institute - joined by a group of researchers including Van Amersfoort and Van Praag.

Furthermore, welfare and migrant organisations were known, on occasion, to have prevented research on their groups. A case in point is the Commissioner's Office for Welfare of Moluccans (Commissariat van Ambonezenzorg, CAZ), which averted research among Moluccans for a considerable time (Penninx 1988b: 17). This discouraging attitude towards research was related to the perceived threat that research would pose to the structural position of these organisations. In particular, research on minorities would form an alternative means for formulating government measures relating to these groups, without the involvement of the communities themselves (Penninx 1988b: 47). In this respect, the relation between welfare and migrant organisations and research bodies, like ACOM, was tense at times.

The absence of steady research interest into the position of migrants must also be considered a consequence of a distinct sort of boundary work during this period. This involved demarcating research and policy in such a way that themes not on the policy agenda were ignored or even discouraged. The absence of a common understanding was of great relevance for government policy of the social position of migrant groups. Based on its structural position and its differentialist framing, the Department of Social Affairs had no interest in researching minorities; rather, it sought research concerning return migration. Welfare and migrant organisations also had no interest in researching minority positions, because doing so could interfere with their structural positions as representatives or fiduciaries of migrant groups vis-à-vis the government.

This way of demarcating research and policy was related to the coordination of mutual relations. Indications of this are provided by the few occasions when the actors were confronted with research on the social positions of immigrants. When CRM commissioned a study by Hilda Verwey-Jonker and enlisted a group to research the position of various migrant groups, controversy emerged over use of 'immigrant' in both the title and actual text of their report. The term would suggest that the groups included permanent immigrants, whereas the government had stated clearly in its 1970 memorandum on foreign workers that the Netherlands was not a country of immigration. As a result, the term 'immigrant' and the title 'Immigrants in the Netherlands' were abandoned in favour of the term 'allochthonous' and the title 'Allochthonous in the Netherlands'. According to Van Amersfoort (1984: 138), one of the authors involved in this publication: 
[...] CRM had given us an assignment to produce the book Allochthonous in the Netherlands. However, the suggested title Immigrants in the Netherlands was confronted with insurmountable objections. [...] The government had just [in 1970] declared in its foreign workers memorandum that the Netherlands was not a country of immigration. [...] The Netherlands had many things.... but not 'immigrants', so it was 'allochthonous'.

Furthermore, when Entzinger published an article in a special issue of the journal Beleid \& Maatschappij on tensions between the norm and the fact that the Netherlands is not a country of immigration, he was met with criticism from CRM (Entzinger 1975). Although he wrote this article independently, he was still a civil servant at the Research and Planning Group of CRM, which at that time still held on to the assumption of temporary migration. ${ }^{56}$ This shows how the boundary work of these actors, aimed at ignoring if not preventing research on minorities, was clearly related to the position of these departments in the prevailing two-track policies and the government's reluctance to concede to being a country of immigration. In the case of migrant organisations, the boundary work was related to their position as representatives for these migrant groups.

\section{Missionary boundary work: The Department of Culture, Recreation and Social Work (CRM)}

As described above, the position of CRM changed during the 1970s. This was due, in part, to developments in the groups that fell under its responsibility - the Moluccans and the Surinamese. ${ }^{57}$ Furthermore, this department was confronted with the norm versus fact issue as far as the Netherlands not being a country of immigration, as a result of its responsibility for providing welfare facilities to the growing number of temporary migrants (Penninx 1988b: 20). As such, change was catalysed not only in how this department framed the issue of immigrant integration, but also in its boundary work practices in the emerging field of immigrant integration research.

CRM altered its discourse on, and relations with, immigrant integration research during the 1970s. By the early part of this decade, the department had formed a Research and Planning Group that would pay significant attention to issues of immigrant integration. This group was to be the main source of immigrant research in this period. It also provided a cradle for researchers (e.g. Van Praag and Entzinger) who would play an essential role in this research domain for decades to come. Furthermore, since 1976, the department was advised on an informal basis by a group of minorities researchers (Rath 1991: 172). The change in discourse not only involved a change in mutual relations, but also in the demarcation of research and policy. For the first time, the department showed systematic interest in 
research on cultural minorities. In 1978, what was once an informal network became formalised through ACOM's establishment. Within this boundary organisation, CRM created a boundary object that, on one hand, demarcated its specific interest for research into minorities within Dutch society and, on the other, coordinated relations between research and policy in terms of the acquisition of more systematic knowledge for the development of government policies towards the minorities.

In terms of demarcation, CRM now showed a specific, systematic interest in acquiring knowledge and expertise on cultural minorities. It now explicitly sought knowledge about migrants as permanent minorities. Furthermore, there was a demand for policy-relevant knowledge to develop policies for these groups. ACOM was to promote 'coherence and accumulation of knowledge for policy formulation and implementation' (Penninx 1988b: 21). It was not an aim to encourage fundamental research. During ACOM's instatement, the CRM minister stated that 'pure scientific research must be considered a luxury that we can hardly afford' (see Van Putten 1990: 359). Instead, research was to provide instrumental insights for policy developments. 'Knowledge on the position of the various categories of cultural minorities' would in itself be 'insufficient'. There was a specific need for 'studies and reports of high quality that must direct, stimulate, support or - when necessary - correct government policy' (ibid.).

This way of demarcating a specific demand for research that provides policy-relevant insights into the position of minorities was closely related to ACOM's own desire to coordinate research and ongoing policy developments. CRM's boundary work involved a 'political manoeuvre' to gain legitimacy for its new policy ideas. ${ }^{58}$ After all, CRM was a relatively weak department that had little means or power to convince other departments that a minorities policy was necessary. It consequently had to shop for alternative venues to propagate its new frame and to overcome the negative feedback from persisting iron triangles. Stimulating scientific research about minorities by founding ACOM in 1978 was, according to Entzinger (1981: 111) - its first secretary, a former civil servant in CRM and future key figure in this research field - a sort of 'missionary project' by the department. Research paved a path for CRM to 'convince other ministries of the necessity for a policy on "cultural minorities" (ibid.). ${ }^{59}$ This also explains why ACOM was able to extend its role beyond that of an advisory committee on research programming for CRM. The idea that ACOM could support the idea of a general minorities policy encouraged its expansion into other relevant domains, such as labour, housing, healthcare and education (ibid.: 109). ACOM would not constrain itself to advising on research programming and clearly also advised on policy issues (Penninx 1988b: 21-22).

The change in CRM's boundary work practices was certainly related to the changes in its position within the field of policymaking at this time. 
Faced with specific problem developments in the groups that fell under its responsibility and, at the same time, meeting reluctance from other departments to respond to these developments, CRM developed a specific interest for policy-relevant research into cultural minorities (demarcation) as a way of convincing other departments of the need for a more systematic approach towards these groups (coordination). This demonstrates a clear connection between CRM's boundary work and the more structural aims of this department.

\section{Politics: Positivism and belief in societal steering}

The Moluccan terrorist acts and the growth of ethnic tensions had created a sense of urgency in Dutch politics about immigrant integration. However, the boundary work practices of political actors in this period reveal that immigrant integration was not defined as a political issue. Rather, research and policy were demarcated in a way that resulted in research gaining a primary role in policy formulation.

This demarcation involved a demand for policy-relevant knowledge and expertise for the development of an immigrant integration policy. Despite the emergent sense of urgency, there was a structural deficiency in terms of knowledge and expertise on how to conduct a policy aimed at immigrant integration. ${ }^{60}$ The government now realised that it had to develop a minorities policy, but lacked the relevant knowledge and expertise to do so. There were few prior experiences with immigrant integration that were considered relevant for this period. For instance, the assimilation approach adopted towards repatriates from the former Dutch East Indies - although often qualified as a 'silent success' (Surie 1971) - was considered unfit for the new minority groups who were of non-Dutch origin. Assimilationism for these new groups was considered inappropriate, whereas for repatriates it was considered suitable since they were defined as returning compatriots. There was also little opportunity for learning from other countries, as the Netherlands was among the first countries to develop a general minorities policy and certainly the first to coordinate it at the level of national government (Penninx, Schoorl \& Van Praag 1993). This sense of urgency, combined with a lack of relevant knowledge and expertise, meant that the government became very susceptible to research findings that could contribute to policy development. ${ }^{61}$

Boundary work defining immigrant integration as a non-political issue was related to specific structural traits of political involvement in policymaking at this time. The positions of the various political parties on immigrant integration were, with the exception of the Social Democratic and extreme-right parties, not well articulated until the early 1980s. In his analysis of the positions of political parties, Fermin (1997: 243) has shown that, although political parties often differed in how they defined immigrant integration, these differences led to minimal open political conflict or 
disagreements. Underscoring this was the fact that Molleman, initially a parliamentarian for the Social Democrats, was asked to direct the development of a minorities policy by Minister of Home Affairs Hans Wiegel, himself a member of the Liberal Party. This political consensus was deemed to have an adverse effect on the rise of extreme-right parties in the late 1970s (Penninx 1988a: 168). Rather than successfully putting immigrant integration on the political agenda, the rise of these anti-immigrant parties seems to have reinforced the political consensus between the main political parties. To prevent parties that would 'play the race card' from benefiting too much, a veritable culture of avoidance was cultivated among the main parties of the political establishment (De Beus 1998; Penninx 2005). What is important is that this culture of avoidance emerged in a period when the public mood was still highly sensitive to alleged discrimination or racism (Tinnemans 1994).

Demarcating immigrant integration as a 'non-political' issue was closely related to the extent to which political actors wanted to be involved in coordinating relations between research and policy. Characteristic of this period was a general positivism and belief in the feasibility of social problems such as immigrant integration. ${ }^{62}$ It was believed that by developing a rational approach towards immigrant integration - bringing further migration to a halt (immigration was still seen as a historically unique event at this time) and adopting rational measures in various domains such as education, labour, housing and welfare - the integration of present groups could be effectively achieved. Thus, an immigrant integration policy would constitute a historically unique effort. ${ }^{63}$ Molleman, head of the Minorities Policy Directorate, argued that immigrant integration should be kept a non-political issue. He believed that a rational approach, based on knowledge and expertise, should be adopted. Furthermore, this consistent policy approach should receive broad political support and be maintained for the length of time necessary to achieve the desired effects, and that it should be free of political conflicts or disagreements. According to Molleman:

It was still not a party-political issue then, and I have always attempted to gain the widest possible support from Parliament. [...] My opinion has always been that this is not a party-political issue. [...] With political arguments you will not be able to achieve broad support for policy in this area. This is a policy that has to be developed and that has to remain for years. And therefore it must not be associated with a particular party so that later on other parties can dissolve it once again. Then a yo-yo effect would be created, that in some cases can be good, but not with this type of policy. ${ }^{64}$

This positivist belief in research's role in developing a rational policy approach was also reflected in the government's emphasis on 'research as an 
instrument for the development of a coordinated minorities policy'. ${ }^{65}$ Significantly, the government called not only for research within the various policy domains and funded by various departments, but also for research of an interdepartmental character. ${ }^{66}$ A budget was made available for such interdepartmental research. Furthermore, this positivist boundary work was manifest in the emphasis the government put into its discourse on the independent and objective status of the 1979 WRR report. In a letter Molleman sent to various actors, which accompanied a number of issues raised by the reply memorandum to the WRR report, great stress was put on the independent status of WRR, a body unrelated to both government and minorities organisations.

WRR is an independent organisation. [...] The government [...] takes great pleasure in the fact that the council has issued its advice on this complex issue. Given the attempts made to develop a bettercoordinated policy on this issue, such a report is very welcome at this moment. Neither government and Parliament nor minorities and minority organisations have been involved in the development and formulation of this WRR report. Also in that perspective, this is an independent report. This makes it possible for minorities, as well as for government and other involved parties, to take complete liberty in sharing their judgements on this report. ${ }^{67}$

According to Van Amersfoort, this way of coordinating relations between research and policy reflected the pillarist tradition of taming controversial topics with instrumental research. In a pillarised society, state policies were to be religiously and socially neutral, so as to be acceptable to all pillars. Technocracy was therefore essential for the functioning of the state: 'the task of politicians was to construct structures in which the religious compromise is established, so as to leave actual policy formulation to experts' and the task of experts was 'to reach technocratic compromise for practical policy problems, which are presented as much as possible as objective, technical solutions' (Van Amersfoort 1984: 122). Although Dutch society had been de-pillarising since the 1960s, it seemed to have reverted to this 'traditional ploy' of technocratic compromise in the case of the minorities policy in the late 1970s and early 1980s (Hoppe 1993; Rath 2001). Immigrant integration was considered 'too hot to handle' for the Dutch political establishment, fearing it could unbalance the fragile coalition system and possibly fall into the hands of extremist parties that could play the race card.

It was common practice not to make a hot issue of something; sensitive subjects were usually resolved by a technocratic compromise. In that process, experts had acquired a dominant, if instrumental, 
role. Hence, a type of social science research had arisen with strong politically-directed traits, and a type of researcher who saw their task primarily as one of 'service to the community'. [...] Particularly in the late 1970s and 1980s, the time when the ethnic policy was still under construction, the common opinion among leading politicians was that immigration and the incorporation of immigrants was too sensitive to make a hot issue of it. So, they reverted to the traditional ploy; resolving the issue by developing a technocratic compromise, in the process of which social scientific researchers acquired a dominant role. (Rath 2001: 150-151)

In fact, this boundary work undertaken by political actors involved a coordination of close relations between researchers and policymakers, rather than between research and politics. The Minister of Home Affairs, who was responsible for the development of the minorities memorandum between 1981 and 1983, even remarked that there had been very little substantial political debate on this memorandum, for instance, within the Council of Ministers.

Issues concerning minorities were always dealt with in the margins of the Council of Ministers during my period as a Minister. [...] It was always in the sphere of procedures and competencies. I cannot recollect substantive debate about the minorities policy within the Cabinet or the Council of Ministers. It may be that, in this, I am doing an injustice to all those civil servants that were already very substantively involved in these issues. (Van Thijn 1985: 5)

There was, then, important differences in the boundary work of actors in the field of policymaking. Specific departments, such as the Department of Social Affairs, as well as welfare and migrant organisations, demarcated and coordinated research-policy relations in a way that either showed little interest for, or even obstructed research about, ethnic minorities. This boundary work was related to the structural positions of these actors who had, respectively, a structural labour market focus on migration or perceived research as a threat to their position as representatives of migrant groups vis-à-vis the government. In contrast, CRM defined research-policy boundaries in terms of active acquisition of policy-relevant knowledge and expertise on minorities that would provide support to the development of a minorities policy. In this respect, by establishing ACOM and promoting research as an instrument for convincing other departments of the need for a minorities policy, CRM seems to have engaged in missionary boundary work. Finally, political actors in this period demarcated immigrant integration as a non-political issue and coordinated research-policy relations in a way that expressed a positivist belief in the contribution of research to the 
development of rational policies and in a way that would avoid political conflicts over this sensitive topic. Thus, this analysis not only reveals important differences in boundary work practices, but also how they related to the different structural positions of these actors in the field of policymaking.

\subsubsection{A technocratic research-policy nexus}

Having analysed how key actors defined research-policy relations, the next step would be to analyse how the boundary work undertaken by actors from the fields of politics and research synergised to produce a specific 'boundary configuration'. In other words, reaching beyond the actor-level, what structure of research-policy dialogues can be identified between research and policy actors?

\section{The convergence of research and policymaking}

There was a very explicit connection between the reports published in 1979 by ACOM and WRR and the development of the minorities policy between 1980 and 1983. First, both ACOM and WRR were explicitly oriented towards influencing policy and advocating a minorities policy. Although the former observed that 'it is not the task of ACOM to develop a policy vision concerning minorities' (ACOM 1979: 3), it clearly went further than advising only on research programming. In fact, several of its reports were 'veiled policy advices'. ${ }^{68}$ Its report 'Minorities research advice' from 1979 included extensive passages on the position of ethnic minorities in the Netherlands (chapter 2); 'government policy in the past, present and future' (chapter 3); and the relevance of the 'cultural orientation of minorities' (chapter 4). The 'Ethnic minorities' report was also highly policy-oriented. In addition to several chapters containing problem analysis, it contains chapters on 'the general contours of a minorities policy' (chapter 4); on 'the contents of a minorities policy in headlines' (chapter 5); and on migration policy (chapter 6). Six out of seven major conclusions directly concerned government policy. Moreover, WRR commented on newly taken policy measures, saying, for instance, it: "positively values the decision to attribute this function [coordinating minister for the minorities policy] to the Department of Home Affairs, as it expresses how this not only concerns social-cultural issues but also other sorts of policy issues' (WRR 1979: XXV).

Molleman and his Minorities Policy Directorate made direct use of these documents for the development of a minorities memorandum. The reports were well timed with the policy developments that had taken place since the parliamentary debates of 1977 and the motion put forward by Molleman that led to establishment of a Minorities Policy Directorate in $1979 .{ }^{69}$ They were taken as a starting point for the formulation of a 
minorities policy. An interdepartmental committee for the coordination of a minorities policy was established to write the reply memorandum to the WRR report. The Council of Ministers established this committee, which included secretary-generals and general directors of various departments, after having received the WRR report. ${ }^{70}$ In writing the reply memorandum, the committee followed the structure of the WRR report, discussing its various conclusions in a series of meetings. ${ }^{71}$ Consequently, the structure of the WRR report was also reflected in the draft of the minorities memorandum. Note how the chapters of the reply memorandum reflected those of the WRR report.

Chapters from the reply memorandum:

1) Immigration and its meaning

2) The situation of minorities

3) Policy assumptions

4) Amelioration of the situation of minorities

5) Means for realising policy

6) Restrictive migration policy

Chapters from the WRR report:

1) Introduction

2) Short sketch of the issue

3) Expected developments

4) Policy assumptions

5) Contents of a minorities policy in headlines

6) Future migration policy

7) Conclusions

There were also immediate relationship between the research and policy actors. Not only was there a close network of personal contacts, there also seems to have been a regular exchange of actors between the fields. An important illustration of this was obvious in the various roles Penninx fulfilled for ACOM and WRR, as well as in development of the minorities memorandum. As one of the founding members of ACOM, Penninx wrote the influential preparatory study for WRR and was also involved as a civil servant at CRM during development of the draft minorities memorandum, having acted upon a request made by Molleman, director of the Minorities Policy Directorate. In addition, Penninx remained an ACOM advisory member on behalf of CRM during the 1980s (Rath 1991: 172), and took office at CRM immediately after completing his study for WRR.

On the same day as I had concluded my preparatory study for WRR, on October the 1st of 1978, I took office at the department $[\mathrm{CRM}]$. So, at the same moment the WRR report itself was 
published, I became a civil servant. After the reply memorandum was published in 1980, a department was established at the Department of Home Affairs for the coordination of the minorities policy. The director of this minorities department then asked me [...] to write the minorities memorandum. In this context, I was hired from the department [of CRM] for the Department of Home Affairs. [...] In this respect, I switched hats from researcher to civil servant. Of course, my name is not at the top of these policy documents. However, he [the director of the minorities department] wanted to really move ahead, now that his new department was established, and wanted to develop a draft memorandum very quickly, and the quickest way to do so was to hire the civil servant who already knew everything about the issue. ${ }^{72}$

There are indications that Penninx's personal relationship with the government was not atypical for research-policy relations at the time. For instance, another pioneer of immigrant integration research, Entzinger, was not only ACOM's first secretary, but had also been a civil servant at CRM. Several other ACOM members had also already conducted research that had been commissioned by CRM (Verwey-Jonker, Van Amersfoort, Köbben). In terms of structural relations, the boundaries between the fields of research and policy were apparently very permeable.

\section{Primacy of scientific research}

Indications show that scientific research found primacy by fostering mutual relations with the policy field. Developments in policymaking were strongly influenced by the ACOM reports and, to a greater extent, those by WRR. In fact, the extent to which WRR's 1979 report was 'silently' and 'directly' taken up in government policy was rather exceptional compared with its other reports (WRR 1987b: 44).

The major impact on policy developments also involved ACOM, which had not only collaborated on this report, but also, more generally, occupied a central position in the field of immigrant integration research. Molleman indicated that his ideas about a general minorities policy were greatly influenced by the works of Entzinger (1975), Van Amersfoort (1974) and various publications by Köbben. Their research convinced Molleman that migrants had become permanent ethnic or cultural minorities and that a different policy approach was needed. ${ }^{73}$ Furthermore, Penninx admits that he 'had never been as influential as he was during the period between 1978 and 1981 ', ${ }^{74}$ when he was not only involved in ACOM and played an important role in the 1979 WRR report, but also helped develop the minorities memorandum in response. It is no surprise, he notes, that the draft minorities memorandum reflected his own preparatory study from 1979 in 
many ways: 'It is difficult to expect that a memorandum you ask me to write now would be very different from something I wrote one and a half years earlier as a researcher. ${ }^{75}$

The primacy of these research actors is also indicated by the concrete way in which the Interdepartmental Committee for the Development of a Minorities Policy responded to these reports. This committee decided that the reports by WRR and ACOM raised such a sense of urgency and addressed such a sensitive issue, that the committee had to announce concrete policy measures in response; simply announcing further research would not suffice.

Because of the urgency and sensitivity of the minorities issue, it seems politically undesirable for the Cabinet to proclaim only further research. The questions that are posed to the Cabinet in the documents in question are already too concrete for this. [...] Unrealistic, too high expectations should not be raised, but neither should the first response give evidence of too much reservation. [...] Announcing concrete measures seems very appropriate. ${ }^{76}$

Molleman was familiar with the recent publications by Entzinger and Van Amersfoort, as well as with some of Köbben's work. He was convinced that migrants had become permanent ethnic or cultural minorities. ${ }^{77}$ Already by 1977, during parliamentary debates on the spate of Moluccan terrorism, Molleman had called for expanding the debate beyond that of a narrow focus on Moluccans (Van Kuik 1986). In that same period, an interdepartmental committee for the revision of the Moluccans policy advised the minister responsible for this group that current measures in place in various departments for the Moluccans should also apply to other minority groups. ${ }^{78}$ However, the minister declined, believing that his administrative capacity would be too limited for this aim. ${ }^{79}$

Thus, the structural configuration of research-policy relations in this period was characterised by convergence between both fields (through direct mutual interaction) and by primacy of research in policy formulation (scientific primacy). Suggested thus is that the research-policy nexus in this period was a technocratic boundary configuration. Indeed, the research-policy relations seem to approximate a model of 'science speaking the truth to power'. This technocratic configuration of mutual relations, however, must be held up against the backdrop of its actors' structural positions. In particular, the configuration was produced by a mutually reinforcing pattern of boundary work by ACOM, WRR, CRM and the Minorities Policy Directorate under Molleman. On one hand, there was the boundary work of ACOM and WRR, which involved demarcating the role of research as producing policy-relevant knowledge about ethnic minorities and coordinating its relation to policy in terms of policy engagement. On the other 
hand, there was CRM's boundary work and Molleman's directorate, which had both missionary and practical needs for policy-relevant knowledge on minorities, and wanted to coordinate research-policy relations in a way that allowed for the development of a minorities policy without politicising the sensitive issue. In this respect, the technocratic way of configuring research-policy relations involved a structural symbiosis between both fields.

\subsection{Technocracy and the rise of the multicultural model}

As we have just seen, research-policy relations from this era are best characterised as the technocratic type of research-policy nexus. Yet, this nexus does not explain precisely why a multiculturalist frame of immigrant integration emerged. It does not account for why a frameshift occurred in the first place, or why, more specifically, the multicultural model emerged, or whether critical reflection about this model took place. Thus, the next step is to analyse the role of the nexus in the research and policy frameshift during this period, its contribution to the rise of multiculturalism in particular and, finally, the extent to which the nexus did or did not contribute to critical frame reflection.

\subsubsection{The structural effects of technocracy}

The technocratic research-policy nexus can also have effects on the structural properties of both the research and the policy fields. In what way, thus, did this nexus affect the position of key actors in both fields? And how did this contribute to a pronounced frameshift during this formative period in the fields?

\section{The structural symbiosis between research and policy}

The technocratic research-policy nexus strengthened the structural position of specific actors in research and policy. In the field of research, it provided a boost to the WRR's institutional authority, which, as a result of its direct significant effect on the development of the minorities policy, was able to show that it could 'matter' as an institute in such complex and sensitive domains. It demonstrated to WRR that a more in-depth approach to specific issues and a stronger orientation on policy advice - instead of merely informing policy - could be successful. Together with other reports from this period, 1979's 'Ethnic minorities' is illustrative of a broader structural change in WRR, whereby it extended itself beyond its moderate decisionist role of the 1970s (as described by Hirsch Ballin (1979)) to a more technocratic role the following decade. This first report marked the beginning of a tradition of WRR involvement specifically in the domain of immigrant integration. 
Furthermore, the technocratic boundary configuration strengthened the position of ACOM. ACOM stayed central in the research-policy nexus and its role in the 'dynamic networking' between researchers and policymakers during this period proved crucial for development of what has been described as the 'minorities research industry' (Essed \& Nimako 2006). Not only did the amount of funding for research coordinated by ACOM increase, but the technocratic boundary configuration also enabled ACOM to be closely involved in policy developments and the societal translation of its research in accordance with its habitus of engagement.

In the field of policymaking, the technocratic nexus also strengthened the position of CRM. The influence of ACOM and, to some extent, WRR in the development of a general minorities policy suggests that this government department's missionary boundary work was successful. Indeed, its intention to use scientific research as a way of convincing other departments of a need for policy aimed at the integration of cultural minorities was realised. However, the coordination of this minorities policy remained outside the responsibility of this department. It was assigned to the Deputy Prime Minister, who was then Minister of Home Affairs and had already been made responsible for the coordination of policy for the Moluccan community. The department was also majorly involved in the interdepartmental elaboration of this policy. As a result, CRM's structural position only partially benefited from the technocratic boundary configuration.

The influence of another actor in the field of policymaking - Molleman and the Minorities Policy Directorate - was more considerable. This technocratic boundary configuration provided the directorate with the required policy-oriented knowledge and expertise for developing a minorities policy. It also enabled Molleman to develop a minorities policy without politicising the issue and without involving the welfare and migrant organisations that were so closely linked to the differentialist policies of the 1970s.

\section{Positive feedback: Breaking the iron triangles of differentialist policies}

The structural symbiosis between the positions of ACOM, WRR, Molleman's directorate and, to some extent, CRM, described by several authors as a 'technocratic symbiosis' (Rath 2001: 153; Van Amersfoort 1984: 132), was a source of positive feedback on the changes advocated by these actors. It strengthened the structural positions of those actors in support of a minorities policy and weakened the position of those who opposed it. The Department of Social Affairs was left out of this symbiosis, although it did remain one of the departments involved in the interdepartmental coordination of the minorities policy. However, eventually this department also changed its differentialist frame towards a more multiculturalist one. In addition, political parties that opposed a minorities policy were forced aside by the technocratic symbiosis. In fact, the role of political parties overall was rather marginal. This was largely because of their still 
minimally articulated positions on immigrant integration, but also because they supported a technocratic approach to policy development as a means of avoiding politicisation that could possibly benefit extreme-right parties.

Furthermore, the technocratic boundary configuration had a negative effect on the structural position of the prevailing welfare and migrant organisations in policymaking. These organisations, together with the Department of Social Affairs, CRM and specific political parties, fulfilled a central role in the differentialist or 'categorical' policies of the 1970s. Now, their role in policy formulation was marginalised by ACOM's and WRR's prominent positions. This is indicated primarily by the marginal role that welfare and migrant organisations played in the development of the minorities memorandum. The process of formulating the memorandum began with the reply to the WRR report, followed by the draft minorities memorandum that was written by Molleman together with Penninx and others. It was only at this stage that welfare and migrant organisations were consulted about their ideas. Even then, their ability to influence changes in this draft text seems to have been limited (Van Kuik 1986). For instance, the final minorities memorandum put less emphasis on collective emancipation as a strategy for integration than the draft minorities memorandum, which was one of the central aspects of the frame of these welfare organisations (Fermin 1997 178). In the media, these organisations are often recorded as claiming that policy was being made 'about' them, not 'with' them. ${ }^{80}$

Molleman was one of the entrepreneurs to break the iron triangles formed by these welfare organisations. From a multiculturalist perspective, he favoured the policy involvement of migrant organisations; however, he believed that this should come out of migrant self-organisation rather than the fiduciary organisations known as zaakwaarnemers that were then dominant. By proposing a general minorities policy for all immigrant communities, Molleman broke from the established structures that advocated a more differentialist approach. In doing so, he was met with significant negative feedback from the actors within.

I issued a motion for developing a minorities policy for all the different groups. I did that without notifying the chairman of our party in any way [...] At one point, the chairman found out and was not at all pleased. The chairman was a CRM man. [...] Also in the area of welfare work, there was an iron triangle: those were civil servants, politics, especially the Social Democratic Party and the Christian Democratic Party that was then being established, and the people in welfare organisations. They all held on to each other. So, what I was doing was breaking into established patterns. [...] [T]he fear was then, and not without cause, that that pattern was to be broken. [...] So, I had to withdraw that motion. But [the Minister of Home Affairs] had already taken notice of the motion and found it 
a good case; he was way ahead of his time. Then he said to me: 'I want to take over your motion anyhow, but you will have to come over and implement it. You'll have to become minorities director and then I will arrange things in the Cabinet so that this is the way it will be done. ${ }^{81}$

The establishment of ACOM as a technical-scientific committee also formed an important factor in terms of generating positive feedback regarding a break with established structures. Whereas advisory bodies for research programming generally involved tripartite bodies in which target groups, scientific experts and government were represented, in this case, the responsibilities of these three actors were clearly separated (Entzinger 1981). In this context, Penninx argues that 'this choice is not derived from experience with research programming but is rather a consequence of a choice for separated responsibilities in the development and implementation of [...] policy' (1988b: 26). This political choice - not to involve ethnic expertise in the technical-scientific ACOM - was related to a certain cynicism concerning the ethnic expertise that these welfare organisations claimed to represent. ${ }^{82}$ As previously noted, these organisations mainly consisted of native Dutchmen who represented the interests of migrants, the so-called zaakwaarnemers. Their expertise was criticised by some as 'white expertise' rather than 'ethnic expertise' (Choenni 1987). In the 1979 WRR report, as well as in the 1980 reply memorandum, ${ }^{83}$ critical remarks were made about the need to enhance the representation of migrant organisations.

At the same time, welfare organisations opposed the plans for a technical scientific ACOM. They put forward practical objections, such as the fact that they had hardly been consulted in the process of establishing ACOM, but also more substantial objections, particularly that ACOM would mainly represent 'white expertise' in contrast to the welfare organisations able to draw upon the 'ethnic expertise' of immigrant groups. Furthermore, they protested that the establishment of ACOM undermined the position of their organisations, which had traditionally had an important role in differentialist policies (Van Putten 1990: 360). ${ }^{84}$ In 1980, when a 'research programming cycle' was set up, minorities organisations were given an advisory role, alongside ACOM. This cycle entailed, firstly, that ACOM and minorities organisations advise on research programming; secondly, that the Interdepartmental Committee on Minorities Research would formulate a draft research programme; thirdly, that the minister ask minorities organisations and ACOM for comments on this research programme; and finally, that the government establish the research programme (Penninx 1988b: 26). In practice, their influence on research programmes was rather limited. 


\subsubsection{The technocracy of multiculturalism}

The technocratic research-policy nexus involved a structural symbiosis between specific research and policy actors that created a powerful actor constellation in favour of a frameshift. But why did this involve a frameshift from differentialism to multiculturalism? Put another way, why did this technocratic symbiosis produce the multicultural model for which the Netherlands would become famous?

\section{The minorities paradigm in research framing}

The technocratic boundary configuration and the active role played by ACOM and WRR in this configuration, appears to have contributed to a paradigmatic status of one particular problem frame in immigrant integration research. ACOM's central position, which was reinforced by this technocratic symbiosis, had significant influence on problem framing in immigrant integration research. This relates to ACOM's influence on research programming, its central position in terms of government and also its exclusive position within this research field. In this context, Penninx (1988b: 37) refers to critiques of ACOM: 'in some circles of researchers ACOM was seen as a biased group of advisors that function as gatekeepers to [...] research funding and attributed no or insufficient attention to specific subjects or disciplines' (1988b: 37).

There are indications that in the late 1970s one frame became dominant, the so-called minorities paradigm, with its heavy-handed multiculturalist approach (Rath 1991). This paradigm framed immigrant integration in terms of the emancipation of ethnic or cultural minorities in social-economic and social-cultural domains by means of a group-specific approach, as well as within the normative perspective of transforming society as a whole into a multi-ethnic society. Rath (2001: 152), following Bovenkerk (1984: 35), observes how

the development of political-economic theory on guest workers in the Netherlands was interrupted quite suddenly, precisely at the moment that the state incorporated researchers into the bureaucratic apparatus and initiated wide-scale funding for politically relevant research.

This is especially striking because of a strong presence of the critical tradition in this field until the 1970s (as evident in the careers of, for instance, Penninx) and also because of the importance of Marxist perspectives in other countries, most notoriously in the UK (Miles 1987). However, this form of race-critical research would prove to be short-lived in the Netherlands (Essed \& Nimako 2006). As a result, the development of a 
technocratic nexus between immigrant integration research and policy coincided with the disappearance of specific knowledge claims.

Researchers claim that ACOM significantly affected immigrant research in the late 1970s and early 1980s. Penninx (1988b: 35) concludes that 'the influence of ACOM in its first phase of existence has been very significant'. Nearly all of the projects that ACOM proposed in its first report to government, the 'Minorities research advice' (1979), were eventually implemented with government funding. Entzinger (1981: 116), then ACOM secretary, concluded that the function of this advice, to 'stimulate research to attribute more attention to the issue of immigrant integration', 'has certainly succeeded'. ACOM would have been 'overwhelmed' with research proposals and claims on particular research themes, while 'the principles, design and goals of the 'Minorities research advice' were rarely or never subject of discussion' (ibid.). Entzinger adds that this lack of debate around the ACOM report may have been caused by researchers' fear of criticising the organisation that would later advise government on whether or not to allocate funding to specific research programmes. Given this relationship, any criticism of ACOM could be detrimental (ibid.: 117).

\section{The logic of minorities in policy framing}

The technocratic boundary configuration seems to have contributed to a dominant logic of problem framing in the field of policymaking. A predominantly multiculturalist problem frame emerged in this field. This involved an emphasis on minorities as the main object of policy. Not only were research actors, such as ACOM and WRR, heavily focused on minorities, but CRM and the Minorities Policy Directorate had a minority focus, too. Although they framed immigrant integration using the normative perspective of Dutch society becoming a multi-ethnic society, the focus was more on minorities as policy objects than considering society at large. By shaping a structural symbiosis between a specific network of actors that shared this primary focus on cultural or ethnic minorities, the technocratic boundary configuration divorced the debate about ethnic minorities from larger debates about the transformation of Dutch society into a multi-ethnic society. It seems to have reinforced the logic of minorities in the framing of immigrant integration policy.

This logic of minorities surfaced within a certain reluctance, if not taboo, though with a clear idea of relating immigrant integration to broader social issues, such as national identity and social cohesion (Tinnemans 1994: 256). The sociologist Vuijsje (1986) attributes this reticence to a post-war sensitivity about discussing ethnic differences. The legacy of World War II created what Vuijsje describes as a vermoorde onschuld ('murdered innocence') in dealing with 'weak' minorities and with cultural differences. At that time, discussing ethnic differences in relation to their influence on native society led all too easily to accusations of discrimination, racism or 
fascism (Tinnemans 1994: 253). The consequence of this was the emergence of a political correctness in dealing with minorities that was to persist for a considerable period (Werdmölder 2003).

This reluctance was reinforced by growing concern about the extreme right and the alleged rise of racism in Dutch society, which appeared to be fostering a strong anti-racist movement in Dutch society and politics. Furthermore, it promoted a particular sensitivity for issues that revealed discrimination, racism or fascism, concepts that were often used interchangeably. A case in point involves public statements made by Couwenberg (1982), a professor of constitutional law, who posed the question: 'How many foreigners can a European cultural people bear without losing its identity? ${ }^{85}$ In this context, he called for a restrictive migration policy in the interest of national demographic and cultural politics, arguing that similar claims made by those such as the extreme right of the Centre Party at least be considered legitimate. These statements triggered broad indignation, leading to accusations that Couwenberg was in fact racist and fascist.

In sum, the technocratic symbiosis contributed to the development of a minorities paradigm in immigrant integration research. In the same period that ACOM and WRR played an active role in shaping the technocratic boundary configuration, this paradigm rose to a hegemonic status while other paradigms (such as critical Marxist and nationalist frames) fell into disuse. Furthermore, it contributed to the consideration of minorities in policy framing. It confined policy development to a limited network of actors that shared a specific focus on minorities and also advocated a specific approach to minorities. Consequently, it divorced the issue of how to achieve the integration of minorities from the larger issue of the transformation of society into a multi-ethnic society. In fact, there seems to have been a certain reluctance, if not taboo, in linking ethnic differences to broader national issues.

\subsubsection{Technocracy and frame reflection?}

Thus, the technocratic research-policy nexus contributed to both the structural and substantive conditions that gave rise to a multicultural model in Dutch immigrant integration policy and research. Yet, did it also contribute to critical reflection on this model? For example, did research-policy dialogues actually involve critical reflection about this model in comparison with alternatives; or did the nexus push this model in different ways?

\section{Technocratic opportunities and constraints for frame reflection}

The technocratic boundary configuration offered some structural opportunities, as well as constraints for a critical dialogue between research and policy. Firstly, it contributed to opening up a first form of debate between 
research and policy on immigrant integration. The boundary work of specific actors played an important role in breaking the iron triangles that held on to a differentialist approach. The positivism and missionary boundary work of certain policy actors (CRM, Molleman) in combination with an engagement in the position of minorities and the involvement of certain research actors (ACOM, WRR) in policy development, created a structural symbiosis between the actors that advocated a different, more multiculturalist approach to immigrant integration. As a result, this technocratic symbiosis became a source of positive feedback on the positions of these actors in their fields. However, the symbiosis also imposed a new constraint on the dialogue between research and policy, as it limited it to the small group of actors involved in the structural symbiosis. For instance, it excluded welfare and migrant organisations, keeping this issue out of open political debate and also seeming to have limited the openness of debate on the research side of the nexus.

Secondly, technocracy contributed to the development of an alternative multiculturalist frame alongside the prevailing differentialist approach. Yet, it simultaneously contributed to the exclusion of other frames. For specific actors, namely CRM and Molleman's directorate, technocracy provided a way of developing an alternative and scientifically legitimate frame that would evade the sensitivity, or even taboos, arising from concerns about the politicisation of this issue. The confrontation between research actors involved in the development of the new multiculturalist frame and those actors that advocated the differentialist approach also led to the articulation of these two models. This was manifest in, for instance, ACOM's and WRR's reports, which clearly addressed the level of problem framing, but also in influential publications such as Van Amersfoort's study (1974), an article by Entzinger (1975) about norms versus facts of not being an immigration country and a study by Bovenkerk (1974b) expressing doubts about return migration. At the same time, technocracy also contributed to the exclusion of alternative models, such as Marxist frames in research and nationalist (extreme-right) frames in politics and combinations of multiculturalist and differentialist frames as advocated by welfare organisations.

Thirdly, and closely related to the second point of selective inclusion and exclusion of frames, is that the technocratic boundary configuration does not appear to have contributed to critical reflection about problem framing. In fact, it seems to have been designed as a strategy for getting the multiculturalist frame on the agenda. Both ACOM and WRR were oriented towards ongoing policy developments in order to advocate one specific (multiculturalist with universalist traits) approach. They made no attempts to foster critical reflection about problem frames; instead, they stimulated diffusion and elaboration of a particular multiculturalist frame. In fact, this analysis has shown that both organisations took this problem frame as a starting point for their involvement in this domain rather than as 
an outcome of reflection. This absence of interest in reflection about alternative frames was also manifest in the practices of policy actors such as CRM and Molleman's Minorities Policy Directorate. Both institutions regarded research as a means (i.e. CRM) of convincing other actors of the need for a more multiculturalist policy approach, or as the source (i.e. Molleman) of practical knowledge and expertise required for developing such a multiculturalist policy approach. Thus, the actors involved in the technocratic symbiosis between research and policy took a multiculturalist frame for granted rather than recognising that they had encouraged critical dialogues about this and other alternative frames.

Fourthly, instead of a pragmatic willingness by actors to adopt their frames in response to reflection, there was a certain reluctance, sensitivity even, in both research and policy to discuss their multiculturalist frames critically. On one hand, there was a social engagement in immigrant integration research with the position of minorities. This was not only expressed in a methodological preference for field research but also in an ethos that urged researchers to put themselves in the shoes of the migrants, with the distinct aim of contributing to an improvement in their position. On the other hand, there were also important normative facets in immigrant integration policymaking that hampered a pragmatic attitude towards problem framing. This created reluctance to discuss ethnic differences vis-àvis broader societal developments.

Finally, technocracy did generate trust within a relatively small network of actors involved in the technocratic symbiosis. However, the other side of the coin seems to have been a distrust of other actors. This included a suspiciousness towards welfare organisations with questionable representation as well as a general distrust of extreme-right parties and politics, with whom potential conflicts could unfold, thus threatening the development of a rational, consistent approach to immigrant integration.

\section{A moment of reflection?}

The role of the technocratic boundary configuration in generating dialogue between research and policy seems to have been rather limited. It was not designed to stimulate critical reflection about problem framing but rather, to generate a structural symbiosis between actors that advocated a multiculturalist framing. To the extent that frame reflection did take place, it primarily involved a confrontation between this multiculturalist approach with the prevailing differentialist model, rather than a systematic analysis of various alternative frames.

Thus, the technocratic boundary configuration was designed to establish the multiculturalist-with-universalist-traits frame rather than to promote critical frame reflection. The 1979 WRR report seems to have played a key role in getting this frame accepted. It provided a synthesis of available knowledge and expertise through coordination with ACOM and others. 
Furthermore, it served as a direct precursor to the reply memorandum, the first to elaborate policy contours, and the final minorities memorandum. This, then, justifies a closer look at whether frame reflection did play a more prominent role within WRR.

Throughout the course of developing the 1979 WRR report, a small but important frameshift was taking place. First drafts of the WRR report adopted a problem frame that was predominantly multiculturalist, or as one of its authors names it, 'interactionist'. ${ }^{86}$ This concerned references in the report to the Netherlands as an 'open, multi-ethnic society' and a discussion of mutual adaptation between minorities and natives. In later drafts, references were added to define the limits of such an interactionist approach. For instance, there were passages on 'elements of other cultures where no or almost no compromise with Western possibility is possible' and those that 'leave no other possibility than to defend the achievements of our culture against contending claims', and about 'the need and space for many adaptations in laws and regulations at government and private institutes to the situation of a multi-ethnic society, without affecting the cultural achievements of our society' (WRR 1979: XXII).

These revisions were a product of a debate within WRR about the interactionist/multiculturalist frame formulated in the first drafts. ${ }^{87}$ The author of those sections of the first drafts relating to Dutch society and cultural diversity advocated a perspective that allowed for open interaction between minorities and natives, without the latter exploiting its dominant position to enforce cultural assimilation. ${ }^{88}$ Thus, this author, a staff member, raised concerns about the asymmetrical relationship between minorities and natives and called for the kind of open, multi-ethnic society that would allow such interaction.

At the same time, several council members raised doubts about an interactionist perspective. In particular, they expressed concerns about the relation between cultural diversity and the rule of law. They argued that the rule of law contained codifications for cultural achievements with which one could not interfere. ${ }^{89}$ For example, one council member worried about the compatibility of Islam with the rule of law and the conflicting values and norms that could arise in this context. ${ }^{90}$

This posed a dilemma for the chairman of the project group: "either the council chooses for interaction based on equality with unlimited involvement of both sides, or the council argues that clashes can occur between the own culture and potential dangers of minority cultures'.91 ${ }^{91}$ The conflicting two perspectives also involved an issue of internal hierarchy. Although the council formally decides upon the texts of reports, the staff members who regularly do most of the writing are also given an important voice. Despite a majority view in the council, the staff member who had fulfilled an important task in writing the drafts continued to resist a text that would stress the 'potential dangers of minority cultures'. In fact, he threatened to 
leave the project, believing that the emphasis on 'the protection of the own cultural achievements $[\ldots .$.$] too strongly suggests [. .$.$] that the Dutch major-$ ity is with its back against the wall [...] while in fact we have a dominant position'. 92

The chairman (of both the WRR Council and of this particular project group) played an important role in resolving the internal controversy. It was important for WRR to present its report unanimously to the outside world. Failure to do so would call its authority into question. As a result, the chairman took charge of re-editing the draft texts and attempted to develop compromises that would draw the involved actors back into the project. While references to possible cultural clashes were inserted into the draft texts, they were formulated conditionally. The new wording avoided assumptions regarding the danger of minority cultures, suggesting instead that if such dangers or conflicts were to emerge, the cultural achievements of Dutch society would have to be defended.

After several interruptions [...], the chairman argued that it would be best if he took over. He then devised formulas that would be agreeable to the two opponents, leading to several magical formulas. [For instance,] in those cases of confrontation where, in practice, no comprise is possible, no other option is available other than to defend the achievements of our culture against opposing claims. (xxii)

The passage above is very conditionally formulated; if this extreme case occurs, then [...]. So, the incompatibility of cultures was not formulated as the normal situation, but in a more conditional way. ${ }^{93}$

Despite the ferocity of this conflict and the role that hierarchy played in it, the clash constituted a crucial moment to allow for critical reflection regarding the frame for a minorities policy. The multiculturalist frame that had emerged in research and policy received criticism concerning its supposedly cultural relativist traits. This issue also sparked fierce controversy within WRR's back office. Risk of politicisation and taboos in public debate notwithstanding, WRR provided an ideal context for confronting this frame with alternatives. It provided a more pragmatic setting for adapting the problem framing as necessary. Within WRR, the multiculturalist frame that had emerged among specific research and policy actors in the preceding decade was critically reflected upon, adapted in terms of those elements that critics found too relativist and formulated into a policy frame for a minorities policy. This critical debate on the level of problem framing was to have an important effect on the policy changes to come. In fact, the government eventually adopted references made in the WRR report to cases where compromises would be impossible, choosing to reflect, in particular, the position of the individual in the policy. ${ }^{94}$ 


\subsection{Conclusion}

Research-policy dialogues clearly played a key role in the construction of the Dutch multicultural model of immigrant integration in the late 1970s and early 1980s. This was a formative period for both policy and research in this domain. For the first time, the government recognised the permanent status of immigrants, while researchers showed increasing interest in the social position of these minorities. A technocratic research-policy nexus allowed for significant co-evolution of developments in both fields during this formative period. In fact, the ethnic minorities policy of the 1980s was founded directly on reports and studies by WRR and ACOM. At the same time, the pronounced policy orientation of researchers also contributed to a dominant position of the so-called 'minorities paradigm' in the field of research. This co-evolution brought an end to the initial multiplicity of frames of immigrant integration and created a powerful institutional framework for a dominant multicultural frame.

The technocratic research-policy nexus was the product of strongly convergent patterns of boundary work in the fields of research and policymaking. Reflected was the then widely shared belief in rational societal steering, as manifest in many areas of social policy, and the broader tendency of social scientists to be policy-oriented. An important factor in this domain was that WRR was an institutional given, providing science-based advice to policymaking.

However, there are also important factors that account for the construction of a technocratic nexus in this specific domain. On one hand, policymakers not only believed that this problem could be resolved in a rational manner within a decade or so with the help of social scientists, but they also strained to depoliticise the topic. This was manifest in CRM's missionary boundary work, which mobilised research in an effort to raise support for the development of a minorities policy, and the positivist boundary work of the Minorities Policy Directorate, which endeavoured to define immigrant integration as a non-political issue that had to be developed on the basis of rational insights from independent research. On the other hand, researchers involved in this nascent field felt a strong sense of societal engagement with the position of minorities, something influenced by the dominant anthropological tradition in this field. This clearly applied to ACOM, which stressed the societal function of research, as well as to WRR, which worked hard to prove its societal and political function in this period. However, the technocratic nexus implied that other sorts of expertise, i.e. 'ethnic expertise', would become less influential. This is a marked contrast with other areas of social policy, where tripartite organisations often played an important role involving researchers and policymakers, as well as group representatives. 
The technocratic nexus contributed to the rise of the multicultural model in a very particular way. It created a structural symbiosis between specific actors who were advocating a multiculturalist, rather than differentialist, frame. In this way, it generated positive feedback regarding frameshifts in research and policy. It did this by strengthening the position of certain actors involved in this symbiosis (ACOM, WRR, CRM, Minorities Directorate) and weakening that of others (Department of Social Affairs, welfare organisations). Furthermore, the symbiotic relationship between a relatively small network of actors enabled combined focus on what these actors considered to be specific to minorities - their ethnic and cultural status. This created what can be described as the 'logic of minorities' in problem framing, contributing specifically to the rise of a multiculturalist frame.

However, the technocratic research-policy nexus does not seem to have been designed to promote a critical dialogue between research and policy at the level of problem framing. It offered some opportunities for frame reflection, in part by breaking open the iron triangles that had formed around the differentialist approach of the 1970s. At the same time, however, it limited the debate to a select set of actors, excluded possible alternative frames, took the multiculturalist frame as a starting point rather than as an object of reflection, limited pragmatism because of the sensitivities of the issue and limited trust to a select group of actors. The nexus, then, was a product of strategies designed to reinforce the structural positions of actors with a multiculturalist problem frame. Thus, the research-policy nexus in this period clearly enjoyed an important structural meaning in research and policy frameshifts, though not in a way involving critical frame reflection. The rise of the multicultural model was the outcome of strongly convergent forces in research and policy - forces that supported depoliticisation and the logic of minorities in problem framing, rather than opening reflection on various frames. 



\section{Enlightenment and the rise of universalism (1989-1994)}

The multiculturalist approach that had been developed in the early 1980s remained the cornerstone of formal Dutch policies throughout the decade. This is the period for which the Dutch approach would become so internationally known as a representative of the multicultural model, although Dutch policies were rarely phrased in terms of 'multiculturalism'. In the 1980s, the ethnic minorities policy was institutionalised and many of its elements implemented. Even though many areas of social policy in the Netherlands were reformed during this decade due to economic depression and a stringent politics of welfare state retrenchment, for a long time the domain was relatively unaffected. In addition, immigrant integration research thrived during this period, with ACOM maintaining its central position on the research-policy nexus and an ever larger network of researchers becoming involved in this field. Immigrant integration research had evolved into a vast industry.

However, a shift in formal policy discourse did take place in the early 1990 s, with the development of the integration policy, which had distinct universalist traits. Moreover, the dominance of the minorities paradigm became challenged by the end of the 1980s. In this chapter, we will see that pressure for policy change started to build during the late 1980s, though a formal policy change took place only years later. In the early 1990s, the famous Dutch multicultural model was replaced in official policy discourse by a more universalist approach that phrased immigrant integration in terms of 'integration' and 'citizenship' rather than emancipation. It labelled immigrants as a single category of individuals ('allochthonous') rather than as ethnic or cultural groups ('minorities'). Furthermore, it chose to explain immigrant integration in terms of the social-economic participation of migrants instead of the social-cultural emancipation of minorities. In sum, a liberal-egalitarian perspective came to override any normative focus on multiculturalism.

This frameshift challenged the established research-policy nexus. However, it also presents a very interesting case for how research and advisory bodies can provide a venue for putting new ideas on the agenda. In the depoliticised setting of the 1980s, it was a second report from WRR that would provide an alternative to the multicultural model on the agenda. The main focus in this chapter will be to analyse the configuration of 
research-policy relations in this period and reconstruct the role of researchpolicy dialogues in the turn away from the traditional Dutch multicultural model.

\subsection{The turn towards universalism}

The first step is to picture the players, the playing field and the societal context in which the frameshift from multiculturalism to universalism occurred. It will become evident that new actors with other frames were gradually emerging, beyond the structural symbiosis that had supported the multicultural model in the 1980s.

\subsubsection{Context: Ongoing immigration and welfare state retrenchment}

The frameshift towards universalism involved a break from the 1980s' relative stability in immigrant integration policies. This equilibrium was sustained by the structural symbiosis between research and policy, as analysed in the previous chapter, which effectively established a structure-induced subsystem that was relatively insulated from external developments (Timmermans \& Scholten 2006).

However, various contextual developments eventually contributed to a disturbance of this structure-induced equilibrium. First, an economic recession during the 1980 s led to a surge of unemployment in the society at large and among immigrants in particular. Migrants were especially vulnerable to economic decline and were hit hard by the reform of the labour intensive sectors in which they were often employed. Whereas unemployment had been relatively low in the 1970s, especially among foreign workers, it skyrocketed in the early 1980s. In 1984, the level of unemployment among Moroccans and Turks was about double the unemployment among native Dutch (35.5 per cent and 38.8 per cent, respectively) (Koolen \& Tempelman 2003: 53). In 1987, the differences in unemployment levels had become starker: 13 per cent for natives, 27 per cent for Surinamese and no less than 42 per cent and 44 per cent for Moroccans and Turks, respectively. ${ }^{1}$ In addition, the position of migrants in the educational sphere appeared to lag behind that of the population at large. This included a relatively low level of education, low educational participation and high levels of school dropout (WRR 1989: 139-141).

From the 1980s onwards, successive governments conducted a politics of retrenchment in various policy areas. Retrenchment politics was deemed necessary to maintain the viability of the welfare state. This involved a socalled 'no-nonsense politics' aimed at cutting back welfare spending by changing welfare state facilities (cutting back on social benefits, restricting access to social benefits) and prioritising employment (putting work before 
income). A 'regulated liberalisation' of the welfare state took place at this time, involving a recalibration of the division of responsibilities between state, civil society and individuals (Van der Veen \& Trommel 1999). Gradually, welfare responsibilities shifted from the state towards the individual or the market. The level of unemployment decreased during the 1980s, but stagnated towards the end of the decade at just below 14 per cent. It remained especially high for immigrants in the second half of the 1980s (Koolen \& Tempelman 2003: 73).

The ongoing influx of migrants during the 1980s - and particularly, continued family migration in the form of family reunion and family formation - frustrated the expectation that immigration could be effectively brought to a halt while integrating those minorities that had settled in the Netherlands. Furthermore, asylum migration in the second half of the 1980s shot up (VWJ 2004: 244). In previous decades, asylum migration had taken place on a relatively small scale and mainly within Europe. Now, the waves of asylees included various 'new' groups from Sri Lanka (Tamils), Ghana, Somalia, Iraq, Iran, Ethiopia and Afghanistan. As a result of both family and asylum migration, a positive migration balance in the Netherlands remained throughout the decade - from a positive migration balance of 53,000 in 1980 , numbers decreased to 24,000 in 1985 only to rise again to 60,000 in $1990 .^{2}$ Moreover, the number of immigrants or 'allochthonous' - defined as persons either born outside of the Netherlands or who have at least one parent not born in the Netherlands - rose from 1.3 million (9.9 per cent of the total population) to 2.1 million (14.2 per cent of total population) between 1975 and the 1990s. ${ }^{3}$ This meant minority groups that had been formally recognised as policy targets grew significantly larger: figures for 1990 show 237,000 Surinamese, 206,000 Turks and 168,000 Moroccans living in the Netherlands (Martinez \& Groeneveld in VWJ 2004). These groups also became increasingly diverse in terms of differentiation between first- and second-generation migrants. It is also important to note that asylum migration led to the arrival of 'new' immigrants who were not recognised as 'minorities' in the minorities policy and who received little attention from researchers.

In response to these developments and particularly to the signs of stagnation in the social-economic position of minorities, the government expressed doubts about whether the current approach was sustainable. An advisory request was issued to WRR for a new report on immigrant integration, which was published in 1989 (WRR 1989). This report is generally seen as a turning point in both policy developments (Blok 2004a; Entzinger 2006; Penninx 2005) and research developments during this period (Engbersen \& Gabriëls 1995b; Timmermans \& Scholten 2006). WRR advised the government to develop an integration policy that would focus more on the social-economic activation of individual migrants. 
As the reply memorandum to this report indicates, the government did not immediately adopt this new perspective. ${ }^{4}$ However, it did mark a shift in public and political discourse on immigrant integration (Fermin 1997: 82). This shift in discourse was contested by some of the actors that were involved in the structure-induced equilibrium of the multiculturalist approach. Molleman, director of the Minorities Policy Directorate until 1990, reported to the media that he had 'doubts whether a fundamentally new policy approach was needed'. He claimed that although the minorities policy may not have been entirely successful, it could also not be dismissed as a failure: 'Policy has not failed; it has only not yet succeeded'.5 Molleman also objected to plans for budget cuts to the minorities policy. He claimed that, especially in bad economic times, efforts made to achieve the integration of these groups should not be diminished. ${ }^{6}$ In addition, Penninx, a civil servant at CRM and an ACOM observer, wrote a number of journal and newspaper articles in which he referred to the criticism of the multiculturalist policy approach as 'democratic impatience'. He made the point that the effects of this approach would take considerable time to pay off (Vermeulen \& Penninx 1994). ${ }^{7}$ ACOM also rejected claims that a different policy approach was necessary. This is illustrated by a response it published in the 1989 WRR report whereby it discarded the claim that the policy had failed (ACOM 1989). Migrant organisations also criticised the new mode of discourse, sometimes using terms such as 'forced integration' or 'hidden assimilation' (Blok 2004a: 116-117).

However, the minorities policy gradually shifted from a specific approach on minorities to an intensification of general policies and, in particular, (mainly social-economic) policy domains such as labour and education (Fermin 1997: 82). Reflecting a more general trend towards decentralisation, municipalities came to have greater power. Due to this functional and territorial decentralisation, the coordinating task of the Department of Home Affairs grew weak (Molleman 2003). One of its most important means for policy coordination was orchestrating research and monitoring policy efforts and effects in various areas. In this respect, it cooperated closely with the SCP, which would become an important provider of regular minorities reports containing data on the social-economic status of migrants.

In the early $1990 \mathrm{~s}$, immigrant integration became an increasingly central issue in both public and political debate. In 1990, Prime Minister Lubbers openly expressed his disappointment with the effects of the minorities policy and called for a more activating approach to immigrant integration. ${ }^{8}$ Furthermore, following political statements by leader of the main opposition party Bolkestein, a broad national minorities debate emerged in 1992. This debate played out in the media (Mariën 1992), in politics (Koolen 2003: 27) and in several conferences organised by the Minister of Home Affairs. ${ }^{9}$ It drew attention to social-cultural issues of immigration and to 
concerns about social cohesion. In response to this debate, experts Entzinger and Van der Zwan wrote an advisory report to government that elaborated plans for civic integration programmes for newcomers to Dutch society. These were the same plans that had already been raised in the 1989 WRR report (Van der Zwan \& Entzinger 1992). Civic integration programmes would become an important facet of policy in the 1990s and their focus on all migrants instead of specific minorities, as well as on the social-economic proficiencies of migrants rather than their social-cultural status, clearly indicated a shift towards universalism.

In response to the gradual changes that had taken place in the preceding period, Parliament called for a 'recalibration' of the contours of government policy to replace the minorities memorandum. To achieve this, a contours memorandum on the integration of ethnic minorities was presented by the Department of Home Affairs in 1994. As observed in chapter 4, this memorandum marked the shift from the multiculturalist (with universalist traits) minorities policy towards a more universalist integration policy. As for research, this period also marked the evolution of a more universalist 'integration paradigm' alongside the multiculturalist 'minorities paradigm'. WRR and SCP seem to have been especially pivotal in this respect. ACOM, however, was discontinued in 1992.

To recap, various research and policy actors contributed to this frameshift. In research, this included WRR, which published a second report on immigrant integration, SCP as a data provider, experts Van der Zwan and Entzinger who published a report in response to the national minorities debate as well as ACOM, which appears to have lost its central position in this period. In policy, this included the Department of Home Affairs, which remained the coordinating body even though its role changed during this period, as well as political actors who became more involved in policymaking at the time.

\subsubsection{Established nexus: The Department of Home Affairs and ACOM}

The shift towards a more universalist frame of integration took place in a setting already featuring an institutionalised research-policy nexus. The technocratic symbiosis of the 1980 s formed an important part of the decade's strongly centralised and unitary structure for policy coordination (Guiraudon 2000b: 131-134), which was reinforced by the co-optation of ethnic elites. While the responsibility of welfare organisations was increasingly marginalised, the role of immigrant self-organisations had become more structural, especially when the National Advisory and Consultation Structure for Minorities (Landelijk Advies en Overleg Orgaan voor Minderheden, LAO) was established in 1984. In order to appreciate the setting of actors in research-policy relations, it is first necessary to sketch the positions and frames that were developed at the time. 


\section{The Department of Home Affairs}

The Department of Home Affairs - more precisely, the Minorities Policy Directorate within it - was responsible for the interdepartmental coordination of minorities policy during the 1980s. Headed by Molleman, it initially held a distinct coordinating position and often undertook new policy initiatives, even when they were to be implemented by other policy departments. However, this task grew problematic the 1980s, as various policy departments claimed more and more autonomy. ${ }^{10}$ In fact, during the second half of the decade, the department became subject to growing political pressure (Koolen 2003: 25), as a result gradually shifting from a stance of strong to weak policy coordination (Molleman 2003). ${ }^{11}$ This meant that instead of initiating policy programmes across areas, it was now constantly searching for new ways to convince other departments to accept specific policy measures. This change in its structural placement was also illustrated by renaming the directorate the Directory for the Countours of the Minorities Policy. ${ }^{12}$ In the contours memorandum of 1994, policy coordination was defined as the 'shared responsibility' of national and local governments, and it was stressed 'that the centre of gravity in the realisation and implementation of policy lies mainly with the municipalities'. ${ }^{13}$

The weaker coordinating role held by the Minorities Policy Directorate reflected a broader structural trend of territorial and functional decentralisation. At this time, the government conducted a politics of retrenchment in various areas, including immigrant integration and welfare state policies. Also clearly affected was the centralised and unitary structure of the minorities policy, which was increasingly delegated to sector departments, as well as to local governments. The department's loss of power was one reason Molleman resigned as director of the Minorities Policy Directorate in 1990 (just after publication of the reply memorandum to the WRR report from that year). ${ }^{14}$

In addition to change in the department's leadership, a different problem framing was also adopted in this period. It shifted from a multiculturalistwith-universalist-traits frame to a more universalist one, as demonstrated by two documents: the reply memorandum to the 1989 WRR report on 'Allochthonous policy' (1990) ${ }^{15}$ and, to a greater degree, a new memorandum developed by this department on the coordination of the integration policy, the contours memorandum on the integration of ethnic minorities (1994).

The reply memorandum was written by the Interdepartmental Committee for the Minorities Policy, chaired by the Department of Home Affairs, on behalf of the government coalition that was established in 1990. Not only did it signal this department's status at the time, but also the changing political views on immigrant integration that were attached to it. The reply memorandum maintained the original policy contours set by the 1983 minorities memorandum (Fermin 1997: 194). It contained, for 
instance, the social classification of immigrants as 'minorities' and focused specifically on a number of selected minorities, with the understanding that 'Dutch government carried a special responsibility for the admission of these groups or groups that were connected with the Netherlands through the colonial past'. ${ }^{16}$ Furthermore, it argued that the minorities concept was 'internationally accepted and referring to fundamental principles of democratic law, especially concerning the principle of respecting minorities' (ibid.). It also stated that measures aimed at the institutionalisation of cultural differences, such as language classes for immigrants and the Advisory and Consultation Council for Minorities, were to be maintained.

However, the memorandum also showed signs of a minor shift towards a more universalist approach. It reiterated the demand for, as the slogan went: 'General policy wherever possible, specific or categorical policy where necessary'. ${ }^{17}$ In this respect, it proposed measures for functional and territorial decentralisation, including in the context of the politics of social renewal in this period. It concentrated more on social-economic concerns such as education and labour.

Integration does not conflict with identity; it adds a dimension to it, [...] only those who speak the Dutch language sufficiently, who have completed an adequate occupational education and who have learned to stand firm in a hard and competitive society, can effectively participate while retaining one's own identity. ${ }^{18}$

The government adopted WRR's recommendation to develop additional facilities for newcomers to acquire the necessary educational basis for participation in the labour market and in Dutch society at large. ${ }^{19}$ Furthermore, it embraced WRR's suggestions for an Equal Treatment and Equal Employment Act and also the recommendation to create a legal basis for dual nationality.

The fact that the Minorities Policy Directorate wrote the contours memorandum $^{20}$ provides a direct indication of the changing problem frame within the Department of Home Affairs. ${ }^{21}$ In the memorandum, the name of the minorities policy was changed to the integration policy. In terms of the concept of integration, the memorandum suggested that it would be 'better that the social integration of minority groups, and persons belonging to these groups is a mutual process of acceptation'. ${ }^{22}$ It also adopted a more liberal-egalitarian perspective on immigrant integration by stressing the individual obligations of migrants for their integration, stating that:

on all members of ethnic minorities that stay permanently in the Netherlands [...] lies the individual obligation to participate in education and the labour market and also the obligation to make efforts 
to learn the Dutch language and to acquire basic knowledge of Dutch society. $^{23}$

More so than the reply memorandum of 1990 , the contours memorandum diminished forms of institutionalised pluralism, for instance, by depriving the LAO of its advisory function. This arose out of fear that such a function would interfere too much with its consultative task and that it would result in a form of relative privilege for ethnic minorities over other groups in society (Blok 2004a:494).

\section{Advisory Committee on Minorities Research (ACOM)}

ACOM's central position in the established research-policy nexus solidified in the early 1980s and lasted throughout the decade. Its formal mandate was broadened from advising CRM to providing advice on minorities research to the government as a whole. During this era, it was formally associated with the Department of Home Affairs (Van Putten 1990: 362). However, ACOM's existence was subject to an establishment regulation for a set period. This timeframe had to be regularly renewed (standardly once every four years) in order for ACOM to continue. In 1984, the latest extension led to debates about whether to discontinue ACOM, in the context of a larger restructuring of government advisory bodies (ibid.: 363). The Minorities Policy Directorate strongly opposed a discontinuation, illustrating the strength of the established research-policy nexus in this period. $^{24}$

During the early part of the decade, ACOM's influence on research programming was very significant. Penninx, then working at CRM and acting as an observer to ACOM, even concluded that almost all of the projects suggested in ACOM's 1979 'Minorities research advice' had been adopted by the government several years later (Penninx 1988b: 35). Furthermore, ACOM would also have significant indirect effects on this research field at large, 'because of the infrastructural work of ACOM and the coordinating role of its secretariat' (ibid.: 37). Without doubt, ACOM had enormous influence on the development of the immigrant integration research field.

ACOM also maintained a multiculturalist problem framing during the 1980s. This meant that its advice often contained a specific focus on minority groups, with particular attention to issues such as the social-economic status of migrants; deprivation (due to, for example, discrimination); participation, emancipation and culture; problem accumulation; and women, youth and the elderly (ibid.: 32). These were also the topics of government research programming, which ACOM regularly advised be consolidated. ACOM's influence on government research programming included drawing attention to issues of discrimination, as manifested in key advice from ACOM in the $1980 \mathrm{~s}$. It took the form of 'A fair chance', a report by 
Bovenkerk (1987) on positive labour market measures for stimulating migrant participation by forcing obligatory measures on employers.

ACOM's lead changed during the second half of the 1980s. Penninx (1992) and Van Putten (1990) relate the decline of this body to the institutionalisation of the minorities policy in that period, which diminished the demand for the type of expertise ACOM could provide and upped demand for more evaluative research. Penninx (1988b: 36) observes how although some ACOM reports on policy developments were very influential, others remained largely ignored. In 1987, significant changes took place (Van Putten 1990: 365): Köbben resigned as ACOM chair to be replaced by Bovenkerk, and Van Amersfoort left the committee. At the same time, various new researchers joined, including several from minority groups.

In 1992, the Department of Home Affairs chose not to extend the mandate of ACOM and the body was discontinued. It was succeeded by a Temporary Scientific Committee for the Minorities Policy (Tijdelijke Wetenschappelijke Commissie Minderhedenbeleid, TWCM), though TWCM differed significantly from ACOM in a number of respects. Firstly, it was not, like ACOM was, a technical-scientific committee; instead, it was bipartite, consisting of researchers and policymakers. Secondly, it was primarily intended to further the translation of existing research and expertise for the purposes of policy practice. TWCM was dissolved in 1996.

\section{Migrant self-organisations}

Last but not least, the centralised and unitary structure in this issue domain during the 1980s also involved migrant self-organisations. The importance of welfare organisations, or so-called zaakwaarnemers, diminished in the early part of the decade to be replaced by a new structure of migrant selforganisations. Formed in 1984, the LAO served in an advisory capacity concerning policy formulation at the Department of Home Affairs and held a consulting role in obtaining insights for government from and about minorities. These organisations were also involved in providing advice on research programming, which had become fiercely contested by the end of the 1980s. However, their influence on research programming seems to have been rather limited. ${ }^{25}$

These organisations maintained the multiculturalist frame that formed the basis for the policy and research structure in this period. For instance, in a joint response with the LAO to the government's reply memorandum to the 1989 WRR report, the migrant self-organisations advocated a consolidation of the broader approach to social-economic deprivation, collective emancipation and identity, rather than adopting a narrower approach to social-economic participation (Fermin 1997: 195). Furthermore, these organisations focused attention on issues such as discrimination and structural deprivation rather than a universalist focus on individual deficiencies of migrants (ibid.: 196). They also continued to frame immigrant integration in 
the context of a normative process of transforming Dutch society into a multicultural society, rather than adopting a more liberal-egalitarian view on immigrant integration (ibid.: 199).

These actors from the prevailing research-policy nexus interpreted the evidence about ongoing problem developments in a way that was different from other actors. They selected evidence about the multiculturalist approach's success rather than its failures. Much, for instance, was made of the legal and political position of minorities, which had significantly improved during the $1980 \mathrm{~s}$, as well as the implementation of a constitutional right to vote in local and regional elections for long-term resident foreigners. Furthermore, researchers and policymakers both often defended the Dutch multiculturalist approach internationally, proclaiming that the absence of any significant racial unrest in the Netherlands during this era was a sign of its success, in contrast to what several surrounding countries were experiencing (Vermeulen \& Penninx 2000).

At the same time, the persistent deprivation of migrants in socialeconomic domains such as labour and education was interpreted not as an indication that the current approach had failed but rather, as confirmation of the need to continue currents in the same direction. It was argued that in periods of overall economic decline, a specific approach to minorities was even more necessary so as to avert the disproportionate effects that such trends could have on the status of minorities. For instance, the director of the Minorities Policy Directorate frequently stated that the integration policy had not failed, but it had not yet succeeded. Contrary claims were sometimes rejected as issues of 'impatience'. This clearly reflected the 'negative feedback' mobilised by the established research-policy nexus from the 1980 s in response to mounting pressure for policy change.

\subsubsection{Scientific Council for Government Policy (WRR): Ethnic minorities II?}

A decade after its first report on immigrant integration entitled 'Ethnic minorities' (WRR 1979), WRR published a second report on this issue, 'Immigrant policy' (WRR 1989). During the 1980s, WRR had continued the more in-depth approach to specific policy themes that it had developed in the period of its 1979 report. Furthermore, WRR had become increasingly institutionalised during the 1980s, gaining significant authority in the field of both policymaking and research. This authority was reinforced by the appointment of former Minister of Social Affairs Wil Albeda as WRR chairman in 1985. The 1979 report had a direct influence on the authority gained by WRR, specifically in the fields of immigrant integration research and policy. It is important to note here the statutory requirement for the rotation of council members every five years. Whereas one of the main authors of the 1979 report was a WRR staff member who went on to be involved in the development of the second report, there was no direct 
connection between these two reports in terms of council members - the main author of the second report, Entzinger, had not been involved in the 1979 report, although he was ACOM secretary at that time.

Another important difference is that the second report was a response to a formal advisory request from the government. A 1988 government document entitled 'Action programme minorities policy' concluded that insufficient improvements were being made in crucial areas. ${ }^{26}$ Reference was made, in particular, to indications of rising unemployment and persistent social-economic deprivation among migrants. It was against this background that the government requested new advice from WRR on immigrant integration. The advisory request's text stated that on 'material points', such as housing, education and labour, 'too little progress' had been made; whereas 'on a number of, mainly immaterial, points significant progress has been made'. ${ }^{27}$ The document raised doubts about 'whether the approach that characterised the policy from the minorities memorandum [...] would have to be continued in its current form'. WRR was asked to provide 'advice for the prioritisation of future government policy' so that 'strategic choices on the future of the minorities policy could be made in 1990 [...]'. Furthermore, it was asked to provide 'creative and practical suggestions' on how to ameliorate the policy design. A remarkable move for its relationship with WRR at the time, the government set a deadline to receive this advice before making strategic choices in 1990.

However, this report provided more than just an update of the previous report in the context of ongoing issue developments. It contained a very different perspective in various respects. It renamed immigrant integration in terms of 'integration' instead of 'emancipation', defining integration as 'equal participation in societal sectors and institutions'. ${ }^{28}$ It framed immigration as a permanent phenomenon rather than a historically unique occurrence, based on the 'supposition that the level of migration to the Netherlands [...] will be sustained for the foreseeable future'. ${ }^{29}$ It stated that 'whereas the council advised in 1979 that the presence of immigrants in Dutch society should be regarded as a permanent phenomenon, it now expects that immigration too will be a lasting feature'. ${ }^{30}$

Rather than being classified as ethnic or cultural groups, migrants were now defined as a social category. The report also proposed use of the - difficult to translate - concepts of 'allochthonous' (allochtonen) and 'autochthonous' (autochtonen), referring to 'all those who have migrated to the Netherlands plus their descendants up to the third generation, insofar as the latter wish to regard themselves as non-indigenous'. 31 Based on this more open definition of immigrants, the policy called for 'periodical review' to determine which immigrant groups were in a low social position and would therefore be eligible as a target group for government policy. However, critics rejected classifying and selecting specific minorites as policy target groups, saying it was 'arbitrary and prompted more by 
historical than by social considerations'. ${ }^{32}$ Furthermore, because the minorities concept referred only to those groups of a different ethnic or cultural origin who occupied low social status, it would be 'too limited a concept' for describing the social 'dynamism' found among immigrants and would be 'stigmatising'. 33

This reclassifying of migrants reflected the distinct causal theory elaborated in this report. Instead of approaching migrants as separate groups, WRR considered the position of migrants 'in significant measure the product of general developments'. Therefore, 'any policy specifically directed towards minorities should be primarily conceived as an intensification of general policies in the sectors in question'. ${ }^{34}$ Integration would have to be achieved by general policy measures rather than by group-specific measures. Furthermore, such measures should be aimed at enabling the migrant 'to stand on his own feet', or to promote citizenship of migrants on the whole. The role of government policy would be a facilitating one, as migrants not only have 'rights' to entitlements but also 'obligations' to participate in society. 'All the state can do is to help ensure that the right conditions exist [...] on the part of immigrants, a commitment is required to make full use of the facilities on offer'. ${ }^{35}$ In this respect, WRR also envisaged problems for migrants carrying dual nationality, especially should it encourage the naturalisation of migrants in Dutch society. ${ }^{36}$

WRR developed a universalist rights-and-duties perspective on immigrant integration. On one hand, government should provide conditions for migrants to be able to participate in society. WRR proposed the development of an Equal Treatment Act for combating discrimination and an Equal Employment Act to exert soft pressure on employers to hire migrants. On the other hand, WRR also pushed the obligation on migrants to participate so as to remain eligible for government facilities in various spheres. The belief was that the state should be able to impose 'penalties where those opportunities are not exploited'. 37 Whereas multiculturalism focuses on processes of position attribution by processes in society at large, such as deprivation due to discrimination, this more universalist perspective emphasises position acquisition by migrants themselves, such as citizenship and participation. In fact, WRR rejected an approach that would treat migrants as 'welfare categories' who are dependent on government facilities.

[...] [G]overnment tends to view these groups too much in the light of welfare categories instead of providing them with opportunities to stand on their own feet. Many members of minority groups have become directly or indirectly dependent on the state in the form of social security benefits, welfare services and facilities and housing. ${ }^{38}$ 
Finally, WRR adopted a different perspective on the multi-ethnic, multicultural character of Dutch society. Rather than seeing multi-ethnic society as a normative ideal, it accepted it as a 'social datum' in which government should not intervene. Institutional cultural pluralism was rejected. Instead, culture and ethnicity were attributed to the private sphere beyond the reach of government policy. In fact, one of the reasons the minorities policy was so minimally effective is that it remained 'stuck in a debate on policy goals ${ }^{39}$ with a 'symbolic' character, focusing especially on 'cultural and morality' instead of on vital problem areas. ${ }^{40}$

[...] [T]he institutionalisation of ethnic pluralism need not be regarded as an independent objective of government policy. A multiethnic society should be regarded as a social datum and, hence, as a starting point for policies leaving space for cultural diversity in various fields. [...] Immigrants who so wish should be able to maintain and develop their own cultural identity: integration certainly does not imply cultural assimilation. To an even greater extent than institutional integration, however, this forms part of the responsibility of the individual groups. [...] The government's task is confined to helping eliminate the barriers experienced by ethnic groupings as a result of their non-indigenous origins, with a view to enabling them to participate on a equal footing with indigenous people in a culturally diverse society. ${ }^{41}$

\subsubsection{Social and Cultural Planning Office (SCP)}

In the early 1990s, another actor obtained a more central position in the field of immigrant integration research: SCP. Since this period, the office has published annual, and later biannual, minority reports containing mainly quantitative data on the social-economic status of migrants.

SCP has an institutional history similar to that of WRR. Both were established in response to recommendations by the De Wolff Committee. SCP was to counterbalance the economic expertise that was provided by the more established Central Planning Bureau (CPB) (Halffman \& Hoppe 2006). It was formally part of CRM, which takes responsibility for coordinating social-cultural policies. However, like WRR, it enjoys formal independence in its work programme. SCP evolved out of the Research and Planning Directorate of CRM from the 1960s and early 1970s. Despite the strong orientation of this directorate towards ethnic minorities, SCP attributed little systematic attention to immigrant integration in the second half of the 1970s and the early 1980s. For instance, only the 1976 and 1986 editions of its biannual social and cultural reports contained chapters on ethnic minorities (Van Praag 1986: 2). 
SCP did get more involved in immigrant integration from the second half of the 1990s. At the request of the Department of Home Affairs in 1984, it published a report on immigrants' use of social facilities - for instance, welfare state entitlements (i.e. social security) - in the spheres of education, labour and housing (Van Praag 1984). This showed that SCP was primarily focused on the participation of migrants in social-economic domains. Furthermore, SCP paid significant attention to ethnic minorities in its 'Social and cultural report' from 1986. This report was very critical of the claim that the Netherlands would become a multicultural society. It claimed instead that there was no evidence of the development of ethnocultural 'streams' in Dutch society, which placed primary importance on minorities' ethnic or cultural status (Van Praag 1986: 44). Moreover, Van Praag, who authored a chapter on ethnic minorities in SCP's 1986 report, observed in another article that he did not perceive Dutch society as multicultural and believed that the phrase 'mutual adaptation between minorities and majority' written in the 1983 minorities memorandum placed too much emphasis on the adaptation required from the side of the majority society (ibid.: 45). Since 1990, SCP has provided the government regular minorities reports containing an evaluation of the status of migrants, mainly in social-economic domains such as education, labour and housing. Publication of these reports allowed SCP to take on a more influential position in the immigrant integration research field.

SCP's involvement in this period appears to reflect a universalist problem framing, as illustrated through its focus on, in particular, the socialeconomic participation of migrants and its explicit rejection of multiculturalism. Furthermore, this universalist problem framing was also manifest in its methods of data collection that did not involve a differentiation for specific groups. In fact, until well into the 1990s, SCP studies were based on generic data that had been derived from various sources within the National Statistics Office (Centraal Bureau voor de Statistiek, CBS), such as the Labour Force Survey (Enquête Beroepsbevolking, EBB).

\subsubsection{The Van der Zwan and Entzinger report (1994)}

Another instance where research actors were involved in research-policy relations in this period was the advisory report published by experts Entzinger and Van der Zwan. Both had previously helped produce WRR reports on immigrant integration - Entzinger as one of the authors of the 1989 WRR report and Van der Zwan as a council member who was pivotal in the development of the 1979 WRR report.

Following the national minorities debate of 1992, Van der Zwan was initially asked by the Minister of Home Affairs to be involved in organising a series of conferences. In the context of these conferences, Van der Zwan wrote a memorandum to the Minister of Home Affairs (28 September 
1992) that contained 'a fairly complete compilation of all possible data on the position of minorities, including negative aspects, criminality rates and reliance on social facilities' ${ }^{42}$ This report was debated during a special parliamentary session of the national minorities debate. ${ }^{43}$ However, its impact on concrete policy measures was limited. ${ }^{44}$

Van der Zwan and Entzinger felt that, although the national minorities debate had created a political sense of urgency, it tended to focus too much on cultural aspects of integration (as raised by then leader of the Liberal Party Bolkestein), and that it had led to insufficient concrete policy measures. ${ }^{45}$ When the 1992 memorandum by Van der Zwan also failed to elicit significant policy consequences, Van der Zwan and Entzinger lobbied the Department of Home Affairs for more appropriate policy measures. This led to the two men receiving a formal assignment from the department to write a new document. It would come to be called the 'Policy succession of the minorities debate', having a significant influence on the development of civic integration programmes, which would become a central element of the 1990s integration policy (Blok 2004a: 116).

Many facets of the 1989 WRR report were revived in this advisory report, including its universalist way of framing immigrant integration. It named and framed immigrant integration in terms of integration, civic or otherwise, among newcomers as new citizens. The rights-and-duties perspective and the focus on integration policy as intensified general policy also took further shape in this report. For instance, it argued that 'integration is promoted in the first place through labour market participation' (Van der Zwan \& Entzinger 1994: 1). To this end, the report recommends temporarily lowering the minimum wage for newcomers, in order to facilitate their inclusion into the labour market. This temporary limitation of immigrant rights had to be combined with an obligation to provide civic integration programmes, directed at language and civic skills. Immigrants were granted access to civic integration programmes that would allow them to become full members of Dutch society but, in turn, they were obligated to participate.

From a normative perspective, immigrant integration was framed in the context of maintaining a viable welfare state. Added to this was a liberalegalitarian concern about social cohesion. Van der Zwan and Entzinger wrote, 'from the perspective of social cohesion, as well as the stability of our welfare state, we are facing a fundamental turning point' (ibid.: 2). Immigrants' disproportionate reliance on welfare facilities would threaten the solidarity required for maintaining a viable welfare state. The report argued that:

when policy remains unaltered, a mismatch will develop [...] between supply and demand on the bottom of the labour market, which can lead to tensions between the established population and 
parts of newcomers and will lead to an increase in demand for facilities of the welfare state, especially by immigrants. (ibid.: 14)

This was put in the perspective of both a broader development in welfare state orientation and in the rising concerns about cultural relativism after the second national minorities debate.

The urgency of a solution for this issue [...] is further underlined by the societal shift, also on a European level [...], in the character of the welfare state, from 'soft compensatory' in the direction of 'achievement performance'. [...] The willingness of society to compensate for differences declines and the pressure to make a productive contribution increases. Minorities that do not take part in this societal reorientation will increasingly acquire an isolated position, with all the accompanying social tensions. (ibid.: 5)

In 1994, the plans for civic integration programmes were adopted in a coalition agreement of the new purple government that was formed in that year. However, neither the observations about a more obligatory approach, nor plans to allow selective lowering of minimum wage levels were taken up in the coalition agreement. Plans for civic integration programmes also appeared in the contours memorandum integration policy that was published just before the Van der Zwan and Entzinger report. While the memorandum did refer to the 1989 WRR report that had already proposed such programmes, it did not adopt the obligatory approach.

\subsubsection{Politics}

Finally, political actors became more involved in policymaking and research-policy relations in this period. Until the 1980s, politicians had played a relatively minor role in policy developments. As we saw in the preceding chapter, this was due, in part, to the inarticulate positions of political parties in this area. There was also a tendency to depoliticise this issue and to adopt a positivist approach to policymaking that, for the most part, involved researchers and administrators and perceived politics as a threat to a rational and consistent approach.

Then Prime Minister Lubbers, of the Christian Democratic Party (CDA), was one of the first to break with the tendency to depoliticise immigrant integration. As Prime Minister of various successive governments between 1982 and 1994, Lubbers had a significant hand in the politics of welfare state retrenchment, which had already affected many policy domains but, as previously mentioned, not yet immigrant integration. In a radio interview that became widely discussed in the media and Parliament, he drew attention to the relatively high degree of dependence of migrants on 
welfare state facilities and called for 'less soft treatment'. ${ }^{46}$ He claimed he 'was losing his patience' with minorities and that he wanted a policy revision that followed the lines of the 1989 WRR report. ${ }^{47} \mathrm{He}$ further argued that the minorities policy should primarily involve an intensification of government policies in various (social-economic) sectors and should therefore also fall under the remit of those departments responsible for these specific sectors. In this respect - and also in line with the 1989 WRR report - he claimed that a directorate for the coordination of the minorities policy would be redundant, as responsibility for migrant policy would shift towards various sector departments. ${ }^{48}$

To some extent, his statements reflected the rise of a more economicliberal perspective in the party ideology of the CDA (Fermin 1997: 125), which stressed the responsibilities of citizens, including migrants, to participate in Dutch society. This welfare state perspective on immigrant integration complemented, rather than substituted, the CDA's stance on achieving immigrant integration by means of collective emancipation (ibid.: 130). In fact, in this period Lubbers was still defending 'pillarism', a form of institutional multiculturalism or even differentialism, as a model of immigrant integration. ${ }^{49}$

Another political entrepreneur who had an important effect on policymaking was the leader of the main opposition party in this period, Bolkestein of the Liberal Party. Bolkestein made a series of statements at this time that triggered, unwittingly, the first broad national debate in politics and the media about immigrant integration. At an international conference for liberal parties and later in several newspaper articles, he called for a stricter and more 'courageous' approach towards immigrant integration. ${ }^{50}$ Such an approach should be based on the fundamental principles of a liberal society, such as the separation of church and state, freedom of expression, tolerance and non-discrimination. These principles were non-negotiable. It is here that, according to Bolkestein, 'the multicultural society meets its limits, that is, when abovementioned political principles come into play'. ${ }^{51}$ Bolkestein was particularly sceptical about the compatibility of Islam and liberal values. He believed that cornerstone liberal values would have to be defended against immigrant cultures, especially against Islam 'not so much as a religion, but as a way of life'. 52 In this rejection of cultural relativism, Bolkestein explicitly referred to the 1979 WRR report, in which it had been claimed that in cases of confrontation where compromise is practically impossible, there remains no other option but to defend our cultural against competing claims'. ${ }^{53}$

Bolkestein's way of defining immigrant integration was a blend of universalist and assimilationist thinking. On one hand, he seems to adopt a universalist definition of integration in terms of promoting a logic of equity (Mariën 1992: 10) and claiming universal value for specific liberal principles. In this respect, he also referred to the French Republican definition of 
integration. ${ }^{54}$ On the other hand, his specific questioning of the relation between Islam and immigrant integration reveals a more assimilationist framing, directly linking these liberal principles to European history and civilisation. This combination of universalism and assimilationism reflected a shift from neo-liberal to more conservative-communitarian thinking within the Liberal Party of the early 1990s (Fermin 1997: 92).

Thus, a frame conflict emerged at the end of the 1980s, in which the decade's dominant multicultural model was challenged by an alternative frame. Involved here, too, was a questioning of the prevailing technocratic symbiosis, involving actors such as the Department of Home Affairs and ACOM. This came from new actors from both the fields of research and policy. WRR published a second report on immigrant integration that must be seen as disconnected from its first. In addition, there was a growing involvement of research institutes, namely SCP, that produced more quantitative data. Furthermore, Entzinger and Van der Zwan published an influential report that contained strong universalist elements. Finally, political actors became more outwardly involved in policymaking during this period, including Prime Minister Lubbers, who adopted a multiculturalist and universalist frame, and opposition leader Bolkestein, who sparked a broad national minorities debate by making statements that reflected a universalist as well as an assimilationist framing.

This frame conflict involved different ways of selecting and interpreting contextual evidence about developments in immigrant integration. Actors with multiculturalist frames referred to policy stagnation in social-economic domains (e.g. rising unemployment levels) as a sign that the current approach should be continued to prevent the economic recession from having disproportionate effects on minorities. Contending claims that the multiculturalist approach had failed were interpreted as 'impatience', as the emancipation of minorities would take several generations. By contrast, actors with universalist frames did not select or interpret evidence in a way that legitimised the prevailing approach; instead, it signalled that a different one was needed. Ongoing immigration, which created more diverse, larger target groups, and signs of structural social-economic deprivation among migrants were enough to evince the need for a more universalist approach to integration. Furthermore, universalist concerns about social-economic participation were conflated with concerns about social cohesion, as the structural social-economic deprivation of migrants would create solidarity with migrants at risk. From this perspective, the disproportionate effect of economic decline on immigrant unemployment was problematic. More worrisome, however, were the effects that relatively high levels of unemployment among migrants, in comparison to natives, could have on social cohesion and viability of the welfare state. 


\subsection{The construction of enlightenment}

A reconstruction of the research-policy nexus would be central in this frame conflict between new and established actors. Once again, however, research-policy dialogues would be key for a frameshift in this domain, though in a very different way than a decade earlier.

\subsubsection{The field of scientific research}

In the field of scientific research, new actors emerged to define researchpolicy relations in an unprecedented way. Among them was WRR, which, in the course of a decade, had transformed significantly in terms of its problem framing and organisational composition. In fact, as we shall see, a reconstruction of the research-policy dialogues would be a crucial first step towards the frameshift that was codified several years later.

\section{WRR: Anti-establishment boundary work}

Whereas the first WRR report in 1979, 'Ethnic minorities', was deliberately associated with ongoing research and policy developments at that time, the follow-up 'Immigrant policy', was meant to deviate from both. In this respect, boundary work was crucial for contextualising the report and anticipating its impact on ongoing policy developments.

The formal advisory request issued by the government in 1987 had an important effect on the demarcation of research and policy in this period. WRR and the Department of Home Affairs negotiated this advisory request before it was formally issued. ${ }^{55}$ In fact, the advisory request was triggered, if not 'provoked', by WRR itself. ${ }^{56}$ This was facilitated by the personal networks of Albeda, as WRR chairman and chairman of the report's working group, and Entzinger, now a WRR staff member who would be the report's main author. Albeda had been a minister and was a member of the CDA, the same political party to which both the Minister of Home Affairs and the Prime Minister belonged. Entzinger had a strong network on both sides of the research-policy nexus because of his experience at CRM and as ACOM secretary and also because of a recent appointment as professor of multi-ethnic studies at Utrecht University. Also of note is that Entzinger maintained close contacts with the Cabinet, as the Prime Minister formally issues advisory requests to WRR. ${ }^{57}$

The request already specified the substantial areas on which WRR was to focus and what its role should be in discussing policy measures in these areas. WRR would have to concentrate on 'material areas' in which, as the text concludes, too few results had been achieved. It was in these spheres that government 'had doubts whether the current approach should be continued'. This demarcated the focus of WRR from that of the established minorities policy, which also contained a clear 'non-material' focus on 
social-cultural issues. Furthermore, WRR's task was defined as providing 'creative and practical suggestions' to government, aimed at changing 'the prioritisation of government policy' in 1990. This meant that the report would have to be completed within two years.

The formulation of the advisory request led to a boundary struggle concerning the relation between WRR and the government. Objections were raised about the narrow focus on material areas and the limited timeframe that was proposed to complete the request. It was argued that in order to be able to provide scientific advice, WRR should at least be able to put the research problem into a broader context. WRR would have to do 'more than just deliver a toolkit; broader reflection is required, involving a more general report and a longer advisory period'. ${ }^{58}$ Central concepts such as 'minorities' were to be problematised, and it was argued that the focus should be not so much on integration policy but rather, on integration as a social process so that, for instance, value conflicts could also be addressed. ${ }^{59}$ A broader scientific approach would also require a longer time span than the eighteen months that were granted. Otherwise, there would be little opportunity for the report to reflect critically on current policies. ${ }^{60}$ Consequently, some minor changes were made to the advisory request's text. In particular, a phrase stating that WRR would advise on 'minorities policy' was replaced by 'policy with regard to ethnic minorities', thus putting the study into a wider perspective. ${ }^{61}$

WRR interpreted the advisory request, with its particularised task description, rather broadly, including the focus on material needs. Such domains were contextualised within the larger scope of developments in immaterial domains. The council stressed that 'an effective integration policy in these fields [education, labour, housing] would largely obviate the need for specific measures to assist immigrants in other fields' (WRR 1989: 7). Instead of providing advice clearly concerning material areas and only coming up with 'creative and practical suggestions', WRR ultimately developed a new policy frame.

Besides defining its role through - interpretation of - the advisory request, WRR employed a specific mode of discourse to distinguish between this report and the minorities policy. The concepts of 'integration policy' and 'allochthonous' were coined, emphasising the difference between 'minorities policy' and 'minorities', respectively. The concept of an 'integration policy' emerged in a debate on a draft text of the report, questioning whether the extent to which the report would call for policy change had been made sufficiently clear. In order to clarify the fact that WRR would propose a fundamental turning point, it was suggested that the term 'integration policy' be used. ${ }^{62}$ In addition, 'allochthonous' was employed to highlight the fact that the discourse differed to that regarding 'ethnic minorities'. WRR wanted to alter this focus on groups and cultures 
associated with this concept and instead emphasise the social-economic participation of individual immigrants, or individual 'allochthones'.

The definition of ethnic minorities meant that only particular groups were recognised as ethnic minorities, such as the Moluccans, but not the Chinese. Second, it was not the intention to create ethnic minorities in this country, or to fix them to the term 'ethnic minorities'. We actually did not want them to become or remain ethnic minorities, but to become citizens of Dutch society. Basically, the only relevant difference is that they were people that came from different places; hence, allochthones. [...] As such, it was a useful analytical and, above all, more neutral term. ${ }^{63}$

In terms of boundary discourse, the report's literary style became an object of considerable discussion within WRR. On several occasions when discussing draft texts, council members raised objections to the report, arguing for a more 'down-to-earth and empirical style of writing'; a 'less normative' and 'more down-to-earth tone'. ${ }^{64}$ Therefore, a sharper distinction had to be made between analytical observations and normative policy recommendations, for instance, by leaving out phrases such as 'the council thinks that'. ${ }^{65}$ This is a clear example of the type of boundary discourse that Gusfield (1976) describes as the 'literary rhetoric of science'. Choosing a more sober, empirical style and tone would serve to underscore the report's objective, scientific status.

WRR not only defined its report apart from the established minorities policy, but also from established minorities research. In fact, one of the report's aims was to challenge the dominant focus on immigrants as 'minorities'. According to Entzinger, this term would have been 'too unreflectively adopted from American sociological discourse, with too little notion of its application in the Dutch situation and its reifying effects on minorities themselves' ${ }^{66}$ Hence, WRR proposed instead use of the concept 'allochthonous', which was believed to have less reifying effects on minorities as groups because it categorised migrants as 'not from here', rather than in more substantial cultural or ethnic terms. ${ }^{67}$

Furthermore, WRR took a more interdisciplinary perspective than was customary in the field of immigrant integration research; it placed immigrant integration in a broader scientific perspective. ${ }^{6}$ In doing so, WRR put itself at odds with the established anthropological habitus in this domain, as well as with the research methods (field research) and ethos (specific engagement with minorities) associated with this habitus. In contrast, it adopted a more structural-functionalist perspective to immigrant integration, linking it to participation in societal institutions (instead of engagement with minorities) and adopting different methods such as desk and survey research (instead of field research). 
Entzinger sees the interdisciplinary composition of WRR as an explanation for why it could raise new issues that were previously taboo, including a more obligatory approach to immigrant integration. As a professor of migration studies, with a background in sociology and welfare state research, Entzinger was the only member of the project team who was a field-specific expert. ${ }^{69}$ Albeda, the project chairman, had experience as an economist and was a professor of social-economic policy. ${ }^{70}$ Another council member with a legal background was a significant contributor in strengthening the universalist perspective of WRR. He objected to initial texts that called for a 'multiculturalism policy' aimed at the cultural sphere in addition to the 'integration policy' aimed at the social-economic sphere. From a legal perspective, he objected to a policy that would involve 'the removal of present material objections for experiencing positive fundamental rights in the domain of culture' that would be specific to minorities and connected to race. ${ }^{71} \mathrm{He}$ considered this a violation of the legal principle of substantive neutrality with regard to cultures of specific groups and, as such, a violation of the neutrality of the rule of law. The council member used his competence to issue a 'minority position', asserting pressure to change this element of the report. Eventually, the plans were reformulated into a more modest 'culture policy' that would not apply to specific ethnic or cultural groups but to society as a whole - hence, a 'generic culture policy'.

The demarcation of WRR's role from the established minorities policy and research had to do with how the council coordinated its relations with both fields. WRR intended to break into the established structure-induced equilibrium in policy and research. In terms of the former, its aim was not to provide 'creative and practical suggestions' for adapting the minorities policy; instead, the council interpreted the advisory request in a way that allowed it to raise a fundamentally new policy perspective. ${ }^{72}$ To achieve this, it used the concepts 'allochthonous' and 'integration' to signal it was proposing something new. Furthermore, the fact that this report was issued in response to an advisory request also created commitment for policy change based on this report. A formally requested report would be more difficult to ignore. ${ }^{73}$

The fundamentally new perspective on integration that WRR wanted to develop was based on its policy agenda regarding welfare state reform. In this period, under the influence of chairman Albeda (who was still a professor of social-economic policy at the time), WRR developed an agenda for the development of an activating welfare state. ${ }^{74}$ In a newspaper article that followed the publication of this WRR study, Albeda clearly established a link between the 1989 WRR report and this general agenda: 'our welfare state renders individuals too apathetic, and has not been activating enough [...] in the report, the council develops the contours of a more activating welfare state $[\ldots]$ that offers specific rights, but simultaneously also has a more obligatory character'. ${ }^{75}$ In this respect, there was also a clear relation 
between this report and several other WRR reports from the same period, including 'An active labour market policy' (1987a) and 'Work in perspective’ (1990).

The idea for this report emerged in the context of a debate we had about the welfare state. [...] We observed that the problem of immigrant policy suffers from all the shortcomings of our welfare state. I think that this has been one of the most important innovations of the 1989 report, that it simultaneously addresses the problem of the welfare state and shows that the issue of immigrant integration is related to the problems of our welfare state. So, this [perspective] does not stand on its own, but is connected to the perspective we developed in the context of that council. ${ }^{76}$

Another facet of the agenda, related to the first, is that WRR believed the 1979 report had been misinterpreted. ${ }^{77}$ Several project group members found that policymakers had given too little credence to the recommendations on social-economic participation made in the first report and had put too much emphasis on social-cultural emancipation. According to its main author, Entzinger: 'if you take the first report as the middle of the road, then the minorities policy goes in one direction and the second report goes in the other direction'. ${ }^{78}$ With a new report, posited as successor to its first report, WRR hoped to correct this misinterpretation. ${ }^{79}$ As every WRR council only has a mandate for five years, no council can be held accountable for studies produced by its predecessors. However, there was a clear sense of institutional involvement with how earlier reports were translated into policy. Hence, the working title of the second project was initially 'Ethnic minorities II'. 80

Furthermore, WRR wanted to go against the research establishment, which would have focused too specifically on minorities, rather than putting the position of migrants in the context of broader developments in the welfare state. Research would have focused too heavily on migrants as 'the underdog', thereby insulating this topic from broader scientific debates about how migrants could be activated, for instance, in the field of welfare state sociology, areas in which both Albeda and Entzinger had been involved.

Thus, the boundary work of WRR, in terms of demarcation and coordination, was aimed at breaking the structure-induced equilibrium in research and policy. It clearly defined its task apart from minorities policy and research (with the advisory request, in its discourse) in such a way that relations with these fields stressed its fundamentally new perspective (an activating approach, being interdisciplinary). This means, of course, that it challenged the involved actors' status in this structure-induced equilibrium, including the Department of Home Affairs and ACOM. 


\section{ACOM: Expulsionary boundary work}

As previously observed, ACOM weakened in the second half of the 1980s. The WRR report of 1989 further challenged ACOM in terms of its structural position and the ways it demarcated immigrant integration research and coordinated policy relations. Whereas WRR had cooperated closely with ACOM in developing its 1979 report, this time around ACOM was excluded from government plans for an advisory request to WRR and the actual WRR report. This led to a period of difficult relations between ACOM and the Department of Home Affairs. ${ }^{81}$

Following publication of the 1989 WRR report, ACOM believed its duty was to comment on the scientific foundation of this report. Since its duty was to "pay attention to the scientific value of research and the value of research for government policy', ACOM considered it legitimate to write a report on 'the scientific argumentation of the WRR advice' as WRR had 'founded its advice for a large part on scientific research' (ACOM 1989). In the report 'A better policy?', ACOM was highly critical, denouncing the WRR report as insufficiently scientific:

[ACOM] is not positive about its [the 1989 WRR report's] scientific value. [...] ACOM regrets that no clear separation was made between the presentation of scientific material, analysis and policy recommendations. Now all these components are intermixed and normative claims and analysis are indistinguishable. Therefore, it is more a report inspired by science than a scientific report. (ibid.: 25)

ACOM was also critical about WRR's rights-and-duties perspective. It pinpointed a 'biased focus on the individual level and for factors in the sphere of [individual] acquisition of social positions', rather than on the attribution of social positions. It argued that there was too little attention 'for issues such as discrimination and social marginalisation', and a certain 'bias in recommendations [...] on sanctions for members of ethnic groups that would be administered more often than sanctions for employers'. WRR was accused of measuring with two yardsticks - focusing more on the duties of immigrants than on the duties of Dutch society, including government and employers. In other words, it focused too narrowly on deficiencies on the part of migrants rather than structural factors in society at large, or too much on 'position acquisition' rather than 'position attribution' (ibid.). ACOM also argued that the claim that 'minorities have been regarded too much as welfare categories and have become too dependent on government care $[\ldots]$ is an unproven assumption [...] not empirically founded [...] and seems to be inspired by a more general opinion on the welfare state' (ibid.: 25-26). This disagreement revealed differences in the underlying agendas of ACOM and WRR, with the latter holding onto the premise of the minorities paradigm and the former adopting an activating welfare state agenda. 
The WRR report also received criticism on its first report by the author of the influential preparatory study, who was now an ACOM advisory member. Together with another researcher, he published an article in the leading Dutch journal Migrantenstudies, called 'Footnotes to the scientific value of the WRR report' ${ }^{82}$ (Muus \& Penninx 1989). He concluded that WRR had used 'a biased problem definition' and had 'made insufficient use of available scientific knowledge'. Van Amersfoort (1991: 32) also published an article around this time, in which he agreed that the "change in perspective of WRR is not based on scientific arguments or research results, but rather on policy arguments'. Furthermore, several researchers took their boundary struggle about proper immigrant science to the media, stating among that the WRR report 'contained small talk'. ${ }^{83}$

This institutional clash also led to a personal struggle between Entzinger, the main author of the 1989 WRR report, and his colleagues at Utrecht University, including ACOM chairman Bovenkerk. In 1990, a conflict arose over a professorship in the research group on studies of the multi-ethnic society (SMES). This position was awarded to Entzinger, much to the chagrin of some colleagues. The appointment spurred a revival of the controversy surrounding the 1989 WRR report and media statements made by Entzinger concerning the permanent nature of immigration. ${ }^{84}$

This conflict showed that the institutional and personal struggles, both, were also about different views on how to demarcate and coordinate research-policy relations. Entzinger's colleagues wrote a letter to the faculty dean, asking for his resignation due to a lack of 'intellectual leadership', 'insufficient theoretical knowledge of the research field' and 'lack of research qualities'. ${ }^{85}$ Among other shortcomings, Entzinger was said to have had too little experience and knowledge of the type of field research possessed by most of his colleagues, making him incapable of leading this research group. This focus on field research reflected the demarcation of immigrant integration research advocated by ACOM throughout the 1980s.

In terms of coordination, the combination of being a professor as well as a WRR staff member would have created a significant dilemma for Entzinger in terms of both scientific independence and what was described as being a 'civil servant'. ${ }^{86}$ Entzinger was attacked for having a 'strong sensitivity to public opinion and policymakers' that, critics argued, had been demonstrated by the 'strong, tough and firm statements' he had made in the media. ${ }^{87}$ In short, his work for WRR and his professorship at Utrecht University were considered incompatible with one another. Moreover, Entzinger's academic colleagues had concerns about the effects of his statements in the media and his involvement in the 1989 WRR report on the position of migrants. Such statements would 'not have been directed at combating the hostile mood toward migrants or the combating of prejudice' and would, in some cases, even 'have reinforced this mood'. ${ }^{88}$ This illustrated the discrepancy between the focus of these researchers on 
issues such as anti-racism and factors in Dutch society that inhibited emancipation, rather than considering those factors on the side of immigrants that hampered their integration. ${ }^{89}$ Moreover, such media statements were considered potentially harmful to the relations between the involved researchers and the minority groups being studied..$^{90}$

In the months after the WRR report and the letter to the dean, fruitless attempts were made to reunite this research group. In early 1990, this conflict was revealed to the national media, even appearing on the front page of some leading Dutch newspapers. ${ }^{91}$ Eventually however, the dean decided to dismiss Entzinger's colleagues at Utrecht University and to dissolve the SMES research group. ${ }^{92}$ Entzinger maintained his professorship.

ACOM thus adopted an expulsionary mode of boundary work, attempting to counter WRR's denunciation that it was unscientific. It re-emphasised its demarcation of immigrant integration research by engaging with the specific position of minorities (also in terms of methods) and coordinated research-policy relations in a way that involved more distance from policy. However, the distancing from ongoing policy developments, which were to a great extent the consequence of the WRR report requested by the government, and its failure to respond to growing demands for more evaluative expertise, gradually undermined ACOM. The controversy surrounding the 1989 WRR report contributed further to this process. ${ }^{93}$ In 1992, the mandate for ACOM was eventually withdrawn.

\section{SCP: Boundary work of a data provider}

Apart from occasional attention for immigrant integration in several documents from the 1980 s, SCP paid more structural attention to immigrant integration from the early 1990s onwards. In annual and, later biannual, minorities reports, SCP provided data on the status of immigrants in various areas, primarily social-economic ones such as education and labour. These minorities reports were the product of a contract between SCP and the Department of Home Affairs.

SCP demarcated its role in terms of the provision of quantitative data. It made secondary analyses of data from the Statistics Office (CBS). ${ }^{94}$ This concerned generic data, or data that was derived from general databases such as the CBS Labour Force Survey (VWJ 2004: 76-77). The data on ethnic minorities were derived from these generic databases. SCP did not use specific methods for reaching migrants to gather this data about ethnic minorities. Only later, in the 1990s, would SCP cooperate with the Institute for Social and Economic Research (Instituut voor SociologischEconomisch Onderzoek, ISEO) that did employ specific techniques for obtaining data on immigrant minorities (Meloen \& Veenman 1990; VWJ 2004: 79). ${ }^{95}$ Thus, SCP's task was defined as providing 'hard facts' on the social-economic position of migrants. 
In terms of coordination, SCP was to be both instrumental and functional to policy departments. ${ }^{96}$ Evaluations of the status of minorities in various areas would enable government departments to assess the effectiveness of their approach, and to adapt if necessary. Furthermore, the contract with the Department of Home Affairs gave this department an important say about the domains that SCP should focus on. In this respect, SCP's mandate was government demand-driven.

SCP was careful not to venture beyond its instrumental role in this period. It did not repeat criticism on multiculturalism from its 1986 'Social and cultural report' until the publication of 1998's version. However, Van Praag, one of the leading researchers at SCP responsible for the studies on immigrant integration, continued to criticise multiculturalism in his columns for the scientific journal Migrantenstudies. He published under the pseudonym Vyvary, so as to be able to speak freely and to avoid the risk that his statements would be taken for views of SCP (Van Praag 2003: 74). ${ }^{97}$

\section{Van der Zwan and Entzinger: Entrepreneurial boundary work}

Both Van der Zwan and Entzinger were known for advocating a different approach to immigrant integration. As a member of the WRR council that developed the 1979 report on ethnic minorities, Van der Zwan had already advocated a more obligatory approach in which labour would be the main channel for integration. ${ }^{98}$ Entzinger, as the main author of the 1989 WRR report, had proposed a similar activating approach to the social-economic participation of migrants. The national minorities debate that emerged in 1992 following a number of statements by Bolkestein led to a broader public and political acceptance of a more activating approach to immigrant integration primarily by means of labour and education. However, this had not produced concrete policy changes in this direction. In this respect, Van der Zwan and Entzinger would be invaluable entrepreneurs in translating this approach into concrete policy measures.

Van der Zwan and Entzinger defined their roles in a way that stressed the political and policy relevance of their advice. Their aim was 'not to come with lengthy analyses, but with concrete proposals that could be realised by politics'. 99 Furthermore, these concrete proposals would have to be practically feasible. To make sure the proposals could be put into practice, the report was subjected to debate in two mini-conferences with various actors involved in policy practice. As they themselves put it:

this way, we not only made sure that our plans were legally and economically feasible but also that there was a certain public support for it, especially among those who were directly involved in the practice of immigrant integration. (Van der Zwan \& Entzinger 1994: 1) 
Furthermore, political support was generated by involving various political parties in the making of the report. Minister of Home Affairs Dales and Van der Zwan were both members of the Social Democratic Party. Director General of the Department of Home Affairs Van Aartsen, who at that time had an important role in requesting that Van der Zwan and Entzinger write the report, was a member of the Liberal Party. In addition, Entzinger was a member of the Liberal Democratic Party. One of the reasons for involving these three political parties was that it was assumed that they would be involved in the formation of a new government coalition in 1994.

During the preparation of our proposals, we immediately looked at the support it would raise. Endless debates were held to estimate how the plans could be received. Entzinger was involved because it was thought that D66 [Liberal Democratic Party] would be involved in the next formation. I was involved because of my affiliations to the PvdA [Social Democratic Party]. The aim was to achieve a certain acceptance. ${ }^{100}$

In addition to this orientation on public and political support, it was clear that Van der Zwan and Entzinger shared ideas about a more obligatory approach to immigrant integration and that both focused on labour as the means for achieving integration. They shared a more or less similar frame. ${ }^{101}$ In this respect, their way of demarcating their tasks from established immigrant integration reflected that of the 1989 WRR report. Both resisted the tendency to ignore the 'contraindications' that illustrated the deficiencies of the multiculturalist approach. ${ }^{102}$ Van der Zwan noted:

We knew each other from WRR networks: it is like a family. I strongly agreed with the tone of the second WRR report [...]. The report also states clearly that we were unhappy about the practical consequences that had been given to that WRR report. So, that was the motivation. We could not name it 'Policy succession WRR report', because then it would be too much of WRR and because we had already had the minorities debate in between. So, there was a clear link between the second WRR report, the minorities debate and the report 'Policy succession minorities debate'. ${ }^{103}$

In terms of coordination, the Van der Zwan and Entzinger report directly aimed to influence the coalition formation of 1994. There was a belief that the minorities debate and the report by Van der Zwan from 1992 still had too little impact on concrete policy measures. This set the tone for top administrators from both the Department of Home Affairs and the Minister of Home Affairs to seek advice. It was also the backdrop against which Van der Zwan and Entzinger got involved. The parliamentary elections that 
were held in May 1994 would provide a window of opportunity for achieving more concrete policy effects. This was especially so because of the predicted decline of the Christian Democrats, who had led government since 1982 (three cabinets led by Prime Minister Lubbers), and had been consistently involved in government since World War II. The aim was to have an advisory report that could be used during the formation of a new government coalition in 1994, hence the timing of the publication of the report, May 1994. Furthermore, Van der Zwan and Entzinger were in close contact with the leaders of the various political parties during these talks to form the coalition (Van Thijn 1994: 256-257). ${ }^{104}$

Thus, different boundary work practices were found for different actors in the research field in this period. WRR strategically demarcated the role of its report from the established minorities policy, as well as research to develop a fundamentally new perspective on immigrant integration for both fields (as part of its broader agenda on welfare state reform). Van der Zwan and Entzinger also tried to achieve a breakthrough in the structureinduced equilibrium of multiculturalism through a more entrepreneurial sort of boundary work. They saw WRR as being meant to ensure feasibility of the new approach and thus coordinated it directly with ongoing political developments, such as the coalition formation in 1994. In contrast, ACOM responded to the 1989 WRR report with a more expulsionary sort of boundary work, holding on to the prevailing way of demarcating minorities research, coordinating its relations with policy and minority groups and denouncing the document as 'unscientific'. Evidently, these boundary struggles over the proper division of labour between research and policy were closely related to the frame conflicts discussed earlier. This clearly underscores Gusfield's (1976) premise: the culture and the structure of public problems must always be seen as inherently connected.

\subsubsection{The field of policymaking}

Boundary struggles in the field of migration between new and established actors were also manifest in the field of policymaking. Once again, the field of research would provide a venue for policy change. However, this time it did not involve rational societal engineering 'behind closed doors', as had been the case a decade earlier. Rather, it would involve the agenda setting of new ideas about immigrant integration through authoritative venues of research that the status quo would find difficult to ignore.

\section{Department of Home Affairs: Changing position, changing boundary work}

As coordinating department, the Department of Home Affairs was involved in the established research-policy nexus from the early 1980s. However, this changed in the late 1980s and early 1990s, when the department lost 
its coordinating power due to the functional and territorial decentralisation of the integration policy.

The advisory request to WRR in 1987 was a first sign that the changing position also led to this actor's changing boundary work practices. The Minister of Home Affairs sought new recommendations from WRR in response to mounting pressure in Parliament regarding the department's coordinating role. On one hand, the minister felt pressure to fulfil coordinating duties in the face of inadequate policy effectiveness in mainly material areas. ${ }^{105}$ On the other hand, he experienced difficulties in achieving interdepartmental coordination, as various departments increasingly chose to follow their own policy lines, in part because of huge budgetary constraints on them. ${ }^{106}$ This tension surfaced in 1986 when disagreement emerged in Parliament about what appeared to be inconsistencies in the budgets presented by the coordinating minister and those presented by the departments themselves. ${ }^{107}$

There was an agreement between government departments that there would be no budget cuts for the minorities policy. Nonetheless, disagreement emerged about changes in departmental budgets that seemed to reveal cutbacks on measures taken in the context of the minorities policy (education and social affairs, among others) (Koolen 2003: 25). Although the coordinating minister denied that any budget cuts would take place, this disagreement revealed the heightened expectations for the coordinating role of the Minister of Home Affairs and the alleged difficulties of interdepartmental coordination. ${ }^{108}$ The minister faced further constraint on his scope of action because of the norm that this should be kept a non-partisan issue. ' $[\mathrm{T}]$ here was a sort of unwritten code between political parties not to engage in debates [...] that could lead to stigmatisation of minorities'. ${ }^{109}$ Together with the problems of interdepartmental coordination, this constraint made the annual debates on the action programmes for the minorities policy into 'annual rounds of beating up on the Minister'. 110

In response to this political pressure and constraints on interdepartmental coordination, Minister of Home Affairs Van Dijk decided to issue an advisory request to WRR. As discussed earlier, this advisory request was formulated together with the Minorities Policy Directorate - still led by Molleman - and with WRR itself. The advisory request demarcated an interest in a specific type of expertise, specifically on integration in material areas. According to Van Putten (1990: 366), research manager of the Minorities Policy Directorate at that time, the department had an interest in more evaluative research about what kind of approach works and what does not work. This demand for more evaluative expertise was a consequence of the ongoing process of institutionalisation of the minorities policy. Now that the minorities policy had been formulated and had entered the implementation stage, there was more demand for knowledge concerning the effectiveness of policy measures, rather than fundamental questions 
concerning problem framing. In this respect, ACOM would have been left behind in terms of responding to this changing demand for expertise. For instance, ACOM failed to respond to the government's growing demand for research that could monitor the progress of integration in specific domains, such as the accessibility and proportionality project on monitoring minority participation in mainly social-economic spheres, which was in fact eventually given to a new research institute (the ISEO) without consulting ACOM in this decision. ${ }^{111}$ Whereas ACOM had been closely involved in establishing the minorities policy, it now became more marginal. In the late 1980s, the Department of Home Affairs would start to play a more active role in research programming. In fact, ACOM was not even involved in, or notified about, the advisory request to WRR until a relatively late stage (Van Putten 1990).

In addition, the advisory request held a specific idea about the report's coordination with ongoing developments, namely that it should provide corresponding suggestions for new government policy. The advisory request offered a clear political statement about the need for a different approach. This brought the current policy coordination structure to crisis, ${ }^{112}$ invigorated when WRR, having interpreted the advisory request with broad strokes, produced a fundamentally new policy perspective in its 1989 report, one which also addressed the Minorities Policy Directorate. Instead of having a clear-cut coordinating duty, this directorate would only monitor the contours of the integration policy.

The position of the Minorities Policy Directorate changed significantly in the late 1980s. For one, this was symbolised by renaming it the Directorate Contours of the Minorities Policy. Following the 1989 WRR report and the departure of director Molleman, the coordinating task was formulated further in 1991. ${ }^{113}$ Policy responsibility was now located more clearly with the various departments responsible for specific programmes in their sectors. In addition, the role for a severely downsized Directorate for the Coordination of the Integration of Minorities (DCIM) would be to coordinate the measures taken in various domains by means of monitoring of policy results (Koolen \& Tempelman 2003: 90). ${ }^{114}$

The changing position of the Department of Home Affairs involved another way of demarcating and coordinating research in policy formulation than had been customary in the established research-policy nexus of the early 1980s. Instead of research that provided expertise on the status of minorities to aid policy formulation, it now had a more specific interest in more evaluative studies that could be used to assess policies conducted in various policy sectors. It is in this context that the Department of Home Affairs came to an agreement with SCP regarding regular minorities reports. In terms of coordinating research-policy relations, this data on the social position of minorities in various policy sectors (particularly labour, education and housing) was crucial in the Department of Home Affairs' 
interdepartmental coordination of the integration policy. It allowed this department to evaluate results in a number of policy sectors and appeal to the responsible departments to take appropriate policy measures. ${ }^{115}$ Furthermore, through the open publication of the data gathered by SCP, the Department of Home Affairs could put issues on the agenda more easily and encourage policy measures through this venue. The director of DCIM declared:

At the department, we reflected on what we wanted to know based on the policy goals. [...] This concerned the groups about which we want to know things, involving a limited number of target groups [...]. This also concerned what we wanted to know about these groups in terms of their position in labour, education and housing [...]. We wanted to have two types of studies, one annually about where we stand at that moment and biannual in-depth studies of specific topics. [...] This means that, for instance, when the department of education says it is doing very well with education for minorities [...] but research initiated by our department showed that this was not the case, that this issue could then be put on the political and public agenda [...]. Consequently, it would be reported to Parliament or written about in the media, so that the Minister of Education and his civil servants could be held accountable [...]. This meant that we organised our information position based on ideas about what precisely we wanted to know for which groups [...]. This has proven to be a very effective coordination mechanism. $^{116}$

The request for recommendations from Van Der Zwan and Entzinger on the policy succession of the minorities debate also served a strategic purpose for coordination carried out by the Department of Home Affairs. The national minorities debate created a renewed political sense of urgency about immigrant integration and the conferences held by the Department of Home Affairs led to ideas for development of civic integration programmes. According to the director of DCIM:

The Department of Home Affairs found itself in a difficult position; it was responsible for coordination but lacked the appropriate means. So we had been thinking, who can we ask to give advice with real pragmatic meaning, as much has already been written. [...] And we found that they had the quality - Entzinger the substance and Van der Zwan also the means for presentation. [...] So, it really had to do with strategic positioning. What did not succeed with the WRR report, and the government reply memorandum was getting the financial means for a civic integration policy. ${ }^{117}$ 
By identifying this advice as a provision of concrete policy proposals that could lean on both sufficiently broad support and Van der Zwan's and Entzinger's support, the department hoped to coordinate ongoing political developments (such as coalition formation in 1994) in such a way as to provoke concrete policy choices. This particularly concerned the idea of civic integration programmes, which the Department of Home Affairs wanted under its own provision as departmental coordinator. Up until then, the courses were provided on a limited scale by CRM. Entzinger and Van der Zwan's report, then, was a strategic initiative to bring these courses under the remit of the Department of Home Affairs. ${ }^{118}$ Indeed, following the coalition formation of 1994, civic integration programmes were developed by this department and, during the late 1990s, would grow into one of the most important facets of the integration policy.

Thus, the boundary work of this department changed significantly. In contrast to its role in the established research-policy nexus of the early 1980 s, its way of demarcating and coordinating research-policy relations changed in the second half of the decade. It demarcated a more specific interest in evaluative research, which was coordinated to policy development in more instrumental and functional terms. This changing boundary work is particularly manifest in its relations with SCP, but also in the advisory request to WRR, though WRR would eventually demarcate and coordinate its role separately. Finally, the minorities debate in the early 1990 s seems to have given rise to more entrepreneurial boundary work by the Department of Home Affairs, in an attempt to strengthen its coordination mandate vis-à-vis the increasing sense of urgency and changing public and political discourse.

\section{Politics: The boundary work of politicisation}

In the early 1990s, political actors became more outwardly involved in public debates about immigrant integration. Notably, the minorities debate of 1992 created a widespread sense of political urgency. However, prior to this debate, the Prime Minister had made public statements that hinted at a different approach to immigrant integration, reflecting the recommendations of the 1989 WRR report. Fermin (1997: 211) observed a rather general shift from emancipation to integration in political discourse in this period. There was also common agreement about the need for additional policy efforts to achieve social-economic participation (ibid.: 227). Political differences persisted, mainly on social-cultural issues, though they gradually shifted to the background.

This politicisation involved a different way of demarcating the tasks of research and policymaking. The technocratic research-policy nexus of the early 1980s involved a demarcation of immigrant integration as a non-political issue that was delegated to researchers and policymakers who would 
develop a rational policy approach based on policy-relevant knowledge and expertise. Bolkestein now clearly defined immigrant integration as a political issue. He stated in the media that he no longer wanted to hide his party's position out of fear that extreme-right parties would play the race card, arguing that 'when a democratically elected politician fails to put this major issue crystal clear on the table, then he is functioning inadequately'. ${ }^{119}$ In this context, he called for the eradication of taboos and for an open debate on this issue, saying:

The integration of minorities is such a complex issue that it can only be resolved with courage and creativity [...]. [I]n this there is no space for lack of engagement and for taboos [...], [A] broad debate in which all political parties participate is required $[\ldots] .{ }^{120}$

Bolkestein's statements, as well as the many responses it provoked, were not just about the issue of immigrant integration, but also about the demarcation of the role of politics in dealing with immigrant integration. Bolkestein wanted to rid political involvement in this domain of the sense of 'political correctness', which implied 'that one could not have the courage to name sensitive issues' as a sort of 'self-restriction [...] that limited one's perception'. ${ }^{121}$

Prins $(1997,2000)$ has described this new mode of political discourse as 'new realism'. This involves, first of all, an eradication of taboos; reality would have to be represented 'as it is', without the burden of taboos or distorting values and interests. This would be a representation of typical Dutch 'virtues', such as honesty, courage and soberness. New realism also claimed to represent the opinion of the 'ordinary citizen' who had thus far been largely ignored. Instead of avoiding debate and conflict, new realism meant engaging in debate with immigrants - a signal that these immigrants are taken 'seriously'. More precisely, this implied treating them as equals and not as dependents; immigrants had become citizens and new realism would appeal to the civic duties of migrants. As such, it reinforced a universalist problem frame that defined immigrants as equals rather than emphasising the specificity of minorities.

In terms of coordination, the politicisation in this period did not mean that scientific research went entirely ignored. Instead, Bolkestein and Lubbers made explicit references to the 1979 and 1989 WRR reports. The one from 1989 also played an important role in the broader public and political debate. In the parliamentary hearing surrounding this minorities debate, the 1989 report was praised for its contribution to the sober and realist tone of the debate and, in contrast to prior publications, emotions did not gain the upper hand (Koolen \& Tempelman 2003: 94). ${ }^{122}$ That said, references to scientific research clearly became more selective in this period. Whereas the 1989 WRR report became a benchmark in public and political 
discourse, other studies (such as the 1986 WRR report 'A fair chance' and its 1989 report 'A better policy?') seem to have been marginal.

Thus, in the field of policymaking, the pattern of boundary work had changed in a relatively significant manner when compared to the preceding period. Instead of demanding policy-relevant knowledge about the status of minorities for policy formulation, the Department of Home Affairs now sought more evaluative knowledge and expertise to strengthen its capacity in interdepartmental policy coordination. Its relations with SCP involved a more instrumental type of boundary work, whereas relations with WRR and, later, Van der Zwan and Entzinger involved a more strategic type. Furthermore, political actors in this period changed their boundary work from defining immigrant integration as a non-political issue best left to researchers and policymakers, to defining it as a political issue - one politicians should feel free to express their opinions about. In this context, scientific studies that reinforced the changing mode of political discourse and the 1989 WRR report, in particular, continued to play an influential role.

\subsubsection{An enlightenment boundary configuration}

Analyses of the fields of immigrant integration research and policymaking indicate that there were evident changes in the patterns of boundary work. Yet, how did these developments in both fields combine to punctuate the technocratic symbiosis that originated a decade earlier? And exactly what sort of research-policy nexus was produced in this period?

\section{Divergence in research-policy relations}

The new episode of research-policy dialogues on immigrant integration that was initiated by the 1989 WRR report 'Immigrant policy' did not directly lead to policy change. The structural equilibrium originating from the 1980s had successfully mobilised negative feedback to hold off immediate policy change. In fact, endeavouring to punctuate this equilibrium, WRR deliberately created more divergence in research-policy relations as compared with the technocratic symbiosis from the 1980s. Consequently, its 1989 report, and particularly the proposed new problem frame, were not directly utilised in government policy. Not only did ACOM respond with expulsionary boundary work, but the Department of Home Affairs did not immediately give effect to the new perspective and the consequences it would have for the coordinating department. Although the reply memorandum adopted various elements of the 1989 document, it did not incorporate some of its most fundamental elements, such as the definition of 'allochthonous' and the rights-and-duties perspective on integration through labour market and education.

The report did have a more significant impact on public and political discourse. It attracted broad attention in the national media, as illustrated 
by the many headlines claiming that WRR had shown that the minorities policy had failed and that the policy 'coddled' minorities. ${ }^{123}$ Several weeks before the WRR report was made public, it was leaked to the press, who immediately picked up on its sense of urgency, and produced headlines such as 'WRR attacks the minorities policy' and 'WRR wipes the floor with the minorities policy'. ${ }^{124}$ Although nowhere in the WRR report did it state that the minorities policy had been a failure, this became the main point taken up in the media. Despite this, most political parties received the report positively, and Prime Minister Lubbers referred approvingly to it when he was one of the first to break the taboos on politicisation in 1990. The report also indirectly effected changes in the coordinating task of the Department of Home Affairs and the development of the DCIM, in particular.

Several years later, the 1989 WRR report received renewed attention during the national minorities debate to emerge in 1992. During this debate, it became clear that the more activating social-economic approach to immigrant integration that WRR had proposed had now become more broadly accepted in public and political discourse. It was in the aftermath of this national minorities debate that policy was reframed according to the universalist perspective put forward by WRR.

Scientific research was involved in this reformulation, but not in a structural way as had been the case in the late 1970s and early 1980s, nor in a way that involved established research institutes such as WRR or ACOM. Rather, individual experts (Van der Zwan and Entzinger) were active entrepreneurs in the diffusion to policymakers and politicians of the perspective that had been developed in the 1989 WRR report. This involved not so much further development of this universalist perspective but rather, the generation of public and political support. It was also an attempt to make use of the window of opportunity of the 1994 coalition formation to have this perspective translated into concrete policy initiatives. This involvement was not structural but rather, an incidental 'coalition of convenience' between the Department of Home Affairs and the experts Van der Zwan and Entzinger who, after the national minorities debate, both shared a similar universalist perspective.

In relation to research, there also seems to have been more divergence than in the early 1980s. Whereas ACOM was central to the provision of policy-relevant knowledge for policy formulation, this institutional nexus was dissolved when ACOM was discontinued in 1992. During the 1980s, as the minorities policy became increasingly institutionalised, government demand for expertise changed. It honed a new interest in more evaluative expertise, which had more indirect relations with policy development. Instrumental data provided by SCP was only indirectly related to policy development as a means for interdepartmental policy coordination. The 
information offered a tool for monitoring policy results across the board rather than a direct means for influencing policy development.

In contrast to this divergence in policymaking and research, there seems to have been some convergence - in terms of personal networks - among those experts involved in the 1989 WRR report and politicians. Like others, Albeda, who served as chair of both WRR and the working group for the second WRR report, had a political background. He was Minister of Social Affairs from 1977 to 1981 and maintained close contacts with the incumbent Prime Minister who belonged to the same Christian Democratic Party. Furthermore, Entzinger, one of the main authors of the 1989 WRR report, maintained close ties with the Minorities Policy Directorate, as well as with the Cabinet. These contacts seem to have been pivotal in formulating the advisory request to WRR. As such, the diverging role between WRR and pre-established research and policy was a product of WRR's deliberate design in the context of ongoing political developments.

\section{Scientific primacy}

There are signs in this period of scientific primacy as well as a modest political primacy. An important indication of the former was the 1989 WRR report's considerable influence on the development of integration policy. Although, as we have observed, it did not immediately trigger policy change, it did catalyse public and political debates that would eventually lead to policy changes. It punctuated the structure-induced equilibrium in both research and policy by making immigrant integration an issue of broader scientific and political debate, thereby creating a setting in which policy change and progress in research could become possible. This involved challenging both the structural position of established researchers (ACOM) and of established policy actors (Minorities Policy Directorate). Furthermore, the influence of WRR on policy developments also concerned the activating welfare state perspective on immigrant integration. Many aspects of the 1989 WRR report were adopted in the contours memorandum of 1994, including its more all-encompassing way of categorising migrants and its causal theory of achieving immigrant integration by activating migrants in the social-economic spheres of labour and education.

At the same time, there was also growing political primacy. The politicisation incited by the national minorities debate reveals how political actors' participation in this domain was intensifying. The debate put immigrant integration firmly on the political agenda, punctuating the norm of depoliticisation in this field. In addition, the duty of SCP and its relation to government since the early 1990s provides some indications of growing political primacy. The Department of Home Affairs gradually took a more active part in research programming during the 1990s, especially in terms of encouraging more evaluative research (Van Putten 1990). Its agreement with 
SCP on providing quantitative data on the position of minorities was a concrete manifestation of policy's influence on developments in research. In this respect, the major role of research actors (ACOM) in research programming made way for stronger politicisation in this area.

The changes in the policy field in the early 1990s seem to have been conditional, depending on the politicisation that occurred during the national minorities debate, following Bolkestein's statements about the need for a more courageous approach towards the integration of migrants. However, when we look at the actual relations between research and policy actors in this period and their influence on policy developments, a more primary role for research emerges. Political actors such as Bolkestein and Lubbers founded their political claims on WRR reports, particularly 1989's. Furthermore, the national minorities debate itself seems to have had little direct effect on policy developments; the political ideas about policy change seem to have remained abstract rather than concrete. ${ }^{125}$ At this stage, Van der Zwan and Entzinger were crucial for the translation of the universalist perspective into concrete policy choices. The influence of the WRR report on policy developments was also manifest in the contours memorandum that was issued by the renewed Minorities Policy Directorate in this period.

Thus, beyond the veneer of politicisation, a more primary role for research emerged. It was WRR that first put immigrant integration on the agenda in 1989, opening up debate at the level of problem framing about a new perspective on immigrant integration and challenging the structural equilibrium in this field. The politicisation after the national minorities debate moved this discussion into the political arena, but did not effectuate specific policy change. It did create a political environment for policy change in which many ideas from the 1989 WRR report were revived. In terms of policy reframing, the changes that were to come in the early 1990s reflected the ideas put forward in the report. In fact, the translation of this new perspective into concrete policy plans was aided by Entzinger and Van der Zwan's efforts.

This analysis shows a divergence flourishing between the task of research and policy at this time. Moreover, there was a modest scientific primacy in their mutual relationship. The effect that the 1989 WRR report had - and its influence on policy developments in the early 1990s reflects a model of gradual enlightenment in government and politics based on scientific research.

The enlightenment nexus was the product of very specific factors within and beyond the field of immigrant integration. First, it was a consequence of the political agenda of WRR, seeking to introduce a new problem perspective as part of its strategy to punctuate the structure-induced equilibrium in immigrant integration research and policy during the 1980s. Furthermore, it was influenced by developments in the broader political 
setting in this period. During the 1980s, a politics of welfare state retrenchment had been conducted in many policy areas, even though for specific reasons - such as the taboos surrounding an activating approach to immigrant integration and the idea that minorities were especially vulnerable because of the overall economic decline - this had not been the case for immigrant integration. Construction of an enlightenment nexus offered a legitimate way to connect this political discourse to immigrant integration and thereby also punctuate the established structure-induced equilibrium in this domain.

\subsection{Enlightenment and frameshifts}

As we have seen, the construction of the enlightenment nexus was the outcome of specific factors within and beyond the fields of immigrant integration research and policy. However, how did this nexus influence the frameshift from multiculturalism to universalism, and to what extent did its role involve critical frame reflection?

\subsubsection{The structural effects of enlightenment}

The enlightenment model comes close to the standard model of 'science speaking truth to power' in terms of research-policy dialogues. Whereas technocracy means that research is very much involved in power, enlightenment strongly suggests that research provides new policy ideas that are to impact policy developments in a more indirect manner. How, then, did this 'enlightenment' function of research-policy dialogues impact on the heavily institutionalised status quo that had evolved in the fields of immigrant integration research and policy from the 1980s?

\section{Enlightenment as a source of positive feedback}

The enlightenment type of boundary configuration had a disturbing effect on the structural symbiosis between established research and policy actors in this domain. In particular, WRR's boundary work was strategically aimed against the established minorities policy as well as research. The universalist approach that framed integration policy as an intensification of general policy - which WRR had put on the agenda - weakened the position of the Minorities Policy Directorate, which advocated a multiculturalist approach to ethnic minorities. It also challenged ACOM's status, which had thus far maintained a central position in the immigrant integration field, by excluding the committee from this WRR project and adopting an individualist and activating welfare state approach to immigrant integration. This approach contrasted sharply with focus on the status of ethnic 
minorities and issues such as discrimination and emancipation, as propagated by ACOM.

As previously observed, these actors involved in the structural symbiosis mobilised significant negative feedback for the 1989 WRR report. ACOM denounced the WRR report as 'unscientific', based on its own demarcation and coordination criteria of minorities research. Furthermore, the Minorities Policy Directorate initially refused to give full credence to the WRR report, arguing that the minorities policy had not failed though had merely not yet succeeded.

The enlightenment boundary configuration was primarily a product of WRR's boundary work and political actors. For WRR, the report constituted a success story in achieving its agenda on welfare state reform. Although it initially led to debates about WRR's scientific status, especially in relation to its breaking alleged taboos on discussing the rights and duties of immigrants, the report eventually boosted WRR's authority in dealing with controversial policy topics (Hirsch-Ballin 1997: 116). For the second time, WRR had issued a document that would mark a turning point in immigrant integration policy.

For political actors, the enlightenment boundary configuration provided a way of punctuating the structure-induced equilibrium that had so long insulated this policy area from broader political developments. The politics of welfare state retrenchment that had already affected many social policy domains now also penetrated immigrant integration policy. Significantly, the enlightenment configuration, supported by WRR's scientific authority, and the experts involved in the 1989 report, provided a legitimate venue for connecting this issue to broader concerns about the welfare state, as the taboos surrounding this topic and the norm of depoliticisation blocked other venues from raising such a perspective. Even when Lubbers and Bolkestein encouraged broader politicisation of this issue, they often referred to WRR as an authoritative source for their political claims.

\section{The negative feedback of a new structure-induced equilibrium}

Thus, the enlightenment configuration, with major involvement by WRR, was successful in changing public and political discourse and altering the roles held among involved actors. With the establishment of the universalist approach, there was growing need for more practical and instrumental expertise that could help construct a new structure-induced equilibrium around it. In the policy field, this involved a structural repositioning of the Department of Home Affairs, in which the roles of SCP, Van der Zwan and Entzinger were of great importance. In the research field, this involved a structural positioning of SCP and, until 1996, TWCM.

The Department of Home Affairs and, in particular, the drastically reformed DCIM, attempted to strengthen its structural position within the new universalist approach by organising its information position in relation 
to other departments. The minorities reports produced by SCP provided an instrument for the interdepartmental coordination of the integration policy. With these reports, the Department of Home Affairs could influence the policies of other departments by presenting data on the position of minorities in areas that fell under the responsibility of specific departments. Furthermore, in the aftermath of the minorities debate, the Van der Zwan and Entzinger report served as an important strategic initiative for bringing the provision of civic integration programmes under the remit of the Department of Home Affairs. This move would prove to have a great effect on the position of this department in the integration policy of the 1990s. However, the department continued to cope with problems of interdepartmental coordination. This is illustrated aptly by a passage in the memoirs of former Minister of Home Affairs Van Thijn regarding interdepartmental debates about the contours memorandum that was to be published in 1994.

The interdepartmental debate on the contours memorandum ends up in a battle. From all sides, severe objections are made. [...] When I see his [Minister of Education's] notes I become very angry. He suggests getting rid of almost all the plans that fall in his domain. [...] The content of the memorandum suffers severely under these debates. The formulations that had made this memorandum so powerful had been severely tuned down. There is no longer question of a re-calibration of policy, which had been asked for [...]. The word 'recalibration' has been systematically deleted. (Van Thijn 1994: 115-120)

In the field of research, SCP now, for the first time, obtained a more central position. During the 1990s, it became the most important provider of data on the position of minorities in various domains. However, no organisation during the 1990s obtained a central position within the field of immigrant integration research that was comparable to the position ACOM had held during the 1980s. For some time, the bipartite TWCM was important for the dissemination of research findings towards policy practices, but it was dissolved in 1996.

Thus, by disturbing the structural symbiosis between established actors in the fields of research and policy (ACOM, Minorities Policy Directorate) and strengthening the position of other actors in this period (WRR, political actors, Department of Home Affairs or DCIM after 1990) the enlightenment configuration provided an important source of positive feedback. The fact that the research-policy nexus was structured according to an enlightenment type of nexus was of great relevance, as this created a legitimate, effective way of punctuating the structure-induced equilibrium from the 1980s, which involved such a powerful symbiosis between specific actors 
and powerful taboos and norms of depoliticisation. When this equilibrium was successfully punctuated, a new structural equilibrium was achieved that involved a more instrumental, strategic role for research (with actors like SCP, Van der Zwan and Entzinger).

\subsubsection{Enlightenment and universalism}

The enlightenment nexus was clearly configured to challenge the multicultural model that had been dominant in the Netherlands in the decade before; it provided the structural conditions for a frameshift. However, did it also contribute to the rise of a universalist frame in particular? Did it also challenge the 'logic of minorities' that had supported the multiculturalist frame in the 1980s?

The integration policy: From the logic of minorities to the logic of equity An important consequence of the enlightenment nexus was that immigrant integration was now, finally, connected to broader societal issues, particularly concerns in policy and politics about the welfare state. Thus, the politics of welfare state retrenchment that had affected many social policy sectors in the 1980s, but from which the field had been successfully insulated, now penetrated immigrant integration policies as well. This issue linkage meant that migrants would be increasingly perceived (and categorised) as citizens. Like all other citizens, migrants would have the same rights and duties to participate within Dutch society. Such a perspective on citizenship introduced the logic of equity rather than the logic of minorities, thereby contributing to the rise of universalism and the fall of multiculturalism. ${ }^{126}$ Paying attention to specific characteristics of minorities - for instance, their ethnic or cultural status - now receded more to the background.

A concrete illustration of the logic of equity versus the logic of minorities concerns one of the central issues in this period: the interpretation of data on immigrant unemployment. As detected from the logic of minorities, immigrant unemployment is explained in relation to specific traits of minorities. This concerns their ethnic or cultural status and the problems of discrimination that this could raise, or their social position and the problems associated with specific sectors in which migrants are employed (low-wage, low-education, labour-intensive industries). From this perspective, therefore, immigrant unemployment requires a specific approach to the specific situation of minorities. In contrast, as perceived from the logic of equity, immigrant unemployment is compared with levels of unemployment among natives. Higher unemployment among migrants would mean that migrants perform weaker on the labour market because of specific deficiencies in comparison to natives. Consequently, an approach would have to be developed to counter these deficiencies, rather than one that takes these deficiencies into account as is the case with the logic of minorities. 
The logic of equity was clearly manifest in the focus on citizenship that emerged in integration policy, as well as in the functional decentralisation of such policy to various sector departments. This illustrated how the integration policy was more general than the specific minorities policy. Furthermore, an important facet of this logic of equity was its emphasis on breaking taboos concerning immigrant integration. These alleged taboos would have prevented migrants from being treated as equals with Dutch natives. In the early 1990s, the politicisation triggered by Lubbers - who was 'losing his patience with minorities' (Het Parool 26 March 1990) and Bolkestein - who stressed the importance of universal liberal principles - broke these taboos. A new realist discourse emerged, championing openness in the debate about immigrant integration, including negative facets such as criminality and reliance on welfare state facilities. This shift towards the logic of equity in part reflected a more general trend in government policies. The politics of welfare state retrenchment involved a broader trend from 'soft-compensatory' to 'achievement-based' policy measures.

\section{Minorities research: Scientific habitus at stake}

Also in the field of scientific research, the enlightenment configuration posed a challenge to the established logic of minorities. Although it did not alter the structure of the field of scientific research to the same extent as the policy field (the minorities paradigm was not dissolved, but structurally challenged by a rival citizenship paradigm), it, too, contributed to a different logic of framing immigrant integration in this field. The enlightenment configuration challenged the established way of studying immigrant integration, which was highly influenced by the anthropological tradition. This involved specific research methods (field research) and a specific ethos (engagement with minorities). The enlightenment research-policy nexus created an opportunity for developing an alternative research perspective, beyond the established field structure and ACOM's role in that respect.

WRR was notably central in this respect. Because of its structural position beyond the scope of the immigrant integration research field and its central position in relation to government, WRR provided an effective venue for experts such as Albeda and Entzinger who advocated an alternative perspective on immigrant integration. Furthermore, the interdisciplinary composition of WRR played an important role in developing a broader scientific perspective on immigrant integration. This was illustrated by the involvement of, besides Entzinger, experts from other specialisations.

This structural position and multidisciplinary composition enabled WRR to punctuate the dominant logic of minorities in the field of immigrant integration research. It was not institutionally involved in the research of minorities alone but, as we have seen, carried a broader agenda on welfare state 
reform in this period. Furthermore, because of its multidisciplinary composition, its perspective on immigrant integration was adopted from disciplines, such as economics and law, that are generally colour-blind - or at least blind to the relevance of ethnic or cultural factors. In fact, involving a council member of a legal background majorly influenced the making of the 1989 WRR report and in developing a principle of 'substantive neutrality', indicating that the government should observe constraint in cultural policies.

Thus, in both fields, the enlightenment configuration contributed to a more generalist focus on minorities, shifting the specificity of the status of migrants to the background. By connecting immigrant integration to broader concerns about welfare state reform and citizenship, the logic of equity replaced the logic of minorities in policy development. In research, too, a logic of equity emerged, a consequence of the WRR's multidisciplinary, multi-sector scope.

\subsubsection{Enlightenment and frame reflection?}

Enlightenment contributed specifically to the fall of multiculturalism and the rise of universalism in both research and policy in the Netherlands in the early 1990s. However, did it also contribute to critical dialogues between research and policy on the new universalist model of integration? In other words, did these research policy dialogues involve openness, empathy, critical reflection, pragmatism and trust?

\section{Opportunities for frame reflection}

In specific respects, the enlightenment boundary configuration did contribute to frame reflection. First, it had an important effect on the openness of debate in terms of putting immigrant integration back on the policy agenda. The relative insulation of this policy domain, supported by scientific involvement, had hampered critical reflection of the underlying problem frame. Expanding this issue and drawing more actors into the debate on immigrant integration promoted the first condition for frame reflection openness. Immediately following the 1989 WRR report, the debate became more open in terms of public discussions, but also in terms of debate within the scientific field. Following the national minorities debate, the discussion became more open in the political domain as well; this marked the first time the domain had become politicised.

Furthermore, the enlightenment configuration put an alternative problem frame on the agenda, a frame of the universalist type, which conflicted in fundamental respects with the dominant multiculturalist frame. This punctuated the monopoly of the multiculturalist frame. By developing an alternative frame, the debate on immigrant integration - in research as well as policy - was lifted to the fundamental level of how the problem was framed. It 
also broke with some of the prevailing taboos on alternative frames that had hindered frame reflection. For a long time the multiculturalist frame was imperative, as other frames were often criticised for stigmatisation and minorisation. Now the formulation of an alternative frame - one not emphasising the risks of stigmatising minorities but rather, approaching migrants as equals - created opportunities for free reflection on different frames.

Thirdly, and highly related to the aforementioned point on formulating an alternative frame, the enlightenment boundary configuration contributed to critical reflection. The rise of an alternative frame on the agenda provoked frame conflict, thereby making both frames more explicit. The emergence of frame conflict within both the policy and the scientific field forced involved actors to reflect upon their own frames, instead of taking them for granted. The amassing sense of urgency following the 1989 WRR report and the national minorities debate contributed even further to this structural condition for critical reflection.

\section{Constraints on frame reflection}

Although the enlightenment boundary configuration did provide some of the structural conditions required for frame reflection, it did not contribute to frame reflection in some other respects. This concerned, primarily, the sense of pragmatism that is required for gradually adapting one's frame in response to critical reflection. Instead, such pragmatism was obstructed by the open conflict that emerged in policy as well as research. On one hand, WRR, supported by the Minister of Home Affairs, stressed the fundamental difference between the new frame that they advocated and the prevailing multiculturalist frame, rather than advocating pragmatic adaptation between both frames. For instance, the aim was confronting the status quo, rather than reflecting on it or providing a pragmatic adaptation of frames. This was achieved by, among other things, deliberately defining immigrant integration in terms of allochthonous, integration and citizenship, so as to mark the difference with the prevailing minorities policy. On the other hand, criticism by actors involved in the status quo hardly indicated a sphere of pragmatism. This criticism was often moralistic with, for example, accusations that the WRR report would cause stigmatisation. It was also personally directed towards the Prime Minister after his statements in the 1990s and, later, to Bolkestein. The nature of this censure often contributed to confrontation instead of reflection. This is also true for the personal attacks of character experienced by Entzinger and his involvement in the 1989 WRR report, aimed to discredit his 'intellectual leadership' because of his different way of perceiving and approaching the issue of immigrant integration.

Secondly, the enlightenment configuration did not provide the trust among involved actors that is required for frame reflection. It evolved in a context of progressive distrust in this policy domain, between actors 
supporting the status quo and actors that advocated policy change. In fact, the enlightenment configuration seems to have been a deliberate design for breaking the monopoly of the status quo. The official demand for scientific advice from the authoritative WRR created an alternative venue for policy change. In the preceding analysis, we saw that this process went largely beyond the actors involved in the policy status quo, such as ACOM and the Minorities Policy Directorate.

Whereas the enlightenment boundary configuration set some conditions for frame reflection, it also obstructed reflection in other respects. Putting immigrant integration back on the public and political agendas, developing an alternative frame and forcing a critical revaluation of the prevailing policy frame encouraged reflection on problem framing. However, this occurred in a confrontational, distrustful context rather than one of pragmatism or, much less, trust. In fact, the enlightenment configuration created a venue for problem framing beyond the scope of actors engaged in that of the prevailing status quo, sometimes leading to sharp conflicts of a moral and personal nature.

\subsection{Conclusions}

Contrary to the dominant image in migration literature that the Dutch multicultural model persisted long after the 1980s, a new model of integration had already emerged (at least in formal policy discourse and in research) during the late 1980s and early 1990s. While social researchers were crucial in constituting the multicultural model of the 1980s, now they played a major role in putting a new frame of immigrant integration on the agenda. However, the precise configuration of research-policy relations majorly differed. The technocratic symbiosis of the 1980s included the close involvement of researchers in constructing the structural monopoly that sustained the multicultural model. This continued to insulate the domain from broader societal developments throughout the 1980s. Now, a more enlightenment type of research-policy nexus created a legitimate way to punctuate this monopoly, linking immigrant integration to broader social and political developments, such as the politics of welfare state retrenchment and developing stricter 'universalist' policies. In the field of research, this reconfiguration of research-policy relations also allowed for the rise of an integration paradigm that challenged the dominant minorities paradigm.

This episode in research-policy dialogues on migration and integration clearly reveals how the configuration of these dialogues is itself very much an object of strategic interaction. For a long time, the structural equilibrium of the 1980s had been maintained through effective processes of negative feedback (including depoliticisation, taboos, consensus on specific measures in times of economic recession). Yet, WRR's authority, the framing 
of its 1989 report 'Immigrant policy' and its perspective on welfare state reform, proved crucial in setting the agenda of a new problem frame. Furthermore, for a number of politicians who were unhappy with the lack of reform in integration, WRR furnished a venue to put a more universalist approach to integration on the agenda, in a way that would be considered legitimate and authoritative.

Thus, the enlightenment nexus was a clear product of strategic design, made in an effort to punctuate the structural equilibrium of multiculturalism that emerged in the 1980s. Although this enlightenment, in accordance with the enlightenment ideal type, did not immediately lead to policy change, it did alter political and social discourse. It generated policy feedback and it also invited negative policy feedback, particularly from those research and policy actors involved in the technocratic symbiosis of the 1980s (such as ACOM). When policy developments gained more momentum after the 1991-1992 national minorities debate, many elements of the 1989 WRR report were revived and adopted in the new integration policy. Even in this period, experts who had been involved with the WRR reports continued to play a central role in ensuring that policy change was effectuated. However, after policy reframing took place, more political primacy emerged in research-policy relations. In particular, the Department of Home Affairs became a more prominent participant in the coordination of instrumental research that was provided by SCP. This suggests that following the establishment of the universalist frame in policymaking in the early 1990 s, a more bureaucratic boundary configuration emerged.

The enlightenment nexus ended the relative insulation of this policy field from broader developments, connecting immigrant integration to wider concerns about the welfare state. Thus, it brought an end to the logic of minorities in problem framing that had sustained the multicultural model. Rather than focusing on the (cultural, ethnic) specificities of minorities, it contributed to a reframing of immigrants as equals - citizens - involving a multidisciplinary perspective that was blind to group-specific factors via the logic of equity.

The enlightenment configuration was designed by political actors and WRR to punctuate the structure-induced equilibrium in minorities policy and research. It also had the unintended effect of contributing to frame reflection, leading to an open scientific and political debate about immigrant integration (punctuating the structure-induced equilibrium of the 1980s), and providing a legitimate, acceptable venue for an alternative frame (universalism). Furthermore, it forced critical reflection on alternative frames through freely confronting frames in public, political and scientific debates. However, the enlightenment configuration also constrained frame reflection because it aimed to confront established minorities policy and research rather than promote reflection about multiculturalist or universalist frames in a sphere of pragmatism and trust. 



\section{The engineering of the assimilationist turn (2000-2004)}

Around the turn of the millennium, many Western European countries experienced what has been described as 'an assimilationist turn' in their immigrant integration policies (Joppke \& Morawska 2003). The Netherlands is, arguably, the country where such an assimilationist turn manifested itself in a significant manner, at least in national policy discourse. In 'the long year of 2002', as it was called (see section 6.1.1), immigrant integration was to become the central issue in one of the most dramatic episodes in Dutch post-war political history, involving the rise and subsequent murder of the populist politician Fortuyn. At the same time, the multicultural model, for which the Netherlands had once been so well known, was now broadly discarded as 'a failure' and turned into a prominent counterdiscourse against which new policy developments were juxtaposed.

The research-policy nexus would, more than ever before, itself become the object of intense controversy in the sharp politicisation of immigrant integration in this period. A technocratic model of government was now denounced together with the multiculturalist model with which it was often associated. Because of their intense involvement with policy developments in previous periods, the credibility of immigrant integration researchers was publicly put on the line. This episode will show how difficult it is to organise reflective research-policy dialogues on intractable controversies such as immigrant integration, and how much the construction of these dialogues is entwined with the construction of social problems like immigrant integration.

Controversies about the research-policy nexus make this episode even more interesting in terms of studying the co-evolution of immigrant integration research and policy in the Netherlands. For instance, why did a third report go ignored from the, by now, highly authoritative WRR, 'The Netherlands as immigration society' (2001)? This does not mean that research played no role in ongoing developments in this period. In fact, the SCP would venture beyond its capacity as a data provider to become an open advocate for an assimilationist approach. In addition, a parliamentary investigative committee would commission an extensive survey of available migration and integration literature in order to establish why policy had, thus far, only been 'partly successful'. However, internationalisation of the research field and the growing involvement of European institutes in 
the co-optation of immigrant integration researchers inspired trends to frame migration and integration as transnational issues. This development ran, of course, directly against emergent national framing in the field of policymaking.

\subsection{The turn towards assimilationism and transnationalism}

In chapter 4 we saw that, at least in formal policy discourse, a frameshift occurred from universalism towards assimilationism. In this respect, the Dutch case seems to resemble other countries that experienced an assimilationist turn in this period. Also in the field of research, assimilationist knowledge claims surfaced. However, as previously observed, the trend in this field is better seen in terms of the growing fragmentation of knowledge claims. In fact, besides multiculturalism, universalism and the new assimilationism, there was a clear rise of transnationalism in the field of immigrant integration research. In particular, there was a widening divide between research and policy in terms of their transnationalist and assimilationist framing of immigrant integration. In what situational setting did this split emerge, and which main research and policy actors were involved in research-policy dialogues in this period?

\subsubsection{Context: Internationalisation and national identity}

Several contextual developments during the 1990s and around 2000 put stress on the structural equilibrium that had evolved around the universalist policy approach earlier in the decade. One development that induced actors to reframe immigrant integration was the ongoing process of internationalisation or, as others called it, globalisation, which had a mounting effect on various institutions, including government and science. The establishment of the European Union during the 1990s and its increasing influence on various facets of national policies were important indications. In addition, the national welfare state, which was a central point of reference for Dutch integration policy in this period, was progressively affected by developments at a European level. Scientific research also became more internationalised. Sometimes aided by the development of European institutions (such as EU research funding), more and more international research networks evolved.

Internationalisation also affected immigrant integration. Free movement of people within the EU and better means of global transportation and communication surged migration flows to EU countries. In response, EU governments constantly undertook joint activities to limit migration across EU borders, contributing to the rise of a so-called 'Fortress Europe' (Geddes 2003). Besides the development of a joint European immigration 
policy, EU institutes became more active in the domain of immigrant integration (ibid.), especially concerning anti-discrimination regulations (Guiraudon 2006). This also influenced the development of EU research networks in the field. Whereas immigrant integration research had, thus far, been confined mainly to the context of nation-states (Favell 2005; Lavenex 2005), now migration scholars became increasingly involved in networks beyond their nation-states and, according to some, even cooperated in EU government networks (Geddes 2005).

Ongoing immigration, especially family and asylum migration, had ever more visible effects in society. 'Old' migrant groups grew due to demographic effects such as high fertility rates and ongoing family migration. In the Netherlands in 2000, 2.8 million immigrants (comprising 17.5 per cent of the Dutch population) had either been born outside the Netherlands, or had at least one parent born outside the Netherlands. ${ }^{1}$ In the major cities of Amsterdam and Rotterdam, immigrants comprised, respectively, 31.1 per cent and 30.5 per cent of the total population. ${ }^{2}$ Furthermore, asylum migration contributed to the rise of new migrant groups, such as those from the former Yugoslavia, Iraq, Iran, Afghanistan and Somalia. The communication and transportation means that facilitated migration flows on a global level also facilitated the orientation of migrants towards their home countries, for instance, through satellite television and the internet. Cultural diversity became further institutionalised in Dutch society, as illustrated by the establishment of numerous mosques, Islamic schools and even Islamic universities.

WRR was one of the first actors to reconsider the prevailing perspective on immigrant integration in the context of these developments. In its third report on immigrant integration (WRR 2001b), WRR claimed that the Netherlands had become an immigration society and that immigration and integration policies, as well as the Dutch social state itself, had to adapt to this development.

There was reluctance in Dutch society to adapt to this prospect of an immigration society. In fact, there were indications during this era that the integration process was advancing rapidly in the social-economic areas of labour, education and housing, all key components of the integration policy. Unemployment levels among immigrants had declined significantly since the early 1990s, from over 30 per cent among Turks and Moroccans and over 20 per cent for Surinamese and Antilleans in 1994, to under 10 per cent for all these groups in 2000 and 2002. The government's aim to reduce unemployment among immigrants by 50 per cent had been achieved by 2001 (SCP 2001: 14). Nonetheless, the level of immigrant unemployment remained more than double that of the average native population. Labour market participation among minorities also grew significantly, although, again, it was still less than among natives. The position of immigrants in education was ameliorated as well, despite persistent differences 
between the groups (notably, the weaker position of Turks and Moroccans) and their sustained gap in education compared with natives.

A series of political and societal developments after the turn of the millennium changed the direction of policy developments. A second national minorities debate emerged in 2000, the so-called Scheffer debate, which focused attention on an alleged 'multicultural tragedy'. Secondly, a series of events widely discussed in Dutch media and politics drew further attention to an alleged 'clash of civilisations' (Snel \& Scholten 2005). This included incidents with acts of violence that involved immigrants, as well as morally questionable events that focused attention on the dilemmas of cultural and religious diversity, including imams making radical statements about homosexuals and refusing to shake hands with the female Minister for Integration (ibid.). The international developments surrounding the terrorist attacks of $9 / 11$ also led to fierce anti-Muslim responses in the Netherlands, as was the case in other European countries (Fennema 2002).

Especially groundbreaking was 'the long year of 2002' when the populist politician Fortuyn brought immigrant integration to the centre of public and political attention. Fortuyn was the political leader of the newly founded party Liveable Netherlands (Leefbaar Nederland) in 2001 and led the local branch Liveable Rotterdam in the local elections of 2002 . He rapidly became the subject of controversy because of his tough media statements, calling for 'zero-immigration' because the Netherlands was 'full', calling for a 'cold war against Islam' and dismissing Islam as 'an idiotic culture'. ${ }^{3}$ Following these statements, Fortuyn was fired by his own party as their political leader and founded his own: the Pim Fortuyn Party (LPF). During campaigning for the 2002 parliamentary elections, an animal-rights activist assassinated Fortuyn on the very day the polls showed his party would come first. Following these elections, the LPF eventually became the second-largest party and was included in a centre-right government coalition with the Liberals and the Christian Democrats. The LPF was to be included in a centre-right government coalition with the Liberals and the Christian Democrats.

The 'long year of 2002' had two direct political effects. Firstly, it led to the initiation, in the year following the elections, of a parliamentary investigative committee on the integration policy. The aim of this committee was to examine why the integration policy had been so limited in its success and also to provide building blocks for new policy initiatives. ${ }^{4}$ Partly based on an extensive study of scientific sources by the VWJ, this committee eventually concluded that the integration policy had actually been relatively successful. This declaration provoked fierce controversy in public and political debates.

A stronger political leadership emerged in migration and integration policy during 2002. The responsibility for immigrant integration and immigration policy shifted towards the Department of Justice. LPF member 
Hilbrand Nawijn was Minister of Immigration and Integration for a relatively short time. When this brief coalition fell in 2002 and a new centreright coalition was formed in 2003 without the LPF, immigration and integration once again found strong political leadership under Minister Verdonk from the Liberal Party. Verdonk was central to the development of several memoranda in which the contours of an integration policy 'new style' were elaborated. Although the effects of this new policy discourse on policy practices seems to have been limited (Poppelaars \& Scholten 2008), the policy documents from this period reveal a change in how immigrant integration was framed in government policies, from universalism towards a more assimilationist policy frame.

In both politics and research, more attention was being devoted to the social-cultural dimension of immigrant integration. In contrast to the transnationalist discourse of WRR, SCP attributed attention to the social-cultural integration of migrants in Dutch society. SCP was also more explicitly involved in advocating a more assimilationist approach in this period.

This context analysis shows how several actors were involved in research-policy relations. In the field of research, this once again involved WRR, reflecting the internationalisation of research in this domain. This time around, however, it seems to have been less influential in terms of policy framing. SCP also continued to be involved at this time. Furthermore, the parliamentary investigative committee and the scientific study it commissioned from the VWJ seem to have been the result of a combined effort by research and policy actors. Finally, in the field of policymaking, the political leadership of the Department of Justice, the new coordinating department for the integration policy 'new style', clearly played a prominent role in policy changes in this period.

\subsubsection{WRR: 'The Netherlands as an immigration society'}

In 2001, WRR published a third report on immigrant integration entitled 'The Netherlands as an immigration society'. It contrasted with earlier ones by placing immigrant integration more in the context of immigration policy and the Dutch rule of law, whereas previous reports had focused more narrowly on immigrant integration (De Jong 2002). WRR argued that inadequate integration of migrants into citizens could create tensions in Dutch society. It also stated that ongoing immigration could negatively affect the absorption capacity of society (WRR 2001b: 25).

While the first WRR report (1979) suggested immigrants would not return to their home countries and had become permanent minorities and the second WRR report (1989) claimed immigration as such had become a permanent phenomenon and that the Netherlands had become a de facto country of immigration, this third one argued that 'the permanency, diversity and unpredictability of migration' and the 'sharp increase of diversity 
since the 1980s [...] also have consequences for the (organisation of) Dutch society $[\ldots]$ and the capacity of Dutch society and its institutions to cope with (cultural) differences and take benefit of the positive contribution of immigrants' (ibid.: 19). It adds that, although the second WRR report's statement that the Netherlands had become a country of immigration was now widely recognised, 'the translation of this fact into conduct and policy takes effort' and that 'basically, Dutch government policy is insufficiently directed at the immigration society' (ibid.; author's emphasis). Such an immigration society would demand 'a capacity from Dutch society and its institutions to cope with [cultural] differences and take profit from the contribution of migrants' (ibid.).

WRR did not adopt a new way of classifying immigrants, as it had done in the two earlier versions. It used the term 'migrant' or 'immigrant', but also 'allochthonous' and 'newcomers'. An important argument for using the comparatively neutral depiction of 'migrants' was that referring to second- or third-generation migrants as 'allochthonous' could have a labelling effect that supported the intergenerational character of being an immigrant (ibid.: 32). The report also speaks more of 'participation' than of 'integration'. The former term was used referring to the position of migrants, whereas the latter connoted mutual interdependence within society. The general use of the term 'integration' referring to the incorporation of migrants into society was not adopted in this report.

In contrast to the two earlier reports and despite reference to the Netherlands' fundamental transformation into a so-called immigration society, WRR does not explicitly call for a fundamental policy shift. In fact, it takes sides in the controversy over policy success or failure by claiming that government should resist 'the pressure to [...] formulate new policies, instead of striving to ameliorate the implementation of current policies' (ibid.: 180). Despite the often 'strongly ideological character of policies concerning immigration and integration', WRR calls for a more instrumental and procedural policy approach, one which focuses primarily on those areas that best lend themselves to government intervention, such as language proficiency, labour and education.

WRR dissociated itself from the emerging assimilationist policy discourse. In important respects, it built on the universalist frame of the 1990s, for instance, in its emphasis on the principles that migrants have a personal responsibility to participate (citizenship) and that immigrant participation requires accessibility to institutions. The report formulates principles of 'encounter' and 'confrontation'. This concerns the promotion of interaction within society, avoiding the formation of segmented societies that threaten the integration of society at large, without necessarily involving assimilation. The task of government in this respect would not be substantial but rather instrumental, creating the conditions for interaction. 
These principles underline that the aim of assimilation, in the sense of the absorption into Dutch culture, is neither desirable nor necessary in a highly diversified immigration society. However, for the ongoing existence of the social-democratic system in the Netherlands, it is necessary to lay down certain requirements for immigrants and the host society. The requirements imposed on immigrants are aimed at their participation in Dutch society and those for the host society at the accessibility of the institutions of the social state and adjustment to the cultural diversity of the population. (ibid.: 25)

However, the report also adds a cultural dimension to this frame that reflected more of a transnationalist than an assimilationist frame. Whereas both universalism and multiculturalism contain a clear national perspective on immigrant integration (the Dutch multicultural society, the Dutch functionalist state), WRR now viewed immigrant integration through the lens of internationalisation. There are numerous references to the fact that 'the world has become smaller', that cultures have become more dynamic, that individuals develop more and more hybrid or multiple identities and that migration has led to the formation of transnational communities that keep their one foot in the country of origin and one in the destination. Without explicitly taking this transnationalist perspective as normative, internationalisation became accepted as a way in which policy should be adapted. To do this, the report set a more positive tone on migration as an element of internationalisation. In fact, it put forward 'an approach that would maximise the benefits of immigration, both for Dutch society and for the newcomers' (ibid.: 9). It explicitly denounced the 'programmatic and normative tone' of the debate on the multicultural society (ibid.: 22).

\subsubsection{Social and Cultural Planning Office (SCP)}

In the early $1990 \mathrm{~s}, \mathrm{SCP}$ gained prominence for providing social-economic data on immigrants. It had acquired an important role in the structureinduced equilibrium of the universalist approach with the provision of instrumental data in its regular minorities reports. During the 1990s, SCP's minorities reports remained an important tool in the interdepartmental coordination of the integration policy. They occasionally contributed to important policy corrections, for instance, in 1996's housing policies (VWJ 2004: 138). More generally, because of its involvement in other domains and high media profile, SCP became more established in this period, with authority in the political field and the field of scientific research.

At the end of the 1990s, SCP adopted a more assimilationist problem framing in response to, among other things, indications of decline in the acceptance of minorities and cultural diversity in Dutch society. Firstly, in 
its 'Social and cultural report' from 1998, SCP explicitly dissociated itself from the idea that the Netherlands would have, or should become, a multicultural society. ${ }^{5}$ SCP (1998: 8) claimed that 'although Dutch society does increasingly contain people of different cultural origins, the secular, universalist, individualist, in short Western culture experiences barely any competition from other streams'. Moreover, cultural goods, such as norms and values, would have to be clearly distinguished from social goods such as labour and income, which can be discussed in terms of individual rights and duties. According to SCP, cultural goods cannot be differentiated for various individuals and groups. Instead, they represent an 'intrinsic hierarchy', ruling out the possibility of 'essentialistic pluralism' as this would lead to 'as many public spheres as cultural groups' (ibid.: 267). In this context, the importance of 'cultural assimilation [would have been] underestimated and ignored by government, agents and social scientists' (ibid.: 271). SCP had already made similar statements in its 'Social and cultural report' from 1986, but refrained from repeating these observations ever since becoming more structurally involved in the domain in the early 1990s.

In 1998, the newly appointed director of SCP, Schnabel, explicitly argued for 'adaptation and assimilation', claiming that the multicultural society was 'an illusion'. He did so in a lecture for the Institute for Multicultural Expertise (FORUM), and in an essay (Schnabel 1998) and a newspaper article in 2000. ${ }^{6}$ He based his argument on 1998's 'Social and cultural report'. 7 According to Schnabel (ibid.: 8), becoming a multicultural society was 'neither a realistic nor a desirable option'. He saw socialcultural adaptation as an important means for achieving integration or, as he called it, 'assimilation' (ibid.: 25). Concerning central cultural areas such as the Constitution and civil rights, Schnabel argued that there was no other option but complete assimilation. In other public areas, such as school and work, some degree of integration or assimilation could be expected, whereas in the more private cultural spheres, such as home, family and church, there would be more space for diversity or even segregation. ${ }^{8}$

SCP's minorities report also paid more systematic attention to 'socialcultural integration'. For the first time it included an empirical analysis of data on social-cultural integration in a preparatory study it made for the 2001 WRR report on immigrant integration (Dagevos 2001). In the context of rising concerns about social-cultural diversity and ethnic cleavages in society after the turn of the millennium, SCP decided, together with the coordinating Department of Justice, to include social-cultural integration in its minorities report for the first time in 2003 (SCP 2002: 134, 2003: 9). It defined social-cultural integration in terms of the modernisation of specific norms and values, referring to 'a centuries-long modernisation process of western culture [...] which has advanced less in many other parts of the world' (SCP 2003: 9). According to SCP: 
Social-cultural integration stands [...] for the extent to which ethnic minorities share a number of the basic values and norms of Dutch society and the extent to which they have contacts with natives. A modern western world view was taken as a point of reference for the choice of these norms and values, which is characterised by the idea of individual human development, social equality (also between men and women), secularism and a democratic regulation of power (ibid.: 9).

In the 2003 minorities report, SCP provides a differentiated image of the process of social-cultural integration. In terms of modern cultural attitudes, there were signs of cultural assimilation, especially in second- and thirdgeneration migrants of specific groups (SCP 2003: 427). However, in terms of religious attitudes and social contacts, it appears that there was a growing divergence between natives and immigrant groups (ibid.). Turks and Moroccans had an especially pronounced orientation towards their own cultural group, whereas Antilleans and Surinamese were more assimilated into Dutch society (ibid.: 430). As for social-economic participation, SCP signalled that, despite the significant progress made in the previous decade, persistent problems remained. They involved, among other indicators, a persistent relative deprivation in education and labour, the latter of which was deteriorating due to overall economic decline.

SCP would continue to draw attention to the social-cultural dimension of integration. Following the 'long year of 2002' when the populist LPF was eventually incorporated into government, the Minister of Immigration and Integration asked SCP to coordinate a research project on Islam in Dutch society. This eventually led to 2004's 'Muslims in the Netherlands'. 9 This report concluded that although secularisation did occur among Muslims in terms of religious practices, the group's religious identity underwent a religious revitalisation. SCP focused on social-cultural integration in other reports as well as - and often in relation to - socialstructural integration. ${ }^{10}$ Although the regular minorities reports were discontinued after 2003, SCP continued to issue documents on this matter, including the 'Annual integration report' (2005) that was published together with the Statistics Office (Centraal Bureau voor de Statistiek, CBS) and the Scientific Research and Documentation Centre of the Department of Justice (Wetenschappelijk Onderzoek-en Documentatiecentrum, WODC).

\subsubsection{The Blok Committee and the Verwey-Jonker Institute}

Following the controversial parliamentary elections of 2002, Parliament took the initiative in establishing an investigative committee to examine the integration policy. Dutch Parliament has a constitutional right to establish investigative parliamentary committees or parliamentary inquiries not 
only to look into social problems but also to evaluate policies. They form an important means through which Parliament can alleviate its relatively asymmetrical access to information compared to government, which has a great number of advisory bodies at its disposal, including WRR and SCP (Halffman \& Hoppe 2006). ${ }^{11}$ Although this right had existed for a long time, it only recently came to be used more frequently by Parliament, particularly for the investigation of policy fiascos (Andeweg \& Irwin 2005: 144). This development was also institutionalised with the establishment of a Research and Verification Office (Onderzoek en Verificatie Bureau, OVB) in 2002, which provided administrative support to parliamentary inquiries and investigative committees. In this case, Parliament established an investigative committee, which has fewer formal powers than a parliamentary inquiry, but can have a similar policy impact.

The parliamentary motion that demanded the establishment of an investigative committee was submitted by the Socialist Party, who advocated a more assimilationist policies (Fermin 1997: 112). Previous attempts by this party to call for a parliamentary investigative committee had been fruitless. ${ }^{12}$ Now, though, Parliament accepted a motion concluding that integration was 'insufficiently successful' and called for an evaluation of the causes for failure.

Concluding that the integration policy has thus far been insufficiently successful, observing that it would be desirable to evaluate what may have been the cause of this, observing that such research could provide the building blocks for the formulation of a new integration policy, Parliament decides to establish a parliamentary research committee on the integration policy. ${ }^{13}$

In fact, there was already an evaluation of policy failure present in this stage of the research problem formulation. However, members of the LPF voted against this motion; as for them, the conclusion that the integration policy had failed was already so clear that they believed a special research committee was unnecessary. Perhaps more importantly, the LPF did not want to gain any new 'building blocks' for an integration policy; rather, it wanted to abolish the integration policy altogether and incorporate policies towards immigrants into general government policy. ${ }^{14}$

Whereas the initial idea for establishing the investigative committee was based on an assimilationist evaluation of the integration policy - as insufficiently successful or 'failed' in the social-cultural terrain - eventually its official aim was reformulated in a more open way, by a parliamentary working group that made preparations for establishing the committee. The final assignment given to the committee also contained no specific focus on social-cultural issues, something that would have been expected in an assimilationist framing of the investigative committee's aim. Instead, the 
goal of the committee was 'to enable the Second Chamber of Parliament to evaluate the integration policy of the Dutch government over the past 30 years, to evaluate the aimed effects and factual results of this policy and to evaluate the coherence of policy in various policy sectors'. ${ }^{15}$ Also, 'the research should provide building-blocks for the integration policy to come'. The various policy domains considered relevant included income and labour, education, housing and recreation and sports.

The report by the investigative committee, named the 'Blok Committee' after its chairman, Stef Blok, was for a large part based on a study by the VWJ, although the reports of this committee and the institute also differed in important respects. The VWJ was asked to undertake an evaluative study of the goals and results of the integration policy over the past decades in specific areas and to determine to what extent policy had been consistent and coherent. ${ }^{16}$ Moreover, it was asked to determine the extent to which the integration policy 'could be qualified as successful'.

This last question, in particular, related to the framing of immigrant integration. The approach adopted by the VWJ was manifest in its assumption that 'success in one domain can be of higher importance than that in others' (VWJ 2004: 196). The 'success in the domain of education appears [to be] the key for the further success of the integration process', meaning that the results in this area are of extra importance. It was this assumption that eventually led the institute to the conclusion that the integration policy had been 'relatively successful' (ibid.). Furthermore, it concluded that 'part of the intended goals have been realised [...] especially in the domain of education', as well as in the domain of housing, whereas the goals in the domain of income and labour 'have been less achieved' (ibid.).

This emphasis on education and labour as corner stones of integration reveals the VWJ's universalist framing. Thus, it founded its conclusion about the relative success of the integration policy on achievements in the social-economic, rather than the social-cultural, domain. The institute also put its conclusions in greater context, providing an incentive for critical reflection about this problem frame. The lack of complete success was due to inconsistencies between policies, it argued. Another conclusion was that 'especially in the social-cultural domain there is a gap between formulated objectives and results that have been attained thus far' (VWJ 2004: 197). There was warning against an 'anachronistic evaluation' of policy results accumulated from the past but based on policy objectives set out only recently: 'results are lagging mainly in areas where only recently new and sharper goals have been formulated' (ibid.). Here, the VWJ appears to be using the fact that this had been the dominant frame in the previous decade as a way of legitimising its choice for a universalist approach, thereby dissociating itself from the new assimilationist frame to emerge in the political discourse during that time. It did, however, raise the question of whether, 
given the 'recent political turbulence, the goals have always been formulated sufficiently broad in the past' (ibid.: 198).

The Blok Committee formulated a definition of 'integration' to use for determining whether the integration was successful or not. This definition was universalist in character, emphasising social-economic participation, equality in legal terms and the 'two-sidedness' of integration. The definition also contained some elements that could lead either to a more assimilationist framing (respect for common values, norms and patterns of behaviour) or even a multiculturalist framing (integration as a person or a group). However, the committee failed to elaborate the definition to such an extent that the relative importance of these various indicators of integration could be established. The committee adopted the following definition of successful integration:

A person or a group is integrated in Dutch society when they have an equal legal position, equal social-economic participation, knowledge of the Dutch language and when common values, norms and patterns of behaviour are being respected. Integration is a two-sided process. On the one hand, newcomers are expected to be willing to integrate; on the other hand, Dutch society must make this integration possible. (Blok 2004a: 105)

Based on this definition, the Blok Committee concluded that 'the integration of many immigrants has been a total or partial success and [...] this is quite an achievement for the involved immigrant citizens as well as for the host society' (Blok 2004a: 105). The way in which the committee substantiated this conclusion revealed a more universalist framing than its definition of integration would suggest. Its belief in the success of the integration process was founded especially on the progress made in education, labour, housing and women's emancipation (ibid.: 522). These domains mainly concern the individual participation of migrants, regardless of gender, rather than group emancipation (as in multiculturalism) or social-cultural integration (as in assimilationism).

An important difference from the VWJ was that the Blok Committee did not conclude that the integration policy had been successful but rather, that the integration process had been successful. The committee observed that 'causal relations with the general integration policy are difficult to prove [...]' (ibid.: 522). The success of the integration process would have been especially affected by general developments in society and by the efforts of those migrants involved. In this respect, too, the Blok Committee seems to have followed a universalist frame in which the government had only a supporting role in the integration process.

The Blok Committee and the VWJ produced what could be expected from an evaluative study of the integration policy - evaluating policy 
effects based on given policy goals. Adopted was the universalist problem frame of 1990s integration policy and, based on this frame, both actors came to the conclusion that integration had been rather successful. However, within the context of ongoing policy debates after 2000, they also continued to propagate a universalist frame. They pinpointed education and labour as the key sectors of integration. The Blok Committee even concluded that, given the progress achieved in those sectors that were considered vital from a universalist frame of immigrant integration, the integration process - though not so much the integration policy - had been relatively successful.

\subsubsection{Politics and public intellectuals}

Finally, political actors and so-called public intellectuals who were main players in public, political and sometimes scientific debates became more involved in policymaking. They ensured that immigrant integration topped the political agenda during this period (Essed \& Nimako 2006). This would have a direct effect on the policy changes that were made by the two centre-right governments formed in 2002 and 2003.

Two actors particularly had a significant impact on policymaking. First, the public intellectual Paul Scheffer triggered a second national minorities debate in 2000, with a piece published in NRC Handelsblad (29 January 2000) on what he described as the 'multicultural tragedy' taking place in the Netherlands. In this newspaper article, Scheffer adopted a more assimilationist frame, focusing attention on the relation between social-cultural differences and social-economic deprivation. ${ }^{17} \mathrm{He}$ defined immigrant integration as 'the social question of this age' and warned of the development of an 'ethnic underclass' in Dutch society, characterised by 'unemployment, poverty, school drop-outs and criminality'. ${ }^{18}$ He claimed that the 'benign multiculturalism' found in a cultural differences approach was one of the causes of the formation of the ethnic underclass. To achieve better integration, Scheffer called for a 'civilisation offensive [in which] Dutch language, culture and history should be taken more seriously'. Scheffer believed that, "We say too little about our boundaries, cherish no relation with our past and treat language in an ignorant way [...] a society that repudiates itself has nothing to offer to newcomers'. ${ }^{19} \mathrm{He}$ also warned against the growth of ethno-cultural cleavages in society. Scheffer's analysis of the alleged clash of civilisations was an important element in 'The multicultural tragedy' (Prins 2002a; Snel 2003a: 13). The unfolding debate put immigrant integration back on the public and political map in 2000, sparking a lively polemic in the national media (Scholten \& Timmermans 2004). Subsequently, a special parliamentary debate was held among various political party leaders to discuss integration policy 'in general'. ${ }^{20}$ 
The second actor to have an effect on policy was another public intellectual heavily involved in politics. Fortuyn was already known for his conservative columns in the popular Dutch magazine Elsevier and his 1997 book Against the Islamisation of society (Fortuyn 1997). Fortuyn's 2001 entrance into politics marked the beginning of 'the long year of 2002' and one of the most controversial periods in Dutch post-war political history (Andeweg \& Irwin 2005). Fortuyn, even more so than Scheffer, framed immigrant integration in an assimilationist way. He defined migrants in terms of their social-cultural differences in relation to Dutch society. Generally, this involved highlighting the religious status of migrants. He also framed immigrant integration in terms of compensating the 'socialcultural deprivation' of migrants whose upbringing had not been part of Western Judeo-Christian culture. ${ }^{21}$ In order to preserve Dutch culture and identity and to compensate for the social-cultural deprivation of migrants, Fortuyn argued for a more obligatory approach to integration that also involved adaptation to Dutch norms and values.

Although the minorities debate inspired by Scheffer did not immediately lead to policy change, several years later, it would be seen as an important turning point in the development of this domain (Entzinger 2005: 8). The coordinating Minister of Integration and Urban Affairs refuted claims that there would be a 'multicultural tragedy', pointing instead to signals of social-economic progress. Based on the prevailing universalist problem framing, this was evidence that the integration policy was rather successful.

We do not share the feelings of dejection. [...] A warning against an unbalanced idea about the position of minorities in Dutch society is surely in place. There are not only problems, there is also progress. [...] There is no question of a multicultural tragedy. There is no progressive marginalisation of various ethnic groups in the Netherlands. They are (on average) better integrated than in the past. (Van Boxtel 2000: 12-13)

The rise of Fortuyn had a more immediate effect on policy changes. The 2002 elections brought a new government to power with very different ideas on immigrant integration. The populist LPF was a part of the new government coalition and named immigrant integration a top policy priority. The coalition agreement signed by the Liberal Party, the Christian Democratic Party and the LPF emphasised, in particular, the social-cultural dimension of immigrant integration. It highlighted how ethnic differences in combination with discrepancies in education, labour participation and crime rates contribute to 'divergent powers within society and to physical, social and mental cleavages between communities'. ${ }^{22}$ Although it claimed to renounce a politics of assimilation, perhaps in part due to the sensitivity 
of this concept, it argued for 'approaching religious, cultural and ethnic difference on the basis of respect for fundamental values and norms that are characteristic for Dutch society'. ${ }^{23}$

In this centre-right government, LPF member Nawijn became Minister for Immigration and Integration. What is more, responsibility for integration policy was taken away from the Department of Home Affairs, which had been the coordinating department since the late 1970s. The Department of Justice now became coordinator, signalling a more pronounced law-and-order approach to immigrant integration (Entzinger 2003). In 2003, when a new centre-right government was formed, the Department of Justice remained in control for policy coordination in this area, this time with a minister from the Liberal Party.

Rita Verdonk, the new minister responsible for the coordination of the integration policy, produced several policy documents that elaborated a more assimilationist approach to immigrant integration, or the so-called integration policy 'new style'. Details of this policy were also set out in a letter from the minister that accompanied the 2003 minorities report by SCP, which were simultaneously sent to Parliament. Building on observations by SCP on social-cultural integration, the minister observed in this letter that 'in spite of moderate successes in the sphere of education and the labour market, too great a part of the minority population stands at too great [a] distance from Dutch society' affecting 'social and cultural distance as well as economic distance'. ${ }^{24}$ Moreover, the letter explicitly speaks of a turning point in the integration policy, which would involve, in particular, a change from cultivating cultural differences towards 'searching [for] the unity of society in what participants have in common'. ${ }^{25}$

This frameshift towards assimilationism was further codified in the government reply memorandum to the Blok Committee's 2003 report. In this response, the government confirmed the new policy contours that had been elaborated in the ministerial letter. It reiterated its emphasis on social-cultural differences as the incentive for the integration policy 'new style'. Responding to the conclusion of the Blok Committee that integration had been relatively successful, the government stated that it:

endorses the conclusion that especially in the 1990s progress has been made in various domains [...], but that [government] in its own analysis of the actual position [of immigrants] puts emphasis on the social, cultural and economic cleavages that persist. $^{26}$

In this context, the government expressed its disappointment that the Blok Committee had ignored some important facets of immigrant integration, 'in particular the social-cultural aspects of integration and the disproportionate crime rates among some minority groups'. ${ }^{27}$ Moreover, it raised doubts about the 'factual meaning' of the conclusion that integration would have 
been 'a total or partial success'. In fact, the government reframed this conclusion as evidence that integration had been at least partially unsuccessful, or possibly even a complete failure, and that these observations would guide government policy. ${ }^{28}$

This actor analysis clearly shows the multiplicity of frames involved in this period. Recognising this multiplicity creates a very different perspective on the somewhat simplistically described assimilationist turn in Dutch policies. Whereas WRR adopted a transnationalist framing, the parliamentary investigative committee and the VWJ adopted a more universalist frame (in contrast to the parliamentary motion that had led to the establishment of the committee). Moreover, in line with a number of influential political actors and public intellectuals in this period, SCP adopted a progressively explicit assimilationist problem frame.

These actors also selected and interpreted different contextual evidence to support their frames. Whereas universalists selected evidence about the improvement of the position of migrants in crucial social-economic domains, assimilationists chose to select evidence about persistent differences in terms of social-cultural integration or even 'social-cultural deprivation'. While WRR interpreted evidence about ongoing immigration and burgeoning cultural diversity as signs that the Netherlands was becoming an immigration society, actors with assimilationist frames interpreted them instead as indications of a clash of civilisations that demanded more focus on social-cultural adaptation. Furthermore, the attribution of meaning to the focus events that occurred after the turn of the millennium was very important at the time. Actors with assimilationist frames, especially political actors and public intellectuals, played an important role in giving weight to incidents such as the murder of Fortuyn, viewing them as focus events for an alleged 'clash of civilisations'. For instance, even though Fortuyn was murdered by a leftist animal-rights activist, his death acquired the meaning of someone killed for his opinion on multiculturalism and his criticism of Islam, in particular (Essed \& Nimako 2006: 304). The same was true for the murder of the filmmaker Theo Van Gogh in 2004, which also reinforced the idea that discourse on multiculturalism had reached a 'dead end', and that Islam posed a threat to national social cohesion and identity (Hajer \& Uitermark 2007: 7).

\subsection{The research-policy nexus on the line}

As we have seen, controversy over the 'culture' or framing of problems tends to coincide with disaccord concerning the 'structure' of how problems are dealt with by various actors. This is certainly the case in this era, as the division of labour between research and policy became openly contested. Whereas in previous episodes, research made significant 
contributions to policy developments in a technocratic or enlightenment manner, this time the construction of effective research-policy dialogues would prove much more difficult. However, the fact that research-policy boundaries were at stake, as never before in these controversies, makes this an even more compelling episode in which to examine just why the status of research became so contested.

\subsubsection{The field of scientific research}

Fragmentation appearing in the field of immigrant integration research as early as the end of the 1980s would only continue during this period. Within immigrant integration research, a multiplicity of problem frames was manifesting, involving universalist, assimilationist and transnationalist frames. This would also affect relations between different research actors within the field of policymaking, which went through a dramatic assimilationist turn at this time.

\section{WRR: Boundary work beyond the national polity}

In 1997, WRR decided to continue its tradition in the domain of immigrant integration. This led to the report 'The Netherlands as immigration society', published in 2001. Unlike the 1989 report, this document did not respond to a formal advisory request from government. Rather, the initiative for the report was taken as a result of a brainstorming session of the sixth council, which was inaugurated in 1997. The success of earlier reports provided an important motivation for WRR to once more publish a report in this area. The two previous reports had been powerful illustrations of the institutional legitimacy of WRR, and it was considered useful for the council to continue its involvement by producing one report approximately every decade. ${ }^{29}$ A more personal factor involved in this initiative was that council member Pauline Meurs was very interested in this issue. Although immigrant integration was not WRR's academic specialisation, her personal interest was an important motivation for starting this new study. ${ }^{30}$

This time, WRR did not demarcate its role as developer of an alternative design for the immigrant integration policy. Rather, it proclaimed its task to be that of reviewing current policies in this domain, including immigrant integration as well immigration and welfare state policies in the context of ongoing internationalisation. Internationalisation was a focus of this new council, which also produced several other reports during this period on, for instance, the EU (WRR 2001a). This thematic programme was, however, different from WRR's more substantial policy agenda at the time of its 1989 report.

A thematic focus on internationalisation influenced the clear stance of WRR's report vis-à-vis the field of immigrant integration research. WRR adopted a transnationalist framing that linked immigrant integration to the 
ongoing process of internationalisation and set it apart from other frames with a more national perspective. Contrary to previous reports, WRR could not once again lean on issue-specific experts within its own organisation. As a result, the authors expended significant effort in examining scientific literature on the subject. ${ }^{31}$ In the initial stages of the report, the authors met with various researchers on immigrant integration. They were also supported throughout the project by researchers such as Kloosterman, who had held a part-time position in WRR for some time, and Entzinger. In this way, WRR became acquainted with the transnationalist frame that had developed in the context of a larger ongoing process of internationalisation. ${ }^{32}$ In fact, as prior observed, transnationalism emerged in this field as a consequence of a structural process of internationalisation and, in particular, growing involvement by European institutes, which induced researchers to look beyond their national models of immigrant integration and to engage in more international comparative research (Favell 1998, 2001; Lavenex 2005).

This demarcation of WRR's mandate involved a deliberate strategy of 'keeping politics out'. Its independent, authoritative position enabled WRR to fulfil a debunking role. In fact, WRR's advisory council, whose members included directors of several other institutes, including from SCP, encouraged WRR to use its independence as a means to discuss sensitive issues such as immigration and integration. The SCP director, who was later to become an important entrepreneur in this field, argued that WRR should take advantage of its independence to address this sensitive topic, something that SCP would be less capable of doing due to its relationship with the Department of Home Affairs. ${ }^{33}$

In 2000, in the midst of the development of the WRR report and following the second national minorities debate, immigrant integration emerged at the centre of public and political attention. For WRR, this was a moment to reinforce its strategy of 'keeping politics out'. It decided not to engage in the ongoing debates because the report would be published much later. Furthermore, it rejected the negative tone towards immigration and diversity that was evident in this debate. Ultimately, the WRR report would contain no reference to the article by Scheffer that had triggered the second national debate. In a memorandum to the council, the WRR project group argued, in reference to the assimilationist Scheffer debate, for a more international perspective.

The article [...] has led to a vivid debate about the success or failure of the integration of 'allochthones' in the Netherlands. It is striking that the positions were hardly nuanced, that all allochthones were treated as one and that policy responses have an ad hoc character; several elements were suddenly overemphasised in comparison to others. [...] With our report, we wanted to nuance this debate and 
present a more integral approach and, wherever possible and relevant, also involve the international debate about immigration and integration. $^{34}$

It is significant that WRR renounced ideas of an advisory request for a report on immigration policy in this period. Although the Minister of General Affairs formally issues advisory requests, there was debate at this time about a potential advisory request from the Department of Justice. Considering a new immigration law that was to be implemented in 2000, the State Secretary for Immigration at the Department of Justice showed particular interest in a WRR study on this issue and contemplated issuing an advisory request. ${ }^{35}$ The study was to address questions such as how to distinguish real (political) refugees from bogus (economic) refugees and look for explanations for why immigration to the Netherlands was so high. ${ }^{36}$ WRR responded negatively to discussions about this advisory request. It considered it too difficult to create an 'objective' explanation of reasons for migration and, more precisely, the choice to immigrate to the Netherlands. It also believed that such a report would deviate too greatly from the project, already underway, that focused mainly on cultural diversity. ${ }^{37}$ Moreover, it was feared that this topic would not be 'scientifically investigable', in part because of the political nature of distinguishing between economic migrants and political asylum applicants. ${ }^{38}$ Council members were fearful of an advisory request that would effectively put a political problem on WRR's plate. ${ }^{39}$ It was never issued.

This strategy of making of the report in a concertedly apolitical way, despite the rapid ongoing developments during its production, also reflected internal difficulties in achieving the goals set within WRR. Initially, WRR had planned to formulate a 'minimum of commonality" ${ }^{40}$ required for the functioning of an immigration society. At the beginning of the project, it was observed how 'the increase in ethnic diversity in Dutch society raises a lot of questions [...] [W]e have the impression that an important part of the answer can be found in cultivating specific general conditions' ${ }^{41}$ With this focus, the project group developed working titles such as 'cultural heterogeneity', 'multiculturalism' and 'coping with differences'. Later, it narrowed down this problem formulation to a more modest state. ${ }^{42}$ Instead of formulating its own normative framework for a minimum of commonality, the morality of the core institutions in society was taken as a normative starting point. In other words, a more sociological institutionalist mode of reasoning was adopted, somewhat similar to the 1989 WRR report, with a focus on the 'social state'. This was seen as a way of abandoning the idea of developing a substantive organising principle (i.e. a minimum of commonality) for a more procedural principle. ${ }^{43}$

One reason behind this more modest formulation was an internal disagreement about the broader normative framework. As a sensitive issue, 
immigrant integration led to considerable disputing within WRR, especially surrounding the Scheffer debate but also surrounding specific issues such as dual nationality and the economic evaluation of the costs and benefits of immigration. ${ }^{44}$ This made it difficult to agree on a common normative framework or to formulate clear policy recommendations on which all members could agree. These internal difficulties were an important factor as to why the report did not adopt policy recommendations as strong as those in previous ones.

Looking back, we could have perhaps gone a bit further in terms of recommendations. That our recommendations were eventually a bit 'softer' was also because this is a politically controversial issue, on which it is difficult to reach agreement within the project group and within the council. [...] You must have a very good argumentation and a good idea about the perverse effects of such an approach. ${ }^{45}$

By trying to keep politics out, WRR aimed to debunk or demythologise some prevailing national myths about immigrant integration in the context of internationalisation. ${ }^{46}$ Rather than being a 'report to government', this was more 'a report to society'. It contained a new perspective on immigration and integration rather than a new policy plan. ${ }^{47}$ It did not contain many concrete policy recommendations, 'but was rather aimed at introducing a change of perspective' ${ }^{48}$

Debunking involved, firstly, revealing the pervasive effect of migration and diversity as facets of the ongoing process of internationalisation on the structure of national institutions. It also involved a 'conceptual debate about concepts [such] as multiculturalism and integration' and a discussion about 'taboo topics such as differences between groups', including the issue of dual nationality and the relation between immigration and integration. ${ }^{49}$ WRR believed that the notion of multiculturalism had been troubled by normative debates on whether the Netherlands should be a multicultural society, rather than a factual debate about what cultural diversity meant for society. Furthermore, debunking meant that WRR diverged from the prevailing policy discourse. It avoided the concept of 'integration' because the notion reflected the dominant national discourse and stressed deficiencies on the part of immigrants, rather than the larger implications of diversity and migration. Instead, more analytical concepts such as 'incorporation' and 'participation' were used. ${ }^{50}$ Another myth that was addressed concerned the relation between immigration and integration, which had, thus far, been treated as largely separate policy areas, embedded in different institutional contexts. Whereas it had previously been accepted that integration policy was conditional upon immigration policy, WRR now argued that immigration policy should also be conditional upon integration policy. 
In an immigration society, immigration and integration policies were intimately entwined.

Thus, WRR dissociated itself from national policies, politics and the ongoing national debate on immigrant integration in an effort to debunk national myths about immigrant integration in the context of ongoing internationalisation. However, the timing of its report proved highly inopportune. Between the day the report was sent to the publisher (20 August 2001) and the day it was made public (24 September 2001), the terrorist attacks of $9 / 11$ occurred. Immediately following $9 / 11$, the council met to discuss whether changes should be made to the report; they decided no modifications were necessary. ${ }^{51}$ Although WRR had already chosen to dissociate itself from ongoing developments in public debate by not engaging in the 2000 minorities debate, this unfortunate timing meant that the report appeared in a very different problem setting than when the project began. In the words of one of the staff members, it had already been 'overtaken by problem developments such as the Scheffer debate and 9/11 by the time it was published, 52

\section{SCP: Boundary work within the nation-state}

SCP was another research actor to get more deeply involved in immigrant integration during the 1990s that now began advocating a more assimilationist policy approach. The institute had gained a structural position in the field of immigrant integration research as a data provider on the position of minorities. During the 1990s, it demarcated and coordinated its role in instrumental terms in the context of involvement in the new structure-induced equilibrium of the universalist approach of the integration policy. This, however, changed as SCP's position became steadily institutionalised in the 1990s.

Although SCP continued to be a provider of quantitative data, it came to define its task as being more than just instrumental. Its mounting authority in the domain enabled it to become less reserved, making more normative statements about immigrant integration. ${ }^{53}$ This was manifest in its more open advocacy of an assimilationist policy approach in 1998's 'Social and cultural report'. It was also illustrated by SCP director Schnabel's engagement in the public debate on immigrant integration. This more normative engagement attracted significant criticism, some of a moral nature. According to Schnabel (see Blok 2004b: 294):

My argument received a somewhat mixed response, because it was a very critical story: there is no multicultural society, nor would it be a good option. Somewhat on purpose, but perhaps also a bit naively, I used a rather sensitive word: I said that there is no choice but to choose for assimilation. [...] Because I had not been involved in this branch for so long, I did not realise that this was rather hard 
and unpleasant for some people. [...] I was rather shocked by the fierceness of the responses. [...] You find yourself quickly in situations about moral issues or moral decency.

This new role for SCP was, in sharp contrast to that of WRR, coordinated with ongoing developments in public and political debate. In fact, SCP believed it was meant to respond to shifts in public and political mood (SCP 2005: 7). Furthermore, its sharpening focus on social-cultural integration was linked with actors in the field of policymaking. This coordination occurred primarily via the involvement of SCP's director in the ministerial sub-council ('onderraad' in Dutch) that dealt with the topic of immigrant integration. ${ }^{54}$ In this way, SCP had direct access to the Prime Minister and other involved ministers, unlike WRR, which launches reports to the government but has no formal role in their policy follow-up. Furthermore, personal relations between $\mathrm{SCP}$ and the government appear to have grown in this period. SCP's director had a good working relationship with the minister responsible for integration policy until 2002. Moreover, one of the main authors of the minorities reports moved from a position at SCP to that of a research coordinator within the government directorate responsible for coordinating the minorities policy. ${ }^{55}$

Although SCP ventured beyond its traditional instrumental boundaries during this period, it served primarily in a functional relation to national policy institutes and national politics. ${ }^{56}$ Rather than debunking myths about immigrant integration on a national level, its role was to facilitate the development of integration policies on the national level.

\section{The Verwey-Jonker Institute: Boundary work of a private research institute}

Another research actor that was involved in this period was the VWJ. This institute was asked to carry out a policy evaluation study based on available scientific sources for the parliamentary investigative committee on integration policy. Later, it was also asked undertake additional research on more specific issues together with another private research institute $(\mathrm{QA}+)$. Given the VWJ's more substantial participation in the investigative committee and its focus on questions that concerned problem framing (to what extent policy was or was not successful), I will focus, in particular, on the institute's policy evaluation study.

The VWJ was selected through a public tender organised by Parliament's Research and Verification Office. The scientific sources study requested by the Blok Committee was to evaluate government policies based on an extensive evaluation of available literature on the integration policy and progress of the integration process. Moreover, the study had to be completed within two and a half months. The VWJ was selected for financial reasons, because of its expertise in this area ${ }^{57}$ and as it was also 
argued that the institute had had no past involvement with government policy (Blok 2004a: 15-16).

This selection criteria provides a clear indication of how the VWJ's tasks were defined. Within a short time and based on available scientific sources, it would have to evaluate the extent to which policy had been successful in specific areas. Other experts and institutes rejected the Blok Committee request because they felt they had been too deeply involved in policy themselves or the timeframe for carrying out research was too limited to allow for a meaningful scientific examination. One of those who rejected the request was Entzinger, having been deeply involved in policy developments in his work at ACOM, as well as serving as an author of the 1989 WRR report and an actor in 1994's policy succession minorities debate. ${ }^{58}$

An important factor behind the VWJ's decision to participate in the public tender was the fact that the research assignment was open - that there were no foregone conclusions about policy failure or success. ${ }^{59}$ The VWJ wanted to safeguard its integrity as a scientific research institute by allowing the research outcomes to vary and avoid providing scientific legitimacy to already formulated political conclusions. Although a parliamentary motion had originally asked for an evaluation of why policy had been so minimally successful - thereby defining policy as a failure - the ultimate question founding the VWJ study was more open-ended. A parliamentary working group established in response to this motion to make preparations for a research committee had reformulated the research question into a broader one: to what extent had policy failed or not (VWJ director in De Hart \& Prins 2005: 182).

In terms of coordination, the private status of the VWJ influenced its relationship with Parliament. As a relatively young institute, in need of assignments to establish its status and reputation, the VWJ had an important market incentive to accept the research questions formulated by the parliamentary working group. The opportunity to be involved at a time when the issue was so high on the agenda furnished an opportunity for the VWJ to strengthen its reputation, 'as researcher[s] must always have a particular relation to the dominant discourse. [...] Only if you discuss really cutting problems will people be willing to read the rest of the report' (ibid.: 185). This also meant that it had to accept the more substantive focus implicated in these research questions. The fact that all the research questions only concerned the social-economic domains of immigrant integration, omitting social-cultural issues, meant that there was already a specific 'framing' of the issue of immigrant integration going on. The principle-agent type of relationship between Parliament and the VWJ obstructed opportunities for this institute to discuss these questions in a normative way. The institute was also aware that its involvement in this controversial issue could prove problematic; however, it could not have anticipated the severity of the controversies to follow. ${ }^{60}$ 
Thus, the multiplicity of frames in the field of immigrant integration research was clearly reflected in the multiplicity of boundary work practices by research actors in the ongoing policy developments. WRR clearly demarcated its role from ongoing developments in public and political debate ('keeping politics out'), and coordinated its relation to policymaking in terms of debunking prevailing myths about national immigrant integration in the light of internationalisation. In contrast, SCP defined itself more and more through active engagement in ongoing debates about immigrant integration and coordinated its relations with national politics and policymaking in functional terms. Whereas the boundary work of WRR was aimed at reaching beyond national policymaking, SCP clearly remained within these parameters. Furthermore, the VWJ defined its dealings with Parliament in terms of a principle-agent relationship, in which it had only limited scope to negotiate the research assignment and whereby market incentives encouraged an acceptance of the commission.

\subsubsection{The field of policymaking}

The assimilationist policy pattern to emerge just after the turn of the millennium coincided with the sharp politicisation of immigrant integration. How did this politicisation affect the boundary practices of key policy actors in this period? In this context, I will examine the boundary work of the parliamentary investigative committee that was established following the 'long year of 2002', as well as that of the political actors and political entrepreneurs who were playing a key role in keeping immigrant integration on the political agenda.

\section{The Blok Committee: Boundary work of a committee under pressure}

The Blok Committee was established by Parliament after the controversial elections of 2002. A parliamentary motion was accepted that asked for a parliamentary investigation to examine why the integration policy had been so minimally successful. ${ }^{61}$ This motion revealed that Parliament regarded the integration policy thus far as a failure. As discussed above, the negative evaluation was based on an assimilationist perspective that stressed, in particular, the lack of policy results in the domain of social-cultural integration. Furthermore, it showed how Parliament sought a new impetus to the integration policy - it wanted to create a broad political basis for changes in the integration policy.

This committee's tasks gradually became narrower and more specific. An important element here was the broadening of the research questions it was to deal with. A parliamentary working group ${ }^{62}$ had reformulated the research problem in a freer way, allowing for broader conclusions; it made evaluating policy success or failure a part of the research problem. The purpose was to enable Parliament to assess the integration policy adapted 
over the past decades, including its intended policy goals and obtained policy results, as well as the consistency and coherence of policy in various areas including housing and recreation, income and labour and education. The committee would also have to provide 'building blocks' for a future integration policy. The assignment eventually formulated by Parliament was as follows: ${ }^{63}$

To enable the Second Chamber of Parliament to evaluate the integration policy of Dutch government over the past 30 years, to evaluate the aimed effects and factual results of this policy and to evaluate the coherence of policy on various policy terrains. Moreover, the research should provide building blocks for the integration policy to come. The questions that need to be answered by the temporary committee are:

- What integration policy has been conducted in the Netherlands over the past 30 years?

- What were goals and results of this policy in important areas such as housing and recreation, income and labour and education?

- Has there been a consistent and coherent integration policy in the various domains?

- To what extent can this policy, given its goals, be qualified as successful?

- Are there experiences in foreign cities with the integration policy from which our country can learn?

Once the investigative committee had been established, Parliament further specified the task. ${ }^{64}$ The committee's first task would be to commission a study of available references or 'sources' - only after completion of which it could then pursue its further activities. Initially, the literature study was to be completed in about seven weeks. ${ }^{65}$ However, the committee decided that this period was unrealistic, thus extending it by seven weeks. ${ }^{66}$ In the midst of this, the new centre-right government that was established in 2002 fell. However, following new parliamentary elections, the committee was re-established in February 2003, with more or less the same composition. ${ }^{67}$

Relations between the committee and the evaluation VWJ study were coordinated in such a way that the latter would be the starting point for the former. In fact, the VWJ Institute was asked to answer the same questions as those posed to the committee. This was crucial in that it not only provided the required information for the fulfilment of the committee's task, but also provided a means for coping with the tremendous political stress being put on this committee - because of the political sensitivity of the issue at hand, the committee was 'constantly under pressure'. ${ }^{68}$ The pressure manifested itself in political differences among the various parties represented in the committee as well as in political incentives to leak 
information to the media. ${ }^{69}$ This posed political threats to the research committee and political opportunities for parties wishing to distinguish themselves from the others. ${ }^{70}$ Surveying available sources provided a way of coping, by first examining 'the facts' that could tame the centripetal forces. $^{71}$

Based on the VWJ report, the committee would formulate its own conclusions during the second phase of the committee proceedings. ${ }^{72}$ First, a series of interviews was held with those people who had been involved in the national and local integration policies over the past decades. Two series were held - one closed and one open. The former aimed to 'test the findings from the sources study', to 'enhance knowledge and insight in the matter concerned' and to 'select the people that would be invited for the open meetings'. ${ }^{73}$ In total, 103 meetings were held with 145 people. The 'primary function' of the open meetings, again with a very large cast of actors - 86 meetings with 142 people - was to 'test the findings of the literature study in public' ${ }^{74}$ The interviewees included former ministers, civil servants from the local and national level, scientists, representatives from minorities organisations and also 'successful immigrant women'. Furthermore, these public hearings welcomed participation from all interested parties. Two large and two medium-sized cities were selected from across the country, with participation open to all. The goal of these public hearings was to heed the 'voice of the street' and, once again, to 'test the findings from the literature study'. ${ }^{75}$ The organisation of these public hearing was referred to as the "public conclusion" 76 and it furnished more fundamental input to the committee. Finally, based on the findings from the VWJ study, the committee decided to commission six more on several topics, including the labour market position of immigrants; the role of self-organisations, welfare organisations and interest groups; the emancipation of girls and women; the policy objectives in education; experiences in foreign cities with immigrant integration; and finally, the role of governments in immigrants' countries of origin. These studies were also commissioned from private research institutes, including QA+ and, once more, the VWJ.

Establishment of this parliamentary investigative committee was a political initiative to strengthen Parliament's control over the government's integration policy. An evaluation of the policy fiasco would provide a way for Parliament to free itself in this domain following the turbulence of 2002, and provide a new political impetus to the integration policy. However, political pressure, time constraints and committee's research design seem to have narrowed the committee's work. These factors contributed to the most central aspect of the study - an evaluation of scientific sources by the VWJ. The interviews, public hearings and additional studies that were conducted in the second stage mainly served the purpose of testing and refining the VWJ's conclusions. Parliamentary effort to set a new political 
course in this domain was therefore based on a study of scientific sources that had been conducted under serious constraints.

\section{Politics: Putting scientific credibility on the line}

The parliamentary initiative to provide new impetus to integration coincided with efforts by the new centre-right government coalitions of 2002 and 2003 to develop another approach to immigrant integration. Immigrant integration had shot to the top of the political agenda following the rise of Fortuyn, a series of focus events (such as 9/11 and morality-questioning incidents in the Netherlands spurred on by radical statements from a Dutch imam) and the continuous involvement of public intellectuals. Notable individuals were Scheffer and Ayaan Hirsi Ali, a female Dutch Parliamentarian of Somali birth whose critique of Islam attracted much attention in politics and the media. It was in this politicised context that the centre-right government took charge of the development of an integration policy 'new style'.

Political actors in this period defined their task as respondents to heightened feelings of anxiety about multiculturalism and, in general, the Dutch democratic establishment, which had manifested themselves in 2002 (Pellikaan \& Trappenburg 2003). For Fortuyn, public discontent about multiculturalism and politics were interconnected (Wansink 2004). An important aspect of Fortuyn's populist agenda, aimed against elitism in Dutch politics, was drawing attention to politics being deaf to the 'voice of the street'. According to Fortuyn, but also Hirsi Ali and Scheffer, politics ignored the alleged 'clash of civilisations' taking place within society because of its adherence to a supposedly benevolent multiculturalism. By avoiding political debates and maintaining taboos, politics was 'undemocratic' and elitist. Now, however, the centre-right government was clearly gaining a prominent position in public and political debates on immigrant integration.

In contrast to a politics of avoidance (De Beus 1998), an articulation function now emerged (VWJ 2004: 201), meaning that politics played a role in naming society's problems and feelings, articulating them in their actions to ensure the 'voice of the street' be taken seriously. Prins (2002b: 252) describes this in terms of 'hyperrealism', which, unlike new realism, involves not only politics eradicating taboos and speaking freely about the problems of integration, but an approach 'in which the courage of speaking freely about specific problems and solutions became simply the courage to speak freely in itself'.

Furthermore, these political actors and public intellectuals linked immigrant integration to broader concerns about national social cohesion and national identity. The debate about immigrant integration had become perceptively philosophical, in part due to the involvement of public intellectuals. It focused attention on matters of principle, such as freedom of 
expression and a clash of civilisations. Another emergent theme in this period concerned the freedom of religion in relation to principles such as freedom of expression, gender equality and separation of church and state. These matters were often showcased in the debate as symbolic incidents, such as wearing the Islamic headscarf while in public office, statements about Islam (by Fortuyn, among others) or some newsworthy event, such as in Tilburg in 2004 when an imam refused to shake hands with the Netherlands' female Minister of Integration and Immigration.

Defining politics' task of 'articulation' within policymaking evoked clear expressions of national orientation in political discussions on integration. In fact, immigrant integration became one of the central issues in a national debate about societal values and norms, which was formally called for the Prime Minister. Whereas the universalist approach of the 1990s had contributed to decentralisation, privatisation and the Europeanisation of elements of the integration policy, now a reverse trend was set in motion to put immigrant integration clearly at the locus of central government, especially in terms of policy formulation (Penninx et al. 2005). For instance, measures were taken to fortify the national government's role in civic integration courses. This included introducing standardised national exams and strengthening the symbolic meaning of Dutch nationality acquisition by organising ceremonial festivities and introducing stricter regulations against dual citizenship (De Hart 2004). In addition, a stronger link was established between integration and immigration policies and the stricter approach to immigrant integration was used as a means to restrict further immigration (Penninx et al. 2005).

These changes in how the task of politics was demarcated had consequences for the way relations with the field of research were coordinated. Politics became more selective in its use of research, adopting pick-andchoose strategies towards science (Penninx 2005). Whereas reports by WRR had marked fundamental policy turning points in the decades before, now the council remained largely ignored. The main conclusions of the Blok Committee, which had been established by Parliament itself, were especially ignored. Although Parliament accepted no fewer than 25 of the committee's 27 recommendations, it rejected the most fundamental conclusions regarding the success of the integration process. In contrast, government granted a more prominent voice to SCP. This was illustrated by the fact that, whereas prior policy documents were often directly related to earlier WRR reports, this time the first document in which the integration policy 'new style' was announced was related to the 2003 minorities report by SCP.

The credibility of researchers was discussed ever more openly. The Blok Committee and, in particular, the evaluation study it commissioned from the VWJ, became objects of fierce public and political controversy, even before the committee had published its findings. Internal wrangling in the 
committee drew public attention when member Ali Lazrak decided to leave the committee, under sway by his own Socialist Party (which, significantly, had taken the initiative for the parliamentary motion in 2002). In a flurry of media statements and revelations on the progress of the Blok Committee, the resignation of this member spurred controversy on the committee's research approach and, in particular, the VWJ report and its conclusion that policy had been relatively successful. Lazrak claimed that the committee should not have commissioned a study from this institute, as experts involved in this institute had been too closely involved with policy developments in the past. ${ }^{77}$ The authority of this institute was further called into question by the alleged political connections held by Jan Willem Duyvendak, one of VWJ's directors and a main author of the Blok Committee study. This expert had been involved with the leftist Green Party, which was now severely censured for its multiculturalist bias and accused of support for political correctness and taboos. In addition, the criticism concerned the committee's conclusion that the integration policy had been relatively successful. This conclusion immediately garnered responses from participants in the public debate, from involved public intellectuals as well as politicians who discarded such a conclusion as 'naive'. 78 Surprisingly little attention was paid to the more critical conclusions about the social-cultural integration of immigrants (VWJ director in De Hart \& Prins 2005: 185).

In the study for the Blok Committee we have observed spectacular progress of immigrants in the domains of housing, labour and education. [...] But we also concluded that the integration as a whole cannot be defined as successful. There are, after all, many other problems. Without the Blok Committee asking us for it, we have put these problems on the agenda, also to make that bridge to the broader debate. Nonetheless, we were attacked most on the positive part of our conclusion. Apparently, one is too quickly optimistic in these gloomy times.

Following criticism concerning the VWJ's study, in particular, controversy emerged about the entanglement of science and policy. Politicians would have overly 'delegated' the development of a political vision to scientists. A debate that had taken place earlier on a small scale, was now revived on a larger scale, criticising the immigrant integration research field for being too policy-oriented. ${ }^{79}$ Researchers were also criticised for having a multiculturalist bias and for operating primarily in the interest of minorities.

The committee report remained an object of public scrutiny after its publication. In parliamentary hearings, debate arose about whether the Blok Committee had 'measured' what it was supposed to measure. Questions emerged about the usefulness of an evaluation study when there was no 
consensus on the definition of integration. Hirsi Ali, who had since become a member of Parliament, claimed that 'there has never been a consensus on a definition of the word "integration" [and therefore] Parliament has given the research committee an assignment that was too vague'. ${ }^{80}$ Other parties argued, based on their frame of integration, that the committee report provided a thorough analysis of all the problems, 'but is too reserved when it comes to drawing the proper conclusions from all this'. ${ }^{81}$ Based on their own approaches to integration, various parties pointed to the report's 'blind spots', such as the place for religion - Islam, in particular - as well as criminality and a lack of attention to cultural issues. In addition, a representative from the Socialist Party that had initiated this committee asked how it could have come to its relatively positive conclusions about policy when it had been given an assignment to investigate why policy had failed. ${ }^{82}$ Finally, in the parliamentary hearings following the committee report, criticism continued over the choice for the VWJ to carry out the study. Soon criticism spilled over into the composition of the committee itself - for instance, one committee member, a former State Secretary for Education, had helped to investigate the extent to which her own policy had been successful.

In terms of boundary work during this era, the development of politics as an articulator of democratic beliefs on immigrant integration went hand in hand with mounting cynicism towards scientific research. Indeed, the use of scientific research became steadily selective and scientific credibility was more candidly discussed.

In sum, within the field of policymaking, there was a clear shift in how politics in policymaking was demarcated. Both Parliament, by establishing the Blok Committee, and the centre-right governments from 2002 and 2003 attempted to provide a new political élan to the integration policy. In terms of coordinating relations with research, there were some differences between the boundary work practices of the two actors. The Blok Committee privileged the literature study conducted by the VWJ. In contrast, the centre-right government adopted a more cynical attitude towards scientific research. Furthermore, in response to growing public controversy about the credibility of researchers, the government became very selective in its use of research.

\subsubsection{The construction of an engineering boundary configuration}

The field analyses of immigrant integration research and policymaking in this period reveal very different patterns of boundary work in both fields. How, then, did these patterns combine in terms of a specific research-policy dialogue? What type of research-policy nexus was constructed in this setting characterised by so much controversy? 


\section{Selective convergence between research and policy}

The interaction between research and policy seems to have been characterised by selective convergence as well as selective divergence in the relations between specific actors. On one hand, the boundary work of political actors and the SCP combined to produce a direct form of interaction. SCP defined its role as responding to shifts in public and political mood, and closely coordinated its activities with actors in the field of policymaking through its personal networks with policymakers and politicians, as well as its direct formal involvement in political decision-making. In this respect, it no longer demarcated itself in relation to policymaking in instrumental terms, but became more directly involved in policy framing. Government and political actors, such as the Minister of Home Affairs and later the Minister of Justice, also had a more direct relationship with SCP (than, for instance, with WRR). As an example, when social-cultural integration became an important issue on the public and political agenda, the government and SCP agreed to pay attention to the issue of social-cultural integration in the SCP minorities reports. As a result, SCP was more directly involved in policymaking. This close relationship between SCP and policy actors was indicated when the Minister of Justice, for the first time, elaborated the contours of an integration policy 'new style' in a letter to Parliament. It was issued together with the 2003 SCP minorities report.

The interaction between research and policy was also rather direct in the case of the Blok Committee and the VWJ. The former attributed a prominent position to the latter in drawing up its own report. By delegating the same questions to this institute as those posed to the committee - including the inherently normative question of whether the policy had been successful or not - it made no attempt to establish a strict distinction between the role of the VWJ and its own role as a parliamentary investigative committee. On its part, the VWJ also had no difficulties engaging itself so directly in the evaluation of the committee, in part because of its private status.

On the other hand, this convergence seems to have been selective, as signalled by amassing political cynicism towards scientific expertise and reluctance to use scientific research. For instance, the credibility of the VWJ report and, more generally, the Blok Committee became the subject of heated public debates. Furthermore, the 2001 WRR report went largely ignored. In fact, WRR also tried to dissociate itself from ongoing public and political developments. From an enlightenment model of researchpolicy relations, it attempted to debunk the public and political debates that had emerged since 2000, brining in a normative perspective of internationalisation that conflicted with the more national mode of policymaking prevalent in this period. 


\section{Political primacy}

This period provides indications of political primacy in the mutual relations between research and policy. For instance, developments in the field of policymaking after 2000 had an important effect on the research carried out by SCP. More than ever, policy actors were able to ignore research if it developed a frame other than the assimilationist one that became dominant in policymaking in this period. This is certainly what happened with the 2001 WRR report, but also in the case of the VWJ report where only the instrumental conclusions were adopted.

This political primacy was related to the politics of articulation that had emerged specifically after 'the long year of 2002'. Whereas the culture of depoliticisation in previous episodes had helped legitimise policies with the support of scientific expertise, an emphasis on politics' democratic responsiveness and a growing cynicism towards research signalled that political risks could be at stake. This became particularly clear when the Blok Committee, presumably endeavouring to use research to coordinate political consensus among its members. However, rather than offering a way to resolve political controversy over immigrant integration, the involvement of these scientific researchers instead became contentious.

The selective convergence between specific research and policy actors, along with the aforementioned signs of political primacy, points to an engineering model of boundary configuration. This involved the engineering of a new assimilationist approach to immigrant integration driven primarily by political developments in this period, making selective use of scientific research that could contribute directly to the framing of such an approach. This engineering model involved political actors with a primary role in developing the assimilationist approach following 'the long year of 2002', including the political leadership of the Department of Justice that had now become the coordinating department and also including SCP, which was clearly stretched beyond its instrumental task to one more clearly advocating assimilationism. It left out other actors that advocated alternative frames, such as WRR, which favoured a more transnationalist approach.

\subsection{Engineering and the selective co-production of research and policy}

Within a setting characterised by open controversy regarding both the framing of immigrant integration and a 'proper' division of labour between research and policy, a very selective engineering type of research-policy nexus emerged. Yet, how did this nexus contribute to the assimilationist turn in Dutch policies to occur in the new millennium? 


\subsubsection{The structural effects of engineering}

The first step in analysing the impact of this nexus on research and policy developments is to examine its effect on the structural equilibrium of the 1990s. Although there had been no heavily institutionalised research-policy nexus, as was the case in the 1980s, the next decade's universalist approach was supported by specific actors. So did the engineering nexus then constructed mobilise negative or positive feedback towards this equilibrium?

\section{Engineering and negative policy feedback}

The research-policy nexus seems to have played only a marginal role in the positive feedback process that led to the integration policy 'new style'. The process was driven primarily by macro-political developments - specifically in 2002 - and, in this issue domain, by developments on the policymaking side of the nexus, i.e. the context of the national minorities debate of 2000. The structure-induced equilibrium of the universalist approach in integration policy persisted at least until 2000, when the second national minorities debate emerged. Until then, immigrant integration did not rank highly on the public and political agenda. This was illustrated when WRR announced in 1997 that it would develop a new report on immigrant integration. The initiative was received with sharp cynicism on the part of DCIM, which saw no need to adapt the current universalist approach. ${ }^{83}$ The government maintained its approach, especially in view of evidence (provided by, among others, $\mathrm{SCP}$ ) that the social-economic position of migrants was improving considerably.

SCP, which was involved in the structure-induced equilibrium of the integration policy as a provider of instrumental data, contributed to the softening-up process that eventually led to the developments after 2000. Before and during the national minorities debate, SCP was one of the advocates of a more assimilationist approach. This was a consequence of the institutionalisation of SCP's position, which allowed it to go beyond being purely instrumental. In particular, SCP seems to have reinforced the trend towards assimilationism. This can be explained by SCP's functional position within the national government apparatus, which included responding to shifts in public and political mood.

The 2001 WRR report failed to generate positive feedback, in contrast to its previous reports, which had marked turning points in the development of immigrant integration policy. In the run-up to publication, WRR was met with cynicism on the part of a government seeing no need for policy change. This was superseded by a heightened sense of urgency by the time the WRR report was made public. However, the strongly altered problem context had made the report irrelevant. According to the chairman of the WRR project group: 
this report could have been considered quite critical of policy issues, until 9/11. Afterwards, it has mostly been regarded as 'too soft' a report, because then the discourse had become much harder in a relatively short period. Therefore, the nuance that we had developed in our report was now seen as too nuanced. ${ }^{84}$

The national minorities debate in 2000 and 'the long year of 2002' in Dutch politics were the most direct causes of the shift in attention from social-economic to social-cultural facets of immigrant integration. It was in this period that the bureaucratic research-policy nexus, which had sustained the structure-induced equilibrium of the integration policy in the 1990s, was replaced by the engineering research-policy nexus, with political primacy and more convergent roles of research and policy. The Minister of Home Affairs, together with SCP, organised more systematic attention to be given to social-cultural integration in response to the national minorities debate. Subsequently, the centre-right coalition continued to coordinate research on social-cultural integration, doing so with SCP and several other research institutes.

Engineering was thus functional to scientific research in the development of a more assimilationist policy approach in response to political developments after 2000. As such, it was a source of negative, rather than positive, feedback. Although engineering itself did not cause the changes in the structure-induced equilibrium of the $1990 \mathrm{~s}$, it did contribute to the development of a new structure-induced equilibrium.

\section{The engineering of research on social-cultural integration}

Engineering involved selective use of expertise functional to the development of a new structure-induced equilibrium in the policy field. In the field of research, it called attention to social-cultural topics, such as socialcultural integration, criminality and social cohesion. The Department of Justice played a central role in engineering research in this direction. While this strengthened the position of SCP, it also involved more research institutes with a functional relationship to the state apparatus. Various institutes now published reports on immigrant integration, including SCP with its (then biannual) minorities reports, the annual report by the CBS, 'Allochthonous in the Netherlands' and the ISEO with its regular integration monitors. The Department of Justice wanted to achieve better integration of these various studies. This led to the 'Annual report of integration' in 2005, a combined effort by SCP, CBS and the Scientific Research and Documentation Centre (WODC) of the Department of Justice. ${ }^{85}$ The project, however, turned out to be a one-off, as it proved too difficult to combine the various institutes' perspectives into a single report. In 2006, WODC and CBS presented an integration map showing key indicators of the position of 
immigrants in various areas. In the following year, SCP and CBS issued a more 'policy-oriented scientific [annual] report' (SCP 2006b: 13).

These studies covered social-cultural facets of immigrant integration as well as more traditional social-economic ones. For its 2003 minorities report, SCP had already decided, together with the Department of Justice, to include the topic of social-cultural integration. The integration map developed by WODC and CBS also included themes such as criminality and points of contact between immigrants and natives. The combined 2005 annual integration report included the themes social-cultural integration, liveability in concentration areas, youth and criminality, the position of women and public perception of the multi-ethnic society. In addition, 'traditional' themes such as labour and income, education, language apprehension, civic integration and demography were also covered in this report.

Another aspect of the engineering boundary configuration was that it allowed government to selectively interpret or ignore scientific research. This was the case with the 2001 WRR report, because of its transnationalist framing, but also with the VWJ's study, because it only focused on the 'traditional' social-economic facets of immigrant integration. Aided by the mounting cynicism towards scientific research overall and notably by public controversies on immigrant integration research, this engineering configuration facilitated government efforts to develop a new policy approach based on political factors. It also furnished the government with an opportunity to ignore scientific claims contending for the new 'assimilationist' approach.

As illustrated, the engineering boundary configuration was pivotal in policy and research frameshifts during this period, though as a source of negative rather than positive feedback. The policy frameshift that occurred after the turn of the millennium was a direct consequence of political developments such as 'the long year of 2002' and the national minorities debate of 2000. The engineering configuration had a functional role in establishing a structure-induced equilibrium around the new assimilationist approach. Engineering stimulated research on social-cultural facets of immigrant integration. In particular, it reinforced the position of research actors with a direct and functional relationship to government, such as SCP, but also gave weight to new actors such as WODC and CBS.

\subsubsection{Engineering, assimilationism and transnationalism}

In the policy field, the engineering configuration was mainly a source of negative feedback in establishing the new policy approach that was set by political actors. But did engineering also contribute to the rise of assimilationism? We have seen how it encouraged the rise of research into socialcultural facets of immigrant integration. However, as observed in chapter 4 , this period also marked the rise of transnationalist research. What was 
the relation between this engineering configuration and the rise of transnationalist thinking in this period?

\section{The engineering of a national perspective on immigrant integration}

The political events following the turn of the millennium connected the issue of immigrant integration to broader concerns about national identity, norms and values. For instance, Fortuyn turned immigrant integration into a symbolic issue for a broader populist agenda that was anti-elitist, antimulticulturalist and anti-Europeanist. This issue linkage on the national level meant that the call for an assimilationist policy approach stood, at least to some extent, apart from developments in the issue domain itself. Rather, it became a symbol of the populist struggle against the alleged political correctness of the Dutch political elites and against the political denial of popular dissent about the effects of immigration and Europeanisation on social cohesion.

In the political arena and public debate, public intellectuals who followed Fortuyn often made the claim that one has to be able 'to say the things we have all been thinking' (Holsteyn \& Irwin 2003: 62). From this populist perspective, a properly functioning democratic state apparatus should give credence to popular dissent and not ignore the 'voice of the street'. This has been referred to as the 'articulation function' of politics (VWJ 2004: 201). Indeed, in political debates following the contention surrounding the Blok Committee, politicians pressed the point that these controversies had, in fact, helped politics 'beyond the phase of denial' and had established a general feeling that 'disinterest and lack of commitment should now belong to the past' ${ }^{86}$ Following the committee report, most political parties presented papers in which they developed their position on a reformulation of the integration policy. According to critics, this articulation function involved a sort of hyperrealism, 'in which the courage of speaking freely about specific problems and solutions became simply the courage to speak freely itself' (Prins 2002b: 252). Hyperrealism replaced the old political correctness with a new political correctness concerning 'saying something positive about the integration of immigrants, which would be naive and would mean ignoring the problems'. ${ }^{87}$

This linkage to national political issues invited an assimilationist framing of immigrant integration by drawing attention to social-cultural integration rather than social-economic participation. The engineering boundary configuration allowed government to develop this assimilationist approach despite resistance from other involved actors. Generally, this resistance involved researchers who believed in differing frames but, for instance, the Association of Dutch Municipalities also offered stark opposition to the assimilationist approach (Municipalities 2003: 7-8). Adopting a clear political primacy and 'picking-and-choosing' those strands of expertise to support 
the new approach allowed government to engineer the assimilationist integration policy 'new style'.

\section{Engineering and frame conflicts in the scientific field}

Engineering of an assimilationist approach to immigrant integration in connection with national political developments further reinforced the fragmentation of the research field in terms of problem framing. It contributed to the rise of immigrant integration research with assimilationist frames, such as that forwarded by SCP and other institutes with a functional relationship to government, including WODC and CBS.

Whereas the engineering configuration on the national research-policy nexus was particularly susceptible to research of the assimilationist type, the research-policy nexus on local and European levels seems to have provided opportunities to develop other research frames. For instance, Penninx, an established researcher in this domain since the 1970s, observed that he (and his Institute for Migration and Ethnic Studies, or IMES for short) increasingly relied on local and European research funding (Penninx in De Hart \& Prins 2005: 183). Local governments gained more prominence in integration policy design during the 1990s due to territorial decentralisation. This led to local governments' rising demands for research, often aimed at coping with concrete problems of immigrant integration, rather than larger national symbolic issues. An illustration of research responding to local demands was a study of integration at the neighbourhood level funded by the City of Rotterdam (Duyvendak \& Veldboer 2004).

European institutes' involvement in research funding and the ongoing internationalisation of academic research created a different demand for expertise. Europe's growing participation created new opportunities and new 'structures and incentives for researchers seeking to probe these "problems of Europe" (Geddes 2005: 266). As yet, the EU had little means for international policy coordination, especially in the domain of integration, as the subject remained a resilient national issue. As such, the assignment of research was a so-called 'soft means for coordination'. The cooperation of researchers, then, could help construct migration and integration as 'problems of Europe'. '[T]he identification of problems of European integration can enhance the perceived relevance of the European dimension and contribute to arguments for closer integration and more power for supranational institutions' (ibid.: 267). This often involved international comparative research, which helped extend the debate on immigrant integration beyond the scope of the nation-state (Favell 2005). The recent expansion of European research funding for issues of migration and cultural diversity in the Seventh Framework Programme suggested that Europe was co-opting research as a soft measure of policy coordination. 
Frame conflicts resulted from this fragmentation of the immigrant integration research field. A heated controversy unfolded when one of the leading Dutch journals in this field, Migrantenstudies, invited a number of researchers to respond to the 2001 WRR report. ${ }^{88}$ The document was positively received by various researchers, including in a publication on the structural relation between immigration and the social welfare state (Entzinger \& Van der Meer 2004) and in a special booklet by the Scientific Bureau of the Social Democratic Party entitled 'The transnational Netherlands' (Becker, Van Hennekeler, Sie Dhian Ho \& Tromp 2002). However, Koopmans, then a senior researcher at the Social Science Research Centre in Germany, also wrote an article in the special issue of Migrantenstudies. In it, he compared the position of migrants in Germany with those in the Netherlands, thereby illustrating how WRR provided too positive an image of Dutch integration policies. According to Koopmans, Dutch integration policy had clearly failed, primarily because of its persistent tendency to reify cultural differences (as a legacy of pillarism). In a subsequent issue of Migrantenstudies (Böcker \& Thränhardt 2003) and in other journals (Snel 2003b), this claim was rejected and denounced as unscientific due to a number of methodological problems concerning comparative design. In a response, Koopmans (2003), clearly lifted this criticism to the level of problem framing, attributing the intense reactions his article promoted to a self-sufficiency among researchers concerning the Dutch multiculturalist and universalist approach.

Thus, the controversy over policy failure or success also entered the field of immigrant integration research. It piqued the interest of the Minister of Integration and Immigration of the first centre-right government in 2002 (Böcker \& Thränhardt 2003: 33). Influenced by the controversy surrounding the Koopmans article, SCP carried out a comparative study on the position of Turks on the labour market in the Netherlands and Germany, published in 2006 (SCP 2006a). In this study, SCP concluded that the labour situation of Turks was indeed weaker in the Netherlands than Germany. However, it claimed that this was due to differences between the two countries' Turkish migrant populations rather than to different policy approaches.

We have seen, then, how the engineering research-policy nexus at the national level contributed specifically to the development of an assimilationist framing in both policy and research. In this way, the engineering configuration also contributed to a national framing of immigrant integration. At the same time, the research-policy nexus at the local and European levels created opportunities for other frames of immigrant integration, focusing either on more local concerns or encouraging a greater European perspective beyond the nation-state. Therefore, the engineering configuration at the national level and the emerging research infrastructures at the 
local and European levels contributed to further fragmentation of the research field in terms of problem framing.

\subsubsection{Engineering and frame reflection?}

Beyond the engineering nexus' impact on both the fields of research and policy, it is important to look as well at whether it did in fact promote critical dialogues. And if so, did it make for exchanges that were open, empathic, reflective, pragmatic and trustful?

\section{Opportunities and constraints for frame reflection}

One factor that could have contributed to frame reflection in this period was the expansive scale of debate. More than ever, an open debate on immigrant integration emerged in the media and in politics. This drew in many new actors, into the political arena as well as the public debate. On one hand, the engineering boundary configuration seems to have contributed to this openness by enabling a political primacy that carried the discussion beyond researchers and policymakers who were involved in the prevailing policy monopoly. On the other hand, it also constrained the scale of debate by ruling out specific actors from the scientific field. The factvalue dichotomy delegitimised the involvement of researchers in the debate on problem framing because researchers would have to stick to the provision of data.

Another condition that could have contributed to frame reflection concerned the multitude of frames present during this period. Whereas in the 1980s there had been one dominant paradigm, which was rivalled by at least one alternative paradigm in the 1990s, now there were actors propagating universalist, multiculturalist, transnationalist and assimilationist frames. The engineering configuration contributed to the rise of the assimilationist frame in both policy and research. The other frames were sustained by research-policy relations, notably at the local and European levels.

Despite the presence of various frames, there were few instances of 'empathy', where actors tried to put themselves in the shoes of others who believed in different frames. This is illustrated by the controversy over policy failure or success, which had raged in the policy field as well as in the research field. This controversy digressed into a 'dialogue of the deaf' rather than promoting empathy in terms of understanding how and why other actors evaluated policy differently and adopted different frames. A dialogue of the deaf was clearly present in the public and political debate surrounding the Blok Committee and its conclusions regarding the success of the integration process. Actors involved in these discussions selected different data and it interpreted it uniquely according to their perspectives; actors with a universalist frame concluded that policy had been successful, 
whereas those with an assimilationist frame found that it had failed. This debate never achieved the level of problem framing that could have offered a way out of the dialogue of the deaf. Similar dialogues of the deaf emerged surrounding the 2001 WRR report and the Dutch-German comparison drawn by Koopmans.

Besides the limited accessibility and lack of empathy, there seems to have been only minimal critical reflection. Despite many instances during this era when frames collided, rarely did this lead to a critical examination of the consistency or coherence of the frames themselves. Often, frame conflicts produced controversy about the involved actors, questioning their credibility or even their morality. For instance, the frame conflicts surrounding the report by the VWJ and WRR led to a public enquiry into the scientific credibility and authority of these institutes and the people involved therein. Intense boundary struggles that increasingly took place in the open during this period contributed to this lack of critical reflection. The controversies over science-policy boundaries concealed the underlying issues about problem framing. The engineering boundary configuration also formed an impediment to critical reflection, selecting evidence that could support the assimilationist frame and ignoring possible counterevidence. The emphasis on a fact-value dichotomy in boundary discourse further inhibited critical frame reflection by constraining the task of scientists to the provision of data and inhibiting their influence as far as problem framing went.

Furthermore, reflection was inhibited by a lack of pragmatism or, put in another way, actors' lack of will to adapt their frames in response to confrontation with other frames. The politics of articulation - or, according to others, hyperrealism - legitimised the dominance of the assimilationist frame. This lack of pragmatism was also caused by the highly symbolic issue linkage between immigrant integration and populist concerns about elitism and Europeanisation. As such, the debate about immigrant integration was, at least to some extent, separated from developments in the problem situation itself and connected instead to larger symbolic issues. This was also illustrated by an absence of concern for policy practice and policy implementation, thus creating a widening gap, especially between national and local policies.

Finally, trust between actors in the scientific research and policy fields in this period was limited to a small number of actors sharing the same or similar problem frame. Moreover, it was confined to actors that had a functional relation to the state apparatus, such as SCP, CBS and WODC. Convergence in science-policy relations only occurred in the context of relations between state-associated actors who shared similar approaches. Other actors, sometimes associated with other levels of government, were left out of the small network of trust that sustained the engineering approach. In fact, the discursive fact-value dichotomy seems to have served 
mainly to rule out actors in the scientific field with values other than the dominant policy's, regardless of the fact that the 'data providers' involved in the engineering of assimilationism clearly also adhered to specific values. As such, the fact-value dichotomy marked a division between networks of trust and distrust.

\section{The Blok Committee and the Verwey-Jonker Institute: Missed opportunities for frame reflection?}

The proceedings surrounding the Blok Committee and the study by VWJ offer a clear illustration of how and why frame reflection failed in this period. In both the research design and the way the proceedings of the parliamentary investigative committee actually unfolded, there were several missed opportunities for frame reflection.

Parliament's motivation for establishing this investigative committee providing a new political impetus to the integration policy - may in itself have indicated willingness to reflect upon policy framing. However, an important opportunity for reflection seems to have been missed at the early stage of formulating the research problem. The parliamentary motion subdued notions of empathy and critical reflection towards alternative frames by concluding that policy had been 'insufficiently successful'. This substantive conclusion suggests that the parliamentary initiative to establish the committee had already adopted a particular problem framing, one based on the premise that the committee would have to examine why policy had failed to become such a fiasco and how 'building blocks' for a new policy could be developed.

In this respect, there was an inherent tension between this parliamentary initiative and the new centre-right government coming to power some time after the motion was accepted. In an attempt to improve its relative information position in relation to government, Parliament took a more substantial initiative in terms of policy development. On one hand, this showed the broad parliamentary commitment to policy change in this domain. On the other hand, it created a tension with the new centre-right government, which included parties that had spoken out on immigrant integration during the elections, and also presented new substantial plans for policy development.

Furthermore, the parliamentary working group that reformulated this motion into formal research questions for the Blok Committee further constrained opportunities for critical frame reflection. Although it broadened the research questions by allowing for an evaluation of policy success or failure, it also adopted an implicit problem frame by selecting mainly social-economic domains as targets for the parliamentary investigation. As previously observed, this selection revealed a universalist frame by omitting the social-cultural issues that had then become prominent in public and political debate. This selective formulation of the research questions 
showed that the committee was not established to reflect on alternative frames but instead, to evaluate policy and contribute to policy development based on a specific (universalist) approach.

Another element of the research design that constrained frame reflection concerned the study of scientific sources commissioned from the VWJ. As observed above, the Blok Committee delegated all the research questions to the VWJ, including the normative question concerning policy success or failure. Based on its frame (also universalist), the VWJ examined scientific sources and concluded that policy had been relatively successful. The VWJ's exclusive role meant, however, that only one frame was included in the analysis of sources and the evaluation of policy success or failure. Had the Blok Committee asked advice from several institutes, or from one institute with the explicit instruction to use various alternative problem frames, it would have been able to reflect on these alternative frames within the committee. Furthermore, the task of scientific research would have been to facilitate frame reflection within the politically constituted Blok Committee; the broad political position of the committee would then have been to undertake frame reflection by employing a type of scientific involvement that articulated diverse, alternative models.

The political pressure on the Blok Committee formed a further impediment for frame reflection. This tension manifested in the form of sharp political differences on immigrant integration, as well as political incentives for the party representatives to seek publicity for themselves. At first, asking for expert advice seemed to offer a way to cope. Eventually, however, it appeared to be at odds with the frequent questioning of scientific authority and expert involvement in problem framing. Furthermore, the stress exacerbated already existing time constraints on the committee as a whole and frustrated, specifically, the study of scientific sources. A lack of time clearly limited opportunities for including more frames in this study.

Thus, as the case of the Blok Committee illustrates, the frameshifts in this period were not purely products of frame reflection. Despite the multiplicity of frames (especially in research) and the expanding scale of the debate, most other conditions for frame reflection were not met. The engineering boundary configuration contributed to keeping the scale of debate, and also trust, confined to a limited network of actors, who generally shared a comparable frame and were closely associated to the state apparatus. It also failed in terms of empathy. Frame conflicts (such as the controversy over policy success or failure surrounding the Blok Committee) often digressed into a dialogue of the deaf, whereby actors with different frames selected different data or interpreted data differently, rather than leading to a critical debate on the level of problem framing. Furthermore, critical reflection was inhibited by the tendency to a pick and choose evidence (from $\mathrm{SCP}$ ) in an effort to reinforce the assimilationist policy framing and ignore counterevidence (from WRR). The boundary struggles associated with the 
engineering configuration also shifted attention from the involved frames to the credibility of involved actors. Finally, the politics of articulation and the increasingly symbolic nature of the debate on immigrant integration diminished the willingness of actors to adapt their frames. In fact, as the case of the Blok Committee showed, actors often relentlessly held on to their frames. This is demonstrated not only by reluctant responses to the Blok Committee, but also the committee itself and the VWJ's persistence in holding on to a specific problem frame.

\subsection{Conclusion}

This third episode in the development of immigrant integration research and policy in the Netherlands clearly reveals the difficulties in organising research-policy dialogues in social settings characterised by ardent controversy. Just after the turn of the millennium, immigrant integration became highly politicised in one of the most dramatic periods in Dutch post-war political history. Murdered politician Fortuyn transformed immigrant integration into a symbol for the democratically unresponsive and politically correct political elite in the Netherlands. A series of national and international incidents contributed further to popular and political concern about the state of immigrant integration. It was this politicisation that drove the assimilationist turn in Dutch immigrant integration policy, not unlike many other Western European countries during this period.

In this conflicted setting, the role of research in policymaking also became severely contested. This underscores Gusfield's (1976) claim that the 'culture' or the framing of problems are inherently connected to the 'structure' of these problems; thus, when framing is contested, the structure can be expected to be contested as well. In this period, the credibility of immigrant integration researchers, who had been so heavily involved in the construction of policies in the past, was openly put on the line. Researchers were held responsible for the elitist style of policymaking in this field and for their contribution to the multicultural model that was now so widely discarded as a failure (regardless of the fact that the model had already been abandoned in formal policy discourse a decade earlier). This became particularly evident in the parliamentary investigative group known as the Blok Committee that fruitlessly attempted to forge a consensus by commissioning a state of the art of available research findings - a decision which became the subject of intense public and political scrutiny.

The technocratic and enlightenment models of research-policy relations were now strongly rejected. Politicians were called upon to develop a sharper political vision and maintain political primacy. In this respect, a socalled 'articulation function' emerged in politics, meaning that politicians considered it their task to respond to popular concerns about immigrant 
integration when developing policies that are democratically accountable, rather than having their policies grounded primarily on the advice of academic or ethnic experts. In this setting, the use of scientific knowledge and expertise was very selective and primarily instrumental: a pick-and-choose strategy emerged in which government selected those strands of expertise that were functional to the legitimation of the new assimilationist policy. This type of nexus can best be described in terms of the engineering model, with political primacy and a selective and functional utilisation of research in the engineering of specific policy programmes. This explains why the third WRR report on immigrant integration remained largely ignored: it adopted a very different problem frame and also contained much more conceptual, rather than instrumental, policy advice. At the same time, SCP's position became more prominent thanks to its open advocacy of a more assimilationist approach and its focus on issues of social-cultural integration.

This episode illustrates perhaps most clearly how research-policy dialogues on intractable controversies like immigrant integration can digress into dialogues of the deaf. Both the fields of research and policy had a pronounced multiplicity of problem frames. This makes it difficult to organise dialogues across frames. For instance, evaluation of the integration policy as a failure, which provided the basis for the establishment of the parliamentary investigative committee, was driven primarily by a social-cultural (assimilationist) frame of immigrant integration. However, the VWJ study that provided the basis for the committee's conclusions evaluated the integration process that was driven by a more social-economic (universalist) frame. At the same time, WRR framed immigrant integration in a more transnationalist way. This means that these actors had very different evaluations of policy as either a success or a failure, involving very different ways of selecting and interpreting available evidence.

Critical frame reflection would, according to Rein and Schön (1994), be an important way to transcend dialogues of the deaf. However, the engineering boundary configuration seems to have inhibited frame reflection, as it was strategically designed for establishing an assimilationist policy frame by coordinating research functional to this aim and ignoring research with alternative frames. It enabled government to promote and exploit selective research on social-cultural integration within a national context and ignore research that challenged such a national perspective on immigrant integration. In the field of research, the national research-policy nexus contributed to further fragmentation in terms of problem framing. This was also encouraged by the emergence of an alternative research-policy nexus on local and European levels. Ultimately, the engineering approach obstructed a critical frame reflection by constraining the debate across the research-policy nexus to a small group of actors who shared a similar problem frame (assimilationism) and ignored alternative frames (selective use of research, cynicism towards science). Moreover, these actors were not 
pragmatic; instead, they followed a logic of articulation, responding to political developments during this period, and their trust was confined to a limited number. 



\section{Dutch exceptionalism? Immigrant integration research and policies in France, Germany and the United Kingdom}

The Dutch case has revealed very specific patterns of research-policy dialogues over the past decades. First, it has shown unique relations between the structure of the research-policy nexus and the framing of immigrant integration. In this sense, it reflects Gusfield's (1976) premise that the structure and the culture of public problems must be considered inherently connected. The technocratic symbiosis of the 1980s helped keep policymaking behind closed doors, enabling a 'logic of minorities' in problem framing that was relatively insulated from broader societal developments. The enlightenment configuration of the early 1990s helped punctuate this symbiosis and link immigrant integration to these broader societal developments. This enabled a 'logic of equity' in problem framing that stressed the commonalities of migrants as citizens rather than group-specific cultural particularities. Finally, the engineering configuration from 2000 onwards permitted a cherry-picking of knowledge and expertise in the highly politicised setting that drove the assimilationist turn in Dutch policy during this period.

Secondly, the Dutch case has displayed a trend of growing political primacy and declining belief in rational societal steering based on scientific policy advice. Technocratic and enlightenment models of research-policy relations were rejected after the turn of the millennium, making way for models in which research was more serviceable to the prevailing political agenda. In some respects, a widening gap between immigrant integration research and policy has also been observed, especially in terms of differing views at the level of problem framing. The highly institutionalised research-policy nexus playing a central role in constructing the famous Dutch multicultural model in the early 1980s had already been deinstitutionalised by the early 1990s. At the same time, however, a new sort of nexus - of an engineering, if not bureaucratic, nature - was erected at a more instrumental level. It involved, in particular, data provision on key policy facets and the provision of knowledge claims helping to legitimise the new policy discourse that emerged after the turn of the millennium. Thus, there was a distinct trend away from science-led models such as technocracy and engineering, to more politics-led ones such as engineering and bureaucratic models. 
The objective of this chapter is to examine whether these patterns can also be identified beyond the Dutch case. Is there a 'Dutch exceptionalism'? Or can the relations found between the structures of research-policy dialogues and the frames of immigrant integration, as well as the trend away from models of societal engineering to models with a stronger political primacy, be generalised beyond the Netherlands? To endeavour to answer these questions, analysis will be drawn of research-policy relations and their role in the framing of immigrant integration in France, Germany and the UK.

The aim here is to reach beyond 'models thinking', which would only emphasise national differences rather than similarities and would inevitably contribute to the further reification of national models of integration. Instead, the focus will be on the construction of these models and, in particular, the role of research-policy dialogues in this process. Comparing countries' different models of integration involves selection on the dependent variable. Instead, the selection of these countries was based on known differences in their national styles of research-policy relations (see Boswell 2009; Halffman 2005). In Germany there are traditionally sharp boundaries between research and policy, with the division of labour between both fields often highly formalised. This applies less to France and the United Kingdom. In France, there are often close personal connections between researchers and politicians/policymakers, and there is a set practice of political involvement by public intellectuals. In the UK, too, the division of labour between both fields is much less codified, often involving personal cross-boundary networks and a form of authority and credibility that is highly vested in individual people.

For each country, we therefore first examine how immigrant integration has been framed in the research and policy fields in these countries. Secondly, we analyse the frames' construction - notably, the role of research in the construction. Finally, as in the Dutch case, we assess the extent to which research-policy dialogues in these countries have contributed to critical frame reflection. What can be learnt from these country studies in terms of how to organise frame-critical research-policy dialogues?

\subsection{France}

\subsubsection{The French model(s) of integration}

France - more so than the Netherlands - is an experienced country of immigration. Throughout the twentieth century, France witnessed significant immigration and was much less a country of emigration than, for instance, the Netherlands (Weil 1991; Schain 1999). Even though France developed into an immigration country earlier than most other European nations, immigration has not become part of its founding myth (Tilly, Noiriel \& De 
Laforcade 1996). In other words, France is an immigration country, but not an immigrant society or a so-called nation of immigrants as, for example, the United States.

France has become internationally renowned for its republican model of immigrant integration, characterised by assimilationism and individualism and its reluctance to define immigrants in racial, ethnic, religious or cultural terms (Weil 1991; Castles \& Miller 1993). This model stands for the strong French Republicanist tradition that dates back to the age of the French Revolution and Napoleon (Brubaker 1992). It also has distinct roots in the political field and in the scientific or intellectual field, both of which played an important role in reproducing the republican model (Favell 1998b; Schain 1999). However, France has also witnessed the succession of various problem frames, in research as well as in policy. In the early 1980 s, there was rather a multiculturalist framing of immigrant integration, characterised by cultural differentialism and a pragmatic politics of 'insertion'. Only in the late 1980s was the French Republican model revived in both politics and research.

\section{Politique d'insertion}

How have the French framed and reframed their approach to immigrant integration over the past decades? Until well in the 1970s, there was a belief in France that many immigrants would, in fact, return to their home countries (Weil 1991). Although this resembled the Dutch situation in that period, the difference was that in France immigration was already perceived as permanent. Following the immigration stop in 1974, after the oil crisis and the continuation of new immigration from former French colonies such as Algeria, the need for an integration policy appeared on the agenda in the early 1980s, several years after this had occurred in the Netherlands.

In the early 1980s, a model of incorporation was developed that had distinct multiculturalist traits. It proclaimed that immigrant ethnic minorities, or collectivities, had a right to be different - droit à la différence. This right involved a 'separatist demand for egalitarian treatment' (Feldblum 1999: 33), invoking politics to create equality for immigrant collectivities in various domains such as culture, religion, politics, labour, and welfare. It also led to differentialist policies, such as venues for immigrant collectivities to set up classes in their own language and culture so as to facilitate return migration rather than integration (Weil 1991; Brubaker 2003). These were mostly framed as specific elements of general state social policies (Favell 1998b). A sharp contrast to the republican model, this politique d'insertion (ibid.: 46-47) proclaimed the right to association, involving immigrant organisations in policy initiatives in diverse ways, such as in the establishment of a National Council of Immigrant Populations in 1984. The other side of the coin of insertion politics was a pluralist image of French society. President Giscard d'Estaing, and later also President 
Mitterrand, both declared that France had become a pluralist society (Feldblum 1999: 33).

Thus, when France first elaborated a model of immigrant incorporation based on the assumption that immigrants were permanent settlers, it deviated from the traditional French Republican model. It chose for a more pragmatic approach that often involved ethno-cultural accommodation and social-economic emancipation of these groups, rather than the typical republican assimilationism and individualism. In this sense, France was remarkably similar to the US and many other Western European countries mainly choosing for multiculturalist approaches at this time.

\section{Politique d'assimilation}

It was not until the second half of the 1980s that the traditional French Republican model was revived in relation to immigrant integration. According to Brubaker (1992: 49), the model was closely related to the founding myth of the French Republic; it was a cultural idiom that had powerful roots in French history. Reflected were various dimensions of the French Revolution: a bourgeois revolution that established a bourgeois society, a democratic revolution calling for active political citizenship, a national revolution based on civil equality for all French citizens and a bureaucratic revolution that established direct links between the citizen and the state.

The French Republican model valued individual citizenship. First, it was relatively inclusive, as citizenship was open to all those born on French territory - ius soli. This French conception of Staatsvolk majorly deviated from other nations, such as Germany, which defined its nation rather as a Volksstaat based on ethnicity, culture and descent - ius sanguinis (Brubaker 1992). Citoyenneté ('citizenship') thus unmistakable arose from the traditional French conception of a strong central state rather than of a French people, unlike Germany with its notion of the Volk.

Second, ethnicity, but also religion, culture and race, were considered irrelevant to the citizenship frame. The French model was 'colour-blind' (Weil 1991; Schain 1999). Instead of a right to be different, it contained a right to be indifferent - droit à l'indifférence - or the right to be treated as equals. Regardless of background, all people who are assimilated through French institutions such as education and the army (Schnapper 1991) become French citizens. This was closely related to the principle of laïcité, the secular nature of the French public space. Culture, religion and ethnicity belonged to the private sphere, whereas public areas were expected to be neutral and colour-blind. Officially recognising immigrant collectivities was therefore considered at odds with laïcité. In various creative ways, the government attempted to design policies so that they would affect the position of immigrants without implicitly recognising immigrant groups. For instance, the Fond d'Action Sociale pour les Travailleurs Immigrés et Leur 
Familles (FAS) was decentralised and connected to urban policy, so as to avoid state recognition of immigrant groups. This was a classic example of replacement strategies manifested in the French case. Recognising ethnic or cultural groups could effectively be avoided by targeting the neighbourhoods where ethnic and cultural groups were concentrated rather than directly targeting the groups themselves (De Zwart 2005). This approach contrasted dramatically with the general recognition of racial categories or ethnic and cultural groups as occurred in the Netherlands and in the US.

The French political tradition has always refused to recognise the American concept of 'ethnicity'. In the school, the factory, in the union [...] the ethnic dimension has never been taken into account, even if social practices don't always scrupulously follow this principle. It is not an accident that in France there have never been real ghettos of immigrant populations from the same country, on the model of Black, Italian or Hispanic neighbourhoods in the United States; that in poor areas immigrant populations from different countries mix with French people, apparently in the same social milieus. The promotion of Frenchmen of foreign origin comes about individually and not collectively through groups organised collectively. (Schnapper in Schain 1999: 200)

Finally, Brubaker (1992: 112) observes that there has always been some ambiguity to the nationalist traits of the French Republican model. The absence of an ethno-cultural conception of the French nation did not mean that there was no nationalism. In fact, nationalism manifested itself in the major emphasis being put on a need for assimilation. Immigrant communities were to be dissolved and integrated into the French Staatsvolk. However, this type of nationalism also occasionally led to more exclusionist policies. For instance, some politicians, particularly surrounding Le Pen, believed that specific non-Western migrants, such as Algerian Muslims, were 'unassimilable'. This exclusionist variant of nationalism became more prominent in the 1990s, when measures were implemented (such as the Pasqua Laws in 1993) that restricted access to French citizenship. Whereas citizenship was first considered an instrument for assimilation, in the 1990s the emergent approach stressed that migrants had to be willing to acquire French citizenship (volonté). This was a response to criticism from exclusionists that French citizenship had become desacralised 'être français, cela se mérite'.

\section{Beyond the republican model?}

Throughout the 1990s and after the turn of the millennium, the French Republican model became increasingly contested. Closely resembling the situation in the Netherlands during the same period, there were growing 
signs of disaccord between at least three frames of integration: assimilationism (the republican model), universalism (anti-discrimination policies) and multiculturalism (de facto multicultural practices on the local level).

First, the piecemeal development of a French anti-discrimination policy in the late 1990s involved the gradual emergence of a more universalist type of policy frame. A shift in focus from assimilation to anti-discrimination implied a shift in orientation, away from the rights and obligations of migrants to integrate towards the host society's responsibility to integrate migrants and combat discrimination (Fassin 1999; Geddes \& Guiraudon 1996). An anti-discrimination policy was proposed for the first time after the 1997 elections. This policy would generate a bipartisan support contrary to more affirmative action measures or multicultural approaches (Geddes \& Guiraudon 1996). Most notably, this anti-discrimination policy involved the collection of research on discrimination, the creation of a hotline to record discrimination complaints and the establishment of the advisory body Group d'Études et de Lutte contre les Discriminations (GELD). However, no independent body was created to handle discrimination complaints, as had been the case in the UK (Commission for Racial Equality) and the Netherlands (Commission for Equal Treatment). The development of this anti-discrimination policy was spurred on in important ways by the EU Race Directive that was passed in 2000 and had itself been inspired by the Dutch and UK approaches to anti-discrimination. The European Commission played a key role as a venue for promoting frameshifts beyond the realm of national politics and public debate, in this case obliging the French to adopt changes that would have been difficult to achieve via the national political arena.

The riots that occurred in the suburbs of major cities across France in 2004 proved to be a catalyst for the appearance of policies that involved a piecemeal de facto recognition of the ethnic, cultural and religious character of problems in these suburbs. The riots revealed the problems of French colour-blindness, which downright failed to address concrete social problems such as poverty, relative deprivation, spatial concentration, long-term unemployment and discrimination. A decoupling of the national discourse of the French model and the social reality and policy practices in French suburbs was becoming apparent. Favell (1998b: 184) described this as an 'increasingly sharp décalage between the highbrow republican rhetoric of the centre - the general symbolic issues involved in the definition of citoyenneté or laïcité - and the void of appropriate and consistent discourse and methods at the local level'.

This décalage was evident in, for instance, the rise of political mobilisation on an ethnic basis, which was distinctly at odds with the colour-blind republican model (Schain 1999: 210). Thus far, mobilisation of migrants had taken place mainly through institutions such as political parties, unions and organisations that were not ethnically exclusive, including France Plus 
and SOS Racisme. The ethnic mobilisation was partly a consequence of structural changes in the French political system, such as the changing positions of the French Communist Party (PCF) and the erosion of class solidarity in favour of ethnic solidarity (Schain 1995). Furthermore, ethnic mobilisation and heightened ethnic consciousness were also a consequence of state interference. As the government became more and more mired in migration-related urban problems, the pragmatic need for recognising organisations on an ethnic basis - that is, for ethnic categorisation - became obvious. To illustrate, one of the determining indicators for selecting Priority Urban Zones and Priority Educational Zones was that at least 30 per cent of the population in such an area be foreign. Another example of growing ethnic mobilisation and state interference was government's involvement in establishing a representative body of French Muslims, the Conseil de Réfléxion sur l'Islam en France (CORIF). Though largely unacknowledged in formal policy discourse, these measures do imply a real shift towards a more pragmatic approach at the level of policy practices.

At the same time, the French Republican model seems to be far removed from French political and academic discourse. In fact, after the turn of the millennium, there appears to have been a gradual 'return of assimilationism' in a way that resembles developments in many other European countries during this period (Joppke \& Morawska 2003). For instance, the French government announced an 'integration contract' for newcomers in France, which closely resembles the Dutch approach to the integration of newcomers. Moreover, in 2004, the High Council of Integration argued that government should break with 'the logic of guilt and discrimination' and return to the original idea of 'integration'. Clearly, far from being characterised by one dominant republican model, the French case at the beginning of the twenty-first century is one characterised by conflict between various frames, involving the traditional republican model as well as more multiculturalist and universalist frames.

\subsubsection{The construction of the French Republican model}

\section{The technocracy of insertion politics}

The preceding analysis of the French framing of immigrant integration shows that the republican model, for which France's integration policies has become internationally renowned, has not always dominated French policy. How can we explain these different frames, and to what extent have different structures of research-policy relations contributed to their rise?

The 'rediscovery' of the French Republican model seems to have taken place only at the point when migration and integration emerged, for the first time, at the centre of the political agenda in the mid-1980s (Favell 1998b; Feldblum 1999; Geddes \& Guiraudon 1996). Until then, technocratic efforts to avoid politicisation had kept both migration and integration 
policy firmly off the agenda. The administrative field dominated early policy developments in both domains (Geddes 2003). Administration enjoyed almost arbitrary power to regulate migration, through 'a maze of decrees, circulars, departmental memos or even telexes' (Wihtol de Wenden in Guiraudon 1998: 298). It was the legal field that intervened in this policy domain in the 1980s, through the venues of the Council of State and the French Constitutional Court. Under jurisprudential pressure from these legal bodies, the government was increasingly forced to formulate an official immigrant integration policy that accorded with the French law and Constitution, as well as with international law (Geddes \& Guiraudon 1996).

However, the politics of insertion in the domain of immigrant integration was mainly formulated in the administrative field; it was a product of 'technocratic elite management' (Favell 1998b: 47). According to Favell (ibid.), it was:

not really a grand idea at all, but a loose collection of narrowly targeted practices, which avoided treating the big symbolic questions that were to blow up centre-state in the 1980 s [...] by tracking and responding to the presence of new immigrants though a concern for their basic welfare and social needs and [...] by playing down the issue, away from the centre of party policies cleavages.

The politics of insertion was pragmatic and also systemically depoliticised. For instance, Feldblum (1998: 39) observes how Mitterrand and Giscard d'Estaing made an agreement in 1981 not to discuss immigration issues in electoral debates. Before the mid-1980s, when discussions about, for example, instant access to national citizenship emerged, they were often restricted to 'internal state discussions and revisions [...] defined as 'technical issues' and not political stakes' (ibid.: 59).

At this time, there was still hardly any involvement from the field of scientific research in immigrant integration. Moreover, little research appeared on this topic at all until the mid-1980s (Mayeur 2006). At that time, demographers, in particular, but also historians and sociologists, pioneered immigrant integration research in France (ibid.). One of the few celebrated studies from this period included a report commissioned by the Department of Culture by Giordan (1982), 'Cultural democracy and right to difference', which legitimised the multiculturalist policy discourse of the politics of insertion (Feldblum 1999).

\section{Issue linkage and politicisation}

A reconfiguration of the political as well as the scientific fields would be at the source of the reinvention of the French Republican model. The historical legacy of republicanism had remained a powerful cultural idiom in 
France even during the period of the politics of insertion. A politicisation of the political field and a dramatic alteration of the institutional logic of the scientific field and its boundaries with the political field led to the creation of a powerful cross-boundary discourse coalition in support of the republican model.

What is the explanation for the frameshift towards republicanism in the mid-1980s? In terms of structural developments in society, the French nation-state was pressured by several factors that seem to have been common to most European nation-states in recent decades (Favell 1998b). First, there was a revival of regionalism, which threatened to undermine the traditional, centralised Paris-centric conception of the French nation-state. Second, there was a decline of national solidarity or a sense of national belonging; social cohesion was also under pressure. Third, there were rising concerns about the scope of governance by central government and the feasibility of society, which coincided with particular concerns about the welfare state and the impact of Europeanisation and globalisation. These structural developments raised the government's sensitivity and sense of urgency concerning national identity and the functioning of the nation-state, thus paving the way for the revalorisation of national issues.

Immigration and integration fed precisely into this sort of sensibility. Within the political structure of competitive elites (Guiraudon 1998), they were prime issues for politicisation. This politicisation came from the political right. In the early 1980 s, the political right - in the form of the Rassemblement pour la République (RPR) and the Union pour la Démocratie Française (UDF) - was in disarray, a consequence of the dominance of socialist governance, and sought opportunities to revitalise its competition with the political left. In 1985, the UDF called for a reframing of the immigrant integration issue. This polarised the right and the left, with responses to the rightist discourse sometimes taking the form of accusations of racism (Feldblum 1999: 57). It was against the background of changing party strategies (left versus right) and state practices (the early 1980s' politics of insertion), that Le Pen and his National Front Party appeared on the French political stage (Schain 1988). Le Pen, however, radicalised the prevailing discourse, mixing its differentialist frame of immigrant integration - he saw Muslim migrants as essentially 'unassimilable' (see Wieviorka 1995) - with populist and regionalist opposition against the Paris centre held accountable for the malignant politique d'insertion.

With structural factors providing an opportune context for politicisation, the rise of Le Pen appears to have been an important situational factor (Brubaker 1992). Le Pen and the political right reframed immigrant integration as an issue of national identity, related to the meaning and value of what being French is - or what it should be. As such, the issue became linked to concerns about the nation-state. Honing in on citizenship, the right called for a more voluntarist conception of national membership, 
breaking with the republican model's automatic granting of citizenship to all those born on French soil (ius soli). This voluntarist reframing of French citizenship as something that had to be merited - 'être français, cela se mérite' - also involved communitarian arguments - 'France for the French' - and nativist arguments that questioned the loyalty of Muslim migrants, in particular (Feldblum 1999).

However, Le Pen's sudden advent and the radicalised discourse on migration and integration had adverse effects (Favell 1998b). First, the fierce accusations of racism made against Le Pen resulted in other parties, including those on the political right, becoming more reluctant to join forces with the National Front. Second, it created conditions for a left-right consensus on the revival of the traditional French Republican model of immigrant integration and national citizenship. This consensus was not only less differentialist than the politics of insertion but also less voluntarist, communitarianist and nativist than the ideas of Le Pen (Feldblum 1999). It led to a frameshift on the left, reframing integration as an issue of national identity, as well as on the right, abandoning its original call for more voluntarist, communitarianist and nativist citizenship.

Structural developments in French society appear to have contributed to the restructuring of the political field on migration and integration. This restructuring involved politicisation, left-right polarisation and the rise of Le Pen, and connected migration and integration to issues of national identity. Moreover, restructuring the political fields seems to explain the formation of a left-right consensus on the revival of the republican model of integration. The reframing of the republican model was not only highly contingent on politics, but also highly symbolic. However, a series of focus events in the late 1980s and early 1990s further reinforced the structural developments in this field,. The so-called Foulard Affair in 1989 that followed the headscarf controversy underscored the high principles of the French Republican model, such as citoyenneté and laïcité.

\section{Public intellectuals and the republican model}

Restructuring the political field and a revival of the French Republican model were also related to a restructuring the scientific field (Amiraux \& Simon 2006). France already had a tradition of major involvement by usually Parisian - intellectuals in public and political life (Bourdieu 1988). From the mid-1980s onwards, scientific and intellectual involvement in migration and integration would be dominated by specific intellectuals, often of a philosophical background. They contributed to 'public reflection of a highly philosophical nature, extraordinary in its abstract and theoretical content, and peculiar to the French political scene' (Favell 1998b: 40).

Highly politically oriented think tanks, such as the Club d'Horloge, which had links to the UDF and the National Front, and the Club 89, with links to the UDF and RPR, played an important role in legitimising rightist 
discourse on a revalorisation of French citizenship in the mid-1980s. Reports from these think tanks supported expansion of the discussion to eventually create conditions for reviving the republican model (Feldblum 1999: 38). Moreover, when the political field was successfully reframed in terms of national identity, leftist thinkers, including Alain Touraine, who was to become an influential intellectual in this field, also reframed their discourse in national terms. They shifted from a pluralist discourse to national, citizenship discourse (ibid.: 44).

In the 1980s, a broader group of intellectuals supported the renewed republican discourse, including, Dominique Schnapper, Pierre-André Taguieff, Alain Finkielkraut, Bernard Henri-Lévi and, somewhat later, Patrick Weil who was to become one of the most prominent republicanists to work on both sides of the research-policy nexus in the 1990s. They were public intellectuals in the sense that they derived their authority not only from their scientific field or their philosophical background, but also from their connections to politics and their reputation in the media. These public intellectuals further reinforced the focus of policy discourse on high republican principles instead of concrete urban problems in the 'banlieues'. Favell (1998b: 58) described them as:

a new generation of media-wise, self-promoting public intellectuals seeking to distinguish themselves from the dominant intellectual currents of the 1960s and 1970s, and keen to tender for direct political influence via all kinds of government-funded research projects, advisorships and commissions now available to the Socialists in power [...] as well as their fervent national republicanism, what characterised the more successful and mediatised figures - for example Luc Ferry, Alain Finkielkraut, Pierre-André Taguieff - was that they were almost always philosophers in tendency, not empirical social scientists; they frequently came from Jewish backgrounds; and they had apparently easy access to the most influential organs of the intellectual liberal press, Le Monde, Libération, Le Nouvel Observateur, L'Express and the widely-read academic journal Esprit.

Together with the left-right republican coalition in the political field, these individuals formed a discourse coalition that transgressed both sciencepolicy and disciplinary boundaries. It was impossible to distinguish their normative and factual claims, which fitted into the typical French tradition of normative social science. According to Favell (ibid.: 41):

This interdisciplinary, cross-party and cross-institutional consensus among ruling elites on the terms and language of the debate came together to marginalise thoroughly the claims of other possible ideas 
about the nature and future of French political unity: principally, the nationalist and culturally exclusive rhetoric of the far right, and the multicultural and internationalist ideas of the radical left.

This republicanist discourse coalition developed itself in opposition to another group of public intellectuals, with more poststructuralist and cultural relativist ideas, the so-called soixante-huitards, who counted Claude LéviStrauss among their number (ibid.: 58).

The public debate triggered by these intellectuals culminated in at least two government-established committees, whose reports would provide the basis for revival of the French Republican model. First established was the Nationality Committee, also known as the Wise Men Committee (Commission des Sages). This was set up in 1987 to reconsider French nationality law as a consequence of the politicised debates on migration, integration and national identity from preceding years. It was a product of the cross-party consensus developed at this time. The committee included mostly academics 'from elite Parisian backgrounds, in history, law, sociology and political science, including Alain Touraine and Dominique Schnapper' (ibid.: 42). While the committee only operated behind closed doors, it also held a range of public interviews with diverse actors involved in issues of integration and citizenship. The Nationality Committee was one of the first to formulate a republicanist approach to 'integration', a concept that initially surfaced in the period, being largely inspired by legal arguments, such as access to full citizenship, rather than by cultural arguments (Commission de la Nationalité 1988; Geddes \& Guiraudon 1996).

The Nationality Committee's work was succeeded by the establishment of the High Council for Integration (Haut Conseil a l'Intégration, HCI). HCI comprised civil servants and legal experts rather than academics (Favell 1998b: 42), and in 1993, provided an official definition of the republicanist approach to immigrant integration. It stressed the 'resemblances and convergences in the rights and obligations' of 'all those who are destined to live durably in our land' and emphasised the 'active participation' of all those in French society (see Geddes \& Guiraudon 1996). The HCI did not only advise government on reforms of the nationality law and integration policies, but also on the establishment of research programmes. It was to offer both scientific legitimacy and instrumental data for sustaining the republican model. According to Favell (2001):

the government appointed HCI [...] not only sought to formulate the normative, historical and political grounding for the new republican philosophy it espoused, they also set in motion a machine of empirical evidence gathering, explicitly constructed to find the data that the public theory had set out to prove. 
The formation of this discourse coalition in response to politicisation of the political field did not, however, produce greater responsiveness to the public. The French state's strongly centralised, unitary policy structure and the co-optation of French public intellectuals helped constrict debate to the national level (Guiraudon 2000b). Despite the debate's highly mediatised nature, the coalition and the republican discourse it sustained formed a new type of elite discourse (Schain 1995). The public intellectuals perceived their role as one of enlightenment, in which they had to defend and advocate the republican model at all costs. For instance, in the context of the Foulard Affair, they vigorously participated in public debate, defending French republicanism and warning that the affair should not become 'the Munich of republicanism' (Le Nouvel Observateur 2-8 November 1988 in Favell 1998b: 154).

\section{The Stasi Commission and the blind spot for empirical social science research}

A more recent episode illustrates the difficulties of achieving research-policy dialogues that reach beyond this French Republican model and the discourse coalition that supports it. In 2002 and 2003, a new series of Foulard Affairs occurred in France; the Islamic headscarf came to serve as a symbol of French integration problems, challenging the republican principle of laïcité. Consequently, President Chirac decided to establish a new commission to tame the episode of fresh controversies. The Independent Commission of Reflection on the Application of the Principle of Laïcité in the Republic, also known as the Stasi Commission after its chairman, Bernard Stasi, was given the broad task of reporting on issues concerning laïcité. Ultimately, this commission would recommend a ban on all 'ostensible religious signs', such as Islamic headscarves, in French public space. The commission comprised nineteen members, including intellectuals, administrators and politicians (Bowen 2007: 114).

What becomes clear from the commission's proceedings is that the focus gradually became the veil as a challenge to laïcité, rather than addressing the greater social aspects of integration and the place of religion in French society. According to Bowen (ibid.: 116):

the organization of the commission's work, the choice of people to hear and places to visit [...] all focused everyone's attention on this topic and on this hypothesis, rather than on the topic of laïcité, or on the topic of why it is that things are in such bad shape in these neighbourhoods.

Furthermore, while the commission included at least one empirical social scientist who was critical of the French Republicanist perspectives on integration and headscarf wearing, this member who was surrounded by a 
predominant republicanist mode of thinking eventually also voted for the joint recommendation of a legal ban, in order to preserve the unanimity of the committee (ibid.: 124). Moreover, among the many experts invited for interviews, no sociologists were asked to share empirical findings from their research concerning, for example, why Muslim women wear headscarves or social problems such as discrimination and poverty (ibid.: 19).

Like the Blok Committee in the Netherlands, which was established in roughly the same period, the Stasi Commission chose to remain within the frame of government policy, rather than critically reflect upon it and possible alternatives. This shows how the French Republican model continued to be reproduced by a discourse coalition of administrators, politicians and intellectuals.

Despite this blind spot for empirical social science, the 1990s in France saw a rise in more 'empirical' social research. This came in response to, among other things, mounting unease with the highly philosophical nature of intellectual and political discourses on immigrant integration. Bourdieu, for example, focused more on concrete issues in the banlieues, such as poverty, relative deprivation, criminality and anomie (Amiraux \& Simon 2006). Moreover, after the turn of the millennium, researchers would return to the dispute on statistiques ethniques (Sabbagh \& Peer 2008). It was claimed that the government would be unable to effectively implement EU directives on anti-discrimination if it was unable to keep track of direct and indirect discrimination of specific groups (Simon 2005). This shows how around 2000, the French field of immigrant integration research was becoming more and more fragmented. One of the vectors in this fragmentation appears to be the Europeanisation of immigrant integration research, a phenomenon also observed in the Dutch case. Interaction between scholars across Europe (and the US) generated more interest among French scholars in issues such as ethnic statistics, which is a cornerstone of immigrant policies in the UK, the US and the Netherlands. This Europeanisation - or internationalisation - also changed the French-American antagonism that had been a significant factor behind the development of the French Republican model.

\section{The ongoing boundary struggles on ethnic statistics}

Whereas immigrant integration research in many countries, including the UK and the Netherlands, was strongly influenced by the American sociology of race and urban sociology (promoted by the Chicago School and Robert Park), French discourse and research appears to have developed in a more antagonist relation to American research and discourse (Fassin 1999). Whereas American sociology framed immigrant integration as an issue of minorities and multiculturalism, French discourse stressed citizenship and colour-blindness. In French social research, America is often depicted as a counter model, in which the recognition of minorities has 
adverse effects such as ghettoisation - something avoided in the French Republican model (Bleich 2003). The American reference in French discourse often has the purpose of delineating the French model and warning against multiculturalism. As Fassin (ibid.: 226) put it: 'first, when discussing the politics of multiculturalism, "America" is the Other - a radically different culture of cultural difference [...] at the same time, the Other is a threat; we may not be so different any longer, we are indeed already engaged in a process of Americanization'.

This function of the American reference on how immigrant integration was framed in France relates specifically to a French taboo on social categorisation on the basis of ethnicity, culture or religion. Such classification is at odds with the colour-blindness of republicanism (Amiraux \& Simon 2006). For instance, data gathered by the official French Statistics Office (Institut National de la Statistique et des Études Économiques, INSEE) refer only to foreigners without French citizenship, as the categorisation of French citizens on their particular ethnic or religious origin is prohibited (Favell 2001). This means that second-generation migrants who would be categorised as members of minorities in, for instance, America and the Netherlands, remain invisible in French statistics. It also means that statements about policy success or failure are difficult to support with scientific data (ibid.).

The republican prohibition of ethnic categorisation significantly constrains empirical sociological research. This was illustrated by a controversial survey by social scientist Michèle Tribalat in 1995, who attempted to organise her data with questions referring to ethnic proximity and to French norms and values (Tribalat 1996). Conducted at the National Institute for Demographic Studies (Institut National des Études Démographiques, INED), this study broke the taboo on ethnic categorisation that was manifest in the official data from INSEE (Favell 2001). According to Favell (ibid.), the symbolic struggle over ethnic categorisation clearly illustrates how 'the criteria for competition in the intellectual field are not about empirical proof but about controlling the ideological high ground'.

In close correlation with the development of a (universalist) anti-discrimination policy around the turn of the millennium, the dispute on ethnic statistics was again revisited. In 1998, the High Council of Integration published a report for the first time on discrimination, and recommended the establishment of anti-discrimination group GELD. Various scholars, including demographer Patrick Simon, advocated the development of ethnic statistics vis-à-vis the 2000 EU Race Directive. This was not without effect, seeing as the Haute Autorité de Lutte contre les Discriminations et pour l'Egalité (HALDE) was established in 2005. Clearly, the development of the new anti-discrimination frame placed 'a normative issue requiring political and legal decisions and discussed either in juridical terms by 
conseillers d'État or philosophical ones by public intellectuals', as Guiraudon and Geddes (1996) into the hands of 'social scientists and 'social partners'. More than ever before, this put empirically oriented social scientists at the heart of the French debate. However, regarding a series of meetings on ethnic statistics in France and the US in 2006, Sabbagh and Peer (2008: 2) had to conclude that:

While there was general agreement among the American participants about the necessity and value of using statistical data to measure discrimination based on race and ethnicity, as well as to assess the impact of antidiscrimination policies, only a minority of the French participants shared this opinion.

This was confirmed by a 2007 petition in the French media signed by leading national academics, including republicanist Patrick Weil. The petition argued against any research that involved a categorisation on the basis of origin (see Guiraudon \& Geddes 1996). This makes it obvious how in both policy and academic discourse, the republican model was far from dead, and the dilemma of ethnic statistics is as much a controversy in France's academia as its politics.

\subsubsection{French Republicanism across research-policy boundaries}

The French case contains vivid examples of how a changing division of labour between science and politics contributed to reframing the immigrant integration issue. Once France came to terms with not only having become a country of temporary migration, but also admitting that immigrants were now permanent settlers, the issue was reframed in a distinctly technocratic way. The administrative field had a heavy hand in framing the policy as a kind of 'technocratic elite management', in which central and local governments were majorly implicated. At the same time, politicisation was systematically avoided. Framing responded to pragmatic needs from central and local administration, rather than deliberations on symbolic issues or matters of principle. The legal field was crucial in forcing government to stop ruling by discretion and to adopt formal policies. This stood in contrast to the marginal role given social scientists. On the few occasions social scientists were involved, notably as demographers and sociologists, they legitimised the multiculturalist, pluralist policy discourse that had been generated in the administrative and legal fields. The legal field advocated recognition of difference - unlike the traditional republican model because of national and international principles of law such as equality; the administrative field preferred a degree of cultural pluralism, based on a belief that it would be most efficient in terms of the pragmatic resolution of 
concrete, local issues of immigrant integration. It was this technocratic division of labour that sustained the politique d'insertion of the early 1980s.

Changes in the structure of the political and scientific fields in the midto late 1980s contributed to a frameshift that revived the traditional French Republican model of integration and citizenship. This interaction appears to have been generated, in particular, by an elite discourse coalition advocating French Republicanism across the boundaries of both science and politics. In the political field, a number of think tanks associated to political parties on the right punctuated the norm of depoliticisation that had sustained the politique d'insertion, and called for a more assimilationist policy approach. This compelled a political reconfiguration - across the left and the right - of immigrant integration in terms of national citizenship. In other words, politicisation and Le Pen caused a revival of the French Republican model in the political establishment. Meanwhile, in the scientific field, policy-oriented social scientists were gaining influence. A number of public intellectuals, often with a philosophical background, would play an important role in advocating and defending the French Republican model.

The politique d'insertion frame was largely pragmatic and technical. Now, the discourse coalition of scientific public intellectuals and the republican coalition between the political left and right reframed immigrant integration as a symbol of larger societal issues such as national identity, the emergence of Europe and the viability of the national welfare state. The new division of labour between science and politics, triggered by politicisation and issue linkage, contributed to the revival of the French Republican Model. The administrative (local governments) and legal fields (Council of State, Constitutional Court) could only defend certain aspects of cultural pluralism and achieve some depoliticisation, albeit in an inconsistent, touch-and-go manner. However, they could never really undermine the revival of the French Republican model. The scientific-political discourse coalition for republicanism defined its role in terms of enlightenment - defending the high principles of the universal French model - as illustrated by its response to the Foulard Affairs. As such, the politique d'assimilation was a project of the French elite.

However, this discourse of republican enlightenment appears to have generated specific pathologies that would gradually trigger another change to the division of labour and problem framing. There was a spreading décalage between the national republican rhetoric and the de facto multiculturalism found at the local level. In response to concrete, problemexerted pressure at the local level, governments adopted a more pragmatic approach to coping with these difficulties. On many occasions (such as the establishment of FAS and CORIF), this led to measures at odds with the national republican model. In the political field, growing problem-exerted pressure from the banlieues also sparked greater support for an approach 
to integration that would target more specific problems, rather than philosophical matters or symbolic issues such as national identity. In the scientific field, more empirical social research seems to have emerged on issues related to migration and diversity in French society

\subsection{Germany}

\subsubsection{A reluctant country of immigration}

Germany was one of the last Western European countries to formulate immigration and immigrant integration policies. This was due to a belief - or a norm - that Germany was not and should not be a country of immigration: Kein Einwanderungsland (Faist 1994). Although there are notable differences across the various German Länder, in terms of their regional, more pragmatic and ad hoc policy responses to immigration, this norm has proved much more resilient in Germany than in many other European countries, including the Netherlands. Somewhat paradoxically, despite the absence of integration policies, Germany has become internationally renowned for the extent to which it has granted legal and social rights to immigrants, although largely excluding them from political rights (Guiraudon 1998; Soysal 1994). It has been considered an example of post-national immigrant membership, which means that citizenship involves universal personhood, rather than membership of a specific nation. The following section will explore the development of this model and the more recent turn towards assimilationism that seems to be occurring in Germany as well as a series of other European countries (Brubaker 2003; Joppke 2003).

\section{A differentialist frame: Kein Einwanderungsland}

Germany held on to the belief that it was not a country of immigration much longer than most of its surrounding countries. More than just a belief, not being an Einwanderungsland had a distinct normative character, tied up with German's history, culture and nation-building experience (Joppke 1999b: 62). Whereas in France the nation and the state were closely bound in the French notion of Staatsvolk and ius soli (citizenship as birthright), the German nation-building legacy retained a distinct discrepancy between nation and state (Brubaker 1992: 137). The German nation was defined on ethno-cultural ties, as a Volksgemeinschaft or a community of common descent, characterised by ius sanguinis (citizenship as a right of blood) instead of ius soli. This way of constructing the imagined German Volksgemeinschaft dated from the Wilhelmine period, in which German (Prussian) territory did not fully overlap with what was conceived to be the German nation; there was a large German diaspora throughout Eastern Europe and there was also a significant Eastern European 
immigrant population (e.g. the Polish) within Germany (ibid.). This made Germany a 'nation without a state' or an 'organic Volksgemeinschaft' emphasising the exclusion of Fremdvölkisch ('foreign people') and, in the Nazi period, the exclusion of Jews, in particular (ibid.: 166).

This ethno-cultural nation-building legacy was revived after World War II, in the context of the separation of East and West Germany. The belief in an organic German Volksgemeinschaft provided the basis for defining Germany as an incomplete nation so long as East and West were separated (Geddes 2003). This was one of the reasons for Germany's reluctance to concede to being an immigration country: being an immigration country was at odds with the organic conception of the Volkskgemeinschaft that fed the national longing for reunification. As a consequence, naturalisation was tremendously difficult for immigrants, as it would require 'a social transubstantiation that immigrants have difficulty imagining, let alone desiring' (Brubaker 1992: 78). In the 1980s, the annual nationalisation rate among immigrants was as low as 0.5 per cent and dual nationality was ruled out as a consequence of German constitutional thought, which had evolved since the Wilhelmine period (Geddes 2003: 94). Although the naturalisation procedure was relaxed in the $1990 \mathrm{~s}$, the annual naturalisation rate rose only to about 1 per cent in 1995 and dual nationality remained a controversial topic in Germany.

The denial of being an immigration country was distinctly at odds with the post-war reality in which Germany witnessed very significant and diverse immigration (Joppke 1999a). First, Germany experienced the arrival of large numbers of foreign workers, Gastarbeiter, mainly from Turkey and the former Yugoslavia. Moreover, in line with its self-definition as a Volksgemeinschaft, Germany orchestrated one of the largest state-led immigration projects ever, to repatriate Germans, the so-called Aussiedler, from its diaspora, particularly from Eastern Europe and the former Soviet Union (Thränhardt 2000). The Aussiedler enjoyed a constitutional right to return to Germany. Finally, Germany has been a main receiving country for large numbers of asylum seekers; its Constitution protects a basic right for applicants to file an asylum application in Germany, without allowing the German state to limit migration to its territory. This came in contrast to ideas that international human rights regimes and discourse would constrain national sovereignty; rather, it was a case of self-constrained sovereignty, connected to German history (Joppke 1999b).

In this context, Germany has been reluctant to develop an immigrant integration policy, as this would be self-acknowledgement of being a country of immigration. Instead, it promoted programmes that would facilitate return migration, orientating migrants towards their countries of origin rather than the host society. Those measures taken in the domains of labour and education were considered for 'temporary integration' (Joppke in Broeders 2001: 71). Furthermore, they often involved more pragmatic, ad hoc policy 
responses at the local or regional levels. This led to an ambivalent approach, not unlike the two-tracks policies adopted in the Netherlands in the 1970s. Until as late as 1997, return migration would remain an important policy aim, as demonstrated by the policy memorandum 'Integration, restricting immigration, help with voluntary return migration'1 (ibid.: 76).

\section{Institutionalised separateness and the welfare state frame of integration}

Parallel to this persistent denial of being a country of immigration, immigrants were nevertheless granted significant legal and social rights. In this way, Germany gradually established a very particular type of multiculturalism (Joppke 1999b: 191). First, immigrants became post-national members of state. These 'denizens' (Hammar 1985) enjoyed equality in the legal and social-economic sphere, with equal rights as national citizens, while being excluded from political rights or membership of the German nation. Post-national membership fitted with the divergence of state and nation in German history and culture. It made Germany an interesting case study for post-nationalist thinkers (Soysal 1994), although this 'post-nationalism' had national, rather than international or global, roots (Joppke 1999b). In this respect, German policies towards immigrants were not geared towards a socially constructed category of ethnic or cultural minorities but rather, what were considered national minorities - or minorities belonging to a particular nation who remained oriented towards that nation. This explains why Germany did not experience a rise in ethnic politics as the Netherlands and the UK did; immigrants, such as Turks, remained loyally oriented towards their homeland politics (ibid.) - a form of 'transplanted home politics' (Koopmans \& Statham 2003).

This post-national framing of immigrant 'denizenship' by the German state coincided with a sharp focus on the role of the welfare state in relation to immigrant integration (Bommes \& Geddes 2000). Bommes (2000) describes this as a 'community of GNP contributors' comparable to the German 'community of descent'. Neither nationality nor ethno-cultural origin mattered in the German model of integration but rather, territory and an individual's participation in the social-economic sphere of the welfare state (Thränhardt 2000). This frame of integration was therefore colourblind, albeit grounded within a very different frame than in France.

Second, in social-cultural terms, this approach also involved a sort of institutionalised separateness, or ethnic separatism (Brubaker 2003; Castles \& Miller 1993; Green 2004). In various domains, while in some regions more than others, facilities such as immigrant minority language classes were established as a means to accommodate cultural pluralism, such as in the sphere of welfare policies. However, in contrast to France and the Netherlands where ethnic accommodation was part of a multiculturalist policy of emancipation, in Germany it was considered part of 'foreigners policy'. 
policies in these three domains - schools, social services and citizenship - and the idioms in which they were rationalized and justified were indicative of a kind of benevolent, paternalistic, and egalitarian (or pseudo-egalitarian) apartheid, an institutionalized separateness, suggested in the oxymoronic phrase 'unsere ausländischen Mitbürger' - our foreign fellow citizens - that has been a leitmotif of well-meaning public discussion of what continues to be called Ausländerpolitik - politics or policies regarding foreigners - in Germany. (Brubaker 2003: 46)

\section{An assimilationist turn?}

A frameshift has been taking place in Germany over the past decade or so, putting increasing pressure on the post-national problem frame and gradually shifting towards a more assimilationist frame. First, when East Germany and West Germany were united in 1990, the incomplete nation argument dissipated and, as it did, the powerful political-cultural norm of not being a country of immigration also came under fire. Second, Germany witnessed massive asylum migration in the early 1990s, which forced the government to adopt a so-called 'asylum compromise' that allowed more discretion in restricting immigration while, at the same time, promising to make its citizenship regime more accessible to foreigners. This led to a normalisation of immigration during the 1990s, adopting restrictive migration policies towards foreign workers and asylum seekers, as well as Aussiedler (Geddes 2003). Third, the effects of the German post-national approach on the position of migrants appears to have been detrimental; increasingly, the post-national model was considered a failure (Hansen 2003). For instance, in terms of naturalisation, employment and language acquisition the position of migrants remained marginal (ibid.). Finally, there were rising concerns about racism and discrimination in the early 1990s. This was a consequence of several highly mediatised focus events, such as 1993's incident in Solingen where extreme-right youth burned the home of a Turkish family, resulting in five deaths.

These events gradually pushed the German approach towards a civic nation à la française, with a more assimilationist approach (Joppke 2003; Joppke \& Morawska 2004). As the citizenship regime was relaxed, some ius soli (birthright) elements were introduced in order to make naturalisation easier and citizenship more accessible. Until this point, cultural assimilation had been a condition for naturalisation and the German state enjoyed great discretion in granting it to newcomers. Now, national citizenship became attainable, although still subject to strict conditions. This meant that naturalisation was gradually reframed as a means for integration, rather than as an outcome of integration or assimilation (Hansen 2003: 95). However, dual nationality remained an obstacle; immigrants still had to 
select either their German or original nationality at the age of maturity. As a consequence, even in the 1990s, the annual rate of naturalisation hardly rose (Geddes 2003). As a result, the effects of the assimilationist turn have not yet been very significant; however, at least in the policy discourse, there has been a turn towards 'politically recognizing, legally constituting, and symbolically emphasizing commonality rather than difference' (Brubaker 2003: 47). This coincided with emerging discussions about the German Leitkultur ('guiding culture') in national political and public debates (Geddes 2003).

After the turn of the millennium, following a report from the Süssmuth Committee, the government finally acknowledged that Germany was in fact an Einwanderungsland (Hansen 2003). This led not only to greater efforts in immigration policy, but also endeavours to formulate a more substantial integration policy; to do this, Germany often looked to its neighbours across the border, the Netherlands (Geddes 2003).

\subsubsection{Germany's 'muddling-through' integration}

\section{Muddling-through policymaking}

How can Germany's particular framing of immigrant integration and the frameshift that has taken place be explained? Firstly, it is important to observe that the German policy of social integration was not so much the product of a grand central policy design, such as the French Republican model or the Dutch minorities policy. Rather, it was the consequence of an institutional process of 'muddling-through' by Germany's local, regional and national administrations, politics, social scientists and legal venues (Joppke 1999b: 187; Schneider 2007). This muddling-through involved, on one hand, coping with the negative feedback that sustained the denial of being a country of immigration, for a long time based on the charged argument of an incomplete German nation. On the other hand, it involved coping with the permanent settlement of rising numbers of migrants. This put increasing pressure on the policy monopoly of not being an immigration country, to formulate immigration policies and to adopt measures to ameliorate the social, economic, cultural and political position of migrants. Bommes (2004: 161) speaks, in this regard, of the hypocrisy of German policies, noting how 'the political front stage was dominated [...] by the official mantra that Germany is no immigration country', while 'backstage, the legal obligation to include migrants [...] in the general welfare provisions was more and more transformed [...] into an everyday routine'.

Lack of a grand central policy design was reflected in the absence of a unitary, central policy structure. The federal structure of the German state meant that local (Länder) and regional governments (Bundesstaten) enjoyed significant autonomy in several key domains of integration, such as education, welfare and culture (Soysal 1994: 62), also setting up local and 
state offices that specialised in integration policies and creating networks for consultation with immigrant self-organisations (ibid.: 78). This led to significant differences between regions. For a long time, some states, such as Bavaria, maintained immigrant minority language courses in order to facilitate return migration; in some states these courses were aimed at accommodating ethno-cultural diversity, while in others they were abandoned altogether, being declared an obstacle to German language apprehension (Hansen 2003). This autonomy of local and regional governments created a large number of venues where new policy ideas could emerge. For instance, in the late $1980 \mathrm{~s}$, several states decided to afford voting rights to immigrants that had been resident for a specific period (Joppke 1999b). Although their decision was eventually annulled by the German Constitutional Court, which interpreted the Constitution in such way that the right to vote was to be confined to the German people only, it does show how local and regional venues do furnish opportunities for positive feedback towards policy change.

These venues in the German federal system had an important part in the piecemeal attribution of legal and social rights to migrants. On the national level, there was 'no unitary structure or co-ordinating unit' for integration or immigration policies (Guiraudon 1998: 296). There was a Federal Commissioner for Foreigners, the Ausländerbeauftragte (within the Department of Labour and Social Affairs), which has 'when similar agencies in other countries are considered [...] a much weaker position vis-à-vis both the state and migrant groups' and significantly lacked 'resources and attention from government' (Soysal 1994: 78). Moreover, the role of this commissioner and committee within the department was deliberately kept small-scale, as part of a political strategy to avoid any suggestion that Germany had become a country of immigration (Guiraudon 2000b).

At the same time, this created tension between the autonomy that was granted to local and regional governments - who often used this power to extend immigrant rights and accommodate pluralism - and central government, notably the Department of Home Affairs (Joppke 1999b: 192). Embedding the integration policy in the Department of Social Affairs and the Auslanderbeauftragte was therefore an important indication of framing immigrant integration in terms of social-economic participation and the welfare state, rather than in terms of home affairs and national citizenship, as advocated by the Department of Home Affairs.

National government involvement in this policy domain was mainly through semi-public organisations that were organised at the national level and associated with government - what Soysal (1994: 77) describes as a 'statist-corporatist' pattern of incorporation. This included a series of welfare organisations providing social assistance to migrants, such as Catholic organisations that targeted mainly Catholic migrants, Protestant organisations for Protestant migrants and welfare organisations often associated 
with the Social Democratic Party that catered to the large remaining group, notably including Muslim migrants such as Turks. The organisations were represented in a Department of Social Affairs committee for the coordination of the integration policy, which also involved various other governmental and public organisations, federations of trade unions and business organisations and church organisations (ibid.: 78) In contrast to the UK and the Netherlands, migrant organisations themselves were not present in such a central government committee (ibid.).

\section{Correspondence between immigrant integration research and policies}

Social scientists played an important role in the 'muddling-through' approach to immigrant integration. They legitimised the welfare state approach that emerged within the setting of the broader German differentialist frame of being Kein Einwanderungsland. Social researchers have generally zeroed in on 'social economic factors' and 'mainly on the welfare state context of immigrant inclusion' (Lavenex 2005: 250). According to Lavenex (ibid.), 'the successful integration of non-nationals in Germany is discussed as a function of their participation in the social and economic institutions of the welfare state, including in particular schools, professional training and the labour market'.

Bommes (2004: 197) also speaks of the pronounced 'embeddedness' of German research and its approaches within 'the model of the German welfare state'. Especially since immigration levels surged due to labour migration, German scholars, particularly sociologists, became highly preoccupied with the social integration of migrants into the German welfare state. In this respect, German immigrant integration research developed within the political frame of reference that emerged in the 1970s and 1980s; 'the primacy of problems was defined in politically rather than scientifically embedded frames' (ibid.: 199). Bommes (ibid.: 198) says that this 'striking homology between the perspectives of the social sciences and the Sozialstaat' is due to a powerful process of co-evolution of immigrant integration research and policy in Germany, or what he describes as:

a relation of correspondence and mutual support between science and politics: the orientation of research to the public and political definitions of migration and integration and the effective message that migration and integration constitute important issues of the present and future, succeeded in creating political resonance and a corresponding willingness to provide the means required for research.

According to Lavenex (2005: 251), the paradigm of welfare state integration had already been present in the pioneering stage of German immigrant integration research. For instance, Hoffman-Nowotny (1973) adopted a macro-sociological approach on migrant inclusion and exclusion in terms 
of opportunities to participate in various systems, such as labour, education and politics, without focusing on the cultural domain. In a similar vein, Esser (1980) defined migration and integration as a function of compatibilities between the rational interests of migrants and societal systems. The welfare state frame of immigrant integration also clicked with the German ethno-cultural frame of national citizenship, that a priori excluded the option of assimilation, which could only mean being born as a German (Lavenex 2005: 257). In addition, it was in line with German discourse about the success of its welfare state, with American laissez-faire liberalism as its counter image. Finally, the welfare state frame and post-nationalism did not violate the taboo on critically assessing religious, cultural and ethnic differences (ibid.). Instead, it provided a powerful counterargument to racist discourse.

In contrast to this integration paradigm, 'the ethnic dimension of integration is rarely stressed' in German immigrant integration research (ibid.: 250). Lavenex (ibid.: 252-253) quotes Faist (1994), among others, stating that disagreements about ethnicity and multiculturalism were deliberately avoided, as they would lead to ideological struggles. Unlike countries such as the UK, the US and the Netherlands, minority approaches never really gained ground in Germany. These approaches were immediately criticised, by those supporting the dominant German frame of welfare state integration, as 'creating the societal construction of immigrants as a social problem' and 'ignoring the main underlying socio-structural conditions for the emergence of the immigration situation since the 1980s' (Bommes 2004: 177-178).

Studies that did raise ideas countering German post-nationalism and its welfare state integration frame risked being ignored. For instance, as early as 1979, Germany's first Federal Commissioner for Foreigners (Ausländerbeauftragte), Kühn, had issued a report that demanded fundamental reform of the German citizenship law, including extending voting rights to migrants (Guiraudon 2000b). Such ideas resembled those put forward in the WRR report 'Ethnic minorities' in the Netherlands from that same year (Thränhardt 2000). However, Kühn's report remained largely ignored, especially when it came to more fundamental recommendations such as voting rights. It was then still too controversial for the government, given the German policy context and the persistent ethno-cultural selfunderstanding, to adopt such a radically new approach.

This type of research-policy nexus seems to approximate the engineering model; research is highly policy-oriented but operates in a setting characterised by political rather than scientific primacy and usually contributes to legitimating a specific mode of government discourse. In this case, German social researchers were undoubtedly pivotal in engineering the German (universalist) welfare state frame of integration. The Dutch integration policy of the 1990s closely resembled this German welfare state 
frame of integration. However, an important difference is that, in Germany, the welfare state had been the dominant frame for the evolution of policy as well as research in a relatively uncontested way; in the Netherlands, the rise of universalism in the late 1980s was accompanied by major frame conflicts and boundary struggles.

However, since the 1990s, German immigrant integration research has become more differentiated and generalised (Bommes 2004). Its closer connection to general social science disciplines - particularly, general sociology - has diluted what was once a remarkable emphasis on welfare state integration (ibid.). At the same time, another vector at work is the Europeanisation of immigrant integration research. As also observed for the Dutch and French cases, German migration scholars are interacting more and more within international research networks. This has added further to differentiation in terms of research approaches and knowledge paradigms, notably contributing to the rise of transnationalist frames in German immigrant integration research (in a way that is very similar to the Dutch case). Transnationalism seems, however, highly compatible with the traditional German welfare state approach to integration, as illustrated aptly in the work of German sociologist Thomas Faist (2000a, 2000b). Whereas multiculturalism and assimilationism remain within the boundaries of the nation-state, transnationalism loosens this grip. It highlights not how immigrants are incorporated into the nation-state but instead, how migration can bring about integration in various social spaces. The nation-state is just one of those social spaces; others include the welfare state or an international human rights regime.

\section{Politicisation and consensus-seeking: The Independent Commission on Immigration}

Compared with the UK and the Netherlands, immigrant integration was politicised relatively early in Germany (Guiraudon 2000b: 145). Whereas there had been a broad consensus on immigration until the 1980 s, by the end of the decade, political party competition surrounded the issues of migration and integration (Thränhardt 2000). With anti-communism sentiments waning, the Ausländer issue became a source of party conflict, especially for the centre-right CSU/CDU union (ibid.: 165). In the early 1990s, anti-immigrant parties such as the National Democrats (NDP) and the German People's Party (DVU) also made the CSU/CDU more eager to take a tough stance on migration and diversity (Geddes 2003). Moreover, by the end of the 1990s, fierce competition erupted between the SPD/ Green coalition in government and the CSU/CDU in opposition, concerning plans to introduce more ius soli elements to the law on German citizenship. The opposition arranged a broadly supported petition in which it called 'For integration - but against dual nationality' (ibid.: 96). In 1999, having suffered a major defeat in elections in one of the Länder, the 
government coalition had to give way on a number of major points. That immigrants still had to choose between their German or original nationality at maturity continued to form a significant obstacle to naturalisation.

As Guiraudon argues (2000: 137), this politicisation may be explained by the absence of a strong central policy structure and the abundance of local, regional and federal venues. The same argument is made by Green (2004: 139), who sees the lack of a clear institutional policy structure as one of the reasons this area could be prone to such heavy politicisation and polarisation. Several other factors explain why this politicisation was milder than observed elsewhere, for instance, in France. First, racism in Germany has been a huge taboo, a legacy of its Nazi history (Thränhardt 2000). As concerns about racism rose in the early 1990s, with the emergence of anti-immigrant parties and a series of focus events involving racism, such as Solingen in 1993, the government faced mounting pressure to create a new elite political consensus on this issue. This contributed to the development of the 1993 asylum compromise (Joppke 1999b).

Second, Germany has been characterised by a culture of consensus-seeking, Verhandlungsdemokratie, not unlike that in the Netherlands (Guiraudon 1998: 296). Because of the multiparty system, there is no 'winner-takes-all logic' that might contribute to politicisation but rather, political parties are forced to rule alongside each other, with the liberal democratic FDP party in a particularly pivotal role. An important facet of this German Verhandlungsdemokratie has been, as Schneider (2007) shows, the establishment of 'independent commissions' that helped create inter-party consensus on complex, contested policy issues. Often, these commissions were 'independent' in that they involved representatives from various parties and incorporated the expertise of academics and interest groups.

In the field of migration, too, an effort was made to tame the ongoing politicisation through independent expertise. Major party conflict emerged when, at the end of the 1990s, the Schröder (SPD) government decided to allow selective labour migration, especially in highly skilled areas such as IT. Faced with political opposition from the CDU, who maintained that Germany should not be an Einwanderungsland, then Minister of Interior Schilly decided in 2000 to establish an Independent Commission on Migration to Germany (ICM). This commission was to develop a comprehensive outlook on the implications of migration, asylum and integration. Although only a relatively small number of its members were academics, the credentials of its chair, Rita Süssmuth, gave important legitimisation for the commission's work (ibid.: 102). However, the main function of this commission would not be to 'enlighten' politics with a new frame but rather to create consensus and avoid party political conflict (ibid.: 103). The fact that Süssmuth was a prominent member of the CDU and former chair of the German Bundestag added to the authority of this consensusbuilding effort. According to Boswell (2009), the relatively weak role of 
academic expertise in this commission can be explained by the government's efforts to avoid being labelled as technocratic and out of touch with the public.

The Süssmuth Commission tried to impose a more rational tone on the immigrant integration debate. It recommended a more rational view be developed concerning the economic benefits of selective labour migration and, emulating the Dutch example, the necessity of developing civic integration courses for newcomers (Schneider 2007). It also suggested the need for a more systematic provision of data and analysis on which German migration policies should be founded (Boswell 2009: 151). Furthermore, one of the background papers to the Süssmuth report recommended establishing a new research institute affiliated with the government agencies responsible for such data and analyses.

However, as was the case in the Netherlands with the Blok Committee, Germany's effort to tame the ongoing political controversies with independent expertise would prove rather ineffective. In the midst of proceedings, the CDU, angered that Süssmuth had agreed to head Schilly's commission, established its own commission on immigration. Both commissions' reports were published shortly after one another, in June and July 2001. Their timing would prove unfortunate in view of $9 / 11$, as well as early 2002's appearance of CSU leader Edmund Stoiber on the German political stage. Stoiber advocated a much more conservative tone vis-à-vis migration and integration (Green 2004: 123-124). Furthermore, the Süssmuth Commission was accused of having an elitist, technocratic style that was entirely out of touch with public concerns about restricting immigration and furthering immigrant integration (Boswell 2009: 103). Boswell connects this wariness of technocraticism to the legacy of the guest worker era, which would also have been driven by technocratic views on migration rather than by consideration for migration's impact on society.

Controversies surrounding the Süssmuth Commission were clearly related to the underlying frame conflicts in Germany. The commission's rational social-economic emphasis was simply at odds with the much more social-cultural frame of the CDU/CSU, who articulated public concerns about the societal impact of migration and the preservation of the German Leitkultur (Boswell 2009; Green 2004). Rather than compelling a process of critical reflection on these different frames, the commission (like the Blok Committee in the Netherlands and the Stasi Commission in France) chose a specific frame, thereby dissociating itself from the multiplicity of frames present in both political and scientific discourses. The new migration law that had been recommended by the Süssmuth Commission was ratified only years later through difficult negotiations and compromises reached between the main political parties, though only after it was first rejected by the German Constitutional Court in 2003. 
The evolution of a bureaucratic nexus

The Süssmuth Commission did create a new discourse that embraced Germany's becoming an Einwanderungsland and its need for a more systematic policy of immigrant integration (Schneider 2007). In this context, it had also created awareness that policymaking should be sustained by more systematic research and data (Boswell 2009). Whereas Germany had never developed an institutionalised research-policy nexus like the Netherlands or the UK (Schneider 2007), this new consciousness did create a very different pattern of boundary work in Germany. Following the Süssmuth Commission, an Immigration Council was established in 2003, which was to advise on the (then still to be ratified) new immigration law (Boswell 2009: 151). This council was again chaired by Süssmuth, and its members comprised several academics. However, due to difficulties in getting the law ratified, the council was disbanded after a year. As Boswell shows, though, the council further reinforced the need for a more systemic approach to data and research (ibid.: 152).

This led to 2004's establishment of an in-house research group within the German Federal Office for Migration and Refugees (Bundesamtes für Migration und Flüchtlinge, BAMF). It took over the main functions of the Immigration Council. However, as Boswell (2009) shows in her in-depth analysis of the research group, its main function was to bolster BAMF's organisational legitimacy in relation to other organisations who claimed authority in Germany's emerging field of migration policies. Boswell found very little evidence of instrumental or substantive uses of the research by the group. There was 'a strong respect for knowledge as a source of organizational legitimacy, but limited interest in drawing on research to justify policy preferences' (ibid.: 171). Rather, what the research group mainly provided was general background information and data.

This way of configuring the first institutional research-policy nexus in Germany reflects, at least to some extent, the rational bureaucratic mode of governance that applies more broadly in Germany. On one hand, this involves clear-cut boundaries: the role of research is clearly demarcated from politics and independent expertise is highly valued in the political arena. On the other hand, it involves prominent political primacy: the role of research is to help politics rather than to do politics. The institutionalisation of a migration policy domain, now that Germany had recognised it was an Einwanderungsland, also brought about a degree of isomorphism in aligning research-policy relations in this field with the more general bureaucratic pattern in Germany. 


\subsubsection{The co-evolution of German immigrant integration research and policies}

The German approach to immigrant integration is often depicted in migration literature according to the 'differentialist model'. However, as an analysis of the development of immigrant integration research and policy in Germany has shown, this model was heavily contested over the past decades, thereby needing to be supplemented by several alternative frames. First, there was a strong welfare state frame of immigrant integration among researchers and policymakers. Rather than integration into German national society, which was excluded by the ethno-cultural conception of 'Germanhood', researchers and policymakers were mainly concerned with integration in the German welfare state (Lavenex 2004). This welfare state approach to integration has also been qualified as post-national, endeavouring to include migrants in specific institutions rather than to assimilate them into a specific national community. However, it also carries distinct characteristics of a universalist frame, the kind that had emerged in the Netherlands during the 1990s. Furthermore, an assimilationist frame focused on the German Leitkultur in public debate and the political arena, although it has hardly yet been articulated in the field of German immigrant integration research. This shows how the German situation, contrary to Brubaker's institutionalist claim, must be qualified as a multiplicity of frames rather than a dominant national model. As Green (2004) has argued, there has been an almost continuous conflict between different frames of immigrant integration in the political arena, which explains the slow and incremental development of German policies, rather than the resilience of the German differentialist model.

A clear pattern of co-evolution in the development of German immigrant integration research and migration policies is evident. At least until recently, this co-evolution was not triggered by an institutionalised researchpolicy nexus, as was the case in the Netherlands. There was, though, a clear relation of 'mutual correspondence' between the framing of immigrant integration in research and policy. This relation of correspondence evolved around a frame of welfare state integration. However, researchers' involvement in policymaking was never very direct. Rather, immigrant integration research operated in a setting in which the framing of immigrant integration was set primarily in the political arena. This was similar to France, where immigrant integration was politicised relatively early. As such, the German nexus between research and policy can best be described in terms of the engineering model: politics maintained primacy, while research helped to legitimise the dominant policy approach. In other words, German immigrant integration research helped to engineer the German welfare state approach to integration. 
An institutionalised nexus between research and policy did not form until the persistent norm that Germany was and should not be an Einwanderungsland had been punctuated. However, this punctuation itself was not primarily an achievement of research-policy dialogues. It was largely an effect of political turnover at the end of the 1990s, when the CDU/ CSU government was replaced by the first ever SPD/Greens. Through the Süssmuth Commission, independent, academic expertise was able to play a role in this frameshift. Although the commission had an important effect on German discourse in terms of realising that the country had become an Einwanderungsland and that an integration policy was required, its direct impact on policy was limited. Firstly, the decision to establish this committee was contested - the political opposition saw it as an indication that government wanted to develop policy in an elitist, technocratic way, neglecting popular concerns about migration. Secondly, the committee adopted a highly rational, technocratic line of argumentation applicable to migration regulation and integration policies, which was, indeed, disconnected from multiplying public and political worries about social-cultural elements of integration and, particularly, preservation of German Leitkultur. This reflects the situation with the Dutch Blok Committee and underscores how hard it is to resolve intractable policy controversies through independent expertise - for the simple reason that this expertise tends to be contested as well.

The institutionalisation of a policy domain for migration and integration after the turn of the millennium also gave way to the institutionalisation of a research-policy nexus. The Süssmuth Commission, and its short-lived successor, the Immigration Council, raised awareness not only about the need for an integration policy, but also that policy-development required more systematic data provision and research. As such, the German Federal Office for Migration and Refugees established an in-house research group that would provide a first form of the institutional research-policy nexus in Germany. However, its main function would be to bolster the organisational legitimacy of this federal office within the emerging institutional policy domain. This reconfiguration of the German research-policy nexus is thus best described in terms of the bureaucratic model, involving a clear demarcation of the roles of research and policy and a clear political primacy. In this sense, the new nexus seems to reflect the broader German tradition of rational bureaucracy.

\subsection{The UK}

\subsubsection{The British race relations model}

The UK was one of the first European countries to recognise having become a multi-ethnic, multicultural society. This ethos was not so much 
cosmopolitan in nature but rather, closely tied to British legacy as having a culturally diverse empire in which 'race relations' had to be managed. Moreover, the UK has held on to the idea - and norm - of being a multicultural society much longer than most other European countries; only recently have there been indications that the UK is joining the assimilationist turn that is spreading across Western Europe (Joppke \& Morawska 2004).

\section{Universal British citizenship}

How have the British framed immigrant integration over the past decades? First, it is important to recognise that, like France, the UK has a long tradition of post-war immigration. In contrast to its history of emigration and colonisation around the world, the UK itself was confronted with significant immigration from its former colonies after World War II - an issue often phrased in the literature as 'the empire strikes back'. Like initial colonial migration to the Netherlands, most of these migrants were never seen as immigrants in the first place, perceived, instead, as subjects of the British Crown who had the right to settle in the UK. The 1948 British Nationality Act also provided equal legal, political and social rights to all citizens in the Kingdom as subjects of the crown (Geddes 2003: 32).

However, the levels of migration in the 1950s, the rise of public hostility towards 'coloured' migration (Freeman 1994) and the occurrence of a series of focus events in the late 1950s (Joppke 1999b) compelled the UK to implement more restrictive immigration policies. Compared to other European nations, the UK formulated some of the most restrictive policies, allowing only for a minimum of immigration - which was in sharp contrast to its earlier expansive immigration policies. Whereas in Germany the rationale for restrictive immigration policies was being an incomplete nation conflicted by the idea of becoming an immigration country, in Britain the rationale was based on a conception of the nation considered too wide to be accommodated by the state (ibid.: 62). As early as 1958, race riots in Nottingham and London's Notting Hill called attention to rising hostility towards colonial migration. This led to restrictive immigration policies between 1962 and 1981, eventually differentiating British citizenship as full citizenship, citizenship of British Dependent Territories and citizenship of British Overseas Territories, each category with distinct migration eligibilities.

An essential difference with other countries was that most immigrants in the UK already enjoyed formal citizenship. This meant that a different type of citizenship discourse emerged than in France and Germany, where citizenship was closely tied up with the issue of naturalisation. In the UK, the logic of immigrant integration was rather a form of legal, political and, ultimately, social rights, as in Marshall's (1964) 'Citizenship and social class', which predicated this progressive expansion of rights throughout various classes of British society. In contrast to the 'thick' citizenship 
conception in Germany and, to some extent, France, where citizenship was connected to issues of national identity, citizenship in the UK was relatively 'thin', or empty (Hansen 2003; Joppke 1999b; Small \& Solomos 2006). Consequently, framing the UK as a multicultural society went relatively uncontested, having deep roots in the legacy of multi-ethnic nationhood and indirect rule of the British Empire, which had left significant space for cultural pluralism (ibid.: 233).

\section{British 'multiculturalism-on-one-island': The race relations frame}

Adopting restrictive integration was an important condition for the UK's formulation of integration policies in the 1960s. As described by Brubaker (1992), the British model was externally exclusive and at the same time internally inclusive. Assimilation was rejected (in contrast to most other European countries, which at that time still conducted assimilationist policies), and a choice was made for a multiculturalist approach that was also distinctly national in nature, referring to the multicultural British society rather than to a connection between Britain and foreign cultures, such as in transnationalist or post-nationalist perspectives (Vertovec 2001); it was 'multiculturalism-on-one-island' (Favell 1998a). Until as late as 1999, British multicultural society was perceived as a 'community of communities' (Parekh 1999).

Colour and race were central to how the issue of immigrant integration was framed. Integration policy was framed primarily as an issue of improving race relations (Broeders 2001; Favell 1998a; Joppke 1999a). One of the reasons for this was that at the time of framing its first integration policies in the 1960s, the UK took a good look across the Atlantic. The contemporaneous US case was trying to cope with significant racial unrest (Bleich 2003). In the absence of citizenship as a defining and dividing characteristic, race and colour became the criteria for social classification. To some extent, this resembled the minoritarian logic of framing policy's social target groups in a way that would be developed in the Netherlands later on, with a focus on ethnic or cultural minorities. One important difference, however, is that minorities in the UK were not framed according to ethnicity, culture or religion but rather, on colour or race (Modood 2005). This was a racialisation of the immigrant issue, as opposed to the Netherlands' ethnic minorisation and the colour-blindness found in French Republicanism (Rath 1991).

The goal of British multiculturalism was to promote racial equality and equal opportunities and improve race relations (Soysal 1994: 54), rather than to contribute to the emancipation of racial groups, per se. 'Racial inexplicitness' proved to be a powerful norm (Kirp in Joppke 1999b: 226). This means that race was referred to as a general category and group particularism was avoided. One of the reasons for this was that British race relations policies were framed in the shadow of American racial unrest and 
that the UK wanted to avoid such racial consciousness in the interest of public order. This reflected the state's role in British society, which differed majorly from other European countries in that it was much more limited and residual, primarily taking care of issues such as public order. State interference in the private sphere, including, for instance, culture, was much more limited. A British judge once stated that 'England [...] is not a country where everything is forbidden except what is expressly permitted; it is a country where everything is permitted except where it is expressly forbidden' (Joppke 1999b: 233). One of the rationales for the British state to engage in immigrant integration in the first place was to preserve public order by managing race relations.

Instead of group emancipation, which was the aim of Dutch multiculturalist policies in the 1980s, the British sought 'welfare state integration' with much less government intervention (Joppke 1999b). Emphasis was on ensuring equal opportunities in spheres such as education, labour, housing and health care for all citizens, without carving out specific social groups within the British welfare state, an institution that is universalist and needsbased (Titmuss 1976). According to Soysal (1994: 55), the aim of the policy was 'that of providing opportunities for and removing obstacles to individual migrants' integration into existing institutions of society, so that they can gain those benefits already offered by the system'. In this vein, it specifically stressed the anti-discrimination issue. Three successive Race Relations Acts, in 1965, 1968 and 1976, established progressive legislation to limit discrimination, with the first two acts concentrating mainly on direct discrimination, but the third act also including indirect and statistical discrimination (Geddes 2003). The 1976 act also formulated positive action policies and established a Commission for Racial Equality (CRE) to monitor compliance to the Race Relation Act (ibid.: 46). Monitoring also invoked the question of social classification - so-called 'ethnic monitoring' as racial discrimination could only be monitored if there was some kind of registration of ethnic or racial minorities. Though contradicting the norm of racial inexplicitness, a question about race was included in the 1991 decennial population census in order to register racial belonging.

\section{The limits of British multiculturalism}

Developments in the last decade or so have drawn attention to the limits of British multiculturalism, contributing to a modest assimilationist turn in integration policies, like many other Western European countries (Joppke \& Morawska 2004). The imagined 'community of communities' has been further challenged by events such as the 1989 Rushdie Affair and a strengthened religious consciousness and activism on the part of British Muslims (Favell 1998b). As a religious category or community rather than a racial category, Muslims defy the British frame of race relations politics (Modood 2005). More and more, a process is unfolding to redefine the 
British imagined community by finding the minimum requirement of what 'being British' means and by searching for commonalities - more than compatibilities - in order to improve race relations (Joppke 2003; Singh 2004; Small \& Solomos 2006).

An advisory report to the government in 2001, entitled 'Community cohesion' (the so-called Cantle Report), sketched a grim picture of the British community of communities; it lacked a 'meta-community', where minorities and natives often lived 'parallel' lives in local British neighbourhoods, contributing to spatial segregation and polarisation between ethnic, racial and religious groups (see Joppke 2003). It rejected the current British laissez-faire approach to multiculturalism as a policy that would contribute to de facto apartheid (see Singh 2004: 56-57). In 2002, the national government issued a new memorandum on integration policy, 'Secure borders, safe haven', which adopted many elements of the Cantle Report and moved 'beyond multiculturalism'. It attempted to 'thicken' the British citizenship conception, referring to a national 'sense of belonging and identity' as a condition for British society to be able to receive and absorb immigration. Thus far, restricting immigration had been perceived as a condition for positive race relations; now the preservation of national identity was set as a condition for restricting immigration. In concrete terms, the government attributed a more central role to English language apprehension in integration and, in order to stress the 'thicker' citizenship conception, it also introduced more ceremonial naturalisation procedures (Hansen 2003).

\subsubsection{The construction of the British race relations frame}

\section{Early debate expansion and contraction}

On the international landscape, the British case stands out for the early point at which the debate about migration and immigrant integration expanded (politicisation), as well as how quickly its scale and scope again contracted (depoliticisation). Already by the late 1950s and 1960s, immigrant integration had become a major concern in the political field. This was largely in response to rising racial tensions among the British public (Freeman 1994), notably culminating in race riots in Nottingham and Notting Hill, orchestrated by anti-immigrant political forces. This led to polarisation between the Conservative Party and the Labour Party with the former pleading for tough restrictions on immigration and the latter warning against a rise in racism and discrimination (Geddes 2003).

A turning point came in 1968 when Enoch Powell, a right-wing Conservative, gave a speech in which he warned against 'rivers of blood'. If the native white population were to become even more marginalised in their cities and no proper action was taken, there could be bloody outcomes, as Powell warned (Favell 1998b: 105). According to Favell (ibid.), 
Powell linked migration and integration to the issue of public order. However, he also invoked more essentialist notions of 'the English people', in contrast to what he defined as inassimilable migrants from some former British colonies (Geddes 2003).

The stark politicisation invoked by Powell seems to have had a somewhat adverse effect. First, it was precisely concern with public order that incited British politicians to prevent inflammatory speeches such as those made by Powell, who was dismissed from his post and forced into an informal agreement to desist further politicisation of the issue (Favell 1998b: 106). Moreover, a fear was incited that 'playing the race card' could, in fact, undermine the current political establishment (Geddes 2003; Saggar 1992). Powell's politicisation contributed to a political agreement to remove this issue from the political agenda and out of the political field. Powell had ignored the rules of the political game forbidding any conflict about English nationhood that could potentially undermine the pluralist basis of the UK (Favell 1998b: 105) or that threatened the political capital of the current political establishment. In this sense, 'the Powell effect' resembled that of Le Pen in France, the Dutch anti-immigrant parties of the 1980s and the events at Solingen in Germany - all of which worked to strengthen the political anti-racism consensus rather than adopting tougher approaches to immigration and integration.

\section{The British race relations industry}

In response to the debate expansion of the 1960s, the British government embarked on an elite-crafted project of official multiculturalism (Joppke 1999a). Whereas Freeman (1994), argued that Britain's restrictionist immigration policies were a consequence of popular concerns about migration, its multiculturalist race relations politics rather seem to have been a consequence of elite anticipation (Favell 1998b; Statham \& Geddes 2006). It was a product of the consensus of political elites that originated in the 1960 s, rejecting both assimilation and a sharp anti-racist discourse. Like the republican model in France, British multiculturalist race relations were supported by a broad (left-right) political consensus that effectively excluded both the extreme left and the extreme right, and managed to sustain a stable policy monopoly for several decades to come. Indeed, in contrast to the issue's salience in the 1950s and 1960s, there would be very little political disagreement about these matters until after the turn of the millennium; the issue was, effectively, pacified.

As a consequence of the events in the 1950s and 1960s, an emergent structure in the political field devolved the issue to alternative venues (local government, scientific institutes), or to what would become known as 'the race relations industry'. According to Favell (1998b: 112), the British way of approaching this issue reflected 'a familiar utilitarian pattern in British political thinking, which emphasizes the consequential rather than 
the a priori deontological aspects of ethical and political reasoning'. Unlike France where such a priori deontological thinking has been common since the 1980s, partly due to the involvement of public intellectuals with a philosophical background, in the UK there was a preference for 'calculated, piecemeal, evolutionary, anti-philosophical pragmatism' (ibid.: 96). This meant, for instance, that symbolic deliberations on immigrant integration vis-à-vis national identity were systematically avoided; integration was instead framed as a Marshallian expansion of legal and political rights to social rights.

Joppke (1999a: 233-235) adds here the role that the British tradition of common law has played in this consequential logic. The UK's pragmatic legal tradition contrasted sharply with the prominence that constitutional legal principles have had in this domain in France and Germany. It allowed for much more flexibility and pragmatism in the legal accommodation of ethnic pluralism, without offering a venue for linking such issues to fundamental legal (and national) principles, such as embedding them in a formal constitution.

In the 1970 s and 1980 s, local venues were at the core of this logic of consequence in problem framing and in the depoliticisation of immigrant integration. Soysal (1994: 70) describes the way immigrant integration policy is organised in the UK as decentralised and society-centred, setting it apart from France and the Netherlands, in particular. There was no unitary policy structure for the coordination of British integration policy, except for CRE's legal, monitoring tasks at the national level. At the local level, welfare organisations or community relations councils (CRCs, later renamed RECs for Race Equality Councils) initially had a primary role in sustaining the welfare state integration of immigrants. This did not so much imply offering facilities but rather, aiding immigrants to make use of their rights.

Fear loomed that CRCs would exacerbate racial consciousness, again reflecting a similarity with the American case. As they were being replaced by immigrant self-organisations in the early 1980s (similar to what occurred in the Netherlands during the same period), their responsibility became ever more marginal. This resulted in local governments taking the primary role in promoting local race relations (Joppke 1999b: 238). Being reflected here was a British style of emphasis on welfare state integration in which local governments were key in administering large parts, such as education and housing (ibid.). Thus, devolving the integration issue to local venues supported the welfare state frame of integration and, subsequently, the logic of consequence in the political field.

It was also at this local level that ethnic elites were co-opted. This occurred by supporting the development of self-organisations, but also through the establishment of race relations units in local governments, for which representatives of immigrant racial minorities were selected (Soysal 
1994: 82). RECs are now also expected to represent minorities at the local level, though they do not have a formal role as a statutory body (ibid.: 74).

The race relations paradigm in British immigrant integration research

The field of immigrant integration research in the UK was first shaped by a sociology of race, which had its roots in sociology as well as anthropology (Small \& Solomos 2006). Like the Dutch anthropologists, the British sociology of race had its roots in the study of cultures abroad, namely in former British colonies. Only later would it turn towards the British home country (Bourne 1980). When the Institute for Race Relations (IRR) was established in 1952 (then still part of the Royal Institute for International Affairs), its sights were set on race relations abroad, whereas in subsequent decades it would become one of the leading institutes studying relations at home. Research in this early period, around the $1950 \mathrm{~s}$, concentrated on the cultural and racial 'otherness' of migrants (ibid.).

IRR would prove to be a cradle for British immigrant research, with pioneers such as Michael Banton, Richard Rose and Roy Jenkins. It was, however, torn apart in the early 1970 s by a boundary struggle provoking scientific as well as political questions. Its first report, 'Colour and citizenship' (Rose 1969), would set the tone for future integration research, defining colour as the guide to its analysis, as a legitimisation of the British race relations regime (Joppke 1999: 124). Instead of adopting a critical Marxist approach to issues such as institutional racism, the report sufficed with a rather factual policy-oriented recommendation, which was also explicitly stated (in a second version).

Any proposals for the amelioration of relationships between minorities and majority - and this book is intended principally as a constructive contribution towards policymaking in this field - must be justified in purely practical terms. [...] [and] have an application to the real problems of the adjustment process [...]. (see Bourne 1980: 337)

An impassioned internal struggle ensued between another IRR researcher, Jenkins and the research group centred around Rose. Gaining media attention, the conflict was about whether the crux of the study should be race relations, as in Rose's study, or racism, following Jenkins' ideas (ibid.: 339).

The so-called pluralist school, which focused on race relations, ethnicity and the transformation of the UK into a multicultural society rather than on institutional racism, appears to have won this boundary struggle. It managed to acquire funding from the Social Science Research Council (SSRC, then the main body for funding academic research in the social sciences) to establish a (then fully funded) Research Unit on Ethnic Relations, which would later become the Centre for Research in Ethnic 
Relations (CRER) (Bourne 1980; Ratcliffe 2001). At the same time, IRR would remain more on the margins of British integration research. Consistent with social science on the whole during the 1970s, leading researchers in this field were strongly inclined to take action. For instance, John Rex, director of CRER since the late 1970s and one of the UK's leading public intellectuals and sociologists in this period, fervently believed that the 'is' and 'ought' of sociological research could not be separated and sociology should maintain a societal commitment (Turner 2006). This may have contributed to the distinct orientation towards policy held by researchers in the field, as well as their being attuned to the political-ideological environment. For instance, Rex had dropped many of his critical Marxist ideas by the time he became CRER director (Favell 2001). CRER also maintained close ties with state agencies, which sometimes conflicted with the centre's academic sensibility, for instance, when disagreements transpired over use of the term 'Thatcherism' in a report from the 1980s (Ratcliffe 2001: 129). Critics argue that the involvement of researchers in the 'race relations industry' may have inadvertently contributed to racism and state control over race relations, while being distinctly out of touch with the black/racial target groups themselves (Bourne 1980). Just as in the Netherlands, the British government seems to have preferred expert expertise over ethnic expertise.

The dominance of race relations research appears not only to have legitimised the British race relations framing of integration policies, but also its guiding logic of consequence and pragmatism. Firstly, with a traditional emphasis on race and multiculturalism, race relations research provided a frame allowing the government to address immigrant integration without resorting to extreme-left anti-racism (with such Marxist theories already tamed in the boundary struggle that split the IRR) or policies of assimilation that would invoke the national identity taboo (which was never present in this research field). In addition, it supported policy development in an instrumental manner, with a sociological thesis that equality of opportunity and elimination of racial discrimination would support public order and prevent conflict ('rivers of blood') in a multicultural society (Favell 1998b: 116). It carried a positive claim that, with a sociologically informed intervention in race relations, public order could be secured (ibid.).

This contribution to the development of the British race relations frame is manifest in the long series of discrimination studies undertaken by CRER (also serving as funder), as well as the CRE and the Policy Science Institute (PSI), which was formerly Political and Economic Planning (PEP). PSI, in particular, has had an enormous direct and instrumental influence on policy developments, as two of its reports on discrimination and race relations $(1968,1977)$ were immediate precursors for two of the Race Equality Acts. Some of their later reports, however, appear to have been largely ignored (Ratcliffe 2001), perhaps due to the fact that PSI is 
known as a rather leftist think tank with little influence in periods of conservative rule.

An important issue in the relations between British social scientists and the British state has been the issue of ethnic monitoring, which is carried out by institutes such as CRE, in relation to policy purposes. On one hand, the racial inexplicitness of the British race relations frame means that race should remain a non-explicit category, not used for carving out specific groups. On the other hand, the monitoring of discrimination, and hence the evaluation of policy, would be impossible without some means of registering race as a social category. Social scientists and CRE, as a whole, had been lobbying government since the 1970s to include questions about race in the decennial population census (Ratcliffe 2001). It was not until 1991 that such a question was included. Still, it sparked significant conflict across the science-politics nexus. For instance, there were fervent objections to using the 1991 census data for a study on racial dynamics of labour and housing markets and related policy successes and failures, the latter of which would even have been censured by the Home Office (ibid.: 122-123). The dilemma between the need for ethnic monitoring and the norm of racial inexplicitness persists until this day. However, in the interest of anti-discrimination policies, the UK has been one of the first countries to introduce racial categories in national statistics (Joppke 1999b: 233).

The British case also reveals a persistent tendency towards what can be described as comitology. After nearly every focus event to occur in this domain, the government response has been to establish a committee, often led by a prominent politician but also calling on the expertise of leading researchers. For instance, following the 1981 riots in Brixton, the Scarman Committee was established. In 1985, the Gifford Committee was set up after further unrest. The Bradford Committee followed in 1995 and, in 2001, the Cantle Committee. Although several, especially those chaired by Scarman and Cantle, were quite influential, they often also seemed to be a substitute for political action (Ratcliffe 2001). Moreover, they rarely led to the type of the fundamental reflection seen in, for instance, the Nationality Commission and the High Council for Integration in France (Favell 1998b). As such, they slotted neatly into the British logic of depoliticisation and pragmatism.

There was, however, a clear rupture within British race relations research in the mid-1990s. This time, a new boundary struggle would split CRER. On one hand, a more critical strand of research emerged, so-called cultural studies on issues such as hybrid identities and identity politics among immigrants. The new school was led by researchers such as Paul Gilroy and Tariq Modood, and led to the establishment of the Centre for Contemporary Cultural Studies (Favell 2001; Small \& Solomos 2006). On the other hand, the cultural pluralists of race relations research (including Rex) also sought to expand their scope beyond the British territory, trying, 
for instance, to acquire more European research funding. In fact, CRER split precisely along these lines. To date, cultural studies has nevertheless acquired little resonance among policymakers, even despite its association with institutes such as PSI and IRR.

\section{The institutionalisation of a technocratic nexus}

In contrast to the other countries examined in this book, the level of politicisation in the UK has, since the 1960s, remained comparatively low. Direct involvement by social science researchers in policymaking, through various venues, has remained significant. Although the current assimilationist turn seems tied with progressive politicisation, until shortly after the turn of the millennium, the debate on immigrant integration (i.e. race relations) was conducted largely in technocratic terms. This involved attempts to manage race relations as a way to preserve public order, with several race relations acts based in important respects on reports from PSI. Recently, the government has shown renewed concern with 'evidencebased policymaking' in this domain (Boswell 2009). This became manifest in recent discussions on labour migration, which were highly influenced by reports from the Institute for Public Policy Research and the Research, Development and Statistics Directorate from the Home Office (ibid.: 109).

In the British research-policy nexus, boundaries between the two fields appear never to have been very sharp. First, personal networks and the academic credentials of specific experts often played a key role in researchpolicy dialogues. This reflects the broader British 'national style' in creating science-policy boundaries, whereby the credibility of science relies on 'the quality and reasonableness of the experts who provide the scientific evaluations' (Halffman 2005; Boswell 2009). These personal networks constituted the core of what Favell (1998b) describes as the elite-crafted project of British multiculturalism. Within these networks, the boundaries between research and policy were very diffuse, though academic credentials could also bring about a considerable amount of political capital. This clearly applies to a variety of leading social scientists in this field who have contributed significantly to the shaping of British migration policies (and immigrant integration research), including Rex and Bhikhu Parekh.

Secondly, the direct involvement of social science research in policymaking is also manifested in the organisation of an extensive in-house research capacity in the Home Office, particularly the Immigration Research and Statistics Service (IRSS) in the Research, Development and Statistics Department (Boswell 2009). In an in-depth analysis of how the IRSS functions and the knowledge utilisation of its reports, Boswell (ibid.: 124) shows how IRSS findings were often used to substantiate government positions, such as those in the field of labour migration. This contrasts with the German research group within the Federal Office for Migration and Refugees, which used findings mainly to legitimise its own organisational 
position within the emerging migration policy field. Within the largely technocratic setting of policy debate on labour migration, the IRSS research served as an important means for substantiating the liberal migration agenda of the New Labour government and convincing fellow organisations in the field of the need for a more liberal migration regime.

\subsubsection{The technocracy of British race relations}

The configuration of relations between British immigrant integration research and policymaking can best be described in terms of the technocratic model. Contrary to the other countries examined in this book, the debate on immigrant integration remained largely technocratic in the UK, at least up until recently. Integration was largely depoliticised and devolved to other venues like local governments and research bodies. In sharp contrast with the highly philosophical (deontological) logic of framing in France, the British followed a much more pragmatic (consequential) logic of framing. This involved a fervent belief that by managing race relations in a rational manner, public order could be maintained in the multicultural society and 'rivers of blood' could be averted. In this depoliticised setting, the British multicultural model of integration was the product of an 'elite project' to manage race relations. The expertise of researchers and specific research institutes played a key role in the development of these race relations policies. This included key roles for institutes like PSI, but also the CRER and, later, the IRSS in the Home Office. On some points in particular, researchers were able to exert significant influence on policy developments, such as in the case of the PSI reports on discrimination and the social-scientific lobbying for ethnic monitoring.

In this depoliticised setting, the boundaries between immigrant integration research and policy never became as articulated as they were in, for instance, Germany. Researchers were often involved in policymaking in very direct ways, often through highly personalised settings. This seems to reflect the broader British style of boundary regimes, where personalised academic credentials frequently have much more weight than formalised science-policy boundaries. This is manifested in the many instances where academic experts participated in government-oriented or government-associated commissions, such as the many examples raised by Bleich (2003) on how British academics and policymakers, alike, were influenced by the US race relations approach and, more recently, the example of the Commission on the Future of Multi-Ethnic Britain that was chaired by Parekh.

The race relations frame of immigrant integration was clearly an outcome of co-production between researchers and policymakers in the UK. After all, this frame was co-produced by what has been described as a 'race relations industry', in which researchers, but also local governments and organisations such as the CRE, played a central role. In the field of 
immigrant integration research, British sociologists of race have been at the core of co-producing a race relations frame. Having lost its most critical Marxist roots, the British sociology of race looked primarily to matters of culture, race and ethnicity in view of developing British multicultural society. Like Dutch social researchers of the same period, British sociologists had a strong sense of societal engagement as well as policy orientation. At an essential moment in the co-evolution of British immigrant integration politics and research, race relations specialists managed to acquire a dominant position. This occurred in the 1970s when IRR was struck by internal boundary struggles and CRER came on the scene, establishing a base for race relations research in the decades to come.

However, recent developments appear to have strained this technocratic symbiosis within the British race relations frame. On one hand, a gradual reframing of the British model occurred after the turn of the millennium, as renewed politicisation of the issue led to a re-ethnicisation of British policies, in search of commonalities and the minimum requirement of what 'being British' means. This politicisation and re-ethnicsation also seems to involve a punctuation of the technocratic and pragmatic style of policymaking. On the other hand, a new boundary struggle emerged in the British field of immigrant integration research: race relations researchers frequently looking towards Europe for funding versus cultural studies adopting a more critical perspective on British race relations politics. Just as in the Netherlands, the division of labour between social scientific research and politics in the UK appears to be characterised by a shift from symbiosis to a more antithetical relationship.

\subsection{Conclusions: Dutch exceptionalism?}

This chapter has explored the role of research in the construction - and reproduction - of specific frames of integration in France, Germany and the UK. The main objective was to find out whether the patterns of researchpolicy relations found in the Dutch case could also be identified in countries with very different national styles of boundary work. Could we identify more generalisable correspondences between the structure of researchpolicy relations and the culture of framing immigrant integration?

A first pattern found in several countries, notably the UK and the Netherlands, was the relationship between technocracy and multiculturalism. In both countries, a relation was found between, on one hand, a highly institutionalised issue domain with close ties between researchers and policymakers and, on the other hand, a multiculturalist type of problem framing that stressed specific characteristics of an ethno-cultural (Netherlands) or racial (UK) nature. Technocracy resulted in a depoliticised setting in both countries and a fervent belief in societal steering, be it the 
management of race relations in the UK or the state-led emancipation of minorities in the Netherlands. In addition, it involved a strong policy orientation and social engagement by researchers, exemplified, for instance, in PSI's and CRER's policy entrepreneurship in the UK and that of ACOM and WRR in the Netherlands. In both countries, multiculturalism was the product of 'elite technocratic management' (Favell 1998b), which, particularly for the Dutch case, made it vulnerable to criticism of being out of touch with popular concerns about the broader societal impact of immigration.

Apparently, there is something about technocracy wont to generate multiculturalist frames of immigrant integration. Regardless of the alleged national models of integration that dominate historical-institutionalist thinking, this analysis shows that it was the technocratic configuration of dialogues on immigrant integration that contributed to constructing a multiculturalist frame of integration. In fact, this technocratic configuration was produced in both countries only after short-lived periods of politicisation - following key trigger events such as the suburban riots in the UK and the Moluccan terrorist acts in the Netherlands. It was only then mostly 'behind closed doors' and by specific national elites - that the multiculturalist type of frames gained ground in both countries: namely, the race relations frame in the UK and the ethnic minorities frame in the Netherlands.

A second pattern found in several countries was that a lack of institutionalisation - deinstitutionalisation - of a migration policy domain tends to encourage linkages with broader societal issues such as the welfare state. This is what occurred in Germany until recently and in the Netherlands in the 1990s. In Germany, until about a decade ago, the Kein Eindwanderungsland myth prevented a policy domain from becoming institutionalised; in terms of both research and policy, this meant that issues of integration were mostly framed within the broader context of the welfare state. In the Netherlands, the integration policy of the 1990s also involved a more general (universalist) approach to integration, discontinuing or weakening the position of domain-specific actors such as the Minorities Policy Directorate and ACOM and, instead, delegating more responsibilities to generic policy departments and local governments. Lack of an institutional policy domain also implies that no institutional research-policy nexus can emerge. Indeed, in the Netherlands, the research-policy nexus of the 1980s was deconstructed in the early 1990s; in Germany, no nexus was constructed until shortly after the turn of the millennium. Research-policy dialogues in this setting mainly involved political primacy and a very selective use of data (e.g. from SCP in the Netherlands) or research that fitted into the dominant universalist problem frame (e.g. the dominant tradition of welfare state integration observed in German migration literature). 
Something about a lack of institutionalisation seems to prevent overemphasis on what is particular to immigrants and encourages what has been described in the Dutch case as a logic of equity in problem framing. This means that issues connected to broader societal themes tend to stress what migrants have in common with others as new citizens, rather than what differentiates them as ethnic or racial minorities. We can also speak of, in this respect, a co-evolution of immigrant integration research and policies in developing such univeralist type of frames. Clearly, although less direct than in technocratic types of research-policy relations, non-institutionalised research-policy dialogues contributed to the co-production of the welfare state integration frame in German migration policies and research and the integration paradigm in Dutch policies and research during the 1990s. It is doubtful, however, to what extent this co-evolution can be extended to all European countries. A key variable in the rise of universalism as found in Germany and the Netherlands of the 1990s is the structure of their welfare states. Both countries have extensive Bismarckian welfare states, involving extensive welfare facilities and a view of government as a 'compensator of first resort' (Leibfried 1992). This may explain why this type of universalist frame did not evolve in either the UK or France.

A third pattern was found between politicisation and the assimilationist turn that seems to be taking place in various Western European countries. Especially in the Netherlands but also in France, Germany and, to some extent, the UK, this shift was associated with a more antithetical relationship between research and policy. Politicisation often involved public questioning of scientific credibility and an aversion to inaccessible technocratic modes of policy development that would be out of touch with the public. This became evident in the Netherlands, where the Blok Committee sparked controversy about social researchers' reliability, alleging a multiculturalist bias and technocratic involvement in prior policy development. A similar controversy surrounded the Süssmuth Commission in Germany, spiralling criticism for its technocratic style that disregarded popular concerns about integration. France has, for even longer, been a scene of major boundary struggles involving open controversy between 'public intellectuals' in favour of an assimilationist frame and social scientists advocating a more pragmatic approach to integration; already for decades, boundary struggles have been manifest in issues of ethnic statistics in France.

Politicisation somehow created positive conditions for the rise of assimilationist frames in various countries. Especially in France and the Netherlands, the politicisation of immigrant integration spurred attention to the social-cultural facets of integration. This has generated a more philosophical or, as Favell (1998b) has described it, deontological logic of problem framing, concentrating on issues of great symbolic importance for the preservation of national identity (for instance, headscarves in France and dual nationality in the Netherlands). However, a deontological logic and 
the national focus of problem framing in political fields seems to be at odds with the broader development of an increasingly Europeanising field of integration research. This widening divide is evident in several countries, for example, in France's controversies about ethnic statistics and in the transnationalist and post-nationalist literatures that have evolved in particular in the Netherlands and Germany.

All cases revealed the institutionalisation of research-policy relations around specific frames. This applies to the welfare state frame of integration in German policy and research, the broad science-politics discourse coalition of the French Republican model, the British race relations industry and the Dutch technocratic symbiosis produced around the multicultural model of the 1980s. Clearly underscored is the argument made elsewhere (Favell 1999; Lavenex 2005) that immigrant integration research tends to be highly nationalised, co-producing the so-called 'national model of integration'. However, the ongoing Europeanisation of the domain of immigrant integration research does catalyse a denationalisation of research frames and a greater distance between research and politics in this respect.

This co-production of national frames has complicated critical frame reflection in the dialogues between immigrant integration research and policies. Research-policy dialogues rarely addressed the multiplicity of frames found in most countries. On one hand, researchers tended to ally themselves to one specific frame, often the one predominating their national setting. This was clearly manifest in the dominance of the race relations frame in British immigrant integration research, the welfare state frame in German research and the multicultural model in Dutch research of the 1980s. On the other hand, policymakers have had a tendency to pick and choose those research claims that support their policy discourse, rather than critically confronting their frames with potential alternatives. This was particularly evident in France, which had a blind spot for empirical social research that could contest the assumptions of the republican model. In various cases, though, the Europeanisation of immigrant integration research and the rise of transnationalist and post-nationalist frames accelerated the widening divide between research and policy in this respect.

The difficulties of engaging in frame reflective dialogues on immigrant integration have become obvious from the various committees established to 'reflect' on integration policies. The Blok Committee in the Netherlands, the Süssmuth Commission in Germany and the Stasi Commission in France were established to respond to the challenges being faced by national governments in taming the progressively politicised controversies on integration. Confronted with the multiplicity of frames involved in these controversies, each of these bodies chose to affiliate itself with a specific frame: universalist for the Süssmuth Commission and the Blok Committee, and a more assimilationist (republicanist) frame for the Stasi Commission. Yet, rather than encouraging frame reflection, these 
bodies contributed further to frame controversy. The fact that all fell into controversy clearly underscores the theoretical premise that intractable controversies cannot be resolved through re-examining 'the facts', as these facts tend to be selected and interpreted very differently by actors with different frames.

Can we conclude, then, that the Dutch case of research-policy dialogues on immigrant integration is exceptional? In most respects, it is not. The Netherlands reveals a type of national-level co-evolution between immigrant integration research and migration policies that was clearly present in other countries as well, despite this pattern of co-evolution following different national paths. We have also seen that research-policy dialogues in the Netherlands were neither more nor less 'reflective' than in other countries. Just as in surrounding countries, the role of research became contested in a situational setting characterised by frame conflicts. In particular, the tendency for research-policy relations to institutionalise around specific frames seems to have contributed to this.

What may have to some extent been exceptional is the ferocity of frameshifts and frame conflicts in the Netherlands. For in no other county were so many frameshifts found to occur in such a relatively short time. In fact, no single Dutch 'model of integration' could maintain itself in official policy discourse for more than a decade or so, whereas models in surrounding countries - though more dynamic than the historical-institutionalist literature suggests - were maintained for longer periods. Moreover, in no other country did frame conflicts become so intense (though France seems to approximate the Netherlands in this respect). This applies, in particular, to the fervent denunciation of multiculturalism, which was declared ex post a failure and blamed for many current Dutch integration problems. Related to the ferocity of frame conflicts, the role of research has become perhaps even more contested than in the other countries. 



\section{Conclusion: Towards reflective research-policy dialogues?}

This book has ventured into both the political and academic controversies on immigrant integration in the Netherlands over the past decades. Rather than honing in on a specific 'model of integration' or the famous - or infamous - Dutch multicultural model of integration itself, it has analysed the multiplicity of frames of immigrant integration. Immigrant integration is an essentially contested concept in the Netherlands, with policymakers adopting a new frame of integration almost once every decade or so. This significantly challenges the view in national and international literature that the Dutch case represents a coherent, consistent multicultural model. Modelling the Dutch case as such seems to be an ex post construction rather than an accurate depiction of formal policy discourse in the Netherlands. Indeed, although there was something like a multicultural model during most of the 1980s, it had already been abandoned by the beginning of the 1990s and was constantly contested by other frames of integration.

Furthermore, disaccord about how to frame immigrant integration was closely connected to controversies over mutual relations between research and policymaking in the field. In the Netherlands, immigrant integration research has been tied up with the framing of this issue as well as the construction of the Dutch multicultural model. Indeed, the development of both fields can, to some extent, be described as a pattern of co-evolution. However, Dutch immigrant integration research has become highly fragmented, with several frames of integration. This has complicated researchpolicy dialogues, particularly in the last decade. Researchers tend to affiliate themselves to specific frames, whereas policymakers tend to ignore research containing a frame other than their own. The recent controversies surrounding the Blok Committee, which defined Dutch policies as a relative success even though many political parties had denounced it as a failure, illustrates how easily research-policy dialogues can digress into a dialogue of the deaf.

Analysing such dialogues in the construction of specific frames, this book has tried to transcend the more historical-institutionalist modes of 'models thinking' that have acquired wide resonance in European immigrant integration research. From a structuralist-constructivist perspective, this study has taken such models as objects of analysis rather than as 
starting points for analysis, and has delved into the process of constructing the models as frames. This approach has proven highly valuable in understanding controversies surrounding immigrant integration and the multiplicity of frames involved. It has also shown how some cultures of framing immigrant integration tend to be associated with specific structures of relations between different types of actors.

\subsection{Beyond the Dutch multicultural model}

This book punctuates the image of a dominant, consistent and coherent Dutch multicultural model of integration. Instead, it has developed a view of the Netherlands' approach as contested and involving a multiplicity of frames. Contrary to the path dependency and policy coherency and consistency suggested by models thinking, this view emphasises dynamics, policy conflict and a high degree of inconsistency in the Dutch approach over the past decades.

In both policy and research there has been, besides the multiculturalist framing of integration, a long tradition of a more universalist framing of integration. More recently, assimilationist and transnationalist frames have evolved in both fields as well, contributing further to the multiplicity of frames making immigrant integration such an intractable controversy. Different frames have prevailed in distinct periods throughout the development of Dutch integration policies - multiculturalism in the $1980 \mathrm{~s}$, universalism in the 1990s and a more assimilationist frame since the turn of the millennium. In the field of research, this multiplicity was not so much manifested in periodic shifts but rather, in a progressive fragmentation of the research field, involving different research paradigms (minorities paradigm, integration paradigm, etc.) associated with specific experts, research institutes and advisory bodies.

The multicultural model, for which the Netherlands has become so internationally known, can be redefined as a discourse and situated in a particular historical and structural setting, rather than denounced altogether. Indeed, during the 1980s, a multiculturalist frame was dominant in both policy and research (though in both fields combined with important elements of universalist thinking). It was founded on the consensus of a relatively small network of researchers and policymakers who shared a concern for what was specific to the position of ethnic or cultural minorities the logic of minorities. Despite the fact that Dutch society had been depillarising rapidly since the late $1950 \mathrm{~s}$, this frame seemed to reflect how Dutch society had responded to social and religious pluralism earlier in the twentieth century. Vink (2007) explains this as a 'pillarisation reflex', meaning that this pillarisation frame was reinvented in response to immigration, so as to tame a potentially controversial policy problem. The idea 
of a pillarisation reflex in the framing of this issue cannot, of course, conceal that immigrant minorities in the Netherlands never really evolved into the well-organised and coherent pillars that had once characterised society. Furthermore, the dominant cultural-anthropological tradition in immigrant integration research contributed to the emphasis on cultural or ethnic minorities, without any connection to pillarisation.

In formal policy discourse, this multiculturalist model was abandoned in the early 1990s for a more universalist approach, stressing the 'good' or 'active' citizenship of individual migrants, especially in social-economic areas such as labour, education and housing. By 1989, WRR had denounced the decade's multiculturalist approach as largely ineffective, turning migrants into 'welfare dependents' and being too bogged down in 'symbolic debates'. This report, together with the national minorities dispute of the early 1990s, contributed significantly to the citizenship-oriented approach of integration policy seen in the same decade. In immigrant integration research more generally, there was a major emphasis on the participation of individual migrants as citizens in the social-economic sphere, as illustrated in the SCP reports. After the turn of the millennium and especially after the 'long year of 2002' and the Fortuyn revolution in Dutch politics, the multicultural model was denounced in even stronger terms. A 2003 memorandum stated that 'with the cultivation of the own cultural identities it is not possible to bridge differences [...] the unity of our society must be found in what the members have in common'. 'This statement clearly illustrates the assimilationist turn that Dutch policies experienced in the first years of the new millennium.

In Dutch immigrant integration research, the ongoing trend towards Europeanisation and internationalisation is contributing to a multiplicity of frames and a widening gap between the worlds of policy and research. Whereas politicisation is triggering a national framing of immigrant integration, as illustrated by the tight issue linkage between immigrant integration and national identity and social cohesion, immigrant integration research has witnessed the evolution of transnationalist and post-nationalist frames. These frames, at least to some extent, denationalise the process of immigrant integration, being at odds with the assimilationist turn in many integration policies in Europe. One of the mechanisms behind this trend is the co-optation of researchers in European research networks, sometimes funded by European organisations (e.g. European framework programmes for research funding that have financed as an example par excellence the IMISCOE Network of Excellence).

Thus, multiculturalism was only one of several frames to have influenced policy discourse and research in the Netherlands. As a policy discourse, the multicultural model has had significant influence on policy practices, especially in the 1980 s. There is some evidence that policies initiated in the $1980 \mathrm{~s}$ were effectuated in this period and were even 
continued until well after the multicultural policies of the minorities policy were formally abandoned. This is what Koopmans (2007) refers to as the strong tendency for 'path dependency' practiced in Dutch integration policies. For instance, immigrant language and culture instruction continued until after the turn of the millennium, albeit in different forms and with different wordings of its rationale. Whereas the goal was initially formulated as contributing to identity formation of migrants within the Dutch multicultural society, the rationale of the 1990s was reframed in terms of 'language transition' by first mastering the mother tongue in order to support the subsequent apprehension of Dutch as a second language.

However, the extent to which these practices continue to be inspired by a coherent multicultural model remains contested. To some extent, the national and local levels of integration policy seem to have followed different institutional logics. Whereas national policy discourse was inspired by politicisation, trigger events and a concern with grand themes such as national identity and culture, local policy discourse seems to have been much more pragmatic in nature, concerned with practical modes of problem-coping and a more instrumental policy logic. An important instance of divergence in this respect concerns the recognition of ethno-cultural groups and minorities organisations. In the early 1990s, national government formally adopted a more colour-blind citizenship approach, approaching migrants as citizens rather than as ethnic or cultural groups. This meant that various group-specific, tailor-made projects would have to be abolished. Yet, in practice, group-specific projects continued to proliferate (De Zwart \& Poppelaars 2007). Often, there was a pragmatic need for policy practitioners to concentrate on specific groups and cooperate with migrant organisations, to be able to 'reach' the policy targets and to acquire relevant knowledge and information about them (Poppelaars \& Scholten 2008).

Although these local practices often imply a de facto recognition of cultural groups, it would be a mistake to consider them real multicultural policies. Rather, they form more pragmatic attempts to conduct effective policies at the local level. They are not inspired by an ideology of multiculturalism or a legacy of pillarisation but rather, by the more pragmatic need to recognise groups and develop tailor-made projects to conduct effective policies and, as former Mayor of Amsterdam Job Cohen often put it very aptly, to 'keep things together'. They also show that the 1990s' citizenship approach did not institutionalise as a coherent policy model or become a true 'national model'. Neither did the multicultural paradigm of the 1980s.

The analysis of Dutch migration policies and research presented in this study shows the value of looking beyond 'national models of integration'. Bowen (2007) has argued that we should distinguish between models of integration policies and models for integration policymaking. We have now seen that these national models tend to distort our view as models of 
integration policies, as their emphasis on historical consistency and policy coherency tends to conceal the more dynamic and contested nature of policymaking. Instead, ideal-typical frames of immigrant integration, based on earlier typologies from Castles and Miller (2003) and Koopmans and Statham (2000), have been used here to empirically analyse how and why specific frames come and go in policy discourse and research. Thus, rather then attempting to capture the Dutch case in terms of a specific model, this book has emphasised the process of problem construction in research and policymaking. Ideal-typical frames are tools for analysis rather than standins for empirical analysis. Furthermore, this type of frame analysis also allows for the empirical study of models for policymaking. Indeed, we have seen that 'multiculturalism' was such a model or discourse for policymaking for some actors in the 1980s and, in fact, has remained so ever since. However, this does not legitimise the qualification of Dutch policy and research in terms of such a multicultural model.

\subsection{The Dutch research-policy nexus on the line}

The nexus between immigrant integration research and policymaking in the Netherlands has played a central role in what can be described as a 'co-evolution' of both fields. Dutch immigrant integration research has often been highly policy-oriented, able to ensure its policy relevance through diverse institutional channels, including expert committees and advisory bodies such as ACOM, WRR and SCP. At the same time, immigrant integration policymaking has been majorly influenced by immigrant integration research, notably in comparison to other policy fields. Much more than the ethnic expertise of migrant organisations, academic expertise was pivotal in several policy turning points. The initial urge for depoliticisation, the belief in societal steering of processes of integration and, of course, the ready availability of policy-relevant research seem to have been important causal factors in the role of this nexus.

The nexus between research and policy has, however, been shaped very differently over specific periods. Supported thus is the premise of scientific studies that, in empirical research, the role of science rarely accords to that long-held dogma of 'science speaking truth to power'. This book finds clear correlations between what Gusfield (1976) described as the structure of policymaking, including a specific role for academic expertise, and the culture of policymaking, or the framing of the immigrant integration issue. First, the technocratic structure of policymaking in the late 1970s and early 1980 s created a structural setting that allowed for a multiculturalist framing of integration. As already indicated by Guiraudon (1997) and supported here, this technocratic structure helped keep the policy debate behind closed doors. It limited not only the discussion's scale to a small network 
of policymakers and experts, but also its scope, concerned with what was specific to ethnic and cultural minorities and that which called for a logic of minorities. This structure helped sustain a policy equilibrium during the 1980 s, insulating the integration policy domain from the broader politics of welfare state retrenchment during that period.

Second, research again proved crucial in the universalist policy turn in the early 1990s, albeit with a very different configuration of the researchpolicy nexus. In this period, research fulfilled an enlightenment role that helped punctuate the structural equilibrium that had, until then, sustained multiculturalism. In particular, WRR furnished a venue for the agenda setting of a more universalist framing of immigrant integration. Thanks to its 'scientific' authority, WRR was considered a legitimate venue for raising a new frame that broke major taboos in this domain. The report attracted broad public and political discussion, but only years later were many of its main ideas adopted in government policy. Yet, WRR's enlightenment role created issue linkages between immigrant integration and the broader politics of welfare state retrenchment, prompting a very different logic of problem framing. Instead of stressing the particularities of migrants, the new universalist frame redefined migrants as citizens, focusing on their participation in general institutions rather than on group-specific measures.

Third, the recent assimilationist turn in Dutch integration policies also involved a reconfiguration of the research-policy nexus. Immigrant integration became the crux of a broader populist challenge to the Dutch political establishment. More than before, immigrant integration became politicised, with Dutch politicians and government eager to respond to the 'voice from the street', which populists claim had been ignored for too long. To inspire new political impetus to the integration policy, research was used very selectively to provide symbolic support to the assimilationist policy turn. Data on social-cultural integration, such as that from SCP, now became more meaningful. At the same time, a third WRR report developing a more transnationalist problem framing was ignored. Moreover, the role social researchers had played in policy development over the past decades became subject of political controversy. When a parliamentary investigative committee asked a research institute to evaluate the effectiveness of the integration policy, the credibility of immigrant integration research was put openly on the line; there were allegations of a multiculturalist bias and overly close involvement in the policy they would now have to evaluate. Interestingly, this boundary struggle afforded an important opportunity for constructing the Dutch multicultural model in hindsight. The model became a powerful counter-discourse against which new policy developments were juxtaposed. Moreover, it served to disqualify various actors that had previously been involved in this domain.

Analysis of the evolution of research-policy relations over the past decades indicates a growing disenchantment in the dialogue between 
immigrant integration research and migration policies. On one hand, there was the tendency of research-policy relations in this domain to create what others have described as 'paradigmatic closure' (Castles \& Wihtol de Wenden 2006). The Dutch case reveals a repeated institutionalisation of research-policy relations around specific problem framings. In different periods, specific research and policy actors often converged on the researchpolicy nexus surrounding a shared frame, while excluding other actors with rival frames. It created not only a policy monopoly that could remain relatively stable for some time (about a decade or so), but also had a structuring effect on the field of immigrant integration research itself. This was manifest, for instance, in the late 1970 s when policy developments contributed to the dominant position of the minorities paradigm. It was also exemplified during the early 1990s, with the virtual disappearance of rival paradigms (Rath 2001), when policy renewal also triggered boundary struggles in immigrant integration research to challenge the minorities paradigm and fortify the integration paradigm.

This paradigmatic closure provides an important obstacle to frame critical research-policy dialogues. When research-policy relations are institutionalised around a specific frame, research-policy dialogues tend to accentuate instrumental or secondary policy aspects, rather than the fundamental framing of the problem. In situations characterised by a multiplicity of frames, this can lead to dialogues of the deaf, where actors with different frames have disparate selections or interpretations of 'evidence'. This became particularly manifest in the controversies surrounding the parliamentary investigative committee established in 2004. Based on an independent study that developed a universalist perspective on the state of immigrant integration, this committee concluded that the integration process was advancing relatively successfully in the Netherlands. In particular, the study and the committee itself pointed to evidence of progress in the domains of education and labour participation. Yet, these findings were assessed very differently in the political arena, where a more assimilationist frame now prevailed, one that emphasised cultural and religious issues, matters largely ignored by the committee. Rather than catalysing a new impetus for policymaking, this committee provoked yet another episode in the ongoing controversies on immigrant integration. It sparked widespread arguments about policy failure or success. Here, the multiplicity of frames became manifest more than ever, though the research-policy dialogues never achieved the level of critical frame reflection.

On the other hand, this book has also shown a budding recognition that scientific knowledge on immigrant integration is uncertain and knowledge claims are becoming publicly contested. Traditional (positivist) models that adapt a fervent belief in societal steering and social science's contribution to rational problem resolutions are being traded in for more political primacy in mutual relations and cynicism about scientific expertise. The 
growing fragmentation in the field of immigrant integration research, along with the more open manifestation of knowledge conflicts, seems to have contributed to this trend. This reflects a broader inclination in Dutch politics straying from traditional models of societal steering and, in particular, away from technocracy and policymaking 'behind closed doors', something censured by populist politicians since the turn of the millennium.

This trend is aptly illustrated by a growing number of instances where the credibility of migration scholars and related institutes is openly put on the line. Not surprisingly, given the close co-evolution of immigrant integration research and policy in the Netherlands, the policy orientation of researchers and institutes was often at stake in such controversies or 'boundary struggles'. This certainly applies to the second WRR report from 1989, which was publicly denounced by ACOM as 'non-scientific', even though ACOM itself was blamed for being too entangled with the technocratic symbiosis of the minorities paradigm from the 1980s. Particularly severe was the boundary struggle surrounding the VWJ study on which the parliamentary investigative committee founded most of its conclusions. The credibility of the involved researchers was openly called into question because of their alleged multiculturalist bias and overly close involvement in the policy that they were now supposed to evaluate. Clearly, research became entangled in the ongoing frame conflicts. Immigrant integration research was now associated with a multiculturalist counter-discourse, one reinvented after the turn of the millennium to mark the assimilationist turn in Dutch politics.

\subsection{Dutch exceptionalism?}

An exploration on the relations between immigrant integration research and policies in France, Germany and the UK has indicated that the Dutch case of research-policy dialogues on migration and integration is not exceptional. Despite having very different national styles of research-policy relations, there seems to be a broader tendency in these countries to coproduce national models of integration, which may be relatively specific to the field of immigrant integration research. The UK has witnessed an institutionalisation of research-policy relations around its race relations frame that was remarkably similar to the Netherlands' technocratic symbiosis of the 1980s. Though less institutionalised, there was also a distinct coproduction of the welfare state integration frame in policy and research in Germany; similarly, French experts and intellectuals have formed strong support for the French Republicanist frame. This supports the claims made in other studies (Favell 2001; Lavenex 2005; Castles \& Wihtol de Wenden 2006), though the recent Europeanisation of immigrant integration research seems to be challenging this dominant national focus. 
The tendency to look for uniquely national models of integration and the challenge for such models to account for actual policy dynamics also does not seem exceptional. Even in France, where the republicanist model is perhaps even more resilient than the Dutch multicultural model, there seem to have been various models over the past decades, as well as a growing manifestation of knowledge conflicts among French migration scholars. Also for Germany and the UK, this book has shown that the national models of integration (differentialist and race relations frames, respectively) were the products of highly specific actor strategies and situational factors that aided the construction of these models, rather than these models being embedded in historical structures, per se. For instance, both France and Germany both had specific political factors that contributed to the construction of their models. In France, the republican model was reinvented in response to Le Pen's challenge to both the left and the right. And in Germany, the differentialist frame long persisted due to a political stalemate between the Christian Democrats and Socialists, who both wished to change this frame albeit in different directions. Recognising that national models of integration were the products of specific actor strategies and situational factors advances our understanding of the often dynamic and contested nature of migration policymaking. It also allows us to reconceptualise so-called national models of integration as 'discourses' that shape our understanding of immigrant integration problems, though are themselves also shaped by actors and factors advocating a specific problem frame. As the Dutch case has shown, such models can remain powerful as counter discourses invoked to help distinguish policy failures from policy successes.

The international comparison has also revealed more generalisable relationships between, on one hand, specific structures of policymaking and the role of research therein and, on the other hand, specific cultures of problem framing. In the Netherlands of the 1980s and in the UK up until quite recently, the technocratic configuration of the research-policy nexus permitted a multiculturalist problem frame; it kept policymaking behind closed doors and limited the scope to specific ethnic or racial traits of immigrants. Furthermore, in various instances, the rise of more universalist frames was associated with issue linkages between integration and broader topics such as welfare state politics. This was the case in the Netherlands in the 1990s, but to some extent also in Germany during the 1970s and 1980s. Finally, in all the countries examined (though perhaps to a lesser degree in Germany), a correlation was found between mounting politicisation and the assimilationist turn in immigrant integration policies. This occurred in France relatively early, while in the other countries politicisation was avoided up until quite recently, thus postponing the assimilationist turn until after 2000 . 
Though further research is required to better establish the relation between the structure and culture of migration policymaking, the relations found across the four countries examined in this book highlight the value of the structuralist-constructivist perspective applied in this book. Structuralconstructivism helps us reach beyond the historical-institutionalist thinking that has become so widespread in European immigrant integration research. It also helps us understand the much more dynamic ways in which our framing of immigrant integration is connected to the structural settings of our society. This seems particularly relevant for the domain of immigrant integration, as our understanding of the problem is so deeply entwined with policies - as this book has shown. Though undeniably associated with social-economic deprivation, discrimination, neighbourhood problems and various other 'real world' concerns, immigrant integration seems, first and foremost, a policy problem and, increasingly, also a political problem. It illustrates how contemporary governments struggle to develop policies to come to terms with this intractable, difficult-to-define social problem. As a policy issue, it has challenged traditional modes of governance that were aimed at rational societal steering and often involved technocratic processes of policymaking. Perhaps more than any other policy issue, immigrant integration has urged governments to respond to constant public and political swings and be more reflexive in terms of the multiplicity of frames it faces. For this reason, advancing our understanding of migration policymaking will not only be a challenge to migration scholars, but also to the fields of policy studies and political sciences in general.

A structural-constructivist perspective also allows us to perceive and interpret how our scientific understanding of immigrant integration is connected to broader societal structures. As already premised by Schneider and Ingram (1997) and many scholars of science studies, science tends to be far from 'exceptional'. Its findings tend to be shaped by rigorous scientific methods, as well as by the social conditions in which research takes place. This seems particularly valid for the field (rather than the discipline) of immigrant integration research, the development of which has been so closely connected to the development of migration policies. Social scientists, too, tend to frame problems like immigrant integration. As this book's analysis of national models of integration has shown, their frames are also likely to be influenced by the social settings in which actors frame problems. This is precisely why advancing our empirical and theoretical understanding of research-policy relations is of such great importance to the future development of immigrant integration as a research field. 


\subsection{Towards reflexive research-policy dialogues?}

A salient revelation of this study, in terms of research-policy relations and their effect on the framing of immigrant integration, is that these relations rarely achieve a level of critical frame reflection. This echoes the disenchantment that seems to have shrouded research-policy relations in the field of migration throughout Europe over the past decades. On one hand, researchers tend to be unaware of their own selective, normative problem frames and how they can be socially conditioned. We see this most obviously in a tendency towards 'paradigmatic closure' in national fields of immigrant integration research. On the other hand, politicians and policymakers are becoming selective in their use of scientific expertise, cherrypicking only those strands that fit their problem framing. Thus revealed is an important mechanism behind the production of national models of integration. More recently, the rapid politicisation of immigrant integration seems to have made policymakers even more reluctant to found their policies on scientific expertise. As the Dutch case has shown, the very use of scientific expertise in policymaking has become contested, seen as a mere relic of the technocratic modes of policymaking now vehemently rejected.

This book has therefore proposed to reconceptualise research-policy relations as dialogues. Primarily, this means that rather than research withdrawing into its ivory tower or policy reacting to the political swings of the day, research and policy have responsibilities to stay in dialogue. Given the uncertainty of social-scientific knowledge claims and the difficulties experienced by governments coming to terms with this intractable policy controversy, this book proposes an alternative to the dogma that science speaks truth to power. The perspective is one of 'making sense together' (Hoppe 1999), whereby research and policy synergistically reflect on various possible 'truths', rather than one claimed as the absolute truth. Secondly, to be in dialogue means that no structural factors constrain interaction to one specific frame or model. Rather, research-policy dialogues should be designed to cope with a multiplicity of frames and to take them as objects of reflection rather than to take one frame as a given - as a model of integration.

Critical reflection arises only when research-policy dialogues are constantly on the look out for the prospect of a 'lost frame' (Rein 2008). For situations characterised by a multiplicity of frames, research-policy dialogues should not advocate a specific frame but rather, various alternative frames to be articulated and critically confronted. In this way, researchpolicy dialogues can help policymakers and researchers to make sense together - of wicked policy controversies like immigrant integration. 



\section{Notes}

\section{Chapter 1}

I Data from 2005. Source: CBS StatLine.

2 Ibid.

3 Data from 200I. Source: Blok 2004: 249.

4 Data from 2005. Source: CBS StatLine.

5 Since 2003 , total emigration has exceeded total immigration and, since 2005, even the number of 'non-natives' leaving the Netherlands has surpassed the number of new immigrants (numbers include administrative corrections). Source: CBS StatLine.

6 Allochtoon (plural: allochtonen) is Dutch for 'allochthonous', derived from Greek allos, ('other'), and chthon ('earth', 'land') - to mean 'originating from another country'. In the Netherlands, the term 'allochtoon' is widely used to refer to non-Western immigrants and their descendants who reside in the country.

\section{Chapter 3}

I Foreign workers memorandum. Parliamentary document, TK II I969-I970, I0504, nr. I: I3.

2 ibid. Position of migrants from Surinam in the Netherlands and policy on the midto long-term. Parliamentary document, TK I976-1977, I4398. The problematic of the Moluccan minority in the Netherlands. Parliamentary document, TK I977-I978, I49I5, nr. 2.

3 Foreign workers memorandum. Parliamentary document, TK II I969-1970, I0504, nr. I: I2.

4 ibid.: Io.

5 Foreign workers memorandum; Memorandum of understanding. Parliamentary document, TK 1973-1974, 10504, nr. 9.

6 ibid.

7 Minorities memorandum. Parliamentary document, TK I982-1983, I6102, nr. 2I: Io.

8 Reply memorandum to WRR report 'Ethnic minorities' (I979). Parliamentary document, TK I980-1918I, I6102, nr. 6.

9 Minorities memorandum. Parliamentary document, TK I982-I983, I6102, nr. 2I: I07.

Io ibid.: Io

II ibid.: I2.

I2 ibid.: II. Caravan dwellers were added to this list for pragmatic reasons.

I3 ibid.: I2.

I4 ibid.: Io. This goes at least for how the minorities policy was framed on paper. It seems that, in practice, few specific policies were ever successfully implemented (Molleman 2003).

I5 For instance, it was believed that mother-tongue apprehension would support identity development among minorities and, as such, would contribute to the multicultural society.

I6 ibid.: I7. 
I7 ibid.: I2.

I8 ibid.: I07.

I9 'Action programme i988'. Parliamentary document, TK I987-I988, 20260, nr. 2.

20 In policy practice, there seems to have been a strong tendency to 'path dependence' (Snel \& Scholten 2005). This meant, among other things, that many programmes were simply continued and were only legitimised in different ways.

2I Government position on the WRR report 'Immigrant policy' (I989). Parliamentary document, TK I989-I990, 2I472, nr. 3.

22 ibid.

23 'Annual report minorities policy I994'. Parliamentary document, TK I993-I994, 23409, nr. 2.

24 The so-called 'Motion Apostolou'. Parliamentary document, TK I993-I994, 23409, nr. 9 .

25 Parliamentary document, TK I993-I994, 23409, nr. 9.

26 Parliamentary document, TK I994-I995, 2390I, nr. 3.

27 Parliamentary document, TK I998-I999, 26333 , nr. 2, nr. 4.

28 Contours memorandum. Parliamentary document, TK I994-I995, 2390I, nr. 3: 8.

29 ibid.: 24.

30 ibid.: 25 .

3I ibid.: 22-23.

32 ibid.: 4 .

33 ibid.: $2 \mathrm{I}$

34 Parliamentary document, TK 200I-2002, 28I98, nr. 2.

35 Parliamentary document, TK 2003-2004, 29203, nr. I.

36 Parliamentary document, TK 2003-2004, 28689, nr. I7.

37 Parliamentary document, TK 2003-2004, 29203, nr. I: 7.

38 ibid.: 8 .

39 ibid.: 8.

40 ibid.: 8-9.

$4 \mathrm{I}$ ibid.: Io.

42 ibid.: 9 .

43 ibid.: 7 .

44 ibid.: 8.

45 Interviews with Penninx and Köbben.

46 Interviews with ISEO director and a researcher of SCP.

\section{Chapter 4}

I Parliamentary document, TK I977-I978, I49I5, nr. 2.

2 Initially, ACOM was named the Advisory Committee on Cultural Minorities Research. The adjective 'cultural' was dropped several years later, as minorities were then mostly described as 'ethnic' rather than 'cultural'. ACOM (I979: 2) believed that the use of the term 'ethnic minorities' would be more 'exclusive and therefore more clear'.

3 This council was also called the Committee on Interdepartmental Division of Tasks and Coordination (also known as the Van Veen Committee).

4 Parliamentary treaties I2668, nr. 3: 4.

5 Prime Minister Den Uyl in the first chamber of Parliament, during debates on the law on the establishment of WRR (I976) (Adriaansens I997: 20-2I).

6 Parliamentary treaties I4IOO III, nr. 8. 
7 The recent appointment of a new WRR chairman led to questions in Parliament (Parliamentary document, TK 2003-2004, Question nr. I92I).

8 Parliamentary document, TK I969-1970, 10504, nr. I: 9.

9 ibid.

IO 'De problematiek van de Molukse minderheid'. Parliamentary document, TK I977I978, I49I5, nr. 2.

II Parliamentary document, TK I977-I978, I4915, nr. I3.

I2 Interview with Molleman.

I3 This article was published in Socialisme \& Democratie, a journal closely affiliated with the Social Democratic Party.

I4 Interestingly, nationality was not an argument in this definition of cultural minorities. Moluccan, Surinamese and Antillean migrants mostly had Dutch nationality. The cultural perspective on minorities was, at this time, important for the development of an all-encompassing approach towards cultural minorities: as soon as policy towards one cultural group had changed (Moluccans), others could not be left out. Interview with Entzinger.

I5 Parliamentary document, TK I980-I98I, I6I02, nr. 6: 5 .

I6 ibid.: 5-6.

I7 ibid.: 6.

I8 ibid.: 9 .

I9 ibid.: 5-6, I0.

20 Draft minorities memorandum I98I in Entzinger 1984: 122.

2I Parliamentary document, TK I982-I983, I6IO2, nr. 2I.

22 Interviews with chairman and secretary of ACOM.

23 Interview with researcher involved in ACOM during this period.

24 Interview with Penninx.

25 Interview with a member of ACOM.

26 Interview with ACOM chairman.

27 Interview with Penninx.

28 Interview with member of ACOM of migrant background.

29 Interview with Penninx; see also Tinnemans I994: 99.

30 Interview with Penninx.

3I Minutes of tenth meeting of WRR council, 23 May I978 (A-78/IO).

32 Internal memorandum of project group: 'Some notes about ethnic minorities in preparation of a memorandum to the council', I4 August 1978. See also minutes of second meeting of WRR council, I3 February I979 (A-79/3). See also contract between WRR and Penninx, 3 April I978; WRR archive (A-78/I0.3).

33 Interview with Penninx.

34 Internal memorandum to the advisory council (B-council) from the WRR chairman, I7 April I979 (B-79/I). See also minutes of sixth meeting of WRR council, 24 April I979 (A-79/6.2).

35 Minutes of tenth meeting of WRR council, 23 May I978 (A-78/10).

36 In fact, WRR made a strategic decision to present its report before ACOM's, which was originally scheduled to be presented first; interview with Entzinger.

37 Interviews with both staff members who took the initiative for this WRR study (one of whom was the project secretary) and with the project chairman.

38 ibid.

39 Minutes of the tenth meeting of WRR council, 23 May I978 (A-78/10). In this meeting, the director and the secretary of ACOM were present, as well as the author of the preparatory study for WRR.

40 This report was initiated by WRR itself, without a formal (or informal) advisory request from government; interview with project chairman. 
4I Minutes of the tenth meeting of WRR council, 23 May I978 (A-78/IO).

42 ibid.

43 Interview with Molleman.

44 Internal memorandum containing 'several notes on ethnic minorities in preparation of a memorandum to the council on a possible WRR project', I4 August I978.

45 Interview with involved staff member.

46 ibid.

47 Interview with WRR chairman who was also the project chairman.

48 ibid.

49 Minutes of third meeting of WRR council, I3 February I979 (A-79/3: 3-4).

50 ibid.

5 I ibid.

52 ibid.

53 Minutes of WRR staff meeting, I9 April I979: 2.

54 ibid.

55 Interview with WRR staff member.

56 Interview with Entzinger.

57 Interview with civil servant from CRM.

58 Interview with Penninx.

59 Various authors that are referred to in this section were involved with ACOM during this period. Therefore, any of their own publications are treated as primary sources for this research.

6o Interview with Molleman.

6I Interview with Entzinger (then secretary of ACOM).

62 Interview with WRR staff member.

63 Interview with Molleman.

64 ibid.

65 Reply memorandum to WRR report, Department of Home Affairs I980: 20.

66 Minorities memorandum I980, TK II, I982-I983, I6102, nr. 20: I66.

67 Letter from the Minister of Home Affairs, I2 March I980, Archives of the Department of Home Affairs.

68 Interview with a member of ACOM.

69 Interviews with director and a civil servant from the Minorities Policy Directorate.

70 Interview with secretary of the interdepartmental committee for the preparation of a reply memorandum to the WRR report.

7I Minutes of meetings of the interdepartmental committee for the preparation of a minorities policy, National Archives, General Affairs Department, Prime Ministers Cabinet, 7584-7592.

72 Interview with Penninx.

73 Interview with Molleman.

74 Interview with Penninx.

75 ibid.

76 'Discussion memorandum for the first meeting of the interdepartmental committee for the preparation of policy initiation of the Cabinet concerning the minorities policy', Department of Home Affairs, io August 1979 (Bi4790806r7).

77 Interview with Molleman.

78 Van (Kuik 1986: II8).

79 ibid.

80 'Molukse bijdrage aan de Minderhedennota genegeerd' ('Moluccan contribution to Minorities memorandum ignored'), NRC Handelsblad 6 September 1979. 'Minderheden zijn het spuugzat' ('Minority groups are sick and tired'), Elsevier 27 October I979. 'Molukkers diep beledigd door uitspraken van Wiegel' ('Moluccans 
deeply insulted by Wiegel's statements'), NRC Handelsblad 24 March I980. 'Te weinig aandacht voor minderheden' ('Too little attention on minorities'), NRC Handelsblad 20 December 1979. 'Beleid provoceert minderheden' ('Policy provokes minorities'), De Volkskrant I5 March I980.

8I Interview with Molleman.

82 ibid.

83 Parliamentary document, TK I980-198I, I6102, nr. 6: I9.

84 See also Penninx i988b: 47.

85 In Tinnemans 1994: 256.

86 Interview with WRR staff member. Earlier drafts of WRR report, Archives, WRR (A79/5.2).

87 Van der Zwan, in report of the temporary parliamentary research committee on the integration policy (2003), TK 2003-2004, 28689, nr. IO: I82.

88 Interview with the involved staff member.

89 ibid.

90 Minutes of seventh meeting of the WRR council, 8 May I979 (A-79/7: 2-4). See also interviews with the involved staff member.

9I Minutes of seventh meeting of the WRR council, 8 May I979 (A-79/7: 2-4).

92 Interview with the involved staff member.

93 ibid.

94 Parliamentary document, TK I980-I98I, I6IO2, nr. 6: 5-6, Io.

\section{Chapter 5}

Data from the Department of Social Affairs in WRR I989: I05.

2 Source: CBS Statline.

3 ibid.

4 Parliamentary document, TK I989-I990, 2I472, nr. 3.

5 'Nederland valt best mee' ('The Netherlands is not that bad'), De Tijd 9 March I990. 'Doelen minderhedenbeleid zijn achteraf te ambitieus' ('In hindsight minority policy targets are too ambitious'), De Volkskrant I3 May I989.

6 Interview with Molleman.

7 Interview with Penninx.

8 'Lubbers wil doorbraak van minderhedentaboe' ('Lubbers wants to end taboo on minorities'), Het Parool 26 March 1990.

9 'Groot debat over alle minderheden' ('Major debate on all minorities'), Haagsche Courant 7 October I99I.

Io Interview with Molleman.

II ibid.

I2 Parliamentary treaties, TK I986-I987, I9700VII, nr. 65.

I3 Contours memorandum: 28.

I4 Interview with Molleman.

I5 Parliamentary document, TK I989-I990, 2I472, nr. 3.

I6 ibid.: 9 .

I7 ibid.: 8.

I8 ibid.: 2.

I9 ibid.: I5.

20 Parliamentary document, TK I993-I994, 23684, nr. I, 2.

2I Interview with the director of the Minorities Policy Directorate.

22 Parliamentary document, TK I993-I994, 23684, nr. 2: 8.

23 ibid.: 25 . 
24 Interview with civil servant from the Minorities Policy Directorate. In contrast to the Minorities Policy Directorate, the Minister of Home Affairs appears to have supported the discontinuation of ACOM.

25 Interviews with two civil servants from CRM and Department of Home Affairs involved in research programming.

26 TK II, I987-I988, 20260, nr. 2.

27 Advisory request 'Ethnic minorities', I October I987 in WRR I989: 207.

28 WRR I989: 45. This has been described as structural integration (Fermin 1997: I89). Alongside structural integration, WRR (I989: 45) also distinguished cultural integration: 'mutual coordination of behaviour, values and preferences by the members of various ethnic groups'.

29 ibid.: Io.

30 ibid.

3 I ibid.

32 ibid.: 54 .

33 ibid.: 43 .

34 ibid.: 9 .

35 ibid.: 99

36 ibid.: 3I.

37 ibid.

38 ibid.: 9

39 ibid.

40 ibid.: 18.

4I ibid.: 6I.

42 Van der Zwan in TK 2003-2004, 28689, nr. Io: I80.

43 Parliamentary document, TK I99I-I992, 223I4, nr. 5, 9.

44 Van der Zwan in TK 2003-2004, 28689, nr. I0: I80.

45 Interview with Entzinger.

46 'Lubbers wil doorbraak van minderhedentaboe' ('Lubbers wants to end taboo on minorities'), Het Parool 26 March I990. 'Spoeddebat Kamer over minder softe uitspraak Lubbers' ('Emergency Chamber debate about Lubbers less-than-soft words'), De Volkskrant 27 March 1990.

47 ibid.

48 ibid.

49 'Lubbers: Verzuiling kan opnieuw succes zijn' ('Lubbers: Pillarisation can be successful again'), Trouw 28 October I99I.

50 Bolkestein gave his speech at the International Liberal Conference in Lucerne on 6 September I99I. A summary of the text of this speech appeared in 'Islamitische immigranten moeten integreren', ('Islamic immigrants must integrate'), NRC Handelsblad Io September I99I. A few days later, Bolkestein wrote a more in-depth article for another newspaper, 'Integratie van minderheden moet met lef worden aangepakt' ('Integration of minorities must be tackled with courage'), De Volkskrant I2 September I99I.

5 I ibid.

52 ibid.

53 ibid.

54 Derived from a report from the Haut Conseil a l'Intégration (Mariën I992: 4).

55 Interview with Entzinger.

56 ibid.

57 ibid.

58 Archives WRR. Minutes of thirteenth meeting of WRR council, I3 September I987 (A-87/13). 
59 ibid.

60 ibid.

GI ibid.

62 Minutes of staff meeting, 23 February 1989. Proposal accepted in fourth meeting of the WRR council, 28 February I989.

63 Interview with WRR staff member.

64 Minutes of sixteenth meeting of WRR council, 29 November 1988. Minutes of first meeting of WRR council meeting, I7 January I989. Minutes of fourth meeting of WRR council, 28 February I989.

65 Minutes of fourth meeting of WRR council, 28 February I989.

66 Interview with Han Entzinger.

67 Interview with staff member involved in this project group.

68 Interview with project secretary.

69 Interview with Han Entzinger.

70 Interview with Han Entzinger.

7I Minutes of fourth meeting of WRR council, 28 February I989 (A-89/4: 3).

72 Interview with project secretary.

73 ibid.

74 Interviews with project chairman, project secretary and staff member. See also Fermin (1997: 190).

75 Albeda (1989) 'Rechten en plichten van een nieuw integratiebeleid' ('Rights and duties of a new integration policy'), NRC Handelsblad i7 August I989.

76 Interview with chairman of the council who was also chairman of the project group.

77 Interview with project secretary.

78 ibid.

79 For some time, this WRR project carried the working title 'Ethnic minorities II'.

80 Archives WRR. (A-87/18.3a).

8 I Interview with secretary of ACOM.

82 Original title in Dutch is 'Kanttekeningen bij de wetenschappelijke waarde van het WRR rapport'.

83 'Rapport van WRR over minderheden nogal eenzijdig' ('WRR report on minorities rather one-sided'), De Volkskrant I3 June I989; 'WRR - nota over allochtonen bevat ideeën van borreltafel' ('WRR - memorandum on immigrants includes ideas from cocktail hour'), De Volkskrant 20 June 1989.

84 In a newspaper interview, Entzinger stated that 'soon, the Mediterranean Sea will be no wider than the Rio Grande', De Volkskrant I April i989.

85 As cited in 'Hoogleraar moet weg van vakgroep' ('Professor must leave department'), and 'Stammenstrijd over minderheden splijt vakgroep' ('Disagreements about minorities split department'), NRC Handelsblad 5 January I990: I,3.

86 Interview with chair of ACOM.

87 ibid.

88 ibid.

89 'Stammenstrijd over minderheden splijt vakgroep' ('Disagreements about minorities split department'), NRC Handelsblad 5 January I990: 3. See also 'Muffe sfeer hangt rond ruzie over hoogleraar' ('Stale atmosphere surrounds arguments concerning professor'), De Volkskrant II January 1990.

90 Interview with ACOM chair.

9I NRC Handelsblad 5 January I990.

92 This decision was taken by the dean of the involved faculty, who at that time was also a member of the WRR council.

93 Interview with ACOM secretary.

94 Interview with researcher of SCP. 
95 Interview with ISEO director.

96 Interview with SCP director.

97 ibid.

98 Van der Zwan in TK 2003-2004, 28689, nr. IO: I8I.

99 ibid.

Ioo ibid.

IOI Note that there were differences in terms of the extent to which Entzinger and Van der Zwan advocated a more obligatory approach, as well as which sanctions would be most suited to achieving it. Interview with Entzinger and Van der Zwan in TK 2003-2004, 28689, nr. IO: I8I.

I02 Van der Zwan in TK 2003-2004, 28689, hr. IO: I8I.

I03 Interview with Entzinger.

I04 ibid.

I05 Interview with civil servant from Minorities Policy Directorate.

Io6 Interview with Molleman. See also Molleman 1978.

I07 TK I986-1987, I9700, nr. 65.

I08 Interview with civil servant from Minorities Policy Directorate.

Io9 ibid.

IIO ibid.

III ibid.

II2 ibid.

II3 TK I990-I99I, 2I472, nr.I5.

II4 ibid.

II5 Interview with Minorities Policy Directorate director.

II6 ibid.

II7 ibid.

II8 ibid.

II9 'Integratie, een probleem van allereerste orde' ('Integration, a first-order problem'), Haagsche Courant I2 September I99I.

I20 ibid.

I2I Blok 2004: 75.

I22 Parliamentary treaties, TK I992-I993 (4-I67/I90) and (5-193/220).

I23 Among others: 'Doodgeknuffeld' ('Cuddled to death'), Elsevier i8 February I989; 'Etnische minderheden doodgeknuffeld' ('Ethnic groups cuddled to death'), De Volkskrant i8 June i988.

I24 NRC Handelsblad Io May I989.

I25 Interview with Entzinger. See also Van der Zwan in Blok, TK 2003-2004, 28689, nr. IO: I80.

I26 Mariën (I992: I0) names the 'logic of equity' in an analysis of this debate.

\section{Chapter 6}

I Source: CBS Statline, data 2004 .

2 ibid.

3 Elsevier 25 August 200I; De Volkskrant 2 November 200I; De Volkskrant 9 February 2002.

4 Parliamentary document, TK 2002-2003, 28689, nr. I.

5 Interview with researcher from SCP.

6 'De multiculturele illusie' ('The multicultural illusion'), De Volkskrant I7 February 2000; 'De multiculturele illusie: Een pleidooi voor aanpassing en assimilatie' ('The 
multicultural illusion: A plea for adaptation and assimilation'), Essay. Utrecht: FORUM, 2000: 5-26.

7 Interview with SCP director.

8 Schnabel (2000) makes this distinction in terms of A-culture (complete assimilation), B-culture (some assimilation, integration) and C-culture (segregation).

9 This was a project together with IMES and the European Research Centre on Migration and Ethnic Relations (ERCOMER).

Io Among others, 'Arbeidsmarktpositie van Turken in Nederland en Duitsland' ('Labour-market position of Turks in the Netherlands and Germany') (SCP 2007); 'De sociale atlas van vrouwen uit etnische minderheidsgroepen' ('The social atlas of women from ethnic minorities') (SCP 2006); 'Grens overschrijdende huwelijken' ('Cross-border marriage') (SCP 2003); 'Heb Uw naaste lief?' ('Love thy neighbour?') (SCP 2005).

II WRR works in close relation to the government. For instance, it presents reports to the government, which are issued directly to the Council of Ministers. However, in formal terms, its reports can also be addressed to Parliament.

I2 De Volkskrant 30 March 2000.

I3 Parliamentary document, TK 2002-2003, 28600, nr. 24.

I4 Parliamentary treaties, TK I9 September 2002, 3-I82.

I5 Parliamentary document, 200I-2002, TK 28689, nr. I

I6 Parliamentary document, TK 2003-2004, 28689, nr. II: 5.

I7 Paul Scheffer has a background in both academics and politics (being a member of the Social Democratic Party).

I8 'Het multiculturele drama' ('The multicultural drama'), NRC Handelsblad 29 January 2000.

I9 ibid.

20 Parliamentary document, TK I999-2000, 27083, nrs. I, 2.

2I Party political programme of LPF 2002: 5 .

22 'Werken aan vertrouwen: Een programma van aanpakken', ('Building trust: A programme of getting things done?'), Agreement of the government coalition of LPF, CDA and VVD 2002: I3.

23 ibid.: I3. The government remained reluctant to use 'assimilation', possibly because of its negative connotations. In fact, it even repeatedly denounced the concept as such. This is contrary to SCP, which had earlier embraced the concept of assimilation. Nonetheless, based on the operationalisation of assimilation as one possible framing of immigrant integration, it can be reasonably claimed that government did in fact adopt an assimilationist problem frame.

24 Parliamentary document, TK 2003-2004, 29203, nr. I: 7.

25 ibid.: 8

26 Parliamentary document, TK 2003-2004, 28689, nr. I7: 6.

27 ibid.: 3.

28 ibid.: 5-6.

29 Interview with project chairman.

30 ibid.

3 I ibid.

32 Interview with staff member.

33 Minutes of meeting of advisory council of WRR (B-98/2).

34 Memorandum from the project group chairman to WRR council, 7 March 2000 (A००/4.2).

35 Memorandum from WRR chairman to WRR council, I6 March I999 (A-99/6.4).

36 ibid.

37 Minutes of sixth meeting of WRR council, 23 March I999 (A-99/6). 
38 ibid.

39 ibid.

40 Memorandum to WRR council, I8 August I999 (A99/13.3).

4I Memorandum to WRR council on the progress of the project group, 20 April I999 (A99/8.2).

42 Minutes of sixth meeting of the council, 1999 (A-99/6).

43 Interview with project chairman.

44 Interviews with project secretary and several staff members.

45 Interview with project chairman

46 Minutes of seventeenth and nineteenth meeting of WRR council, I998 (A-98/I7) and (A-98/19). See also interview with project chairman.

47 Interview with staff members.

48 Minutes of thirteenth meeting of WRR council, 3 July $200 \mathrm{I}$ (A-OI/I3).

49 ibid.

50 Interviews with project chairman and staff members. See also minutes of meeting of the project group, I6 May 2000.

5I Interview with WRR director, who was also the project chairman.

52 Interview with staff member.

53 Interview with researcher from SCP.

54 Interview with SCP director.

55 ibid.

56 ibid.

57 Parliamentary document, TK 2003-2004, 28689, nr. 8-9: I6.

58 Interview with Entzinger.

59 Interview with researcher from the VWJ.

6o ibid.

6I Parliamentary document, TK 2002-2003, 28600, nr. 24.

62 Parliamentary document, TK 2003-2004, 28689 nr. 8-9, p. 9.

63 ibid.: nr. I.

64 Parliamentary document, TK 2002-2003, 28689, nr. 2.

65 The committee was established on I8 December 2002 and had to report on its first tasks as early as 23 January 2003.

66 Parliamentary document, TK 2003-2004, 28689, nr. 8-9: I2.

67 ibid.: II.

68 Interview Blok Committee chairman.

69 ibid.

70 ibid.

7 I ibid.

72 Parliamentary document, TK 2003-2004, 28899, nr. 8-9: I6.

73 ibid.: $19-20$.

74 ibid.

75 ibid.: 2I-22.

76 ibid.

77 Interview with involved parliamentarian, 'Lazrak houdt het voor gezien', Vrij Nederland 20 September 2003.

78 'Harde kritiek uit Kamer op "naïef" rapport' (Harsh criticism from Chamber of "naive" report'), NRC Handelsblad i9 January 2004: 6.

79 'Makelaars in minderheden' ('Minority brokers'), De Volkskrant i9 September 2003.

80 Parliamentary hearings, 6 April 2004, 63-4IO2.

8I Parliamentary hearings, 6 April 2004, 63-4094.

82 Parliamentary hearings, 6 April 2004, 63-4127. 
83 Interview with chairman of WRR project group and civil servant from the Minorities Policy Directorate.

84 Interview with chairman of WRR project group.

85 The ISEO was left out and eventually dissolved.

86 Parliamentary treaties, 3I August 2004, 92-5932.

87 Parliamentary treaties, 6 April 2004, 63-4II2.

88 Migrantenstudies 2002, special issue I8: 2.

\section{Chapter 7}

I Original title in German is 'Integration, begrenzung des zuzugs, hilfe bei freiwillige rücckeh'.

\section{Chapter 8}

I TK 2003-2004, 29203: 8[0]. 



\section{References}

Abelson, D. E. (2002), Do think tanks matter? Assessing the impact of public policy institutes. London: McGill-Queen's University Press.

Adriaansens, H. (1997), 'Distantie en nabijheid', in H. Adriaansens (ed.), Mosterd bij de maaltijd, 59-78. The Hague: SDU.

Advies Commissie Onderzoek Minderheden (1989), Een beter beleid? The Hague: Staatsuitgeverij.

Advies Commissie Onderzoek Minderheden (1979), Advies onderzoek minderheden. The Hague: Staatsuitgeverij.

Amiraux, V. \& P. Simon (2006) 'There are no minorities here: Cultures of scholarship and public debate on immigrants and integration in France', International Journal of Comparative Sociology 37 (3-4): 191-215.

Andeweg, R. B. \& G. A. Irwin (2005), Governance and politics of the Netherlands. Houndmills: Palgrave Macmillan.

Association of Dutch Municipalities (VNG) (2003), De lokale agenda integratiebeleid. The Hague: ADM.

Baumgartner, F. R. \& B. D. Jones (2002), Policy dynamics. Chicago: University of Chicago Press.

Baumgartner, F. R. \& B. D. Jones (1993), Agendas and instability in American politics. London: University of Chicago Press.

Baumgartner, F. R., Green-Pedersen, C. \& B. D. Jones (2006), 'Comparative studies of policy agendas', Journal of European Public Policy 13 (7).

Beck, U. (1992), Risk society: Towards a new modernity. London: Sage.

Becker, F., W. Van Hennekeler, M. Sie Dhian Ho \& B. Tromp (2002), Transnationaal Nederland: Immigratie en integratie. Amsterdam: De Arbeiderspers.

Berger, P. \& T. Luckmann (1966), The social construction of reality: A treatise in the sociology of knowledge. London: Allan Lane.

Bertossi, C. (2009), 'La république "modèle" et ses discours modélisants: L'intégration performative à la française', Migrations Société 21 (122).

Bertossi, C. \& J. W. Duyvendak (2009), 'Modèles d'intégration et intégration des modèles? Une étude comparative entre la France et les Pays-Bas', Migrations Société 21 (122).

Beune, H. H. M. \& A. J. J. Hessels (1983), Minderheid: Minder recht? The Hague: SDU.

Birkland, T. A. (1998), 'Focusing events, mobilization and agenda setting', Journal for Public Policy 18 (1): 53-74.

Bleich, E. (2003), Race politics in Britain and France: Ideas and policy-making since the 1960s. Cambridge: Cambridge University Press.

Bleich, E. (2002), 'Integrating ideas into policy-making analysis: Frames and race policies in Britain and France', Comparative Political Studies 35 (9): 1054-1076.

Blok, S. (2004a), Bruggen bouwen: Eindrapport van de tijdelijke parlementaire onderzoekscommissie integratiebeleid. The Hague: SDU.

Blok, S. (2004b), Bruggen bouwen: Verslag gesprekken en hoorzittingen. The Hague: SDU.

Blume, S. S., R. P. Hagendijk, \& A. A. M. Prins (1991), 'Political culture and the policy orientation in Dutch social science', in P. Wagner (ed.), Social sciences and modern states: 
National experiences and theoretical crossroads, 168-190. Cambridge: Cambridge University Press.

Böcker, A. \& D. Thränhardt (2003), 'Is het Duitse integratiebeleid succesvoller, en zo ja, waarom?: Reactie op Koopmans', Migrantenstudies 19 (1): 33-44.

Bommes, M. \& E. Morawska (2005), 'Introduction', in M. Bommes \& E. Morawska (eds.), International migration research: Constructions, omissions and the promises of interdisciplinarity, 1-16. Aldershot: Ashgate.

Bootsma, P. (2000), De Molukse acties: Treinkapingen en gijzelingen 1970-1978. Amsterdam: Boom.

Boswell, C. (2009), The political uses of expert knowledge: Immigration policy and social research. Cambridge: Cambridge University Press.

Bourdieu, P. (2004), Science of science and reflexivity. Chicago: University of Chicago Press.

Bourdieu, P. (1988), Homo academicus. Cambridge: Polity Press.

Bourdieu, P. (1977), Outline of a theory of practice. New York: Cambridge University Press.

Bourdieu, P. (1975), 'The specificity of the scientific field and the social conditions of the progress of reason', Social Science Information 14 (6): 19-47.

Bourdieu, P. \& L. J. D. Wacquant (1992), An invitation to reflexive sociology. London: University of Chicago Press.

Bovenkerk, F. (1987), Een eerlijke kans? The Hague: ACOM.

Bovenkerk, F. (1984), 'Rassen of klassen?', Intermediair 20 (47): 31.

Bovenkerk, F. (1978), Omdat zij anders zijn: Patronen van rasdiscriminatie in Nederland. Meppel: Boom.

Bovenkerk, F. (1974), Gastarbeid, terugkeer en ontwikkelingssamenwerking: Een kritische inventarisatie van gangbare opvattingen en beleidsvoorstellen op korte termijn. The Hague: Employment Projects for Regional Development.

Bovenkerk, F. \& L. Brunt (1983), 'Where sociology falls short: How Dutch sociologists observe social reality', Netherlands Journal of Sociology: Sociologia Neerlandica 19: 6578.

Bovenkerk, F., F. Buijs \& H. Tromp (1990), Wetenschap en partijdigheid. Assen: Van Gorcum.

Bovenkerk, F., R. Miles \& G. Verbunt (1991), 'Comparative studies of migration and exclusion on the grounds of 'race' and ethnic background in Western Europe: A critical appraisal', International Migration Review 25 (2): 375-391.

Bourne, J. (1980), 'Cheerleaders and ombudsmen: The sociology of race relations in Britain', Race \& Class 21 (4): 431-452.

Bowen, J. (2007), 'A view from France on the internal complexity of national models', Journal of Ethnic and Migration Studies 33 (6): 1003-1016.

Bowen, J. (2006), Why the French don't like headscarves: Islam, the state and public space. Princeton: Princeton University Press.

Brubaker, R. (1992), Citizenship and nationhood in France and Germany. Cambridge: Harvard University Press.

Castles, S. \& M. J. Miller (2003), The age of migration: International population movements in the modern world, 3rd ed. New York: Palgrave Macmillan.

Castles, S., \& M. J. Miller (1993), Age of migration: International population movements in the modern world. London: Macmillan.

Castles, S. \& C. Wihtol de Wenden (2006), 'Framing international migration: From national models to transnational critique', in E. Vasta \& V. Vuddamalay (eds.), International migration and the social sciences, 222-251. Hampshire: Macmillan.

Choenni, C. E. S. (2000), 'Ontwikkeling van het rijksoverheidsbeleid voor etnische minderheden 1975-2000', in G. A. V. N. Beets (ed.), Bevolkingsvraagstukken in Nederland anno 2000:Werkverband periodieke bevolkingsvraagstukken, 131-144. The Hague: 58 WPRB, NIDI. 
Choenni, C. E. S. (1987), 'Een allochtone visie over onderzoek en onderzoeksbeleid inzake allochtonen', in P. Schnabel (ed.), Gezondheids(zorg)-onderzoek etnische minderheden: Verslag invitational conference 'Gezondheids(zorg)onderzoek etnische minderheden', 2643. Utrecht: NcGv.

Cobb, R. W. \& C. D. Elder (1983), Participation in American politics: The dynamics of agenda-building. Baltimore: The Johns Hopkins University Press.

Collins, H. M. (1983), 'The sociology of scientific knowledge: Studies of contemporary science', Annual Review of Sociology 9: 265-285.

Commission de la Nationalité (1988), 'Être français aujourd'hui et demain: Rapport de la Commission de la Nationalité', Paris: Union générale d'editions.

Council for Public Government (ROB) (2001), Ethnicity, bonds and citizenship. The Hague: ROB.

Council for Social Development (RMO) (2005), Unity, diversity and ties. The Hague: RMO.

Couwenberg, S. W. (1982), 'Het vraagstuk der etnische minderheden', Civis Mundi 21 (5): 169-212.

Dagevos, J. (2001), Perspectief op integratie: Over de sociaal-culturele en structurele integratie van etnische minderheden in Nederland (vol. W 121). The Hague: WRR.

De Beer, J. (1998), 'Migratie naar Nederland', in R. Penninx (ed.), Etnische minderheden en de multiculturele samenleving, 237-258. Groningen: Wolters-Noordhoff.

De Beus, J. (1998), De cultus van vermijding: Visies op migrantenpolitiek in Nederland. Utrecht: FORUM.

De Hart, B. (2004), 'Political debates on dual nationality in the Netherlands (1990-2003)', in A. Böcker, B. De Hart \& I. Michalowski (eds.), 'Migration and the regulation of social integration', IMIS-Beiträge 24, special issue: 149-162.

De Hart, B. \& B. Prins (2005), 'Onderzoeker tussen wetenschap en beleid: Rondetafelgesprek in de Waag op de Nieuwmarkt te Amsterdam, 12 mei 2005', Migrantenstudies 21 (4): 178-194.

De Jong, W. (2002), 'Drie WRR-rapporten vergeleken', Migrantenstudies 18 (2): 78-86.

De Zwart, F. (2005), 'The dilemma of recognition: Administrative categories and cultural diversity', Theory and Society 34 (2): 137-169.

Den Hoed, P. (1995), Bestuur en beleid van binnenuit: Een analyse van instituties. Meppel: Boom.

Donselaar, J. \& R. P. Wolff (1996), Reacties op racistisch geweld: Het perspectief van allochtonen. Amsterdam: Het Spinhuis.

Dunn, W. N. (1994), Public policy analysis: An introduction. Englewood Cliffs: Prentice Hall.

Duyvendak, J. W. \& P. W. A. Scholten (2009.) 'Le modèle multiculturel d'intégration néerlandais en question', Migrations Société 21 (122).

Duyvendak, J. W. \& L. Veldboer (2004), Meeting point Nederland: Over samenlevingsopbouw, multiculturaliteit en sociale cohesie. Amsterdam: Boom.

Edelman, E. (1988), Constructing the political spectacle. Chicago: University of Chicago Press.

Edelman, E. (1977), Political language: Words that succeed and policies that fail. New York: Academic Press.

Engbersen, G. \& R. Gabriëls (1995a), 'Voorbij segregatie en assimilatie', in G. Engbersen \& R. Gabriëls (eds.), Sferen van integratie: Naar een gedifferentieerd allochtonenbeleid, 1548. Meppel: Boom.

Engbersen, G. \& R. Gabriëls (eds.) (1995b), Sferen van integratie: Naar een gedifferentieerd allochtonenbeleid. Meppel: Boom.

Entzinger, H. (2006), 'The parallel decline of multiculturalism and the welfare state in the Netherlands', in K. Banting \& W. Kymlicka (eds.), Is multiculturalism bad for the welfare state? Oxford: Oxford University Press. 
Entzinger, H. (2005), 'Changing the rules while the game is on: From multiculturalism to assimilation in the Netherlands', in M. Bodemann \& G. Yurkadul (eds.), Migration, citizenship, ethnos: Incorporation regimes in Germany, Western Europe and North America. New York: Palgrave MacMillan.

Entzinger, H. (2003), 'The rise and fall of multiculturalism: The case of the Netherlands', in C. Joppke \& E. Morawska (eds.), Toward assimilation and citizenship: Immigrants in liberal nation-states. Hampshire: Palgrave.

Entzinger, H. (2002), Voorbij de multiculturele samenleving. Assen: Van Gorcum.

Entzinger, H. (1984), Het minderhedenbeleid: Dilemma's voor de overheid in Nederland en zes andere immigratielanden in Europa. Meppel: Boom.

Entzinger, H. (1981), 'De ACOM als voorbeeld van onderzoeksprogrammering; maar wat levert het nu op aan kwaliteitsverhoging?', in B. W. Frijling \& C. Rottländer-Meyer (eds.), Sociaal beleidsonderzoek:Luxe of noodzaak? Zoetermeer: Actaboek.

Entzinger, H. (1975), 'Nederland immigratieland? Enkele overwegingen bij het overheidsbeleid inzake allochtone minderheden', Beleid \& Maatschappij 2 (12): 326-336.

Entzinger, H. \& J. Van der Meer (2004), Grenzeloze solidariteit: Naar een migratiebestendige verzorgingsstaat. Amsterdam: De Balie.

Essed, P. \& K. Nimako (2006), 'Designs and (co)incidents: Cultures of scholarship and public policy on immigrants/minorities in the Netherlands', International Journal of Comparative Sociology 47 (3-4): 281-312.

Ezrahi, Y. (1990), The descent of Icarus: Science and the transformation of contemporary democracy. Cambridge, Mass.: Harvard University Press.

Faist, T. (2000), The volume and dynamics of international migration and transnational social spaces. Oxford: Clarendon Press.

Faist, T. (1994), 'How to define a foreigner? The symbolic politics of immigration in German partisan discourse, 1978-1992', West European Politics 17 (2): 50-72.

Fassin, D. (1999). 'Good to think: The American reference in French discourses of immigration and ethnicity', in: C. Joppke \& S. Lukes (eds.), Multicultural questions, 224-241. Oxford: Oxford University Press.

Favell, A. (2005), 'Integration nations: The nation-state and research on immigrants in Western Europe', in M. Bommes \& E. Morawska (eds.), International migration research: Constructions, omissions and the promises of interdisciplinarity. Aldershot: Ashgate.

Favell, A. (2001), 'Integration policy and integration research in Europe: A review and critique', in T. A. Aleinikoff \& D. Klusmeyer (eds.), Citizenship today: Global perspectives and practices. Washington, D.C.: Brookings Institute.

Favell, A. (1998), Philosophies of integration: Immigration and the idea of citizenship in France and Britain. Houndmills: Palgrave.

Fennema, M. (2002), Persstemmingen na 11 september. Amsterdam: IMES.

Fermin, A. (1997), Nederlandse politieke partijen over minderhedenbeleid 1977-1995. Amsterdam: Thesis Publishers.

Fischer, F. (2003), Reframing public policy: Discursive politics and deliberative practices. Oxford: Oxford University Press.

Fischer, F. (1993), 'Policy discourse and the politics of Washington think tanks', in F. A. Forester (ed.), The argumentative turn in policy analysis and planning, 21-42. London: Duke University Press.

Fischer, F. \& J. Forester (eds.) (1993), The argumentative turn in policy analysis and planning. London: Duke University Press.

Fortuyn, P. (1997), Tegen de islamisering van onze cultuur: Nederlandse identiteit als fundament. Utrecht: Bruna.

Freeman, G. (1995), 'Modes of immigration politics in liberal democratic states', International Migration Review 29 (4): 881-902. 
Gans, H. J. (1997), 'Toward a reconciliation of 'assimilation' and 'pluralism': The interplay of acculturation and ethnic retention', International Migration Review 31 (4): 875-892.

Gastelaars, M. (1985), Een geregeld leven: Sociologie en sociale politiek in Nederland 19251968. Amsterdam: SUA.

Geddes, A. (2005), 'Migration research and European integration: The construction and institutionalization of problems of Europe', in M. Bommes \& E. Morawska (eds.), International migration research: Constructions, omissions and interdisciplinarity, 265280. Aldershot: Ashgate.

Geddes, A. (2003), The politics of migration and immigration in Europe. London: Sage.

Geddes, A. \& V. Guiraudon (1996), 'The Europeanization of anti-discrimination in Britain and France', in C. Bertossi (ed.), European anti-discrimination and the politics of citizenship, 125-142. Houndmills: Palgrave.

Giddens, A. (1984), The constitution of society: Outline of the theory of structuration. Berkeley: University of California Press.

Gieryn, T. F. (1999), Cultural boundaries of science: Credibility on the line. Chicago: University of Chicago Press.

Gieryn, T. F. (1995), 'Boundaries of science', in S. Jasanoff, G. E. Markle, J. C. Petersen \& T. Pinch (eds.), Handbook of science and technology studies, 393-443. London: Thousand Oaks.

Gieryn, T. F. (1983), 'Boundary-work and the demarcation of science from non-science: Strains and interests in professional ideologies of scientists', American Sociological Review 4: 781-795.

Glaser, B. (1992). Basics of grounded theory analysis. Mill Valley: Sociology Press.

Glaser, B. G. \& A. L. Strauss (1967), The discovery of grounded theory: Strategies for qualitative research. New York: Aldine de Gruyter.

Goffman, E. (1974), Frame analysis: An essay on the organisation of experience. Cambridge: Harvard University Press.

Green, S. (2004), The politics of exclusion: Institutions and immigration policy in contemporary Germany. Manchester: Manchester University Press.

Groenendijk, K. (1990a), 'Verboden voor tukkers: Reacties op rellen tussen Italianen, Spanjaarden en Twentenaren in 1961', in B. A. T. Bovenkerk (ed.), Wetenschap en partijdigheid. Assen: Van Gorcum.

Groenendijk, K. (1990b), 'Verboden voor tukkers: Reacties op rellen tussen Italianen, Spanjaarden en Twentenaren in 1961', in F. Bovenkerk, F. Buijs \& H. Tromp (eds.), Wetenschap en partijdigheid, 55-96. Assen: Van Gorcum.

Guiraudon, V. (2006), 'Anti-discrimination policy', in P. Graziano \& M. Vink (eds.), Europeanization: New research agendas, 295-308. Basingstoke: Palgrave Macmillan.

Guiraudon, V. (2000a), 'European integration and migration policy: Vertical policy-making as venue shopping', Journal of Common Market Studies 38 (2): 251-271.

Guiraudon, V. (2000b), Les politiques d'immigration en Europe: Allemagne, France, PaysBas. Paris: L'Harmattan.

Guiraudon, V. (1997), 'Policy change behind gilded doors: Explaining the evolution of aliens' rights in France, Germany and the Netherlands, 1974-94', PhD thesis. Cambridge: Harvard University.

Gusfield, J. (1980), The culture of public problems: Drinking-driving and the symbolic order. Chicago: University of Chicago Press.

Gusfield, J. (1976), 'The literary rhetoric of science: Comedy and pathos in drinking driver research', American Sociological Review 41 (1): 16.

Guston, D. H. (2000), Between politics and science: Assuring the integrity and productivity of research. Cambridge: Cambridge University Press.

Haas, P. M. (1992), 'Introduction: Epistemic communities and international policy coordination', International organization 46 (1): 1-35. 
Hajer, M. A. (1995), The politics of environmental discourse: Ecological modernization and the policy process. Oxford: Oxford University Press.

Hajer, M. A. \& J. Uitermark (2007), 'Performing authority: Discursive politics after the assassination of Theo Van Gogh', Public Administration 86 (1): 5-19.

Halffman, W. (2005), 'Science-policy boundaries: National-styles?', Science \& Public Policy 32 (6): $457-467$.

Halffman, W. (2003), Boundaries of regulatory science: Eco/toxicology and aquatic hazards of chemicals in the US, England and the Netherlands. Boechout: Albatros.

Halffman, W. \& R. Hoppe (2006), 'Science/policy boundaries: A changing division of labour in Dutch expert policy advice', in S. Maasen \& P. Weingart (eds.), Scientific expertise and political decision making, 135-151. Dordrecht: Kluwer Academic Press.

Hall, P. A. (1993), 'Policy paradigms, social learning, and the state: The case of economic policymaking in Britain', Comparative Politics 25 (3): 275-296.

Hall, P. A. \& R. C. R. Taylor (1996), 'Political science and the three institutionalisms', Political Studies 44 (4): 936-957.

Hansen, R. (2003) 'Citizenship and integration in Europe', C. Joppke \& E. Morawska (eds.), Toward assimilation and citizenship: Immigrants in liberal nation-states, 263-282. New York: Palgrave Macmillan.

Heclo, H. (1978), 'Issue networks and the executive establishment', in A. King (ed.), American political system. Washington, D.C.: American Enterprise Institute for Public Policy Research.

Heclo, H. (1974), Modern social politics in Britain and Sweden. New Haven: Yale University Press.

Hess, D. J. (1997), Science studies: An advanced introduction. New York: New York University Press.

Hirsch-Ballin, E. M. H. (1997), 'Beleid boven de dagelijkse routine', in Scientific Council for Government Policy (ed.), Mosterd bij de maaltijd. The Hague: SDU.

Hirsch-Ballin, E. M. H. (1979), Publiekrecht en beleid: Fundamentele kwesties rondom het functioneren van de Wetenschappelijke Raad voor het Regeringsbeleid. Alphen aan den Rijn: Samson.

Hisschemöller, M. \& R. Hoppe (1995), 'Coping with intractable controversies: The case for problem structuring in policy design and analysis', Knowledge for policy 4 (8): 40-70.

Hoffmann-Nowotny, H. J. (1973), Soziologie des Fremdarbeiterproblems: Eine theoretische und empirische Analyse am Beispiel der Schweiz. Stuttgart: Ferdinand Enke Verlag.

Holsteyn, J. J. M. \& G. A. Irwin (2003), 'A new kid on the block: Pim Fortuyn and the Dutch Parliamentary Election of May 2002', British Elections and Parties Review 13: 29-46.

Hoppe, R. (2005), 'Rethinking the science-policy nexus: From knowledge utilization and science technology studies to types of boundary arrangements', Poiesis \& Praxis: International Journal of Technology Assessment and Ethics of Science 3 (3): 199-215.

Hoppe, R. (1999), 'Policy analysis, science and politics: From "speaking truth to power" to "making sense together", Science and Public Policy 26 (3): 201-201.

Hoppe, R. (1993), 'Political judgement and the policy cycle: The case of ethnicity policy arguments in the Netherlands', in F. Fischer \& J. Forester (eds.), The argumentative turn in policy analysis and planning. London: Duke University Press.

Ireland, P (1994), The policy challenge of ethnic diversity: Immigrant politics in France and Switzerland. Cambridge, Mass.: Harvard University Press.

Jacobson, D. (1996), Rights across borders: Immigration and the decline of citizenship. Baltimore: The John Hopkins Press.

Jasanoff, S. (2004), States of knowledge: The co-production of science and social order. New York: Routledge.

Jasanoff, S. (1990), The fifth branch: Science advisers as policymakers. London: Cambridge University Press. 
Joppke, C. (1999), Immigration and the nation-state: The United States, Germany and Great Britain. Oxford: Oxford University Press.

King, G., R. O. Keohane, \& S. Verba (1994), Designing social inquiry: Scientific inference in qualitative research. Princeton: Princeton University Press.

Kingdon, J. W. (1995), Agendas, alternative, and public policies. Amsterdam: Addison Wesley.

Kivisto, P. (2001), 'Theorizing transnational immigration: A critical review of current efforts', Ethnic and Racial Studies 24 (4): 549-577.

Knorr-Cetina, K. D. (1995), 'Laboratory studies: The cultural approach', in J. E. Al (ed.), Handbook of science and technology studies, 140-166. Thousand Oaks: Sage.

Köbben, A. J. F. \& H. Tromp (1999), De onwelkome boodschap of hoe de vrijheid van wetenschap bedreigd wordt. Amsterdam: Mets.

Köbben, A. J. F. (1986), “'Etnische minderheden”: De lusten en lasten van een term', in D. Beus \& V. Doorn (eds.), De geconstrueerde samenleving. Meppel: Boom.

Köbben, A. J. F. (1983), De zaakwaarnemer. Deventer: Van Loghum Slaterus.

Köbben, A. J. F. (1980), 'Het heilig vuur: Over moeilijkheden en mogelijkheden bij onderzoek inzake minderheden', inaugural speech. Leiden University.

Köbben, A. J. F. (1979), 'De gijzelingsakties van zuidmolukkers en hun effekten op de samenleving', Transaktie (2): 147-154.

Koolen, G. M. J. M. (2003), Integratiebeleid in de tijd: Analyse van de ontwikkeling van het gecoordineerde minderheden-/integratiebeleid met betrekking tot etnische minderheden, 1945-2003. The Hague: Department of Home Affairs.

Koolen, G. M. J. M. \& S. G. Tempelman (2003), Integratiebeleid in de tijd: Feitelijk overzicht van de ontwikkeling van het gecoordineerde minderheden-/integratiebeleid met betrekking tot etnische minderheden. The Hague: Department of Home Affairs.

Koopmans, R. (2003), 'Good intentions sometimes make bad policy: A comparison of Dutch and German integration policies', in R. Cuperus, K. Duffek \& J. Kandel (eds.), The challenge of diversity. Innsbruck: Studienverlag.

Koopmans, R. (2003), 'Uitvluchten kan niet meer...: Repliek op Böcker en Thränhardt', Migrantenstudies 19 (1): 45-56.

Koopmans, R. (2002), 'Zachte heelmeesters...: Een vergelijking van de resultaten van het Nederlandse en het Duitse integratiebeleid en wat de WRR daaruit niet concludeert', Migrantenstudies 18: 87-92.

Koopmans, R., Statham, P., Giugni, M. \& F. Passy (2005), Contested citizenship: Immigration and cultural diversity in Europe. Minneapolis: University of Minnesota Press.

Koopmans, R. \& S. Statham (2000), 'Migration and ethnic relations as a field of political contention: An opportunity structure approach', in R. Koopmans \& S. Statham (eds.), Challenging immigration and ethnic relations politics, 13-56. Oxford: Oxford University Press.

Kuhn, T. S. (1964), The structure of scientific revolutions. Chicago: University of Chicago Press.

Kymlicka, W. (1995), Multicultural citizenship. Oxford: Oxford University Press.

Latour, B. (1993), We have never been modern. Cambridge: Harvard University Press.

Latour, B. (1988), The pasteurization of France. Cambridge: Harvard University Press.

Latour, B. \& S. Woolgar (1986), Laboratory life: The construction of scientific facts. Princeton: Princeton University Press.

Lauman, E. O. \& D. Knoke (1987), The Organizational state: Social choice in national policy domains. London: University of Wisconsin Press.

Lavenex, S. (2005), 'National frames in migration research: The tacit political agenda', in M. Bommes \& E. Morawska (eds.), International migration research: Constructions, omissions and interdisciplinarity, 243-264. Aldershot: Ashgate. 
Leibfried, S. (2000), 'National welfare states, European integration and globalization: A perspective for the next century', Social Policy \& Administration 34 (1): 44-63.

Leibfried, S. (1992) 'Towards a European welfare state? On integrating poverty regimes into the European Community', in Z. Ferge \& J. E. Kolberg (eds.), Social Policy in a Changing Europe, 245-279. Frankfurt am Main: Campus Verlag.

Lijphart, A. (1968), The politics of accommodation: Pluralism and democracy in the Netherlands. Berkeley: University of California Press

Lucassen, L. \& A. J. F. Köbben (1992), Het partiële gelijk: Controverses over het onderwijs in eigen taal en cultuur en de rol daarbij van beleid en wetenschap. Amsterdam: Swets en Zeitlinger.

Lukes, S. (1974), Power: A radical view. London: Macmillan.

Maasen, S. \& P. Weingart (2000), Metaphors and the dynamics of knowledge. London: Routledge.

Mariën, M. H. (1992), Maatschappelijk debat integratie: Discussie minderheden in de pers. The Hague: Department of Home Affairs.

Mayeur, L. (2006), 'La recherche sur l'immigration en France du point de vue de la recherche publique', Paper presented at Migrinter conference, University of Poitiers, 5-7 July 2006.

Meloen, J. D. \& J. Veenman (1990), Het is maar de vraag ...: Onderzoek naar responseffecten bij minderhedensurveys. Lelystad: Vermande.

Meurs, P. \& D. Broeders (2001), 'Nederland als immigratiesamenleving', Migrantenstudies 18 (2): 64-77.

Miles, R. (1987), 'Recent Marxist theories of nationalism and the issue of racism', The British Journal of Sociology 38 (1): 24-43.

Miller, C. A. (2001), 'Hybrid management: Boundary organizations, science policy, and environmental governance in the climate regime', Science, Technology and Human Values 26 (4): 478-500.

Molleman, H. (2003), 'Het minderhedenbeleid in retrospectief', Socialisme \& Democratie 60 (1-2): 62-66.

Molleman, H. (1978), 'Culturele minderheden en overheidsbeleid', Socialisme en Democratie 7/8, 328-343.

Mulkay, G. A. (1984), Opening Pandora's box: A sociological analysis of scientists' discourse. Cambridge: Cambridge University Press.

Muus, P. \& R. Penninx (1989), 'Kanttekeningen bij de wetenschappelijkheid van het WRRrapport "Allochtonenbeleid", Migrantenstudies 5 (4): 2-53.

Nelkin, D. (1979), Controversy: Politics of technical decisions. Beverly Hills: Sage.

Nowotny, H., P. Scott \& M. Gibbons (2001), Re-thinking science: Knowledge and the public in an age of uncertainty. London: Polity Press.

Outhwaite, W. (1996), The Habermas reader. London: Polity Press.

Parekh, B. (2000), Rethinking multiculturalism: Cultural diversity and political theory. Houndmills: Palgrave.

Park, R. E. (1928), 'Human migration and the marginal man', American Journal of Sociology 33: 881-893.

Park, R. E. \& E. W. Burgess (1921), Introduction to the science of sociology. Chicago: University of Chicago Press.

Parsons, W. (1995), Public policy: An introduction to the theory and practice of policy analysis. Cheltenham: Edward Elgar.

Pellikaan, H. \& M. Trappenburg (eds.) (2003), Politiek in de multiculturele samenleving. Amsterdam: Boom.

Penninx, R. (2005), 'Bridges between research and policy? The case of post-war immigration and integration policies in the Netherlands', International Journal on Multicultural Studies 7 (1): 33-48. 
Penninx, R. (1992), Wie betaalt en wie bepaalt? Onderzoeksbeleid van de overheid m.b.t. minderheden en de invloed van onderzoek op beleid. The Hague: Department of Home Affairs.

Penninx, R. (1988a), Minderheidsvorming en emancipatie: Balans van kennisverwerving ten aanzien van immigranten en woonwagenbewoners, 1967-1987. Alphen aan den Rijn: Samsom.

Penninx, R. (1988b), Wie betaalt, bepaalt? De ontwikkeling en programmering van onderzoek naar migranten, etnische minderheden en woonwagenbewoners 1955-1985, met speciale aandacht voor de rol van de overheid. Amsterdam: Instituut voor Sociale Geografie.

Penninx, R. (1979), 'Naar een algemeen etnisch minderhedenbeleid?', in S. C. F. G. Policy (ed.), Etnische minderheden. The Hague: Staatsuitgeverij.

Penninx, R., B. Garcés-Mascareñas \& P. Scholten (2005), 'Policymaking related to immigration and integration: A review of the literature of the Dutch case', IMISCOE Working Paper 8, available at http://imiscoe.socsci.uva.nl/publications/workingpapers/index.html.

Penninx, R., J. Schoorl, \& C. Van Praag (1993), The impact of international migration on receiving countries: The case of the Netherlands. Amsterdam: Swets \& Zeitlinger.

Pierson, P. (1994), Dismantling the Welfare State? Reagan, Thatcher and the politics of retrenchment. Cambridge: Cambridge University Press.

Poppelaars, C. \& P. Scholten (2008). 'Two worlds apart: The divergence of national and local integration policies in the Netherlands', Administration \& Society 40 (4).

Popper, K. R. (1962), Conjectures and refutations: The growth of scientific knowledge. New York: Harper and Grow.

Powell, W. W. \& P. J. DiMaggio (eds.) (1991), The new institutionalism in organizational analysis. Chicago: University of Chicago Press.

Pralle, S. B. (2003), 'Venue shopping, political strategy, and policy change: The internationalization of Canadian forest advocacy', Journal for Public Policy 23 (3): 233-260.

Prins, B. (2002a), Dol op drama: Een reconstructie van het publieke debat naar aanleiding van Paul Scheffers 'Het multiculturele drama'. Amsterdam: De Balie.

Prins, B. (2002b), 'Het lef om taboes te doorbreken: Nieuw realisme in het Nederlandse discours over multiculturalisme', Migrantenstudies 18 (4): 241-254.

Prins, B. (2000), Voorbij de onschuld: Het debat over de multiculturele samenleving. Amsterdam: Van Gennep.

Prins, B. (1997), The standpoint in question: Situated knowledges and the Dutch minorities discourse. Utrecht: Utrecht University.

Radin, B. A. (2000), Beyond Machiavelli: Policy analysis comes of age. Washington, D.C.: Georgetown University Press.

Ratcliffe, P. (2001), 'Race and ethnicity research in Britain: Key ethical and political considerations', in P. Ratcliffe (ed.), The politics of social science research: Race, ethnicity and social change, 109-136. New York: Palgrave.

Rath, J. (2001), 'Research on immigrant ethnic minorities in the Netherlands', in P. Ratcliffe (ed.), The politics of social science research: Race, ethnicity and social change, 160-176. New York: Palgrave.

Rath, J. (1991), Minorisering: De sociale constructie van 'etnische minderheden'. Amsterdam: SUA.

Rein, M. (2008), 'Lost frames', closing speech at IMISCOE/IGS conference Research-Policy Dialogues on Migration and Integration in Europe, University of Twente, Enschede, the Netherlands, 22-23 May 2008.

Rein, M. (1986), Frame-reflective policy discourse. Leiden: Leiden University Press.

Rein, M. \& D. Schön (1996), 'Frame-critical policy analysis and frame-reflective policy practice', Knowledge and Policy: The International Journal of Knowledge Transfer and Utilization 9 (1): 85-104. 
Rein, M., \& D. Schön (1994), Frame reflection: Toward the resolution of intractable policy controversies. New York: Basic Books.

Rein, M. \& D. Schön (1993), 'Reframing policy discourse', in F. Fischer \& J. Forester (eds.), The argumentative turn in policy analysis and planning. London: Duke University Press.

Rittel, H. W. J. \& M. M. Webber (1973), 'Dilemmas in a general theory of planning', Policy Sciences 4: 155-169.

Rose, E. J. B. (1969), Colour and citizenship: A report on British race relations. Oxford: Oxford University Press.

Rubington, E. \& M. S. Weinberg (1995), The study of social problems: Seven perspectives. Oxford: Oxford University Press.

Sabatier, P. A. (1987), 'Knowledge, policy-oriented learning and policy change', Knowledge: Creation, Diffusion, Utilisation 8: 649-692.

Sabatier, P. A. \& H. C. Jenkins-Smith (1999), 'The advocacy coalition framework: An assessment', in P.A. Sabatier (ed.), Theories of the policy process: Theoretical lenses on public policy. Boulder: Westview Press.

Sabatier, P. A. \& H. C. Jenkins-Smith (1995), Policy change and learning: An advocacy coalition approach. Boulder: Westview Press.

Sabbagh, S. \& S. Peer (2008), 'French color-blindness in perspective: The controversy over "statistiques ethniques", French Politics, Culture \& Society 26 (1): 1-8.

Saggar, S. (1992), Race and public policy. Aldershot: Avebury.

Salter, L. \& E. Levy (1988), Mandated science: science and scientists in the making of standards. Dordrecht: Kluwer Academic Publishers.

Sassen, S. (1998), 'The de facto transnationalisation of immigration policy', in C. Joppke (ed.), Challenge to the nation-state. Oxford: Oxford University Press.

Schain, M. (1999), 'Minorities and immigrant incorporation in France', in C. Joppke \& S. Lukes (eds.), Multicultural questions. Oxford: Oxford University Press.

Schain, M. (1995), 'Policy and policy making in France and the US: Models of incorporation and the dynamics of change', Modern and Contemporary France 3 (4).

Schattschneider, E. E. (1960), The semi-sovereign people: A realist's view of democracy in America. New York: Holt, Rinehart \& Winston.

Schnabel, P. (1998), De multiculturele illusie: Een pleidooi voor aanpassing en assimilatie. Utrecht: FORUM.

Schnapper, D. (1991) La France de l'intégration: Sociologie de la nation en 1990. Paris: Gallimard.

Schneider, A. L. \& H. Ingram (1997), Policy design for democracy. Kansas: University of Kansas Press.

Schneider, A. L. \& H. Ingram (1993), 'Social construction of target populations: Implications for politics and policy', The American Political Science Review 87 (2): 334-347.

Schneider, J. (2007), 'Modernes regieren und konsens? Regierungskommissionen im politikprozess, untersucht am beispiel der unabhängigen kommission "Zuwanderung", $\mathrm{PhD}$ thesis, Justus-Liebig-Universität Giessen.

Scholten, P. (2008), 'Constructing immigrant policies: Research-policy relations and immigrant integration in the Netherlands, 1970-2004', PhD thesis, University of Twente.

Scholten, P. \& A. Timmermans (2004), 'Doorbraken en zachte landingen in het Nederlandse immigrantenbeleid: Een theoretische analyse van beleidsdynamiek', Beleidswetenschap 18 (1): 3-30.

Scholten, P. \& F. Van Nispen (2008), 'Building bridges across frames? A meta-evaluation of Dutch immigrant integration policy', Journal of Public Policy 28 (2).

Schuster, J. (1999), Poortwachters over immigranten: Het debat over immigratie in het naoorlogse Groot-Brittannië en Nederland. Amsterdam: Het Spinhuis.

Scientific Council for Government Policy (2001a), Naar een Europabrede unie. The Hague: SDU. 
Scientific Council for Government Policy (2001b), The Netherlands as an immigration society. The Hague: SDU.

Scientific Council for Government Policy (1992), Evaluatie vierde raadsperiode (1987-1992). The Hague: WRR.

Scientific Council for Government Policy (1990), Werk in perspectief. The Hague: SDU.

Scientific Council for Government Policy (1989), Immigrant policy. The Hague: SDU.

Scientific Council for Government Policy (1988), Working programme of fourth council (1988-1992). The Hague: WRR.

Scientific Council for Government Policy (1987a), Activerend arbeidsmarktbeleid. The Hague: SDU.

Scientific Council for Government Policy (1987b), Verslag en evaluatie van de derde raadsperiode 1983-1987. The Hague: WRR.

Scientific Council for Government Policy (1979), Ethnic minorities. The Hague: SDU.

Scientific Council for Government Policy (1977), Verslag eerste raadsperiode 1972-1977. The Hague: WRR.

Shapin, S. (1992), 'Discipline and bounding: The history and sociology of science as seen through the externalism-internalism debate', History of Science 30 (90): 333-369.

Shapin, S. \& S. Schaffer (1985), The Leviathan and the air-pump: Hobbes, Boyle and the experimental life. Princeton: Princeton University Press.

Singh, G. (2004), 'Multiculturalism in contemporary Britain: Community cohesion, urban riots and the "Leicester Model", in J. Rex \& G. Singh (eds.), Governance in multicultural societies, 56-81. Aldershot, Ashgate.

Small, S. \& J. Solomos (2006), 'Race, immigration and politics in Britain: Changing policy agendas and conceptual paradigms 1940s-2000s', International Journal of Comparative Sociology 8 (47): 235-257.

Smeets, H. \& J. Veenman (2000), 'More and more at home: Three generations of Moluccans in the Netherlands', in H. Vermeulen \& R. Penninx (eds.), Immigrant integration: The Dutch case. Amsterdam: Het Spinhuis.

Snel, E. (2003a), De vermeende kloof tussen culturen. Enschede: Oratie, University of Twente.

Snel, E. (2003b), 'Integratie is niet mislukt', Socialisme \& Democratie 10 (1/2): 30-38.

Snel, E. \& G. Engbersen (2002), 'Op weg naar transnationaal burgerschap: De schuivende panelen van internationale migratie', in F. Becker, W. Van Hennekeler, M. Sie Dhian Ho \& B. Tromp (eds.), Transnationaal burgerschap: Immigratie en integratie. Amsterdam: De Arbeiderspers.

Snel, E. \& P. Scholten (2005), 'Van gastarbeiders tot het multiculturele drama: Integratie als hardnekkig beleidsprobleem', in M. Arentsen \& W. Trommel (eds.), Moderniteit en overheidsbeleid: Hardnekkige beleidsproblemen en hun oorzaken, 155-183. Bussum: Coutinho.

Social and Cultural Planning Office (2006a), Turken in Nederland en Duitsland: De arbeidsmarktpositie vergeleken. The Hague: SCP.

Social and Cultural Planning Office (2006b), Werkprogramma 2006-2007. The Hague: SCP.

Social and Cultural Planning Office (2005), Jaarverslag 2004. The Hague: SCP.

Social and Cultural Planning Office (2003), Rapportage minderheden. The Hague: SCP.

Social and Cultural Planning Office (2002), Werkprogramma 2002-2003. The Hague: SCP.

Social and Cultural Planning Office (2001), Rapportage minderheden. The Hague: SCP.

Social and Cultural Planning Office (1998), Sociaal en cultureel rapport 1998: 25 jaar sociale verandering. The Hague: SCP.

Social and Cultural Planning Office (1986), Social en cultureel rapport. The Hague: SCP.

Soysal, Y. N. (1994), Limits of citizenship: Migrants and postnational membership in Europe. Chicago: Chicago University Press. 
Spector, M. \& J. I. Kitsuse (1977), Constructing social problems. Menlo Park, Cal.: Cummings.

Star, S. L. \& J. R. Griesemer (1989), 'Institutional ecology, "translations" and boundary objects: Amateurs and professionals in Berkeley's Museum of Vertebrate Zoology, 19071939', Social Studies of Science 19: 387-420.

Statistiek, N. S. V. (1971), De buitenlandse arbeider in Nederland. The Hague: SDU.

Stone, D. (2002 [1988]), Policy paradox: The art of political decision making. London: WW Norton \& Company.

Stone, D. (1998), Capturing the political imagination: Think tanks and the policy process. London: Cass.

Stone, D. (1989), 'Causal stories and the formation of policy agendas', Political Science Quarterly 104 (2): 281-300.

Stone, D. \& M. Garnett (1998), 'Introduction: Think tanks, policy advice and governance', in H. A. G. Stone (ed.), Think tanks across nations: A comparative approach, 1-40. Manchester: Manchester University Press.

Surie, H. G. (1971), 'De gerepatrieerden', in H. Verwey-Jonker (ed.), Allochtonen in Nederland. The Hague: Staatsuitgeverij.

Taylor, C. (1992), Multiculturalism and the politics of recognition. Princeton: Princeton University Press.

Tilly, C., G. Noiriel \& G. De Laforcade (1996), The French melting pot: Immigration, citizenship and national identity. Minneapolis: University of Minnesota Press.

Timmermans, A. \& P. Scholten (2006), 'The political flow of wisdom: Science institutions as policy venues in the Netherlands', Journal of European Public Policy 13 (7): 1104-1118.

Tinnemans, W. (1994), Een gouden armband:Een geschiedenis van mediterrane immigranten in Nederland (1945-1994). Utrecht: NCB.

Tribalat, M. (1996), De l'immigration à l'assimilation: Enquête sur les populations d'origine étrangère en France. Paris: La découverte.

Turner, B. S. (2006), 'British sociology and public intellectuals: Consumer society and imperial decline', The British Journal of Sociology 57 (2): 169-188.

Urbanus, R. (1983), Reacties op de ontwerp-minderhedennota: Een analyse van reacties op de ontwerp-minderhedennota. Leiden: COMT.

Van Amersfoort, J. M. M. (2001), Transnationalisme, moderne diaspora's en sociale cohesie. Amsterdam: IMES.

Van Amersfoort, J. M. M. (1991), 'Van gerepatrieerdenzorg tot allochtonenbeleid: Wetenschap en beleid met betrekking tot immigranten in Nederland', De Sociologische Gids 9 (1): 2436.

Van Amersfoort, J. M. M. (1984), 'Migratie-onderzoek, overheidsfinanciering en beleid: Aantekeningen van een participant', Grafiet 1983 (4): 130-154.

Van Amersfoort, J. M. M. (1974), Immigratie en minderheidsvorming: Een analyse van de Nederlandse situatie 1945-1973. Alphen aan den Rijn: Samson.

Van Boxtel, R. H. M. L. (2000), 'Integratie: een gezamenlijke verantwoordelijkheid', in J. E. Overdijk-Francis \& H. M. A. G. Smeets (eds.), Bij nader inzien: Het integratiedebat op afstand bekeken. Houten/Diegem: Bohn Stafleu van Loghum.

Van den Berg-Eldering, L. (1978), Marokkaanse gezinnen in Nederland. Alphen aan den Rijn: Samson.

Van der Veen, R. \& W. Trommel (1999), De herverdeelde samenleving: De ontwikkeling en herziening van de Nederlandse verzorgingsstaat. Amsterdam: Amsterdam University Press.

Van der Zwan, A. \& H. Entzinger (1994), Beleidsopvolging minderhedendebat: Advies in opdracht van de minister van binnenlandse zaken. The Hague: Ministry of Foreign Affairs.

Van Eeten, M. J. G. (1999), 'Dialogues of the deaf': On science in policy controversies', Science and Public Policy 26 (3): 185-192. 
Van Hoesel, P. H. M. (1984), Programmering van sociaal wetenschappelijk beleidsonderzoek. Leiden: Lisbon.

Van Kuik, F. (1986), 'Zeven jaar minderhedenbeleid', Bestuur 5: 118-123.

Van Praag, C. (2003), Berichten uit het vyvarium: Columns van K. Vyvary met een inleiding van Carlo van Praag. The Hague: SCP.

Van Praag, C. (1987), 'Onderzoek naar etnische minderheden in Nederland: Een signalement', De Sociologische Gids 34 (3): 159-175.

Van Praag, C. (1986), 'Minderheden voor en na de nota', Migrantenstudies 18 (4): 2-53.

Van Praag, C. (1984), Evenredigheid en toegankelijkheid sociale positie en voorzieningengebruik van etnische minderheden. The Hague: SCP.

Van Putten, N. (1990), 'De adviescommissie onderzoek minderheden: College met gezag', in F. Bovenkerk, F. Buijs \& H. Tromp (eds.), Wetenschap en partijdigheid, 357-372. Assen: Van Gorcum.

Van Thijn, E. (1994), Retour Den Haag: Dagboek van een minister. Amsterdam: Van Gennep.

Van Thijn, E. (1985), Minderhedenbeleid in drievoud. The Hague: Department of Home Affairs.

Vasta, E. \& V. Vuddamalay (2006), International migration and the social sciences. Hampshire: Macmillan.

Veenman, J. (2001), Molukse jongeren in Nederland: Integratie met de rem erop. Assen: Van Gorcum.

Vermeulen, H. \& R. Penninx (eds.) (2000), Immigrant Integration: The Dutch Case. Amsterdam: Het Spinhuis.

Vermeulen, H. \& R. Penninx (eds.) (1994), Het democratisch ongeduld: De emancipatie en integratie van zes doelgroepen van het minderhedenbeleid. Amsterdam: Het Spinhuis.

Vertovec, S. (2001), 'Transnational challenges to the "new" multiculturalism', paper presented at the ASA Conference, Sussex, 30 March - 2 April 2001.

Verwey-Jonker, H. (1971), Allochtonen in Nederland. The Hague: Staatsuitgeverij.

VWJ (2004), 'Bronnenonderzoek: Tijdelijke commissie onderzoek integratiebeleid', research report by the Verwey-Jonker Institute. The Hague: SDU.

Vink, M. (2007), 'Dutch multiculturalism: Beyond the pillarisation myth', Political Studies Review 5 (3): 337-350.

Vuijsje, H. (1986), Vermoorde onschuld: Etnisch verschil als Hollands taboe. Amsterdam: Bert Bakker.

Wansink, H. (2004), De erfenis van Fortuyn: De Nederlandse democratie na de opstand van de kiezers. Amsterdam: Uitgeverij Meulenhoff.

Weaver, K. (1989), 'The changing world of think tanks', Political Science and Politics 22 (3): 563-578.

Weber, M. (1988), Gesamtliche politische schriften. Tübingen: Mohr Schiebeck.

Weber, M., D. Owen, T. Strong \& R. Livingstone (eds.) (2004). The vocation lectures: Science as a vocation, politics as a vocation. Indianapolis: Hackett Pub Co.

Weil, P. (1991), La France et ses étrangers: L'aventure d'une politique de l'immigration de 1938 à nos jours. Paris: Calmann-Lévy.

Weingart, P. (1999), 'Scientific expertise and political accountability: Paradoxes of science in politics', Science and Public Policy 26 (3): 151-162.

Weiss, C. H. (1991), 'Policy research: Data, ideas, or arguments?', in P. Wagner (ed.), Social sciences and modern states: National experiences and theoretical crossroads, 307-332. Cambridge: Cambridge University Press.

Weiss, C. H. (1977), 'Research for policy's sake: The enlightenment function of social science research', Policy Analysis 3 (4): 531.

Weiss, R. (1994), Learning from strangers: The art and method of qualitative interview studies. New York: The Free Press. 
Wentholt, R. (ed.) (1967), Buitenlandse arbeiders in Nederland: Een veelzijdige benadering van een complex vraagstuk. Leiden: Spruyt, Van Mantgem \& De Dres NV.

Werdmölder, H. (2003), 'Politiek correct denken: Een reconstructie achteraf', Socialisme \& Democratie 59 (2): 35-50.

Werdmölder, H. (1992), 'De armoede van het minderhedenonderzoek', Socialisme \& Democratie 49 (2): 53-76.

Wieviorka, M. (1995), The arena of racism. London: Sage.

Wildavsky, A. (1979), The art and craft of policy analysis: Speaking truth to power. London: Macmillian.

Wittrock, B. (1991), 'Social knowledge and public policy: Eight models of interaction', in P. Wagner (ed.), Social sciences and modern states: National experiences and theoretical crossroads, 28-85. Cambridge: Cambridge University Press.

Yanow, D. (2000), Conducting interpretive policy analysis. London: Sage.

Yanow, D. (1996), How does a policy mean? Interpreting policy and organizational actions. Washington, D.C.: Georgetown University Press.

Yin, R. K. (1994), Case study research: Design and methods, 2nd ed. London: Sage. 
Other IMISCOE titles

\section{IMISCOE Research}

Rinus Penninx, Maria Berger, Karen Kraal, Eds.

The Dynamics of International Migration and Settlement in Europe:

A State of the Art 2006 (ISBN 978905356866 8)

(originally appearing in IMISCOE Joint Studies)

Leo Lucassen, David Feldman, Jochen Oltmer, Eds.

Paths of Integration: Migrants in Western Europe (1880-2004)

2006 (ISBN 978905356883 5)

Rainer Bauböck, Eva Ersbøll, Kees Groenendijk, Harald Waldrauch, Eds. Acquisition and Loss of Nationality: Policies and Trends in 15 European Countries, Volume 1: Comparative Analyses

2006 (ISBN 978905356920 7)

Rainer Bauböck, Eva Ersbøll, Kees Groenendijk, Harald Waldrauch, Eds. Acquisition and Loss of Nationality: Policies and Trends in 15 European Countries, Volume 2: Country Analyses

2006 (ISBN 978905356921 4)

Rainer Bauböck, Bernhard Perchinig, Wiebke Sievers, Eds.

Citizenship Policies in the New Europe

2007 (ISBN 978905356922 1)

Veit Bader

Secularism or Democracy? Associational Governance of Religious

Diversity

2007 (ISBN 978905356999 3)

Holger Kolb, Henrik Egbert, Eds.

Migrants and Markets: Perspectives from Economics and the Other Social Sciences

2008 (ISNB 978905356684 8)

Ralph Grillo, Ed.

The Family in Question: Immigrant and Ethnic Minorities in Multicultural Europe

2008 (ISBN 978905356869 9) 
Corrado Bonifazi, Marek Okólski, Jeannette Schoorl, Patrick Simon, Eds. International Migration in Europe: New Trends and New Methods of Analysis

2008 (ISBN 978905356894 1)

Maurice Crul, Liesbeth Heering, Eds.

The Position of the Turkish and Moroccan Second Generation in Amsterdam and Rotterdam: The TIES Study in the Netherlands 2008 (ISBN 978908964061 1)

Marlou Schrover, Joanne van der Leun, Leo Lucassen, Chris Quispel, Eds. Illegal Migration and Gender in a Global and Historical Perspective 2008 (ISBN 978908964047 5)

Gianluca P. Parolin

Citizenship in the Arab World: Kin, Religion and Nation-State 2009 (ISBN 978908964045 1)

Rainer Bauböck, Bernhard Perchinig, Wiebke Sievers, Eds.

Citizenship Policies in the New Europe: Expanded and Updated Edition 2009 (ISBN 978908964108 3)

Cédric Audebert, Mohamed Kamel Dorai, Eds.

Migration in a Globalised World: New Research Issues and Prospects 2010 (ISBN 978908964 1571)

Richard Black, Godfried Engbersen, Marek Okólski, Cristina Pantîru, Eds. A Continent Moving West? EU Enlargement and Labour Migration from Central and Eastern Europe 2010 (ISBN 978908964156 4)

Charles Westin, José Bastos, Janine Dahinden, Pedro Góis, Eds. Identity Processes and Dynamics in Multi-Ethnic Europe 2010 (ISBN 978908964046 8)

Rainer Bauböck, Thomas Faist, Eds.

Diaspora and Transnationalism: Concepts, Theories and Methods 2010 (ISBN 978908964238 7)

Liza Mügge

Beyond Dutch Borders: Transnational Politics among Colonial Migrants, Guest Workers and the Second Generation 2010 (ISBN 978908964244 8) 
Blanca Garcés-Mascareñas

Labour Migration in Malaysia and Spain: Markets, Citizenship and Rights 2011 (ISBN 978908964286 8)

Albert Kraler, Eleonore Kofman, Martin Kohli, Camille Schmoll, Eds. Gender, Generations and the Family in International Migration 2011 (ISBN 978908964285 1)

Michael Bommes, Giuseppe Sciortino, Eds.

Foggy Social Structures: Irregular Migration, European Labour Markets and the Welfare State

2011 (ISBN 9789089643414 )

Bram Lancee

Immigrant Performance in the Labour Market: Bonding and Bridging Social Capital

2011 (ISBN 978908964357 5)

Julie Vullnetari

Albania on the Move: Links between Internal and International Migration 2011 (ISBN 978908964355 1)

\section{IMISCOE Reports}

Rainer Bauböck, Ed.

Migration and Citizenship: Legal Status, Rights and Political Participation 2006 (ISBN 978905356888 0)

Michael Jandl, Ed.

Innovative Concepts for Alternative Migration Policies: Ten Innovative Approaches to the Challenges of Migration in the 21st Century 2007 (ISBN 978905356990 0)

Jeroen Doomernik, Michael Jandl, Eds.

Modes of Migration Regulation and Control in Europe 2008 (ISBN 978905356689 3)

Michael Jandl, Christina Hollomey, Sandra Gendera, Anna Stepien, Veronika Bilger

Migration and Irregular Work In Austria: A Case Study of the Structure and Dynamics of Irregular Foreign Employment in Europe at the Beginning of the $21^{\text {st }}$ Century

2008 (ISBN 978908964053 6) 
Heinz Fassmann, Ursula Reeger, Wiebke Sievers, Eds.

Statistics and Reality: Concepts and Measurements of Migration in Europe 2009 (ISBN 978908964052 9)

Karen Kraal, Judith Roosblad, John Wrench, Eds.

Equal Opportunities and Ethnic Inequality in European Labour Markets Discrimination, Gender and Policies of Diversity

2009 (ISBN 978908964126 7)

Tiziana Caponio, Maren Borkert, Eds.

The Local Dimension of Migration Policymaking

2010 (ISBN 978908964232 5)

Raivo Vetik, Jelena Helemäe, Eds.

The Russian Second Generation in Tallinn and Kohtla-Järve: The TIES

Study in Estonia

2010 (ISBN 978908964250 9)

\section{IMISCOE Dissertations}

Panos Arion Hatziprokopiou

Globalisation, Migration and Socio-Economic Change in Contemporary Greece: Processes of Social Incorporation of Balkan Immigrants in Thessaloniki

2006 (ISBN 978905356873 6)

Floris Vermeulen

The Immigrant Organising Process: Turkish Organisations in Amsterdam and Berlin and Surinamese Organisations in Amsterdam, 1960-2000 2006 (ISBN 978905356875 0)

Anastasia Christou

Narratives of Place, Culture and Identity: Second-Generation

Greek-Americans Return 'Home'

2006 (ISBN 978905356878 1)

Katja Rušinović

Dynamic Entrepreneurship: First and Second-Generation Immigrant

Entrepreneurs in Dutch Cities

2006 (ISBN 978905356972 6) 
Ilse van Liempt

Navigating Borders: Inside Perspectives on the Process of Human

Smuggling into the Netherlands

2007 (ISBN 978905356930 6)

Myriam Cherti

Paradoxes of Social Capital: A Multi-Generational Study of Moroccans

in London

2008 (ISBN 978905356032 7)

Marc Helbling

Practising Citizenship and Heterogeneous Nationhood: Naturalisations in Swiss Municipalities

2008 (ISBN 978908964034 5)

Jérôme Jamin

L'imaginaire du complot: Discours d'extrême droite en France et aux Etats-Unis

2009 (ISBN 978908964048 2)

Inge Van Nieuwenhuyze

Getting by in Europe's Urban Labour Markets: Senegambian Migrants' Strategies for Survival, Documentation and Mobility

2009 (ISBN 978908964050 5)

Nayla Moukarbel

Sri Lankan Housemaids in Lebanon: A Case of 'Symbolic Violence' and 'Every Day Forms of Resistance'

2009 (ISBN 978908964051 2)

John Davies

'My Name Is Not Natasha': How Albanian Women in France Use

Trafficking to Overcome Social Exclusion (1998-2001)

2009 (ISBN 978905356707 4)

Dennis Broeders

Breaking Down Anonymity: Digital Surveillance of Irregular Migrants in Germany and the Netherlands

2009 (ISBN 978908964159 5)

Arjen Leerkes

Illegal Residence and Public Safety in the Netherlands

2009 (ISBN 978908964049 9) 
Jennifer Leigh McGarrigle

Understanding Processes of Ethnic Concentration and Dispersal:

South Asian Residential Preferences in Glasgow

2009 (ISBN 978905356671 8)

João Sardinha

Immigrant Associations, Integration and Identity: Angolan, Brazilian

and Eastern European Communities in Portugal

2009 (ISBN 978908964036 9)

Elaine Bauer

The Creolisation of London Kinship: Mixed African-Caribbean and White British Extended Families, 1950-2003

2010 (ISBN 978908964235 6)

Nahikari Irastorza

Born Entrepreneurs? Immigrant Self-Employment in Spain

2010 (ISBN 978908964243 1)

Marta Kindler

A Risky Business? Ukrainian Migrant Women in Warsaw's Domestic Work Sector

2011 (ISBN 9789089643278 )

IMISCOE Textbooks

Marco Martiniello, Jan Rath, Eds.

Selected Studies in International Migration and Immigrant Incorporation 2010 (ISBN 978908964 1601) 